\title{
Analysis of BIOMOVS II Uranium Mill Tailings Scenario 1.07 with the RESRAD Computer Code
}

by E.K. Gnanapragasam and C. Yu

Environmental Assessment Division,

Argonne National Laboratory, 9700 South Cass Avenue, Argonne, Illinois 60439

August 1997

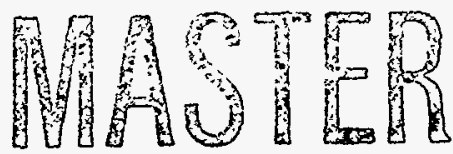

Work sponsored by United States Department of Energy, Office of Environment, Safety and Health 
This report is printed on recycled paper. 


\section{DISCLAMIER}

Portions of this document may be illegible in electronic image products. Images are produced from the best available original document. 


\section{CONTENTS}

ACKNOWLEDGMENT $\ldots \ldots \ldots \ldots \ldots \ldots \ldots \ldots \ldots \ldots \ldots \ldots \ldots \ldots \ldots$ vi

NOTATION $\ldots \ldots \ldots \ldots \ldots \ldots \ldots \ldots \ldots \ldots \ldots \ldots \ldots \ldots \ldots \ldots \ldots \ldots$ vii

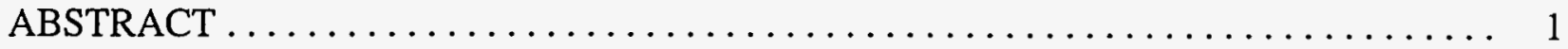

1 INTRODUCTION $\ldots \ldots \ldots \ldots \ldots \ldots \ldots \ldots \ldots \ldots \ldots \ldots \ldots \ldots \ldots \ldots$

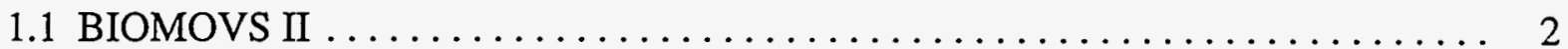

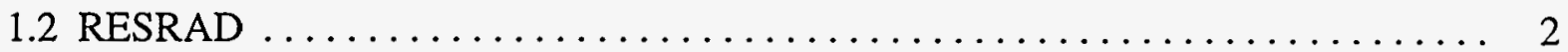

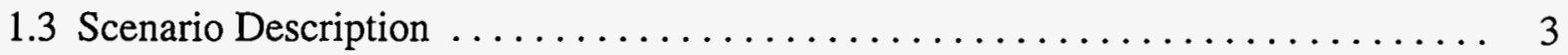

2 MODELING THE SCENARIO WITH RESRAD $\ldots \ldots \ldots \ldots \ldots \ldots \ldots \ldots \ldots$

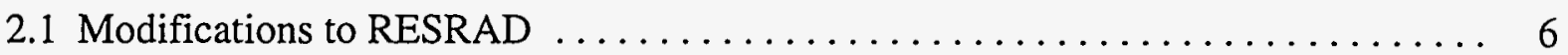

2.2 Simulation of the Scenario with $\operatorname{RESRAD} \ldots \ldots \ldots \ldots \ldots \ldots \ldots \ldots \ldots \ldots \ldots$

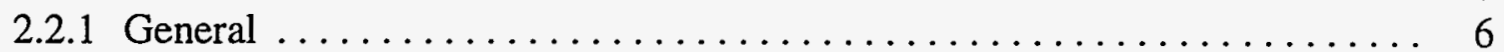

2.2.2 Atmospheric Source Term ...................... 8

2.2 .3 Groundwater Source Term ....................... 8

2.2.4 Off-Site Accumulation in Soil . . . . . . . . . . . . . . . . . 8

$3 \quad$ RESULTS $\ldots \ldots \ldots \ldots \ldots \ldots \ldots \ldots \ldots \ldots \ldots \ldots \ldots \ldots \ldots \ldots$

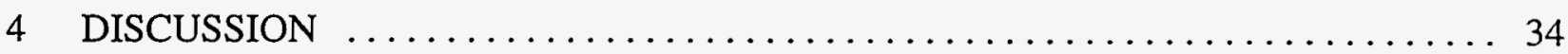

4.1 Deviations from Specified Scenario . . . . . . . . . . . . . . . . . 34

4.1.1 Off-Site Accumulation in Soil and Resuspension . . . . . . . . . . . . . 34

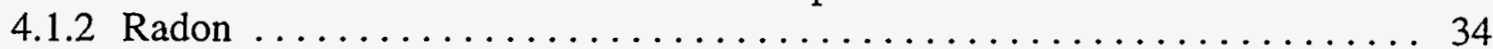

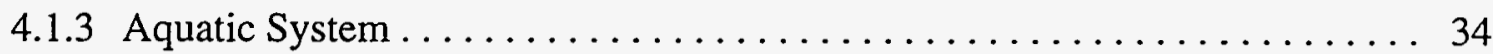

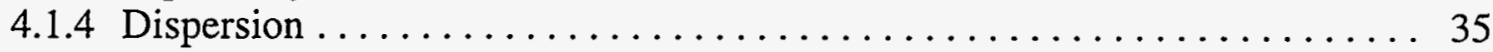

4.2 Suggestions to Extend Capability of RESRAD $\ldots \ldots \ldots \ldots \ldots \ldots \ldots$

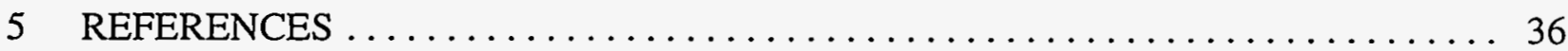

APPENDIX A: Scenario Description $\ldots \ldots \ldots \ldots \ldots \ldots \ldots \ldots \ldots \ldots \ldots \ldots \ldots$

APPENDIX B: Off-Site Accumulation Model $\ldots \ldots \ldots \ldots \ldots \ldots \ldots \ldots \ldots$

APPENDIX C: Tabular Results Requested in the Scenario $\ldots \ldots \ldots \ldots \ldots \ldots \ldots \ldots$

\section{FIGURES}

$1 \quad$ Layout of Scenario $\ldots \ldots \ldots \ldots \ldots \ldots \ldots \ldots \ldots \ldots \ldots \ldots \ldots \ldots \ldots$ 


\section{FIGURES (Cont.)}

2 Contribution of Pathways to Total Dose from Atmospheric Release $\ldots \ldots \ldots \ldots \ldots 11$

3 Contribution of Pathways to Total Dose from Groundwater Release $\ldots \ldots \ldots \ldots \ldots 12$

4 Total Radiological Dose from Radionuclides at Point of Exposure and Total Intake of Stable Elements for Groundwater Release $\ldots \ldots \ldots \ldots \ldots \ldots$

5 Radiological Dose from Radionuclides at Point of Exposure and Intake of Stable Elements Due to Ingestion of Drinking Water for Groundwater Release . . . . . 14

6 Radiological Dose from Radionuclides at Point of Exposure and Intake of Stable Elements Due to Ingestion of Lettuce for Groundwater Release $\ldots . . \ldots \ldots \ldots$

7 Radiological Dose from Radionuclides at Point of Exposure and Intake of Stable Elements Due to Ingestion of Beef for Groundwater Release $\ldots \ldots \ldots \ldots 16$

8 Radiological Dose from Radionuclides at Point of Exposure and Intake of Stable Elements Due to Ingestion of Fish for Groundwater Release . . . . . . . . . 17

9 Total Radiological Dose from Radionuclides at Point of Exposure and Total Intake of Stable Elements for Atmospheric Release $\ldots \ldots \ldots \ldots \ldots \ldots$

10 Radiological Dose Due to External Radiation from Radionuclides at Point of Exposure for Atmospheric Release . . . . . . . . . . . . . . . . . . . . 19

11 Radiological Dose from Radionuclides at Point of Exposure and Intake of Stable Elements Due to Inhalation for Atmospheric Release $\ldots \ldots \ldots \ldots \ldots$

12 Radiological Dose from Radionuclides at Point of Exposure and Intake of Stable Elements Due to Ingestion of Lettuce for Atmospheric Release $\ldots \ldots \ldots \ldots 21$

13 Radiological Dose from Radionuclides at Point of Exposure and Intake of Stable Elements Due to Ingestion of Beef for Atmospheric Release . . . . . . . . . 22

14 Concentrations of Radionuclides and Stable Elements in Well Water

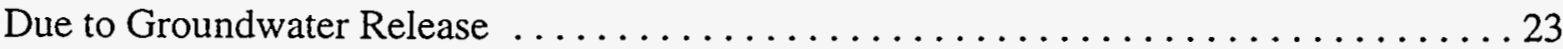

15 Concentrations of Radionuclides and Stable Elements in Lettuce Due to Groundwater Release . . . . . . . . . . . . . . . . . . . . . . . . . . . 24

16 Concentrations of Radionuclides and Stable Elements in Beef

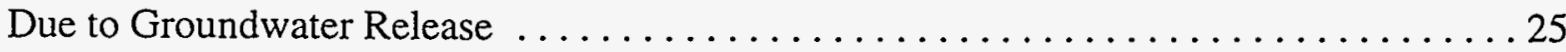

17 Concentrations of Radionuclides and Stable Elements in Fish Due to Groundwater Release ................................ 26 


\section{FIGURES (Cont.)}

18 Concentrations of Radionuclides and Stable Elements in Air

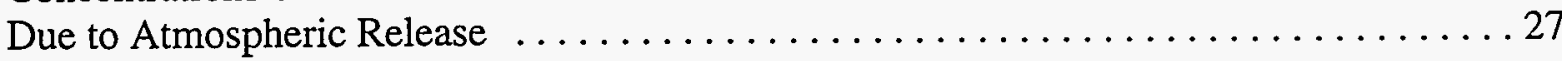

19 Concentrations of Radionuclides and Stable Elements in Off-Site Soil

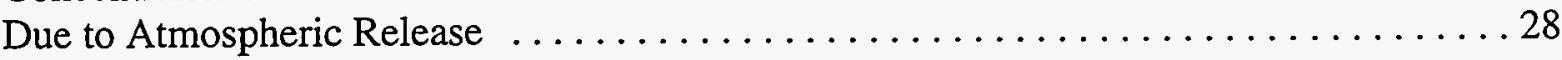

20 Concentrations of Radionuclides and Stable Elements in Lettuce

Due to Atmospheric Release . . . . . . . . . . . . . . . . . . . . . . . . . 29

21 Concentrations of Radionuclides and Stable Elements in Pasture

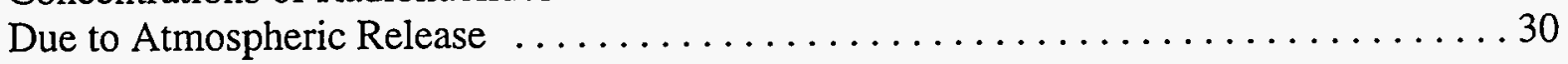

22 Concentrations of Radionuclides and Stable Elements in Beef

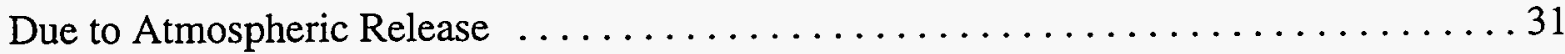

23 Excess Cancer Incidence Risk from Radionuclides at Point of Exposure

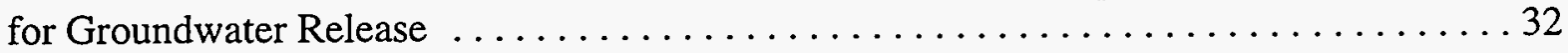

24 Excess Cancer Incidence Risk from Radionuclides at Point of Exposure and from Stable Elements for Atmospheric Release

\section{TABLE}

1 Input Values of Concentration and Distribution Coefficients of Contaminants in the Tailings 


\section{ACKNOWLEDGMENT}

The authors would like to thank Hal Peterson and Andy Wallo III of the U.S. Department of Energy, Office of Environment, Safety and Health, for sponsoring this study and reviewing this report. 


\section{NOTATION}

The following is a list of the acronyms, initialisms, and abbreviations (including units of measure) that appear in this document.

\section{ACRONYMS, INITIALISMS, AND ABBREVIATIONS}

$\begin{array}{ll}\text { ANL } & \text { Argonne National Laboratory } \\ \text { BIOMOVS II } & \text { Biospheric Model Validation Study: Phase II } \\ \text { DOE } & \text { U.S. Department of Energy } \\ \text { QA/QC } & \text { quality assurance/quality control } \\ \text { RESRAD } & \text { residual radioactive material guidelines (computer code) } \\ \text { SI } & \text { Système International } \\ \text { UMT } & \text { uranium mill tailings }\end{array}$

\section{ELEMENTS AND CHEMICALS}

As

$\mathrm{Cr}$

$\mathrm{Ni}$

$\mathrm{Pb}-210$

Po-210

Ra-226

$\mathrm{Rn}-222$

Th-230

U-234

U-238 arsenic

chromium

nickel

lead- 210

polonium-210

radium-226

radon-222

thorium-230

uranium-234

uranium-238

\section{UNITS OF MEASURE}

$\begin{array}{ll}\mathrm{Bq} & \text { becquerel(s) } \\ \mathrm{d} & \text { day(s) } \\ \mathrm{g} & \text { gram(s) } \\ \mathrm{ha} & \text { hectare(s) } \\ \mathrm{kg} & \text { kilogram(s) } \\ \mathrm{km} & \text { kilometer(s) } \\ \mathrm{L} & \text { liter(s) } \\ \mathrm{m} & \text { meter(s) } \\ \mathrm{m}^{2} & \text { square meter(s) } \\ \mathrm{m}^{3} & \text { cubic meter(s) } \\ \mathrm{mg} & \text { milligram(s) } \\ \mathrm{s} & \text { second(s) } \\ \mathrm{Sv} & \text { sievert(s) } \\ \mathrm{yr} & \text { year(s) }\end{array}$




\title{
ANALYSIS OF BIOMOVS II URANIUM MILL TAILINGS SCENARIO 1.07 WITH THE RESRAD COMPUTER CODE
}

by

E.K. Gnanapragasam and C. Yu

\begin{abstract}
The residual radioactive material guidelines (RESRAD) computer code developed at Argonne National Laboratory was selected for participation in the model intercomparison test scenario, version 1.07, conducted by the Uranium Mill Tailings Working Group in the second phase of the international Biospheric Model Validation Study. The RESRAD code was enhanced to provide an output attributing radiological dose to the nuclide at the point of exposure, in addition to the existing output attributing radiological dose to the nuclide in the contaminated zone. A conceptual model to account for off-site accumulation following atmospheric deposition was developed and showed the importance of considering this process for this off-site scenario. The RESRAD predictions for the atmospheric release compared well with most of the other models. The peak and steady-state doses and concentrations predicted by RESRAD for the groundwater release also agreed well with most of the other models participating in the study; however, the RESRAD plots showed a later breakthrough time and sharp changes compared with the plots of the predictions of other models. These differences were due to differences in the formulation for the retardation factor and to not considering the effects of longitudinal dispersion.
\end{abstract}

\section{INTRODUCTION}

As part of the quality assurance/quality control (QA/QC) program for the residual radioactive material guidelines (RESRAD) computer code, the U.S. Department of Energy (DOE) sponsored Argonne National Laboratory's (ANL's) participation in the international Biospheric Model Validation Study: Phase II (BIOMOVS II). The RESRAD code was one of the nine computer codes selected to participate in the BIOMOVS II Uranium Mill Tailings (UMT) Working Group's scenario version 1.07 computer model comparison study. The results of the model intercomparison 
study are described in the BIOMOVS II Technical Report 4 (BIOMOVS II 1995). The present report describes the modeling of the UMT scenario version 1.07 with RESRAD.

\subsection{BIOMOVS II}

The BIOMOVS $\Pi$ is an international cooperative study to test models designed to quantify the environmental transfer and bioaccumulation of radionuclides and other trace substances (BIOMOVS II 1995). In addition to (1) testing the accuracy of the predictions of environmental assessment models, (2) recommending priorities for future research to improve the accuracy of these predictions, and (3) explaining the differences observed in the predictions of different models participating in this study, BIOMOVS II also serves as a forum for interaction between modelers, aiding in the improvement of models. Various working groups exist within BIOMOVS II, each focusing on a specific issue of environmental modeling.

The UMT Working Group deals with the intercomparison of models that can be used to assess the long-term impacts of contaminants released from UMT. Scenario version 1.07 is the culmination of numerous iterations among the members of this working group in developing a hypothetical scenario, comparing predictions of the intermediate scenarios, and refining and clarifying the scenario to arrive at a reasonably well-defined scenario to serve as the basis for comparison of deterministic predictions of the models participating in the study.

The RESRAD code developers contributed to the development of the scenario, with predictions from RESRAD being submitted for version 1.05, which was the first intermediate scenario for which calculations were performed by the members of the working group. Additional features were incorporated into RESRAD to accommodate the requirements of the scenario as work progressed. This report describes the RESRAD predictions for scenario version 1.07 and the enhancements to the code that occurred during the participation in the study and suggests directions for further additions to the code on the basis of the comparison of RESRAD predictions with those of the other models.

\subsection{RESRAD}

RESRAD is a computer code developed at ANL for the DOE to calculate site-specific residual radioactive material guidelines and radiological dose and risk to an on-site individual at a radioactively contaminated site. The code is continuously improved and updated in response to suggestions from users and to incorporate new features that facilitate user interaction and increase the capabilities and flexibility of the code. RESRAD-CHEM, which was derived from RESRAD, is used to calculate risks from chemical contaminants. The RESRAD-CHEM database includes 151 chemicals. A recent improvement to RESRAD is the addition of a Latin hypercube-Monte Carlo 
preprocessor that allows statistical distributions to be specified in place of single values for input parameters. The code is being extended to include off-site modeling capability.

In the RESRAD code, a pathway analysis method is used in which a pathway sum is calculated to relate the radiological dose and risk to the concentration of a radionuclide in soil. The pathway sum is the total of the pathway factors for each of the applicable routes of exposure. The pathway factor accounts for radioactive decay and ingrowth, transport, transfer, (bio)accumulation, and radiological potency of the contaminant. RESRAD models the following exposure pathways:

- External radiation from the ground;

- Inhalation of contaminated dust, radon and radon progeny, and gaseous airborne radionuclides;

- Ingestion of plant food contaminated by root uptake, foliar deposition, and irrigation water;

- Ingestion of meat and milk contaminated by fodder, livestock water, and soil ingestion;

- Ingestion of fish and aquatic foods contaminated by lake water;

- Ingestion of water from a contaminated well or surface water source; and

- Ingestion of contaminated soil.

\subsection{SCENARIO DESCRIPTION}

The layout of the hypothetical UMT pile specified for this test scenario (version 1.07) is shown in Figure 1. A 1,000-m-long, 500-m-wide, 10-m-high UMT pile would release contaminants at a constant rate, both to the atmosphere and to the groundwater immediately below it, for a period of 1,000 years. The groundwater flows parallel to the length of the pile and would transport the contaminants to a 10-m-deep well and a river situated $1,000 \mathrm{~m}$ from the downgradient edge of the pile. The river flows through a lake in the test region. The test region is a 22.5-degree sector of a $10-\mathrm{km}$ radius centered on the pile. The test region consists of two zones: zone $A$ is within a radius of $5 \mathrm{~km}$ from the pile, and zone $\mathrm{B}$ is the rest of the test region. The receptors of interest for each zone are located at $2.5 \mathrm{~km}$ and at $7.5 \mathrm{~km}$ from the pile. Except for a 1-hectare plot of lettuce (located $2.5 \mathrm{~km}$ from the UMT pile) that is irrigated with well water, zone $\mathrm{A}$ is unirrigated pastureland. Zone $\mathrm{B}$ is evenly divided between unirrigated pasture and lettuce fields irrigated with lake water. The scenario stipulates that the leachate from the pile is diluted only by lateral dispersion and that 


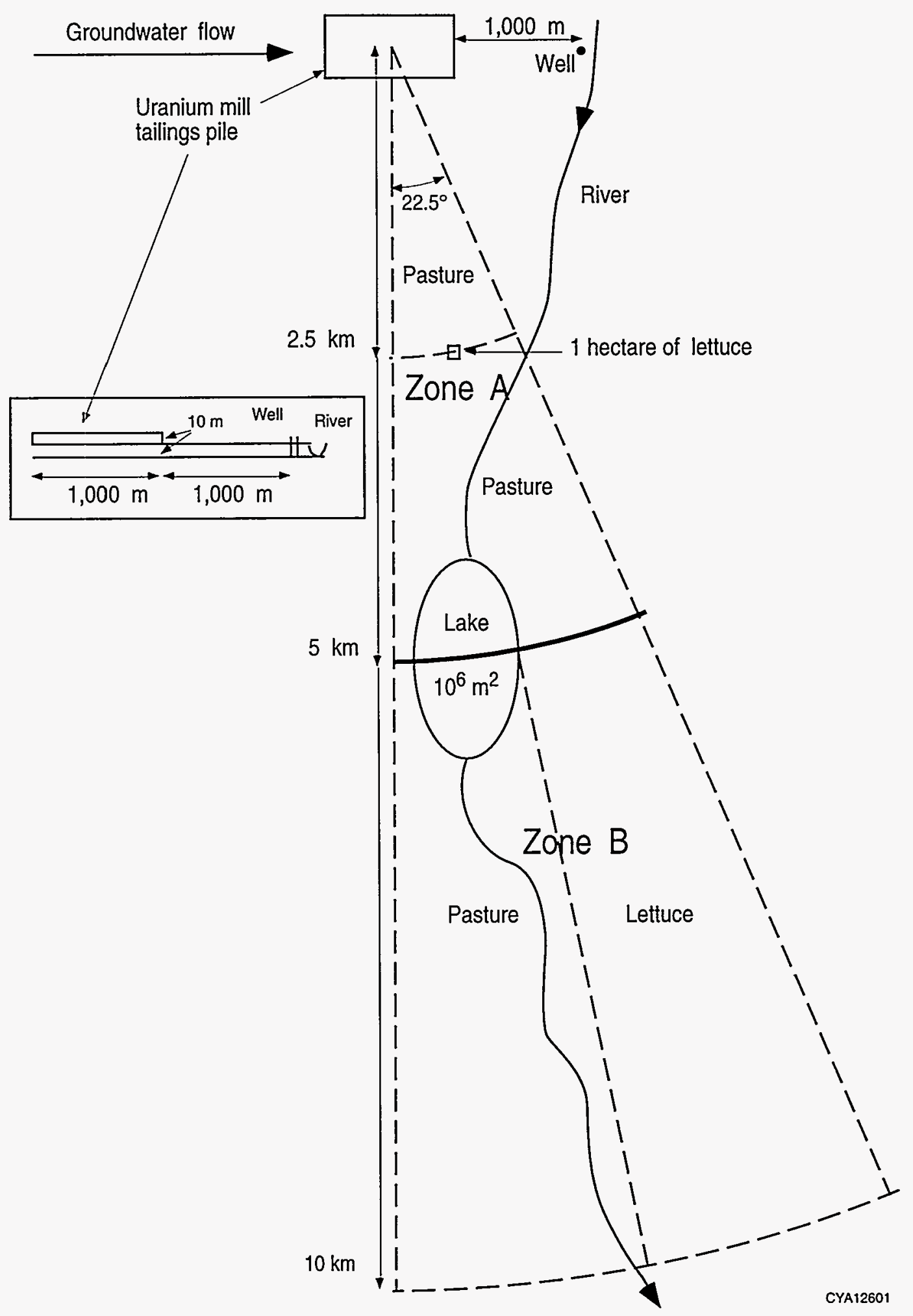

FIGURE 1 Layout of Scenario (Source: Modified from BIOMOVS II 1995) 
the river dilutes the contaminants by a factor of 10 . Livestock and humans in zone A obtain water from the well, and the lake is the source of water for the occupants of zone $B$. The atmospheric release is dispersed by wind that has a uniform wind rose.

The scenario required the prediction of the concentration of each contaminant in different media, the annual individual effective dose due to each radionuclide for each exposure pathway, the annual intake of each stable element for each exposure pathway, and the lifetime average risk of cancer incidence from all pathways. Separate predictions were required for the atmospheric source and the groundwater source.

The relevant sections of the scenario issued by the UMT Working Group are reproduced in Appendix A. 


\section{MODELING THE SCENARIO WITH RESRAD}

The application of RESRAD to UMT scenario version 1.07 is described in this chapter. Section 2.1 describes the modifications and additions made to RESRAD, release version 5.13, in order to model the scenario. The procedure used to model the scenario with RESRAD is described in Section 2.2.

\subsection{MODIFICATIONS TO RESRAD}

Excess cancer risk is reported in two forms in RESRAD. One of the forms allocates the risk to the radionuclides present at the point of exposure. The other form assigns the risk to the nuclide initially present in the contaminated zone. The dose is reported only in one format - as being due to the radionuclides initially present in the contaminated zone. The test scenario requires calculation of the dose from the nuclides present at the point of exposure. Therefore, a module was added to RESRAD to sum the dose due to each nuclide present at the point of exposure arising from all of the parent nuclides present in the contaminated zone.

The test scenario also specifies different interception factors for wet and dry deposition and also for different classes of vegetation (leafy vegetables and pasture). With RESRAD, the same interception factor is used for all classes of vegetation and types of deposition; therefore, the code was modified to accommodate six different interception factors. The following factors dealing with the interception pathway could not be changed by the user: deposition velocity, exposure period, annual yield, and weathering half-life. The values specified in the scenario were written into the modified RESRAD code.

\subsection{SIMULATION OF THE SCENARIO WITH RESRAD}

\subsubsection{General}

The test scenario uses SI (Système International) units of measure. Although the centimetergram-second system of units is used in RESRAD, RESRAD output in SI units can be obtained by specifying the soil concentrations in units of becquerels per gram, the dose factors in units of sieverts per becquerel, and the risk factors in units of risk per becquerel.

The scenario requires a constant release of the contaminants. The leaching model of RESRAD, in the absence of erosion of the contaminated zone, is a first-order rate equation. The amount of contaminants in the contaminated zone decreases exponentially under a first-order leach 
model. In such a situation, the release rate can be kept effectively constant by specifying low leach and mass loading rates coupled with a large inventory. Although this procedure minimizes the effect of leaching, the release rate decreases because of radioactive decay.

The scenario also requires that the releases stop after 1,000 years. The RESRAD code does not have a provision to truncate or modify the release after a specified time. Hence, two sets of runs, offset from each other by 1,000 years, were required. The difference between the two outputs gave the output for a truncated release. The offset run was required because radioactive decay, ingrowth, and leaching diminish the concentration of the contaminants to differing extents over the 1,000-year period. The values shown in Table 1 were used in combination with a solids emission rate of $0.01 \mathrm{~g} \mathrm{~s}^{-1}$ to obtain the specified atmospheric release rates.

The dilution factor of the on-site well is computed by RESRAD on the basis of the amount of water contributed to the well from infiltration through the contaminated zone and that from uncontaminated groundwater. A dilution factor of $1 / 12$ was computed for this scenario with RESRAD. Lateral dispersion, computed according to Section K.2 of Appendix K of the RESRAD manual (Yu et al. 1993), leads to a dilution factor of 0.84. Thus the RESRAD output had to be multiplied by $12 \times 0.84(=10.08)$ to meet the requirements of the scenario. Instead of multiplying each of the outputs, multiplying the contaminant concentration by that factor was easier. The combination of values used for the groundwater source is shown in Table 1.

TABLE 1 Input Values of Concentration and Distribution Coefficients of Contaminants in the Tailings

\begin{tabular}{|c|c|c|c|c|c|}
\hline \multirow[b]{3}{*}{ Contaminant } & \multirow[b]{3}{*}{$\begin{array}{c}{ }^{\mathrm{cz}} \mathrm{K}_{\mathrm{d}} \\
\left(\mathrm{m}^{3} \mathrm{~kg}^{-1}\right)\end{array}$} & \multicolumn{4}{|c|}{ Activity Concentration in Soil (Bq [or $\mathrm{mg} \mathrm{g}^{-1}$ ) } \\
\hline & & \multicolumn{2}{|c|}{ Atmospheric Source } & \multicolumn{2}{|c|}{ Groundwater Source } \\
\hline & & Initial & $\begin{array}{c}1,000-y r \\
\text { Offset }\end{array}$ & Initial & $\begin{array}{c}1,000-y r \\
\text { Offset }\end{array}$ \\
\hline $\mathrm{U}-238$ & 6.2 & 620 & 619.3 & 6,250 & 6,242 \\
\hline $\mathrm{U}-234$ & 6.2 & 620 & 619.3 & 6,250 & 6,242 \\
\hline Th-230 & 1,000 & 10,000 & 9,916 & 100,800 & 99,949 \\
\hline $\mathrm{Ra}-226$ & 50 & 10,000 & 9,983 & 100,800 & 100,629 \\
\hline $\mathrm{Pb}-210$ & 50 & 10,000 & 9,984 & 100,800 & 100,639 \\
\hline Po-210 & 50 & 10,000 & 9,983 & 100,800 & 100,629 \\
\hline As & 500 & 71.4 & 71.4 & 719.7 & 719.7 \\
\hline $\mathrm{Cr}$ & 1 & 7.14 & 7.089 & 719.7 & 71.46 \\
\hline $\mathrm{Ni}$ & 1 & 0.714 & 0.7089 & 719.7 & 7.146 \\
\hline
\end{tabular}




\subsubsection{Atmospheric Source Term}

Because RESRAD version 5.13 was designed for on-site cultivation on the land over the contaminated zone, several precautions were necessary to ensure that the desired off-site end points were obtained. A root depth of zero prevented root uptake from the contaminated zone. The fraction of water from the contaminated site was set to zero to suppress the contribution from the groundwater release. The external radiation, milk, fish, drinking water, soil ingestion, and radon pathways were suppressed. The dust mass loading rate was calculated by using Equation K.2 of Yu et al. (1993). The dust mass loadings for zones A and B were $2.56 \mathrm{E}-09$ and $4.64 \mathrm{E}-10 \mathrm{~g} \mathrm{~m}^{-3}$, respectively. An additional run was performed to account for root uptake following atmospheric deposition, as described in Section 2.2.4.

\subsubsection{Groundwater Source Term}

Setting the cover depth at $1 \mathrm{~m}$, which is greater than the root and mixing depths, ensured that direct root uptake from the contaminated zone and the dust subpathways were suppressed. The RESRAD code includes the assumption that all classes of vegetation are irrigated to the same extent with contaminated water. The scenario required that only lettuce be irrigated. Because no other means of contaminating the livestock fodder for the groundwater release existed, the livestock fodder intake was set to zero to suppress the irrigation of fodder. The RESRAD code also includes the assumption that any excess irrigation water passes through the contaminated zone (on-site cultivation). The infiltration through the contaminated zone was limited to the specified value of $0.1 \mathrm{~m} \mathrm{yr}^{-1}$ by the proper choice of evapotranspiration coefficient. Because RESRAD version 5.13 did not provide for off-site transport through the saturated zone, the methodology of Section K.2 of Appendix K of Yu et al. (1993) was used to simulate the saturated zone as an unsaturated zone of unit saturation ratio.

\subsubsection{Off-Site Accumulation in Soil}

In the test scenario, the two receptors are located at distances of $2.25 \mathrm{~km}$ and $7.25 \mathrm{~km}$ from the edge of the contamination. The agricultural and animal products consumed by the test individuals are also obtained from these same off-site locations. Atmospheric deposition and irrigation with contaminated water will lead to accumulation of contaminants at these off-site locations. The scenario stipulates that the off-site accumulation is removed at a half-life of 100 years. Version 5.13 of RESRAD cannot model this accumulation. Hence, a methodology to compute the off-site accumulation resulting from atmospheric deposition was developed (Appendix B). Because this methodology had not been implemented in the code at that time, the off-site soil content of the contaminants at different times was computed by using a spreadsheet. These off-site soil concentrations were then used in RESRAD runs to predict the external radiation dose and the contribution of root uptake from off-site accumulation due to atmospheric deposition. 


\section{RESULTS}

The tabular results requested by the scenario description are given in Appendix $C$. The maximum value in each column of each table is shown in bold type. The concentration, dose, and risk data for zone A are shown in Figures 2 through 24 (which are located at the end of Chapter 3 ). Data for zone B are not plotted because they are simply a rescaling of the zone A figures for all but the fish pathway and the sum over pathways. The fish from the lake are consumed by the receptors in both zones. The atmospheric release output for zone B is 0.18 times the output for zone A; the scaling factor for groundwater release is 0.12 .

The radiological dose from the atmospheric release received by the receptor in zone $A$ is shown in Figure 2. The dose from all active pathways increases over time because of the accumulation of contaminants in the off-site location. At the end of the 1,000-year release period, the contaminants that accumulate at the off-site location make up 30\% of the radiological dose. Thus the off-site accumulation process makes a significant contribution to the total dose and needs to be considered when the radiological consequences to an off-site receptor are being modeled. This rise over time is noticeable in Figure 12 (dose and intake from the ingestion of lettuce) for Ra-226, which has the highest soil-to-plant transfer factor in this scenario. In Figure 13 (dose and intake from the ingestion of beef), this effect is again most pronounced for $\mathrm{Ra}-226$; however, a noticeable rise occurs in the curves for $\mathrm{Cr}, \mathrm{Po}-210, \mathrm{Ni}, \mathrm{As}, \mathrm{U}-234$, and U-238. Root uptake following accumulation has a greater effect on the dose and intake from the ingestion of beef than on the dose and intake from the ingestion of lettuce. This greater effect occurs because the greater crop yield $\left(1.85 \mathrm{~kg} \mathrm{~m}^{-2}\right)$ and lower foliar interception factor (0.5) of pasture reduce the extent of contamination by foliar interception when compared with lettuce, which has a yield of $1.4 \mathrm{~kg} \mathrm{~m}^{-2}$ and a foliar interception factor of 0.7 .

The radiological dose to the receptor in zone $\mathrm{A}$ due to the groundwater release is shown in Figure 3. The dose contributions of all pathways mirror the shape of the concentration in groundwater shown in Figure 14 because off-site accumulation of contaminants from irrigation was not taken into account. The first increase in dose is due to the breakthrough of uranium at around 60 years, and the second is due to the breakthrough of radium, lead, and polonium at about 600 years. The dose decreases after 1,000 years and stabilizes at a steady value after 1,600 years, when the uranium and radium that were released in the 1,000-year release period pass the well. The thorium that was released into the aquifer does not break through in the 10,000-year prediction period, but the thorium (and its progeny) formed in transit from the uranium that was released accounts for the constant dose observed from 1,600 years to the end of the forecast horizon. The fluctuations about the steady value during this period, seen in Figure 3, are due to the use of two offset runs to model the truncated release. The two runs give nearly equal results, and rounding off these numbers to three or four significant figures causes these fluctuations when taking the difference 
between them. This observation illustrates the importance of providing the user with the option to input a step change in the release from the contaminated zone.

Agreement was good among the predictions of all of the models for the atmospheric release except in the case of off-site accumulation. The results for off-site accumulation varied because some models used a removal half-life that was different from the 100 -year value specified in the scenario description (BIOMOVS $I$ 1995). The RESRAD predictions for the atmospheric release compared well with the predictions of the other models in the study (Figures 2 through 18 of BIOMOVS II [1995]).

Considerable differences were found among the predictions of the nine models for the groundwater release. The peak and steady-state values predicted by RESRAD again agreed with the predictions of most of the other models; however, two important differences were seen in the RESRAD predictions. A later breakthrough was predicted by RESRAD, compared with the other models, due to the differences in the formulation for the retardation factor. Sharper changes in the concentration were also predicted by RESRAD, as opposed to the more gradual changes predicted by the other models, which accounted for longitudinal dispersion. 


\section{Radiological Dose}

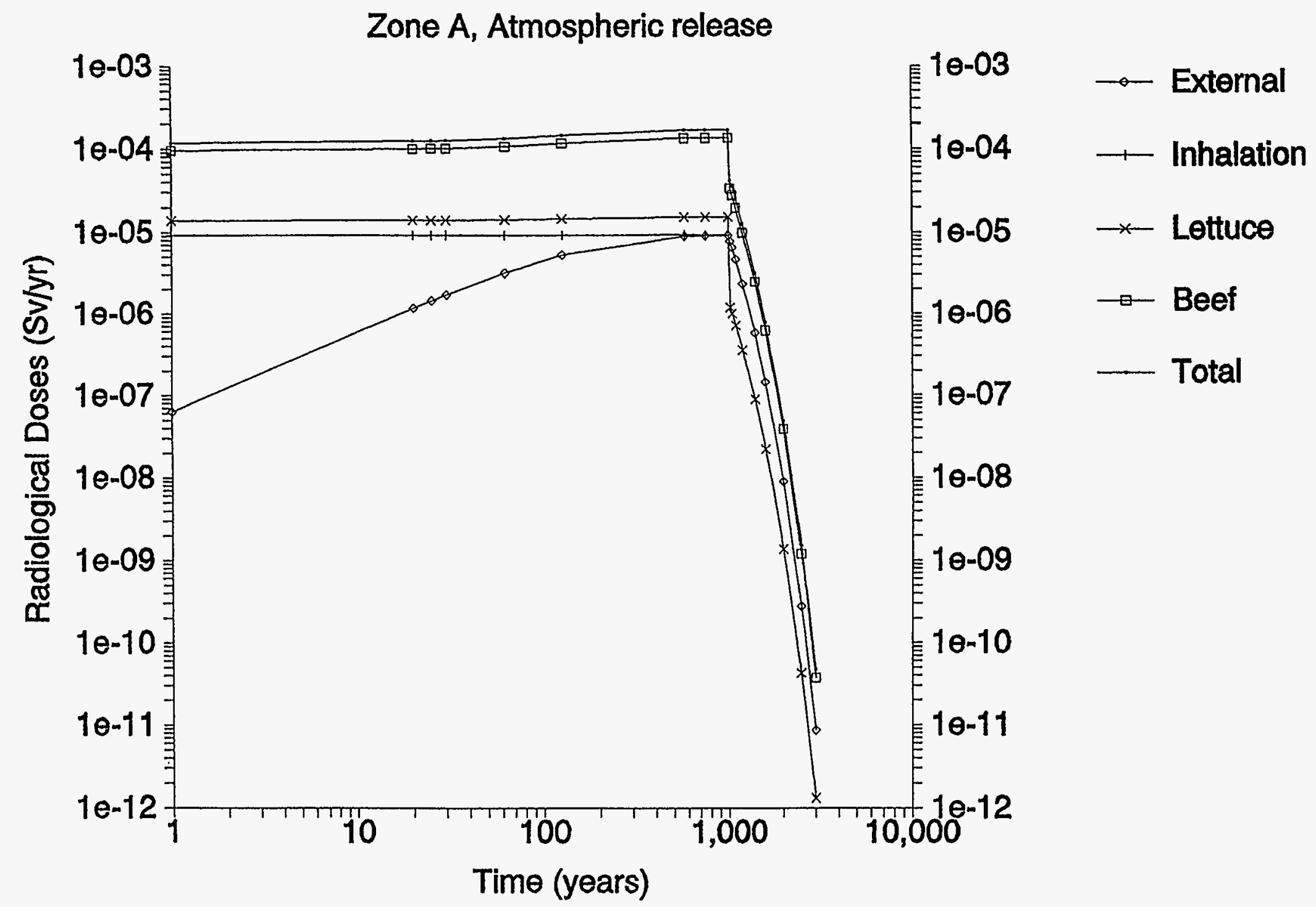

FIGURE 2 Contribution of Pathways to Total Dose from Atmospheric Release 


\section{Radiological Dose}

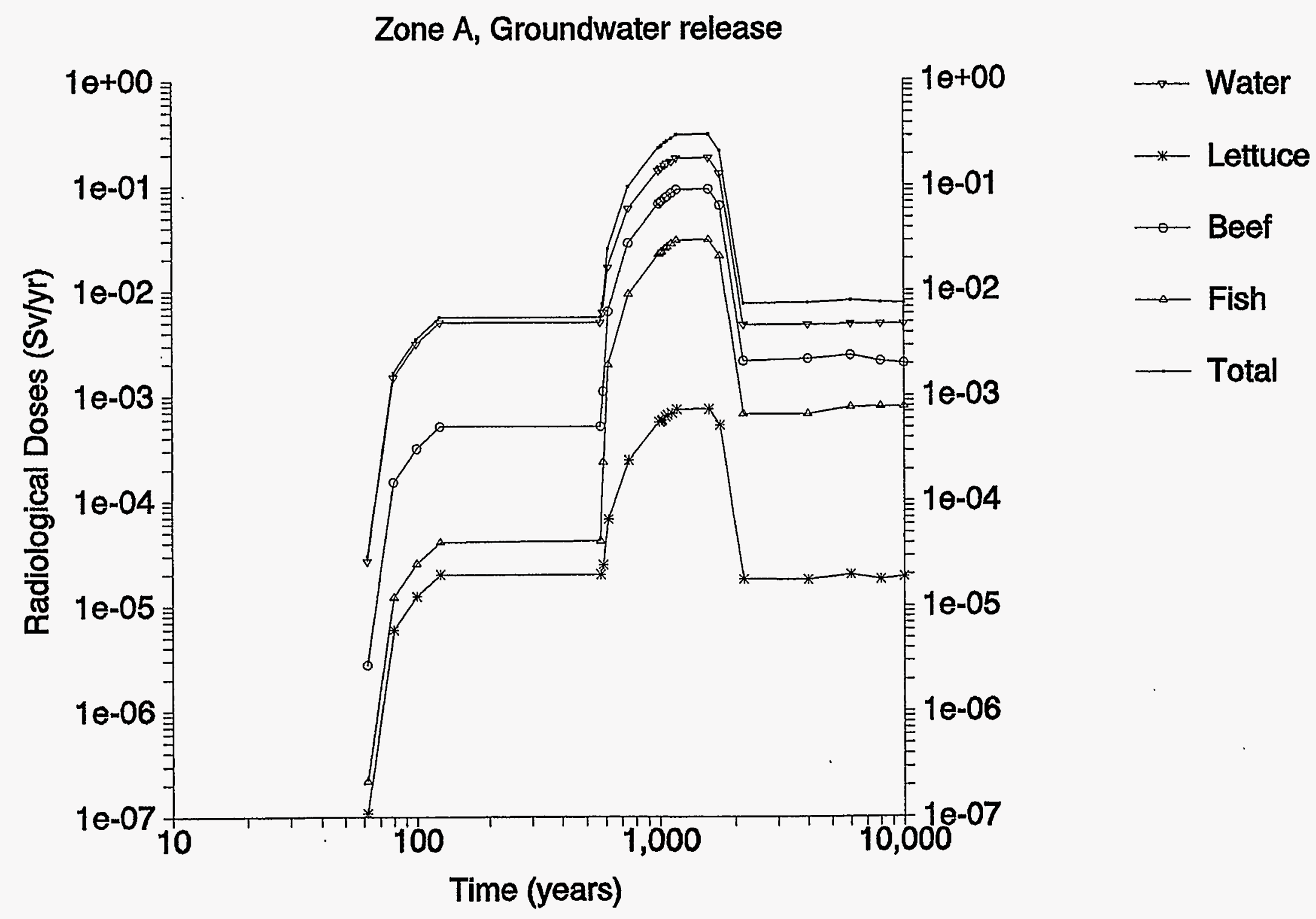

FIGURE 3 Contribution of Pathways to Total Dose from Groundwater Release 


\section{Total Dose and Intake}

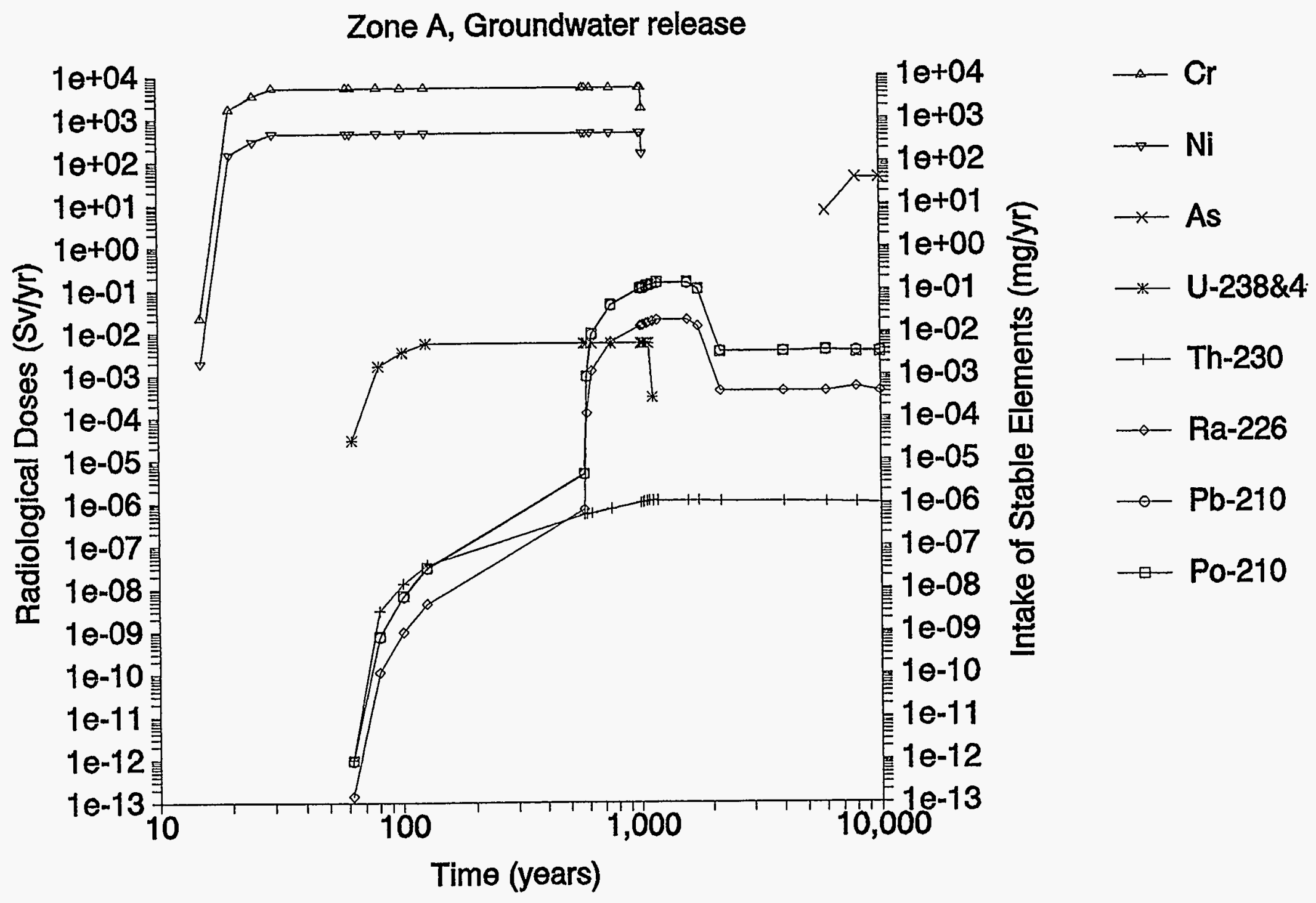

FIGURE 4 Total Radiological Dose from Radionuclides at Point of Exposure and Total Intake of Stable Elements for Ground water Release 


\section{Dose and Intake from Drinking Water}

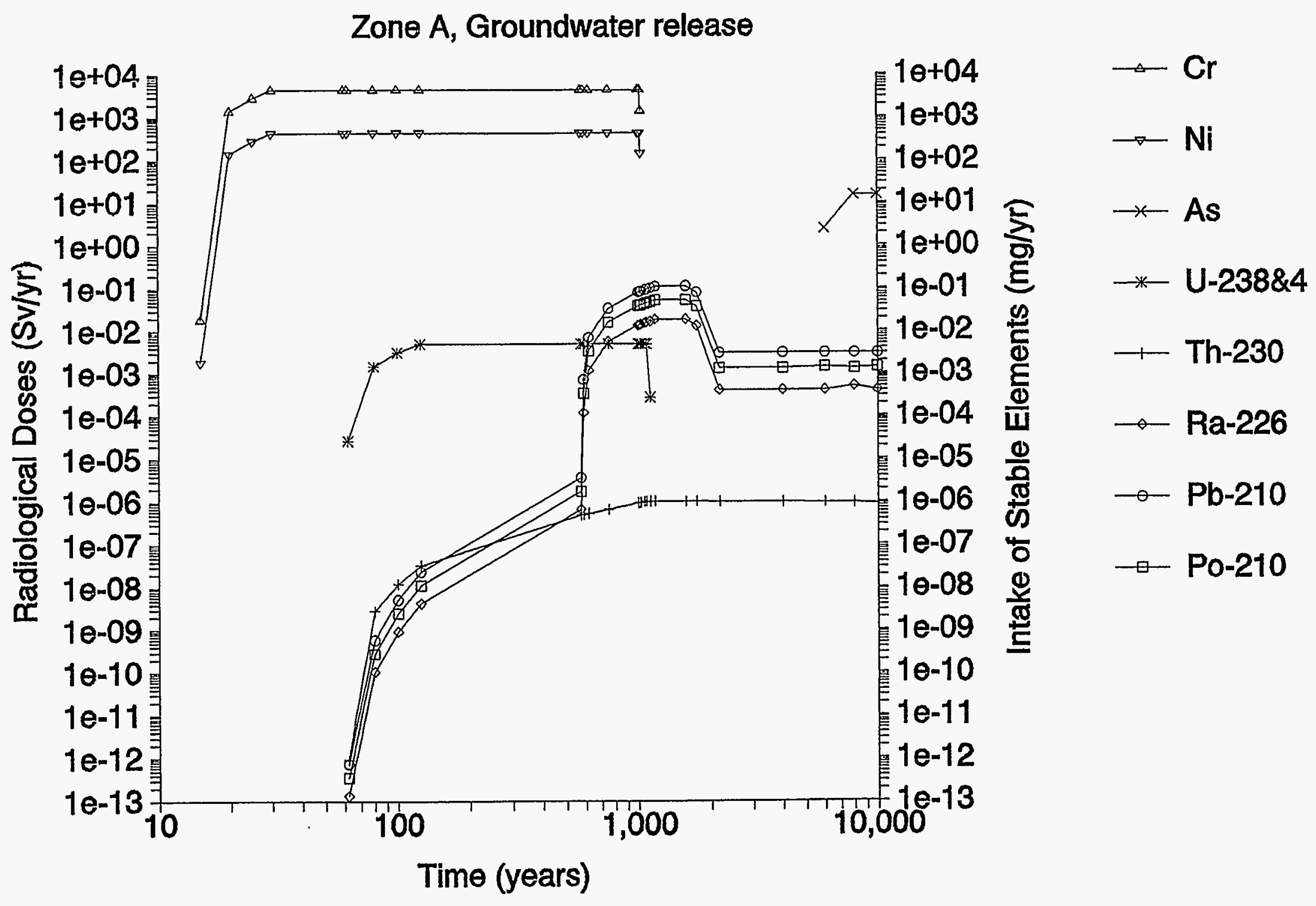

FIGURE 5 Radiological Dose from Radionuclides at Point of Exposure and Intake of Stable Elements Due to Ingestion of Drinking Water for Groundwater Release 


\section{Dose and Intake from Lettuce}

Zone A, Groundwater release

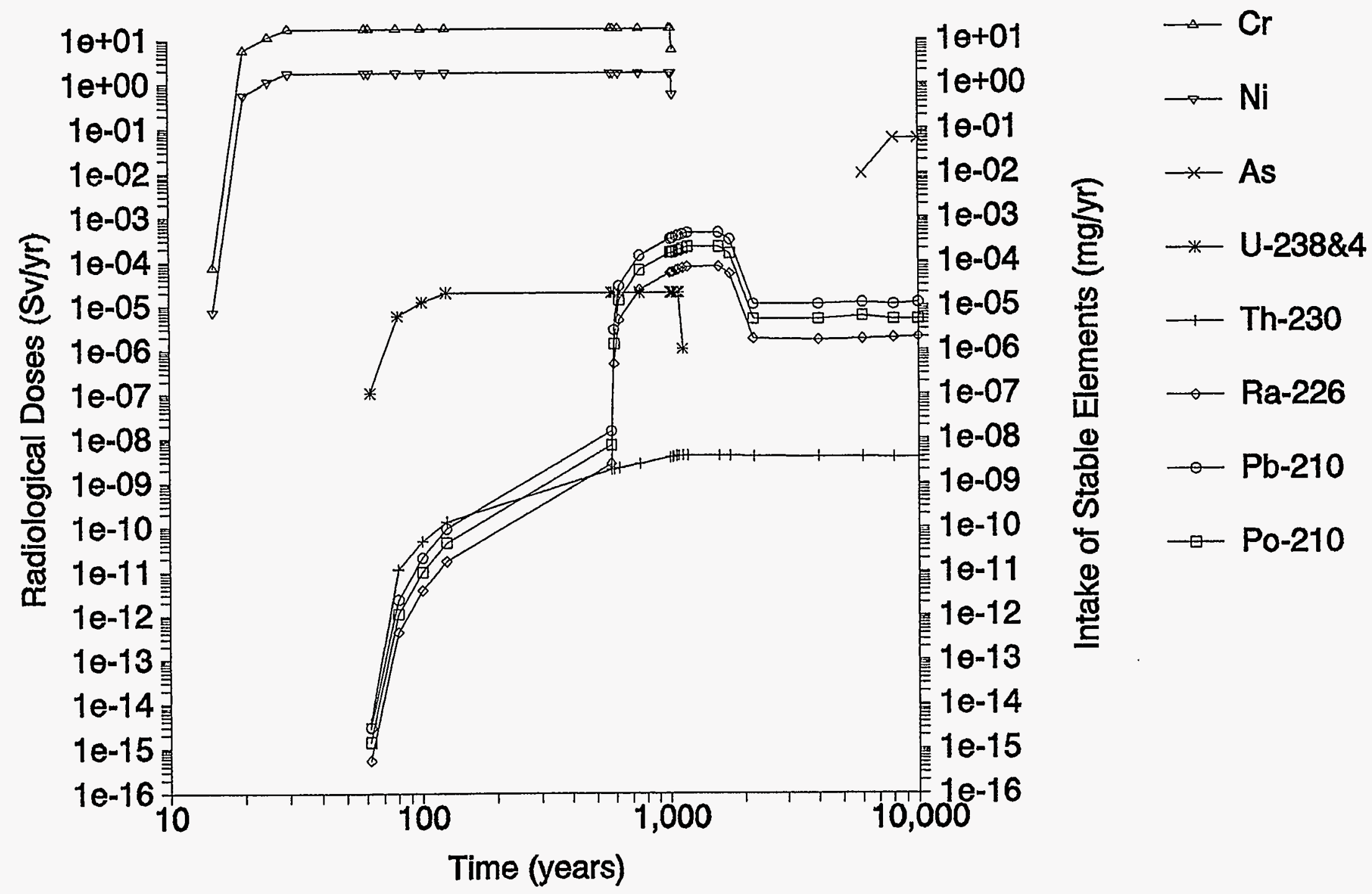

FIGURE 6 Radiological Dose from Radionuclides at Point of Exposure and Intake of Stable Elements Due to Ingestion of Lettuce for Groundwater Release 


\section{Dose and Intake from Beef}

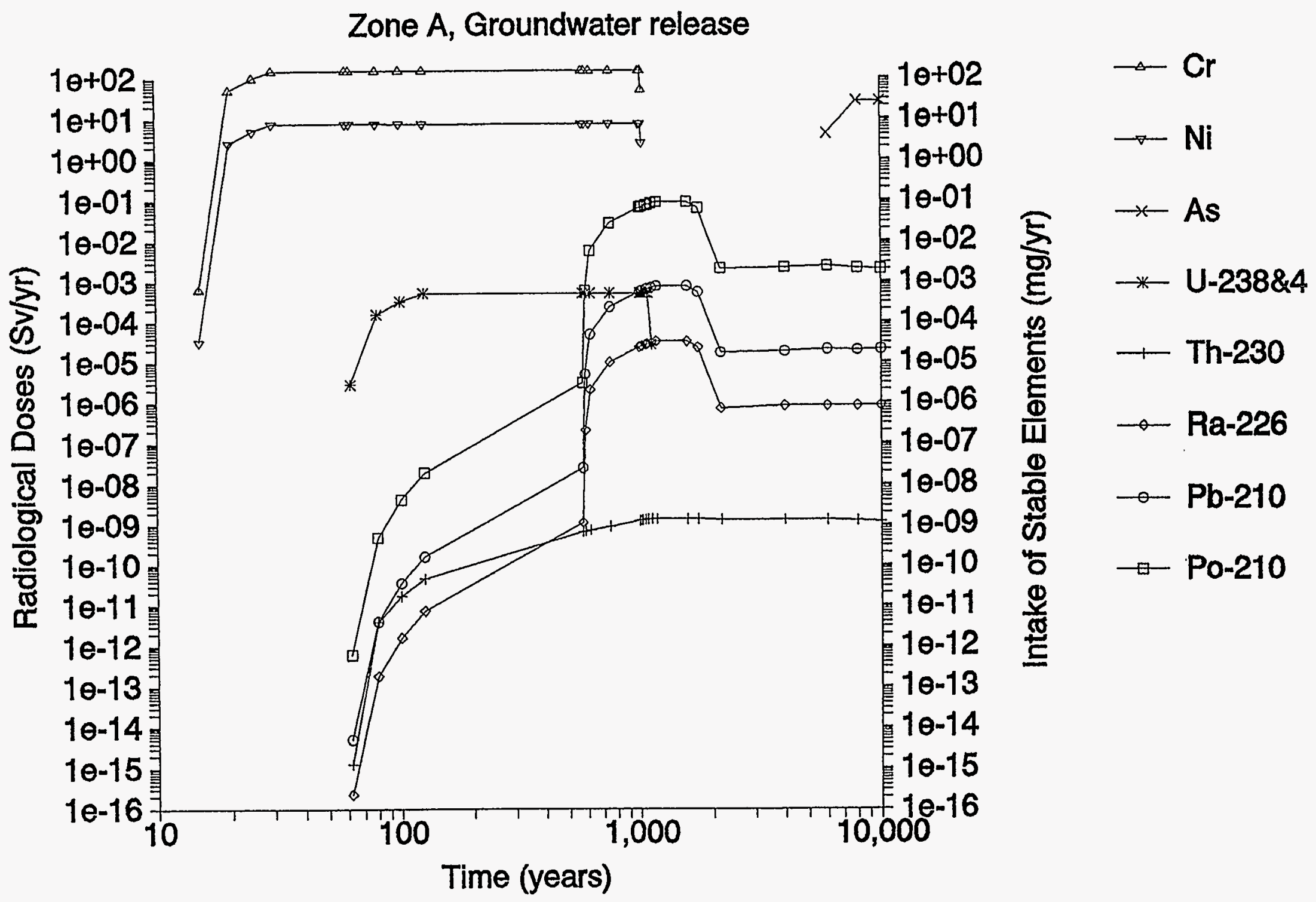

FIGURE 7 Radiological Dose from Radionuclides at Point of Exposure and Intake of Stable Elements Due to Ingestion of Beef for Groundwater Release 
Dose and Intake from Fish

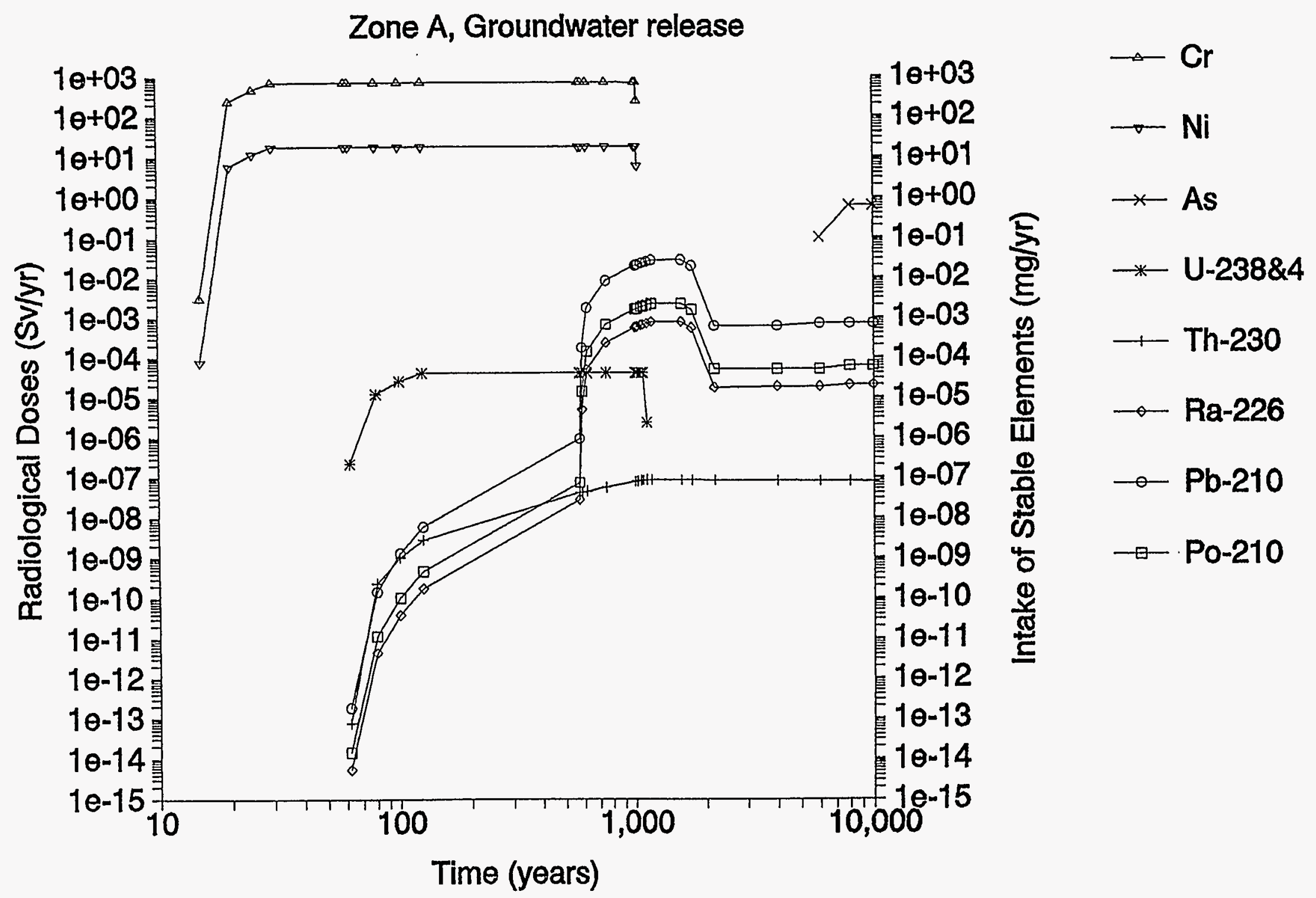

FIGURE 8 Radiological Dose from Radionuclides at Point of Exposure and Intake of Stable Elements Due to Ingestion of Fish for Ground water Release 


\section{Total Dose and Intake}

\section{Zone A, Atmospheric release}

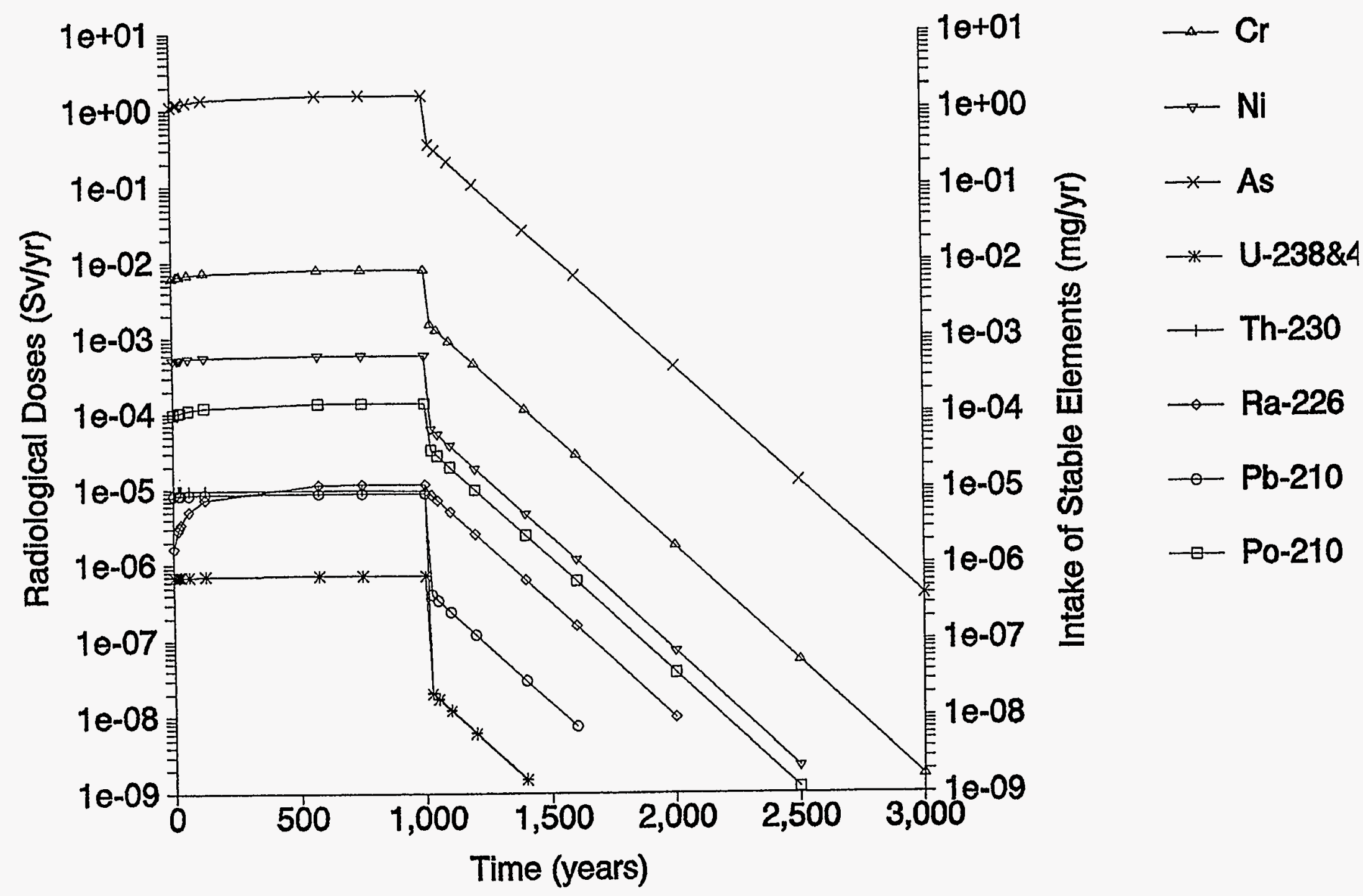

FIGURE 9 Total Radiological Dose from Radionuclides at Point of Exposure and Total Intake of Stable Elements for Atmospheric Release 


\section{Dose due to External radiation}

\section{Zone A, Atmospheric release}

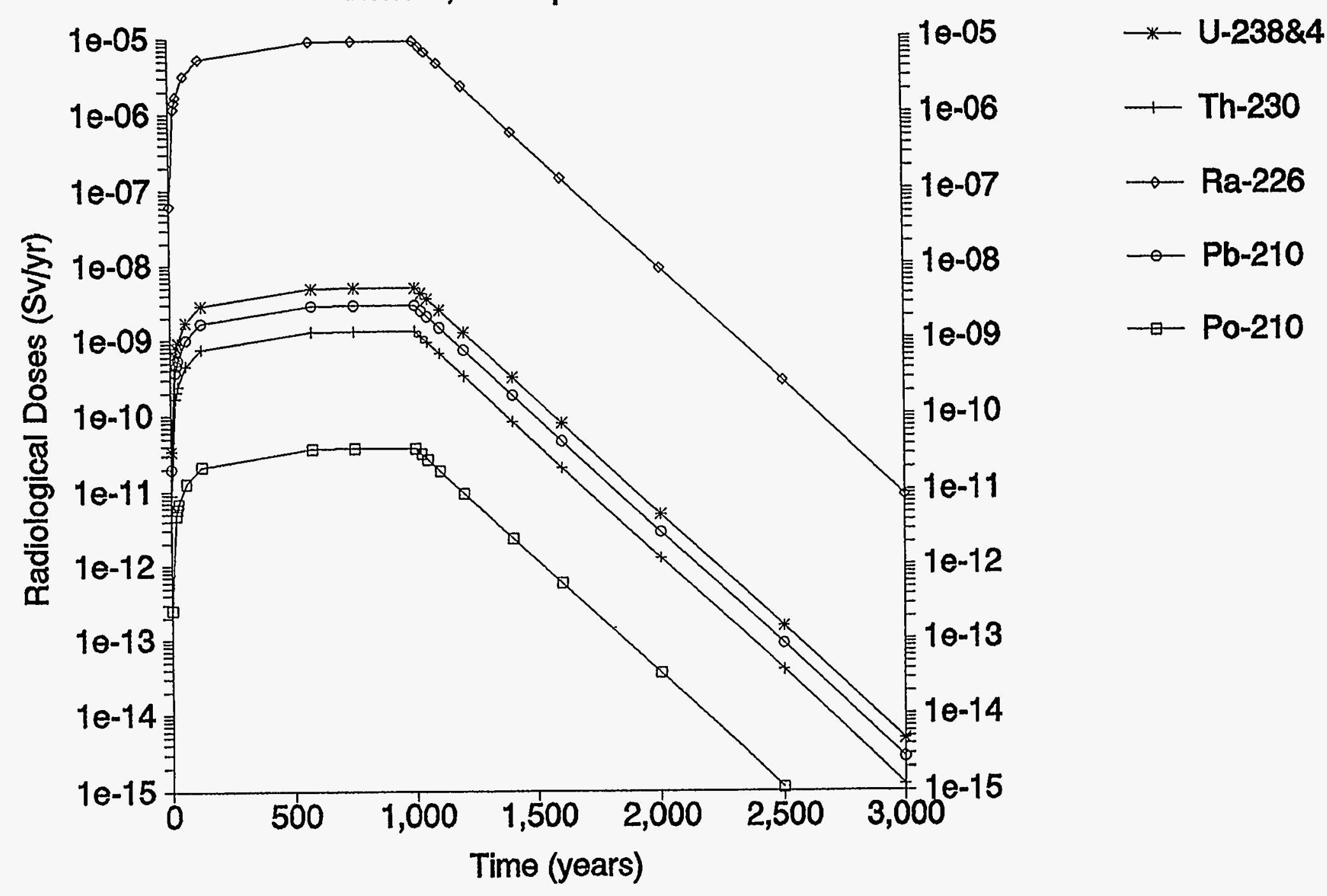




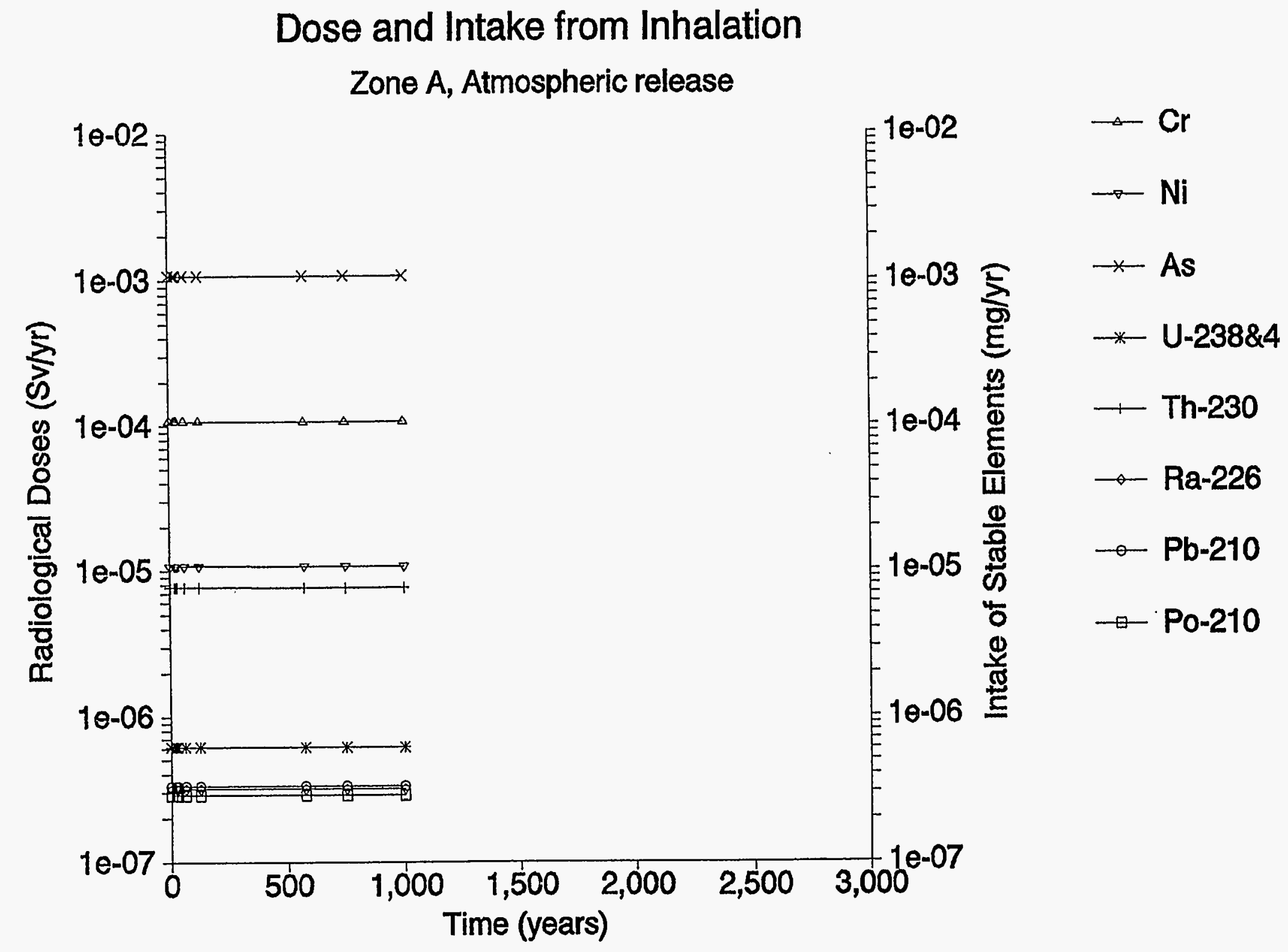

FIGURE 11 Radiological Dose from Radionuclides at Point of Exposure and Intake of Stable Elements Due to Inhalation for Atmospheric Release 


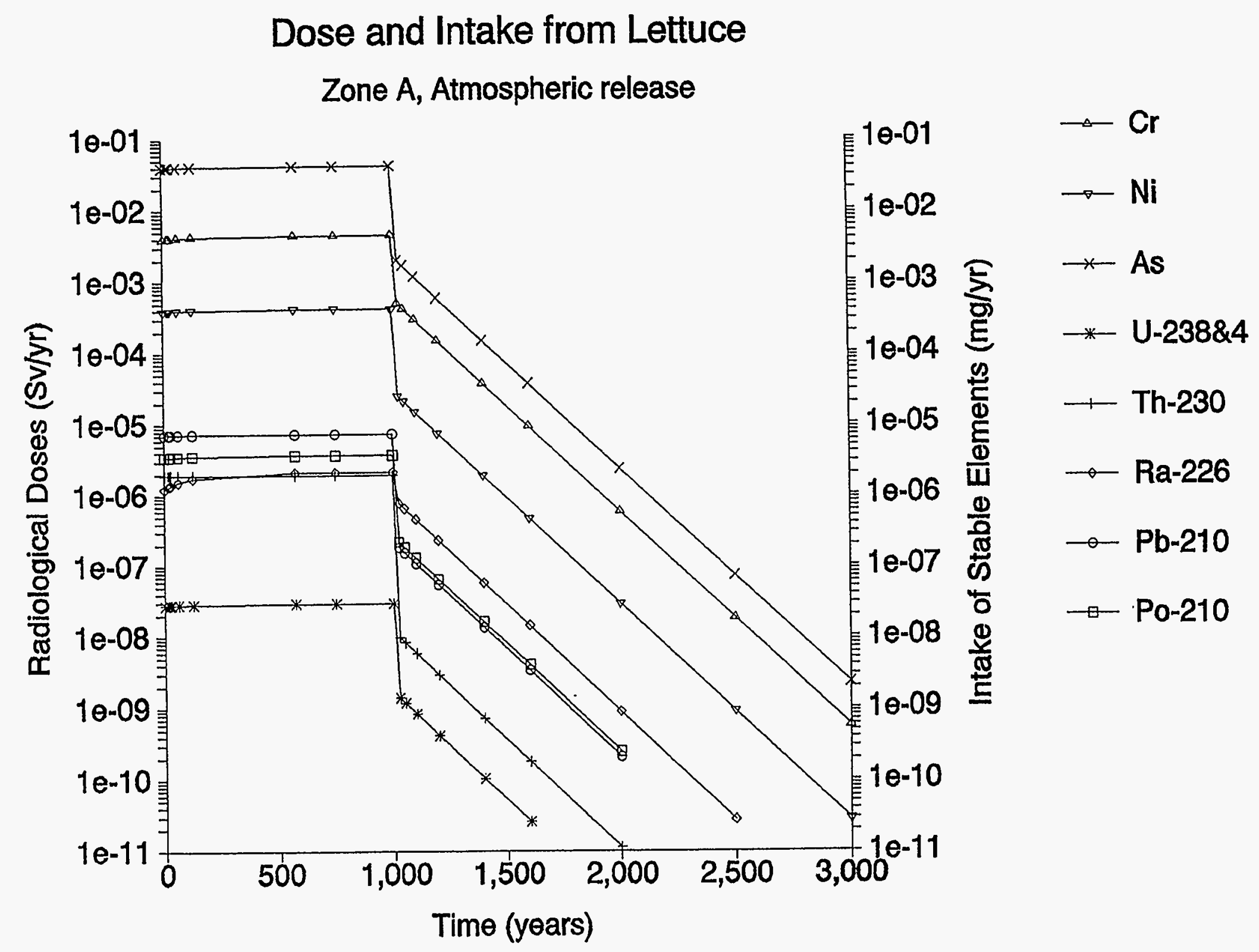

FIGURE 12 Radiological Dose from Radionuclides at Point of Exposure and Intake of Stable Elements Due to Ingestion of Lettuce for Atmospheric Release 


\section{Dose and Intake from Beef}

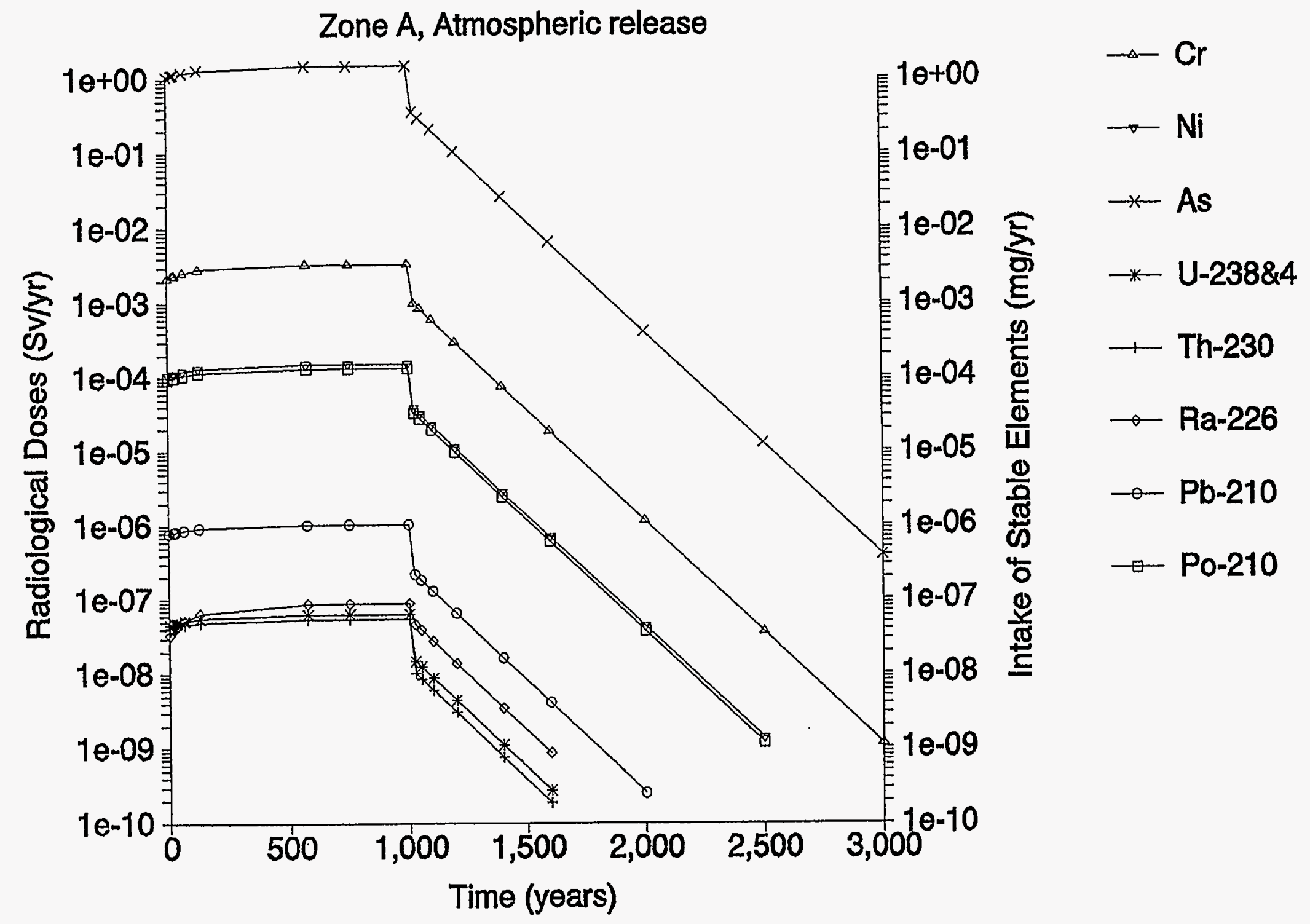

FIGURE 13 Radiological Dose from Radionuclides at Point of Exposure and Intake of Stable Elements Due to Ingestion of Beef for Atmospheric Release 


\section{Concentration of contaminants in Well water}

Zone A, Groundwater release

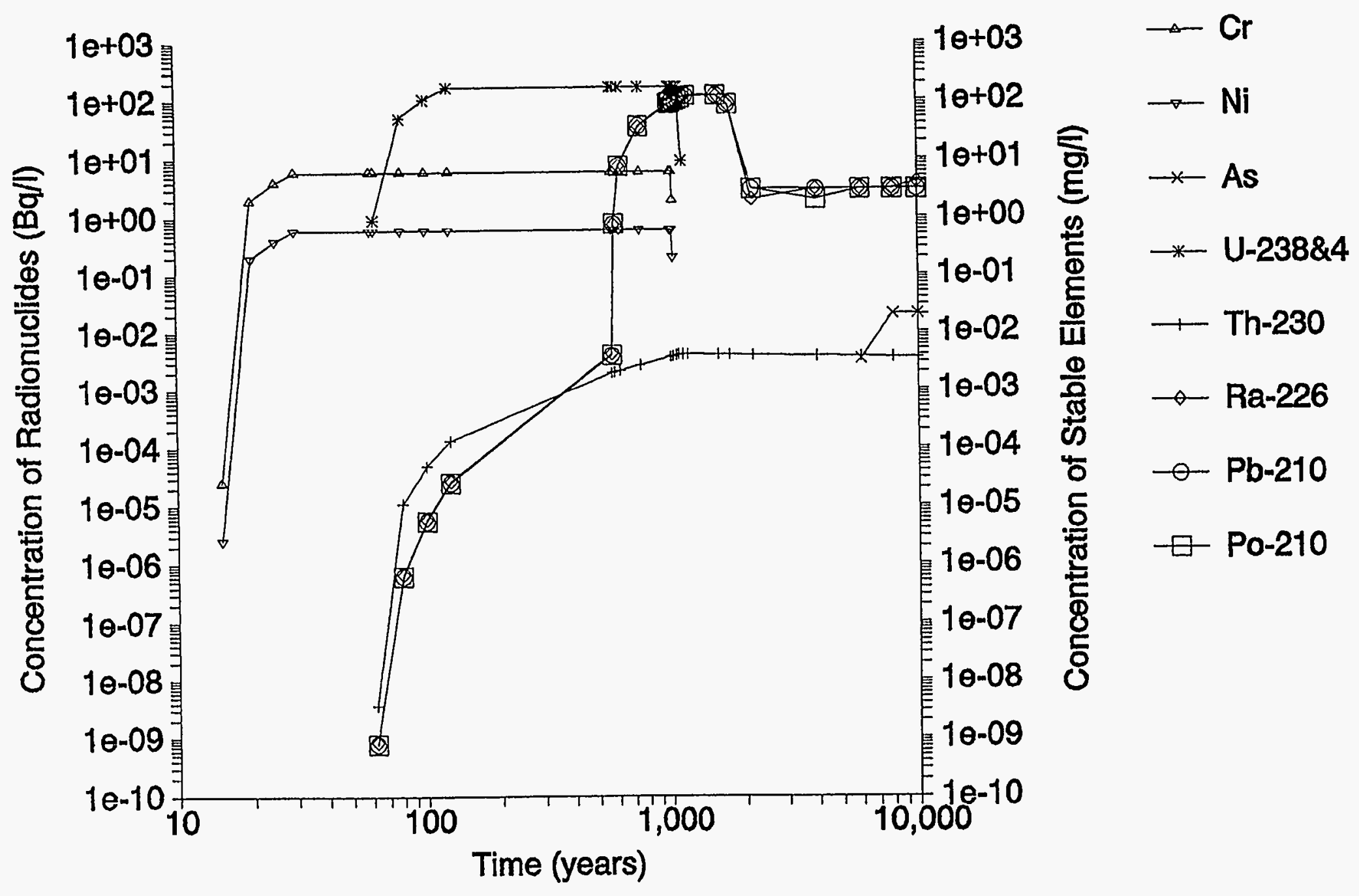


Concentration of contaminants in Lettuce

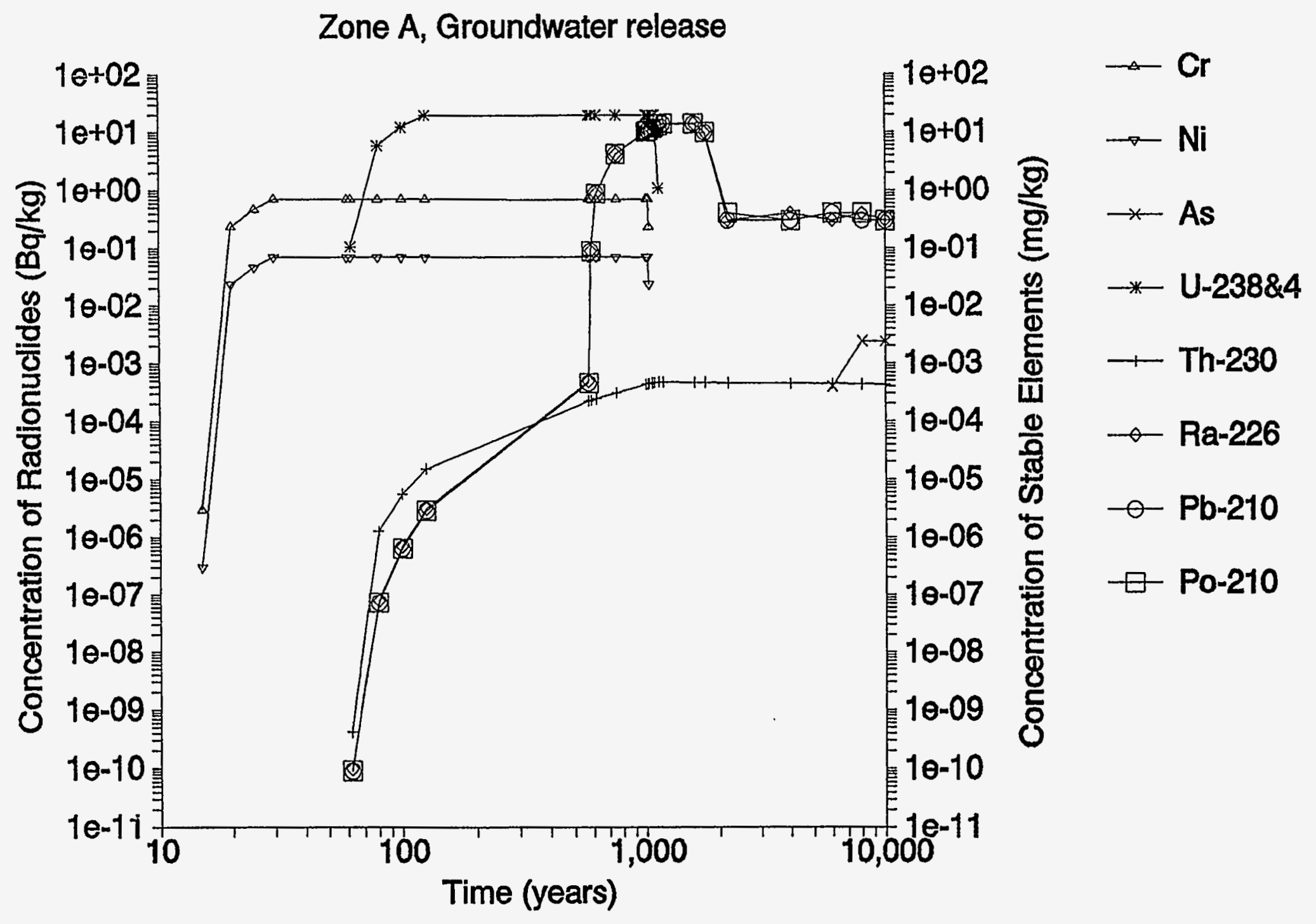




\section{Concentration of contaminants in Beef}

Zone A, Groundwater release

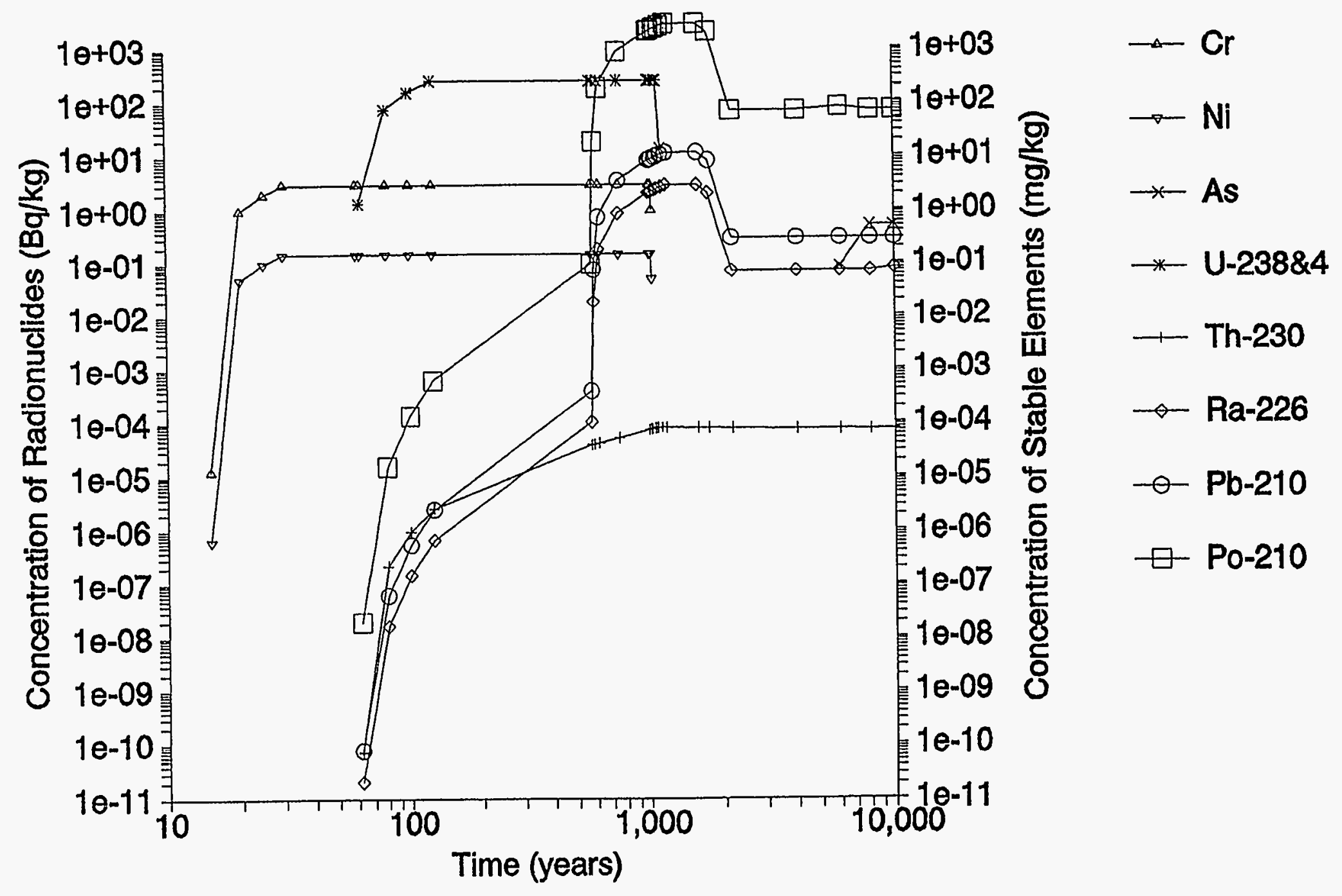




\section{Concentration of contaminants in Fish}

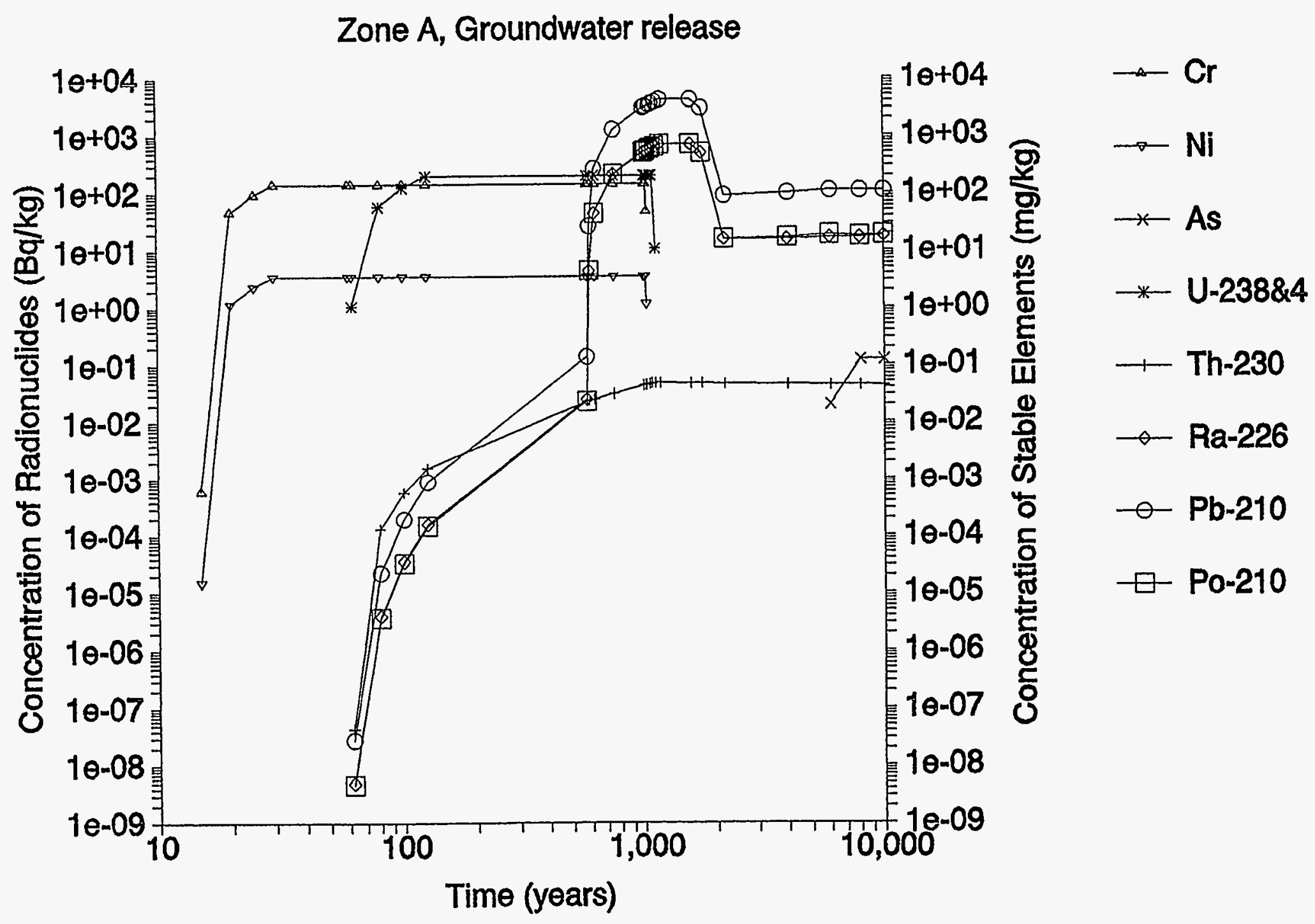


Concentration of contaminants in Atmosphere

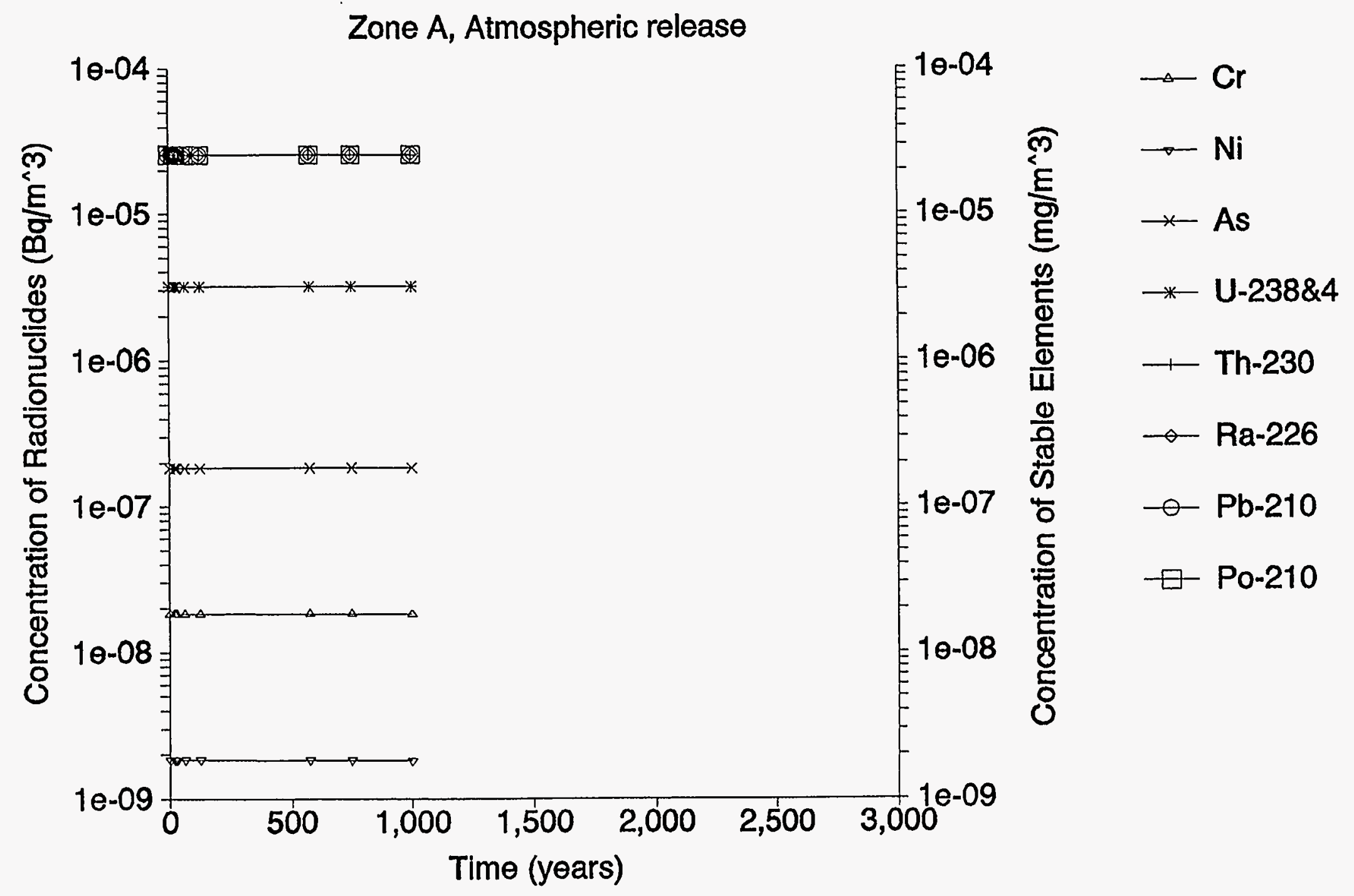




\section{Concentration of contaminants in Off-site Soil}

Zone A, Atmospheric release

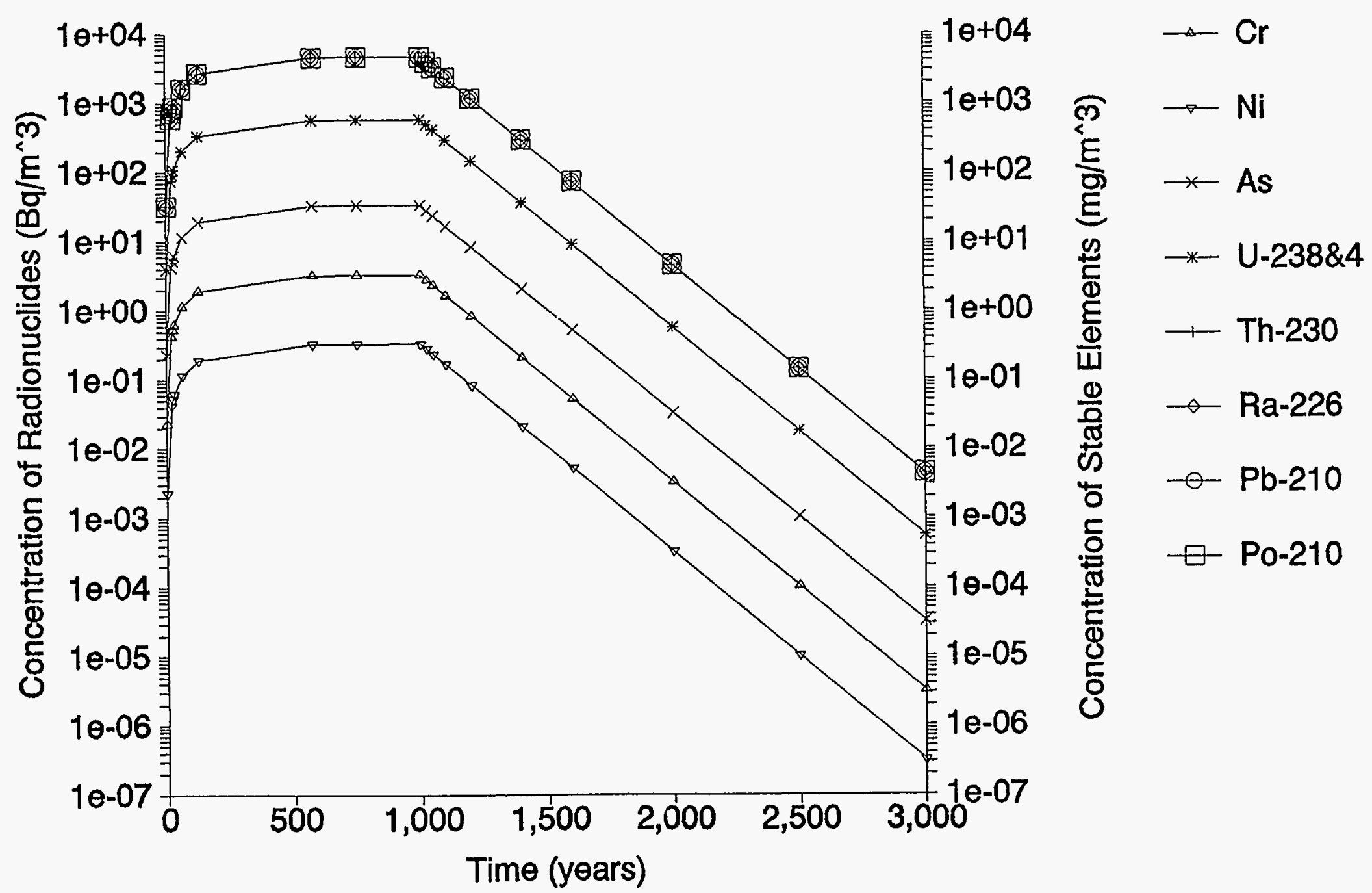

FIGURE 19 Concentrations of Radionuclides and Stable Elements in Off-Site Soil Due to Atmospheric Release 


\section{Concentration of contaminants in Lettuce}

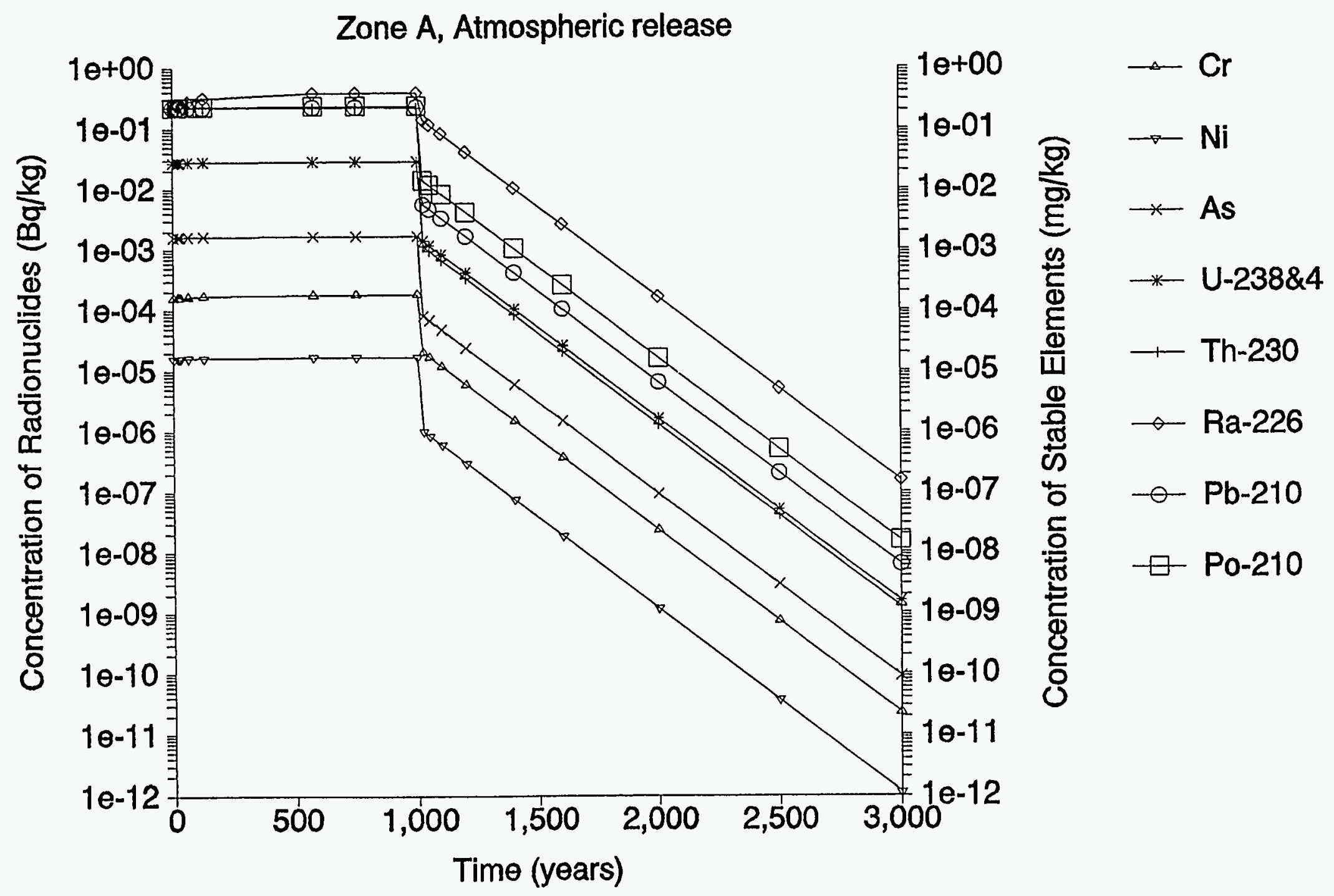

FIGURE 20 Concentrations of Radionuclides and Stable Elements in Lettuce Due to Atmospheric Release 


\section{Concentration of contaminants in Pasture}

Zone A, Atmospheric release

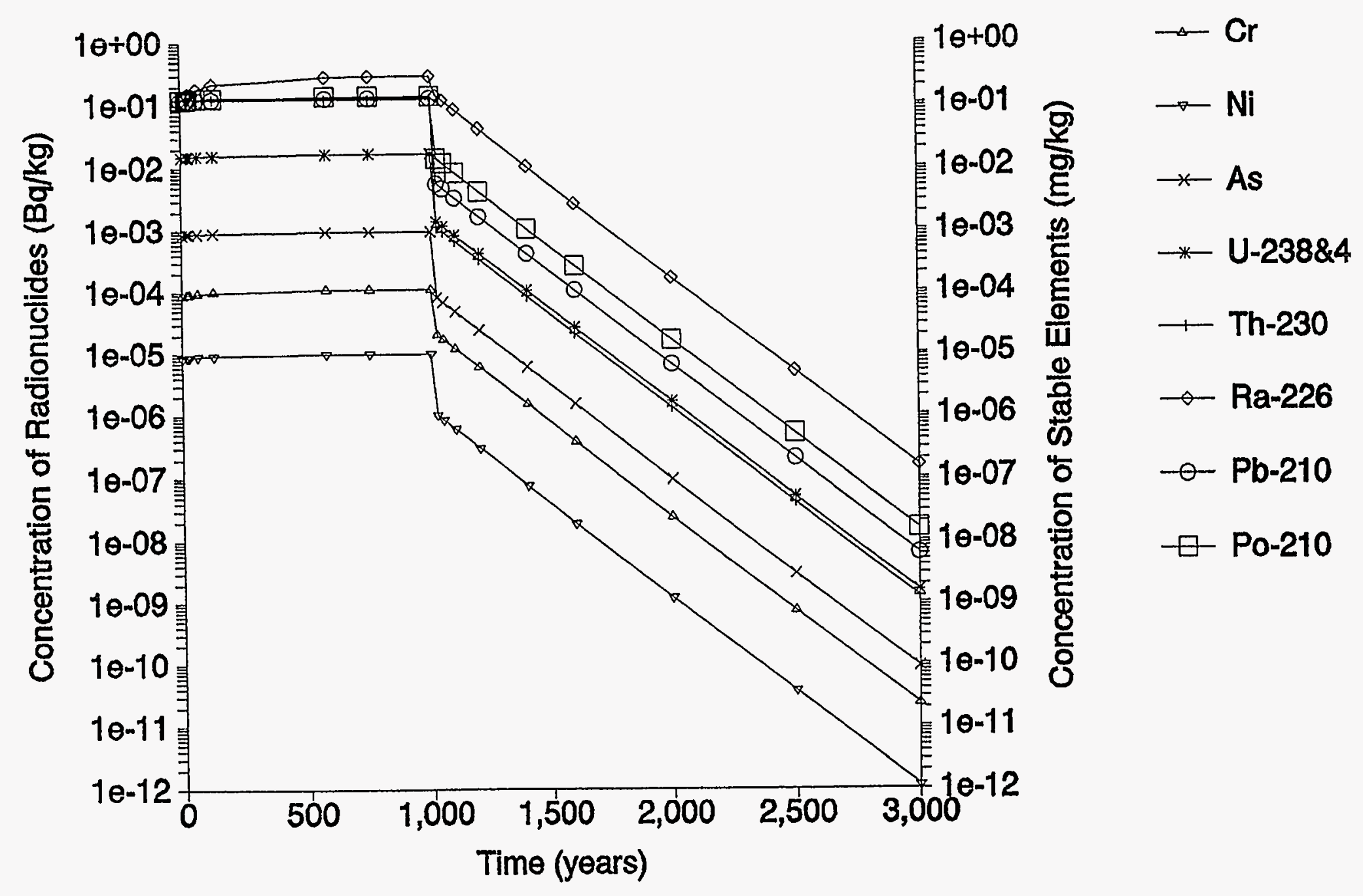

FIGURE 21 Concentrations of Radionuclides and Stable Elements in Pasture Due to Atmospheric Release 


\section{Concentration of Contaminants in Beef}

Zone As Atmospheric release

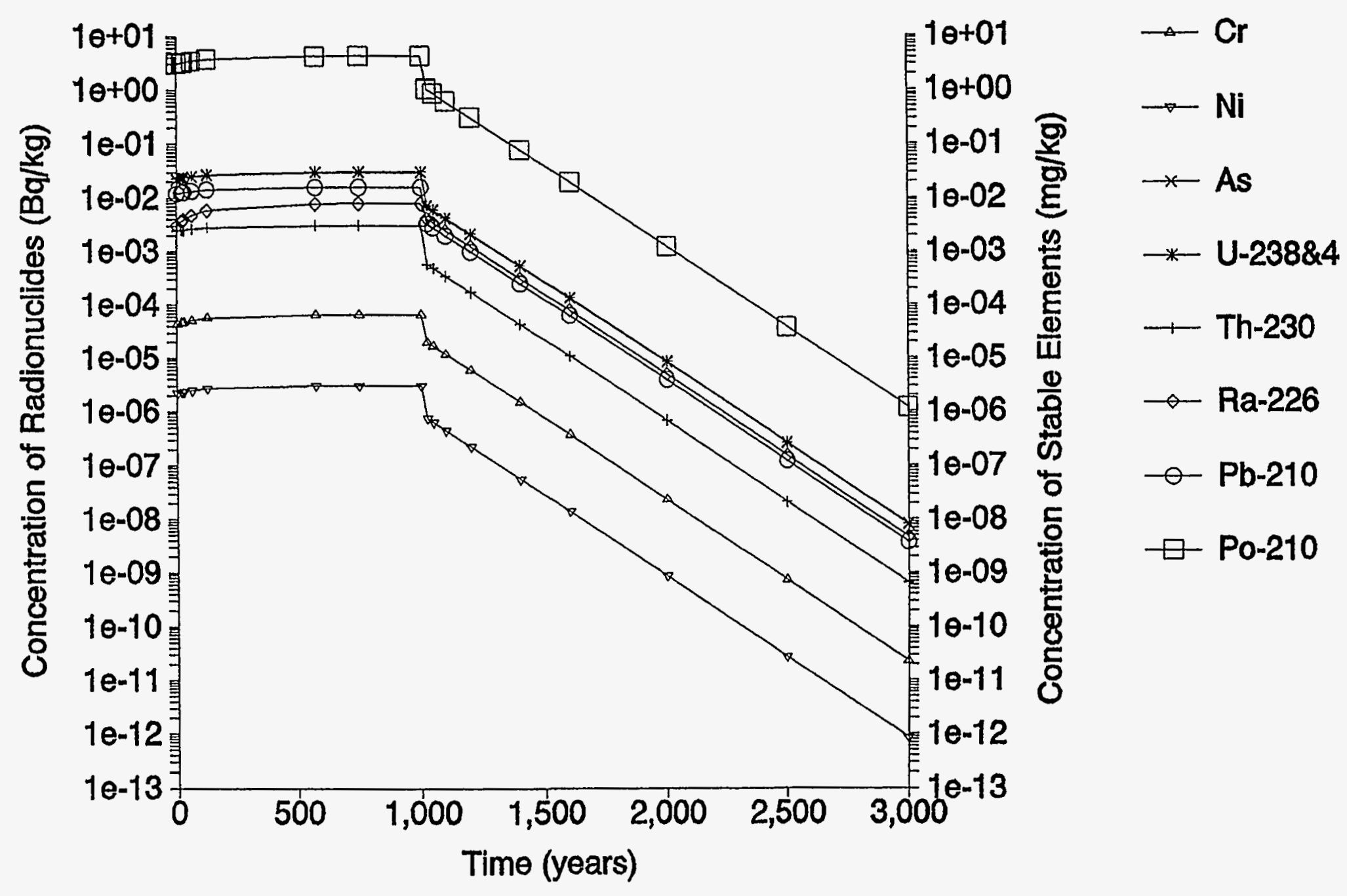




\section{Lifetime Excess Cancer incidence Risk}

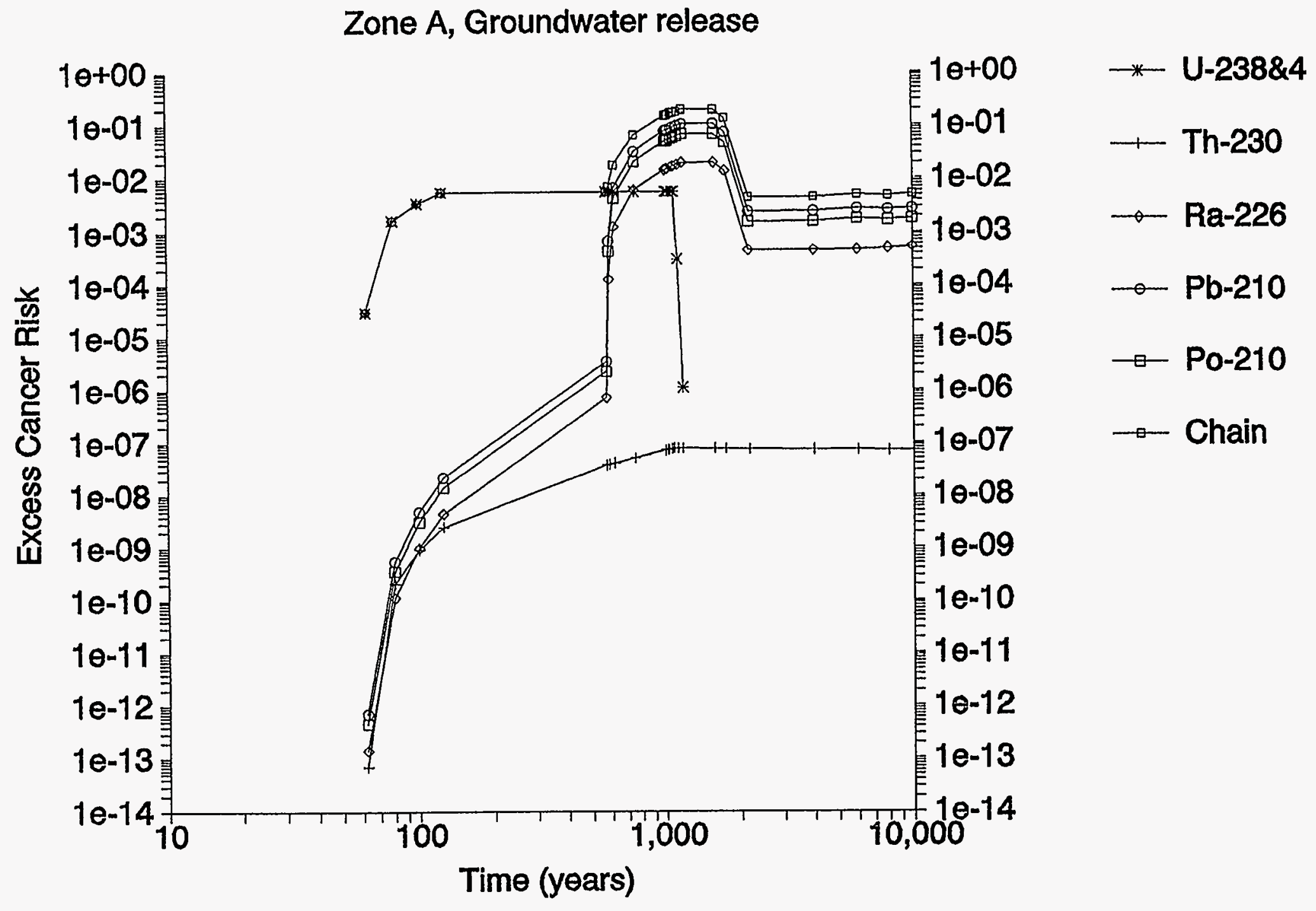




\section{Lifetime Excess Cancer incidence Risk}

Zone A, Atmospheric release

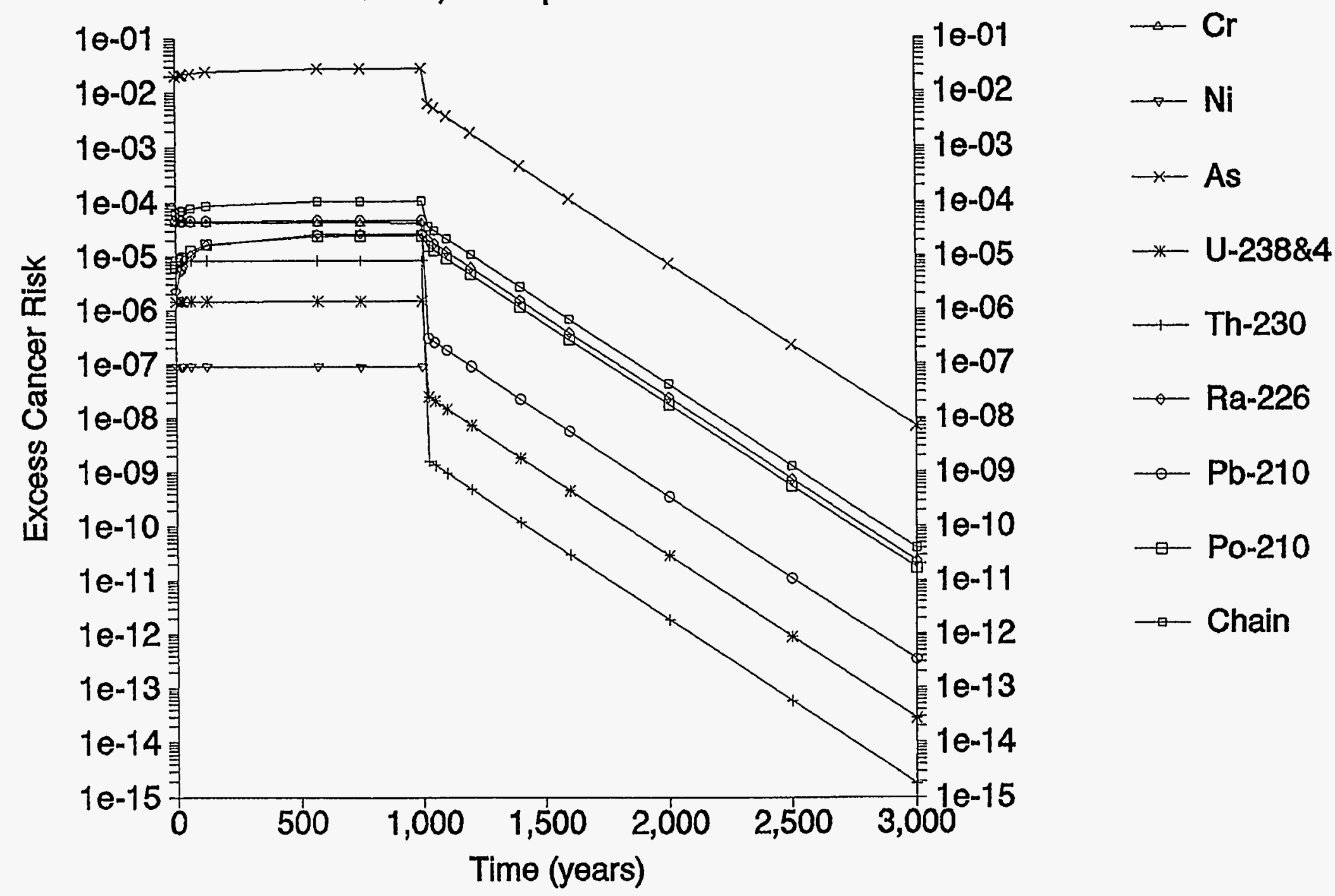

FIGURE 24 Excess Cancer Incidence Risk from Radionuclides at Point of Exposure and from Stable Elements for Atmospheric Release 


\section{DISCUSSION}

\subsection{DEVIATIONS FROM SPECIFIED SCENARIO}

This scenario did not conform exactly to the conceptual model of RESRAD. Some of these deviations were accommodated as described in Chapter 2. The requirements of the scenario that could not be satisfied are discussed in the following paragraphs.

\subsubsection{Off-Site Accumulation in Soil and Resuspension}

As discussed in Section 2.2.4, RESRAD version 5.13 does not model off-site accumulation. Although a methodology to compute the off-site accumulation resulting from atmospheric deposition was developed, a corresponding methodology for accumulation due to irrigation had not been developed at the time the RESRAD calculations were performed. Hence, the external radiation dose and the contribution to root uptake from accumulation due to irrigation are not included in these results. The resuspension of contaminated dust from the off-site location was not modeled for either of the releases.

\subsubsection{Radon}

The radon pathway of RESRAD consists of two subpathways: (1) the diffusion of radon that was generated in the contaminated zone through the uncontaminated cover and the floor or walls of the dwelling, and (2) the release of radon from household water. The scenario specifies the radon release rate from the pile to the atmosphere, and the model is expected to determine the ingrowth during off-site transport and dilution due to dispersion. Because RESRAD version 5.13 is an on-site model and does not compute these effects, the RESRAD radon output was disregarded.

\subsubsection{Aquatic System}

The aquatic system of this scenario consists of a river feeding into a lake. The characteristics of the lake sediments are specified. Lake-sediment interactions and atmospheric deposition of contaminants into the lake are not considered in RESRAD version 5.13. Only the dilution due to the river was considered in determining the contaminant concentration in the lake. 


\subsubsection{Dispersion}

The scenario specifies a (longitudinal) dispersion coefficient, $D_{L}$, of $10^{-3} \mathrm{~m}^{2} \mathrm{~s}^{-1}$. RESRAD version 5.13 does not consider the effects of longitudinal dispersion. A transverse dispersion of one-tenth of the longitudinal dispersion was assumed in the computation of the dilution factor according to Equation K.15 of $\mathrm{Yu}$ et al. (1993). Neglecting the longitudinal dispersion will overestimate the breakthrough time of the contaminants. The peak concentration of the slow-moving contaminants, As and Th-230, will also be overestimated.

\subsection{SUGGESTIONS TO EXTEND CAPABILITY OF RESRAD}

The RESRAD code now can be used to calculate the concentration of contaminants in different media and the radioactive dose attributable to each nuclide at the point of exposure. An offsite residence and cultivation model is also under development. The factors affecting the foliar interception of dust and irrigation water should be amenable to change by the user. The off-site model must include the accumulation of contaminants from atmospheric deposition and contaminated irrigation. The effect of longitudinal dispersion needs to be considered, especially for slow-moving contaminants. The ability to handle a constant release should be provided. The capability to model a step change in the release (could be a decrease due to remediation or could be a truncation due to physical removal of contamination) is essential to analyze different remediation alternatives; this capability should be made available in RESRAD. 


\section{REFERENCES}

BIOMOVS II, 1995, Long Term Contaminant Migration and Impacts from Uranium Mill Tailings: Comparison of Computer Models Using a Hypothetical Dataset, Technical Report 4, published by the Swedish Radiation Protection Institute for the BIOMOVS II Steering Committee, Stockholm, Sweden, Nov.

Yu, C., et al., 1993, Manual for Implementing Residual Radioactive Material Guidelines Using RESRAD, Version 5.0, ANL/EAD/LD-2, Argonne National Laboratory, Argonne, Ill., Sept. 


\section{APPENDIX A:}

\section{SCENARIO DESCRIPTION*}

* Excerpted from the BIOMOVS II Technical Report 4, Long Term Contaminant Migration and Impacts from Uranium Mill Tailings: Comparison of Computer Models Using a Hypothetical Dataset (BIOMOVS II 1995, pp. A1-A13), with permission of the BIOMOVS II Steering Committee. 


\title{
Appendix A: Uranium Mill Tailings Scenario Description V1.07
}

\author{
URANIUM MILL TAILINGS \\ Scenario Description V1.07, April 1993
}

The following sets out the V1.07 scenario as provided to calculating participants. Where clarifications have occurred since, these are noted in italics.

\section{A1. Background}

It is not technically feasible to extract all the uranium during processing of uranium ore. The $U$ content of the resulting tailings typically varies between $0.001 \%$ and $0.01 \%$ and there are associated amounts of toxic elements. The tailings are typically disposed of in the vicinity of the mine or pit, and constitute a potential source of gaseous contaminants (radon) and non-gaseous contaminants including radio elements, such as uranium, thorium, radium and other toxicants such as heavy metals. Both types of release may be important in both the short and long term.

The prediction of transport of radioactive and non-radioactive contaminants from uranium mill tailings into the surrounding environment requires a good understanding of the processes controlling their release and the pathways along which they move. These include release of gases and particulates to air and leaching of contaminants from the tailings into groundwater, rivers, or lakes and their uptake and distribution in biota, soils and sediment.

\section{A2. Objectives}

The overall objectives of the Uranium Mill Tailings Working Group are two-fold.

The first is to compare existing models which can be used to assess the long term impact of radioactive releases from uranium mill tailings, involving multiple pathways, multiple contaminants and multiple receptors with a view to understanding the reasons for the differences in model predictions, including uncertainty.

The second objective is to examine how these models can be used to assess the fate of some stable toxic elements in addition to the radionuclides. Consideration is limited to the radiation doses and risks from the U-238 decay chain, since commonly this dominates over the U-235 chain in tailings assessment results and to the chemically induced cancer risk due to intake of 3 stable elements, arsenic, chromium and nickel. 
BIOMOVS II

TR4

\section{A3. Scope and Work Programme}

To achieve the overall objectives described above a step-wise approach has been adopted that starts with a simplified basic case to which the complexities of the real system will be gradually introduced.

In the first step a basic scenario is defined (see section A4). Model results produced according to this description are used as the basis for intercomparison of the models (Type B approach). The source term from the tailings is treated as a simple constant release for a fixed period so that effort can be concentrated on modelling the subsequent transport in the biosphere. Separate consideration is given to the atmospheric and groundwater release source terms. It is anticipated that comparison of the model results will allow differences arising from model structure to be identified and quantified. Values of important parameters are specified in the basic scenario in order to limit the uncertainties arising due to the user's interpretation. Results are to be calculated on a deterministic basis.

In subsequent steps, it is intended that consideration will be given to probabilistic evaluation of the uncertainties and time dependent source terms. Initially, these tests will be carried out as type B tests. However, data are now sought from a variety of sites around the world, representative of different environments, to provide independent datasets for environmental transport model testing (Type A testing). This should permit comparison of the model results taking account of realistic time dependent source terms. (In fact, no suitable datasets have been identified to date.)

\section{A4. Basic Scenario Description V1.07}

Radioactive and stable toxic contaminants are released continuously for a period of 1000 years from a uranium mill tailings pile into both the atmosphere and the underlying groundwater. After 1000 years the releases stops.

There is an atmospheric release to an agricultural area and a forested area, (see Figure A1). Two zones can be distinguished; zone A which extends from the source (uranium mill tailings pile) out to $5 \mathrm{~km}$, and zone $B$ which extends from $5 \mathrm{~km}$ out to $10 \mathrm{~km}$. Contaminants are also released from the uranium mill tailings pile via groundwater and transported to a small river and well that are situated at $1000 \mathrm{~m}$ (see Figure A2).

The river flows into a lake that is situated in the agricultural area at the boundary between zones $A$ and $B$. There are fish in the lake which are consumed by the populations in both zone A and B. Two crops are grown, leafy vegetables (lettuce) and pasture grass. Beef cattle graze the pasture grass. In zone A one hectare of leafy vegetables (lettuce) is grown at the mid-point $(2.5 \mathrm{~km})$. The lettuce is irrigated with water from the well. The remaining area is pasture grass and is not irrigated. The Zone B agricultural area is divided into two equal areas of leafy vegetables (lettuce) and pasture grass (see Figure 1). Only the crop of leafy vegetables (lettuce) is irrigated, during the summer months with water from the lake. Drinking water (for livestock and humans), is taken from the well in zone A and the lake in zone B. 
For zone A the occupancy is assumed to be at $2.5 \mathrm{~km}, 60 \%$ indoors and $40 \%$ outdoors in the vegetable production area. For zone B it is the same but at $7.5 \mathrm{~km}$.

Full details of the source term and the site characteristics are given in the Annex to the scenario description.

\section{A5. Material Requested from Participants}

Assuming the characteristics specified in the following Annex for the various scenario components, the following calculations are requested, separately for the atmospheric and groundwater source terms. Please present results as a function of time from the commencement of the release. Please truncate the calculation at 10,000 years, even if the system has not come into equilibrium. This truncation is included since beyond that time the whole surface environment will have been altered significantly.

For zone A calculate the concentration of each contaminant due to each source term in:

- atmosphere (Bq $\left.\mathrm{m}^{-3}, \mathrm{mg} \mathrm{m}^{-3}\right)$

- well water (Bq $\left.\mathrm{l}^{-1}, \mathrm{mg} \mathrm{l}^{-1}\right)$

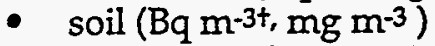

- pasture (Bq kg-1 $\left.\mathrm{ww}^{*}, \mathrm{mg} \mathrm{kg}^{-1} \mathrm{ww}\right)$

- lettuce (Bq kg-1 $\left.\mathrm{ww}^{\mathrm{m}} \mathrm{mg} \mathrm{kg-1}^{-1} \mathrm{ww}\right)$

- beef (Bq kg-1 ww, mg kg-1 ww) and

- fish (Bq kg-1 ww, $\left.\mathrm{mg} \mathrm{kg}^{-1} \mathrm{ww}\right)$

*ww is wet weight tSee Annex for soil data.

For zone B calculate the concentration of each contaminant due to each source term in:

- river water (Bq l-1, $\mathrm{mg} \mathrm{l}^{-1}$ )

- lake water (Bq $\left.\mathrm{l}^{-1}, \mathrm{mg} \mathrm{l}^{-1}\right)$

- lake sediment (Bq kg-1 $\left.\mathrm{ww}^{*}, \mathrm{mg} \mathrm{kg}^{-1} \mathrm{ww}\right)$

- $\operatorname{soil}\left(\mathrm{Bq} \mathrm{m}-3, \mathrm{mg} \mathrm{m}^{-3}\right)$

- pasture (Bq kg-1 ww, mg kg-1 ww)

- lettuce ( $\left.B q \mathrm{~kg}^{-1} \mathrm{ww}, \mathrm{mg} \mathrm{kg}^{-1} \mathrm{ww}\right)$ and

- beef (Bq kg-1 ww, $\left.\mathrm{mg} \mathrm{kg}^{-1} \mathrm{ww}\right)$

For each zone, calculate for each source term:

i. the annual individual effective dose $\left(S v a^{-1}\right)$ due to the release of each radionuclide in the U-238 decay series (defined in the Appendix) for each exposure pathway to members of the critical group. The exposure pathways include: ingestion of drinking water, fish, beef and lettuce; external irradiation from the ground; and inhalation of contaminants from the atmospheric source term and suspended from the ground. Factors to convert annual intake into effective dose are provided in the annex; 
BIOMOVS II

TR4

ii. as for i. but summed over the pathways;

iii. the intake of each stable element ( $\left.\mathrm{mg} \mathrm{a}^{-1}\right)$ for each pathway;

iv. as for iii. but summed over pathways;

v. lifetime average cancer incidence risk from a years exposure or intake summed over pathways for the U-238 series, and for each stable element. Factors to convert annual intake ( $\mathrm{Bq} \mathrm{a}^{-1}$ or $\left.\mathrm{mg} \mathrm{a}^{-1}\right)$ into this quantity are provided in the annex. (The breakdown of risks among pathways can be derived from the other results provided.)

Please provide results as hard copy and on disk in file formats as given in annex.

Participants are also requested to provide:

a one page description of the application of their model to the scenario, noting any ways in which they added to or had to modify the description or site characteristics;

a diagram indicating the components of the model and contaminant transfers;

a brief commentary on the results produced; and

a reference for the model used.

Again, please provide results as hard copy and on disk. Mac disks and Word Perfect are most convenient for the secretariat but PC disks and other word processors would still be very helpful.

In preparing results, participants may wish to take account of the following factors which arise from the scenario description. For the groundwater release, the concentration of contaminants in the leachate is not diluted by mixing with other waters below the tailing pile. Therefore, eventually, the concentrations of long-lived radionuclides and stable contaminants found in the well water could rise to near to the levels in the tailing leachate, although lateral dispersion may reduce these levels by the time the water reaches the well. On entering the river, the leachate is diluted by a factor of ten, so that the long term rise in the river water concentrations would be no more than to one tenth the initial leachate concentration. Concentrations in the lake would rise to similar levels, although there may be additional reductions from the water column due to sedimentation. The water used for well irrigation is given to have no particulate in it. That taken from the lake would have a proportion of activity associated with suspended sediment. Irrigated areas would see an accumulation per $\mathrm{m}^{2}$ corresponding to the rate of irrigation $\left(\mathrm{m} \mathrm{a}^{-1}\right)$ and the concentration in that water. Given the 100 year half-life in soil, concentrations in soils may be expected to rise for several hundred years and then level off. For the atmospheric source term, the distances of special interest with respect to atmospheric dispersion are 2.5 and $7.5 \mathrm{~km}$, since these are the given locations of the critical groups. Deposition onto ground surfaces can result in accumulation of longlived and stable contaminants, so that, in the long term, resuspension may contribute significantly to concentrations in air. 
Annex to Appendix A: Specification of the Source Term and Receiving Environment Characteristics and Other Data Required

\section{A6. Groundwater Release}

A one dimensional model is to be used to calculate the groundwater transport of the contaminants from the urarium mill tailings pile (Figure A2). A simple source term for groundwater release is assumed: a constant concentration of contaminants in the porewater in the tailings. The characteristics of the tailings pile and underlying sandy soil are quantified below.

Uranium Tailings Pile

$\begin{array}{lll}\text { Surface area } & \mathrm{A} & =5105 \mathrm{~m}^{2}, \\ \text { Height } & \mathrm{H} & =10 \mathrm{~m} \\ \text { Infiltration } & \mathrm{I} & =0.1 \mathrm{~m} \mathrm{a}-1\end{array}$

(These data were retained from the previous scenario description version, V1.06. In fact, given the V1.07 groundwater release source term description above, they are redundant and were confusing to some participants.)

Tailings/Sandy Soil

Darcy velocity
Dispersion coefficient
Dry bulk density

Porosity (total effective)

Soil distribution coefficient

$$
\begin{array}{lll}
\mathrm{k} & = & 1.210^{2} \mathrm{~m}^{3} \mathrm{~m}^{-2} \mathrm{a}-1 \\
\mathrm{D}_{\mathrm{L}} & = & 110^{-3} \mathrm{~m}^{2} \mathrm{~s}^{-1} \\
\rho_{\mathrm{b}} & = & 1.410^{3} \mathrm{~kg} \mathrm{~m}^{-3} \\
\varepsilon & = & 40 \% \text { by volume } \\
\mathrm{Kd} & = & \text {. See Table A2 }
\end{array}
$$

Table AI: Source Term Values for Groundwater and Atmospheric Release

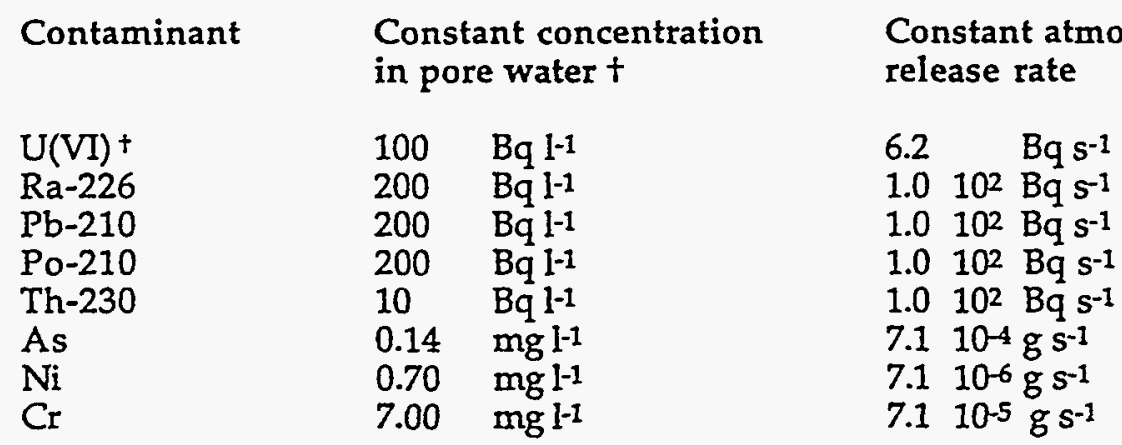

t Please assume $100 \mathrm{~Bq}{ }^{-1}$ and $6.2 \mathrm{~Bq} \mathrm{~s}^{-1}$ each of U-238 and of U-234. For the timescales of interest in this study, these two radionuclides can be assumed to be in equilibrium in all parts of the environment. Results for concentrations of U-234 do not therefore need to be separately presented. Results for the doses for the two radionuclides can be added together, under the heading $U-238 / 4$, noting that the dose and risk factors for the two radionuclides are very similar. Note that the tailings are depleted in uranium relative to the daughters. 
BIOMOVS II

TR4

Table A2: Kd Values to be Assumed for Sandy Soil

Element

$\mathrm{Kd}\left(\mathrm{m}^{3} \mathrm{~kg}^{-1}\right)$

Element

$\mathrm{Kd}\left(\mathrm{m}^{3} \mathrm{~kg}^{-1}\right)$

U

$\mathrm{Ra}$

$\mathrm{Pb}$

Po

Th
$510-3$

$510-2$

$510-2$

$510-2$

1100
$\mathrm{Ni}$

$\mathrm{Cr}$

As
$110-3$

$110-3$

$510-1$

\section{A7. Atmospheric Release}

A constant solid emission rate from the tailings pile due to wind erosion is assumed for the atmospheric release. The atmospheric release rates are summarized in Table A1. For Rn-222 the release is not related to wind erosion. An emission rate of $2.510^{6} \mathrm{~Bq} \mathrm{~s}^{-1}$ for $\mathrm{Rn}-222$ is assumed.

The following conditions are assumed during atmospheric release:

Wind Rose Stability category D

Average windspread $3 \mathrm{~m} \mathrm{~s}^{-1}$

Release height $10 \mathrm{~m}$

Release to 16 sector window $\left(22.5^{\circ}\right)$

Uniform wind rose (ie wind blows equally in all directions).

Radon daughters sorbed on the Aitken nuclei:

Deposition velocity

$$
\mathrm{V}_{\mathrm{gr}}=10^{-3} \mathrm{~m} \mathrm{~s}^{-1}
$$

Soil resuspended particles:

$$
\begin{aligned}
& \text { Resuspension layer } \\
& \text { Resuspension factor } \\
& \text { Deposition velocity }
\end{aligned}
$$

$$
\begin{aligned}
& \mathrm{R}_{1}=10^{-2} \mathrm{~m} \\
& \mathrm{R}_{\mathrm{fs}}=10^{-9} \mathrm{~m}^{-1} \\
& \mathrm{~V}_{\mathrm{gs}}=10^{-2} \mathrm{~ms}^{-1}(-10 \mu \mathrm{m} \text { median } \\
& \text { aerodynamic diameter })
\end{aligned}
$$

\section{A8. Aquatic System}

- A lake is situated in the agricultural area between zone $A$ and $B$ and becomes contaminated through the river (and by aerosols) (Figure Al). Its physical characteristics which are presented below, are the same as those described in BIOMOVS I, Technical Report 1 scenario B3. All the fish eaten by the people living in the two zones are obtained from this lake.

- the lake characteristics

Dilution factor $\left(D_{f}\right)$, due to the river

between the source term and the lake

Volume of the lake

101

Average depth of the lake

$107 \mathrm{~m}^{3}$

Output flow $=$ input flow

$10^{1} \mathrm{~m}$

$10^{8} \mathrm{~m}^{3} \mathrm{a}^{-1}$ 
Net evaporation rate

Suspended sediment load

Suspended sediment $\mathrm{Kd}$

* Net sediment deposition rate through

water column
$0 \mathrm{~m} \mathrm{a}^{-1}$

$10 \mathrm{~g} \mathrm{~m}^{-3}$

Soil $\mathrm{Kd}$ values $\times 10^{2}$

$0.1 \mathrm{~m} \mathrm{~d}^{-1}$

* For the purposes of this exercise the rate of sediment accumulation in the lake affecting hydraulic residence time will not be considered.

- the upper sediments (aerobic) of the lake

Aerobic sediments Kd

Porosity

Depth

Bulk density

Diffusion coefficient
Soil $\mathrm{Kd}$ values $\times 101$

$95 \%$ by volume

$10^{-1} \mathrm{~m}$

$1.0510^{3} \mathrm{~kg} \mathrm{~m}^{-3}$

$10^{-11} \mathrm{~m}^{2} \mathrm{~s}^{-1}$

- the deeper sediments (araerobic) of the lake

Anaerobic sediments $\mathrm{Kd}$

Porosity

Depth

Bulk density

Diffusion coefficient
Soil $\mathrm{Kd}$ values $\times 10^{4}$

$90 \%$ by volume

$510^{-1} \mathrm{~m}$

$1.110^{3} \mathrm{~kg} \mathrm{~m}^{-3}$

$510^{-12} \mathrm{~m}^{2} \mathrm{~s}^{-1}$

- A well is situated in the agricultural area in zone $A, 1000 \mathrm{~m}$ from the uranium tailings release point (Figure A1). The well has no impact on the groundwater discharge to the river and the well water contains no particulates. The well water is used for drinking, and irrigation of 1 ha of agricultural land situated at the midpoint of zone $\mathrm{A}(2.5 \mathrm{~km})$.

\section{A9. Agricultural Area}

For the winter 6 months of the year there is no vegetation. In the summer 6 months, crops are grown. In zone $A$ water is taken from the well to irrigate a crop of leafy vegetables (lettuce) covering 1 hectare at the midpoint $(2.5 \mathrm{~km})$ of zone $A$. The remaining area is devoted to pasture which is not irrigated.

In zone B irrigation water is drawn from the lake and is used to irrigate the crop of leafy vegetables (lettuce) only. The pasture is not irrigated. The soil properties in the agricultural area are given in section $A 6$ (groundwater release). The additional input data are listed below.

Sandy Soil

Ploughing depth

Ploughing frequency

Half life of contaminants in soil due to all removal processes except radioactive decay

$\begin{array}{lll}D_{\mathrm{s}} & = & 0.25 \mathrm{~m} \\ M & = & 1 \mathrm{a}-1 \\ & & \\ S_{v} & =100 \mathrm{a}\end{array}$

A7 
BIOMOVS II

TR4

\section{Crop Data}

Two crops are considered, pasture grass and leafy vegetables. The pasture grass is cut once each season. A 180 day vegetation period is assumed. The vegetation period for leafy vegetables (lettuce) is 90 days. The crop parameters are as follows:

$\begin{array}{lllll}\text { Crops } & \mathrm{Y} & \mathrm{N}_{\mathbf{s}} & \mathrm{T}_{\mathbf{v}} & \mathrm{N}_{\mathbf{l}} \\ \text { Leafy vegetables (lettuce) } & 1.4 & 0.7 & 14 & 0.1 \\ \text { Pasture grass } & 1.85 & 0.5 & 14 & 0.1\end{array}$

where:

$Y$ annual yield ( $\mathrm{kg} \mathrm{m}^{-2}$ wet weight)

$N_{s}$ foliar interception fraction for dust (-)

$T_{v}$ weathering half life for intercepted dust (d)

$\mathrm{N}_{\mathrm{l}}$ foliar interception fraction for irrigation water $(-)$

Irrigation

Those crops which are irrigated are assumed to be irrigated throughout their vegetation period.

Irrigation rate $11 \mathrm{~m}^{-2} \mathrm{~d}^{-1}$

Irrigation time 8 hours per day

The transfer of individual elements from soil to crops via root uptake is specified with a transfer factor $\left(\mathrm{TF}_{\mathrm{SP}}\right)$. The transfer factors to be used are listed in Table 3 and are expressed on a fresh weight plant per dry weight soil basis.

Table A3: Soil-to-Plant Transfer Factors

$\begin{array}{llll}\text { Element } & \mathrm{TF}_{\mathrm{SP}} & \text { Element } & \mathrm{TF}_{\mathrm{SP}} \\ \mathrm{U} & 410^{-3} & \mathrm{Ni} & 510^{-3} \\ \mathrm{Ra} & 510^{-2} & \mathrm{Cr} & 110^{-2} \\ \mathrm{~Pb} & 210^{-3} & \mathrm{As} & 410^{-3} \\ \mathrm{Po} & 510^{-3} & & \\ \mathrm{Th} & 410^{-4} & & \end{array}$

\section{Animal Data}

There is permanent pasture in each zone of the agricultural area and livestock are not transferred between zones. Drinking water for the animals is taken from the well in zone $A$ and from the lake in zone $B$.

Consumption Values (beef cattle)

$\begin{array}{llll}\text { Pasture grass } & 50 & \mathrm{~kg} \mathrm{~d}^{-1} & \text { (wet weight) } \\ \text { Soil } & 0.5 & \mathrm{~kg} \mathrm{~d}^{-1} & \text { (wet weight) } \\ \text { Water } & 50 & 1 \mathrm{~d} \mathrm{~d}^{-1} & \end{array}$


Inhalation rate $\quad 150 \quad \mathrm{~m}^{3} \mathrm{~d}^{-1}$

The transfer of individual elements to beef and edible portion of fish following ingestion is quantified by a distribution factor (DF). The distribution factors for meat and fish are summarised in Table A4 and are expressed on a wet weight basis.

Table A4: Animal Distribution Factors

\begin{tabular}{|c|c|c|}
\hline Element & $D F_{m}\left(d k g^{-1}\right)$ meat & $D F_{f}\left(1 k^{-1}\right)$ fish \\
\hline $\mathrm{U}$ & $310^{-2}$ & 1101 \\
\hline $\mathrm{Ra}$ & $510^{-4}$ & 5101 \\
\hline $\mathrm{Pb}$ & $210^{-3}$ & $310^{2}$ \\
\hline Po & $510^{-1}$ & $510^{1}$ \\
\hline Th & $410^{-4}$ & $110^{2}$ \\
\hline As & $510^{-1}$ & 5101 \\
\hline $\mathrm{Ni}$ & $510^{-3}$ & 5101 \\
\hline $\mathrm{Cr}$ & $110^{-2}$ & $210^{2}$ \\
\hline
\end{tabular}

(Subsequent review of this choice of parameters highlighted that the assumption for Po, 0.5 $d \mathrm{~kg}^{-1}$, is very high. More typically a value two orders of magnitude lower is assumed, eg see LAEA Safety Series 57.)

\section{A10. Human Data}

The consumption values and percentage occupancy for individuals in the different regions of each zone are listed below. It is assumed that the critical group members are permanently resident in their respective zones. The exposure pathways to be considered are inhalation, ingestion and external exposure.

Diet: meat (beef)

fish vegetables (lettuce)

drinking water

$50 \mathrm{~kg} \mathrm{a}^{-1}$

$5 \mathrm{~kg} \mathrm{a}-1$

$25 \mathrm{~kg} \mathrm{a}^{-1}$

$21 d^{-1}$

Occupancy: home

$60 \%$

farmland

$40 \%$

Indoor reduction of dust relative to outside

$50 \%$

Shielding effect of being indoors relative to outside

$50 \%$

Indoor radon equilibrium factor

0.5

Outdoor radon equilibrium factor

Zone A

Zone B

0.1

Inhalation rate

0.3

$8400 \mathrm{~m}^{3} \mathrm{a}^{-1}$ 
BIOMOVS II

$T R 4$

\section{A11. Factors for Converting Annual Intake to Radiation Dose}

$\begin{array}{lll} & \begin{array}{l}\text { Inhalation } \\ \text { Sv Bq-1 }\end{array} & \begin{array}{l}\text { Ingestion } \\ \text { Sv Bq-1 }\end{array} \\ \mathrm{U}-238 & 3.1 \mathrm{E}-5 & 4.2 \mathrm{E}-8 \\ \mathrm{U}-234 & 3.5 \mathrm{E}-5 & 3.9 \mathrm{E}-8 \\ \mathrm{Th}-230 & 5.1 \mathrm{E}-5 & 3.5 \mathrm{E}-7 \\ \mathrm{Ra}-226 & 2.1 \mathrm{E}-6 & 2.2 \mathrm{E}-7 \\ \mathrm{Rn}-222^{*} & 1.2 \mathrm{E}-8 & \text { Not applicable } \\ \mathrm{Pb}-210 & 2.2 \mathrm{E}-6 & 1.3 \mathrm{E}-6 \\ \mathrm{Po}-210 & 1.9 \mathrm{E}-6 & 6.2 \mathrm{E}-7\end{array}$

Effects of unlisted daughters are included with those of the parents, on the assumption that they must be in equilibrium with the parent at the time of intake. Values listed are committed effective doses to adults for the chemical form giving rise to the highest dose in each case. Data have been taken from Phipps A W, Kendall G M, Stather J W and Fell T P, Committed Equivalent Organ Doses and Committed Effective Doses from Intakes of Radionuclides. National Radiological Protection Board, NRPB-R245, London, HMSO, 1991.

* R245 does not give numbers for $R n-222$. The value for $R n-222$ is derived from the NRPB Board Statement on Radon in Homes, Documents of the NRPB, volume 1, no1, Chilton, 1990, and is the value of dose arising from Rn-222 and its solid shortlived decay products per $\mathrm{Bq}$ of $\mathrm{Rn}-222$ inhaled. If additional or alternative radon dosimetry is adopted, eg involving the radon equilibrium factors listed above, please indicate this with your results.

\section{A12. Factors for Converting Annual Intake to Risk}

The radionuclide data given below are taken from Health Effects Assessment Summary Tables, Annual FY 1992, United States Environmental Protection Agency, OERR 9200.6-303 (92-1). They represent the lifetime total excess cancer risk per unit intake or exposure. If the intake occurs over one year, then risk calculated is the lifetime risk from that year's intake. Likewise, for unit activity concentration in the soil, then the figures represent the lifetime risk from one year of exposure. (These numbers will not necessarily give the same risk as would be obtained by multiplying the calculated effective dose by the risk per unit effective dose, eg as assessed by ICRP.)

$\mathrm{U}-238$

Inhalation

Ingestion

External

$\mathrm{U}-234$

Risk/Bq

Risk/Bq

$1.4 \mathrm{E}-6$

7.6E-10

Risk/y per Bq/g soil

Th-230

7.0E-7

$7.8 \mathrm{E}-7$

Ra-226

8.1E-8

4.3E-10

3.5E-10

9.7E-7

$\mathrm{R} n-222$

2.1E-10

3.2E-9

8.1E-10

$1.5 \mathrm{E}-9$

$\mathrm{Pb}-210$

3.5E-8

4.6E-11

$1.6 \mathrm{E}-4$

$1.4 \mathrm{E}-8$

$1.6 \mathrm{E}-4$

Po-210

7.0E-8

4.1E-9

7.8E-10

Effects of unlisted daughters are included with those of the parents. In the case of $\mathrm{Ra}-226$ it is assumed that $\mathrm{Rn}-222$ and its short-lived daughters are also present in equilibrium on intake with the Ra-226. 
The following data for metals are also taken from the EPA report. The values represent the same cancer risk as above per $\mathrm{mg}$ intake assuming that intake is evenly distributed throughout a year, and so represents the risk per unit annual intake.

$\begin{array}{lll} & \text { Inhalation } & \text { Ingestion } \\ & \text { Risk/mg } & \text { Risk/mg } \\ \text { As } & 6.8 \mathrm{E}-3 & 2.5 \mathrm{E}-4 \\ \mathrm{Cr} & 5.6 \mathrm{E}-3 & - \\ \mathrm{Ni} & 1.2 \mathrm{E}-4 & -\end{array}$

(Risk data of this type are constantly under reoiew, though the basic data for these three elements are unchanged in the 1994 version of the EPA's HEAST tables. The extension of the HEAST carcinogeneity data, based on continuous lifetime exposure, to lifetime risk from an animal intake may be questioned. However, the illustration of the type of calculation which can be done is interesting.)

\section{A13. Requested Format for Results Files}

To assist in the collation of results it is suggested that a common file name structure should be used for the results files, as follows.

1st character identifies atmospheric or groundwater source term, A or G

2nd character, zone A or zone B

A or B

3rd character, the medium;

$\begin{array}{ll}\text { atmosphere } & \text { A } \\ \text { soil } & \text { S } \\ \text { pasture } & \text { P } \\ \text { lettuce (vegetable) } & \text { V } \\ \text { beef } & \text { B } \\ \text { fish } & \mathrm{F} \\ \text { river water } & \mathrm{R} \\ \text { well water } & \text { W } \\ \text { lake water } & \mathrm{L} \\ \text { lake sediment (mud) } & \mathrm{M} \\ \text { sum over pathways (total) } & \mathrm{T}\end{array}$

4th character, concentration, intake, dose or risk $\quad$ C, I, D, or R

5th character, contaminant:
U-238 chain
Metals
U

As examples, the dose from the U-238 chain due to ingestion of lettuce in zone A due to the atmospheric source term would be in file AAVDU.data. The metals intake in zone $B$ from all pathways from the groundwater source term for the whole chain would be in file GBTIM. The following is the suggested layout for the data.

Line one. A title line, eg AAVDU, also giving the units in which results are reported. These should be as requested above, but respecifying them is intended to provide an additional check. 
BIOMOVS II

TR4

Line two. A header line as follows for the U-238 chain:

Time y | U-238/4 |Th-230 |Ra-226 | Pb-210 |Po-210 |Rn-222 Ichain sum

The U-238/4 column should contain the contributions from both radionuclides. In the case of activity, the amounts for each radionuclide are the same. In the case of dose or risk the amounts for each radionuclide are similar. Rn-222 results are put to the end since they are not required for all cases, eg for the groundwater release.

For the metals line 2 becomes:

Time y / arsenic /chromium / nickel

Line three. The results at each time, eg

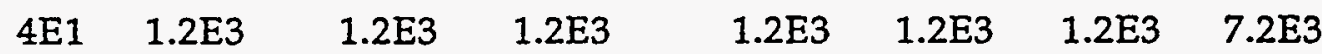


BIOMOVS II

TR4

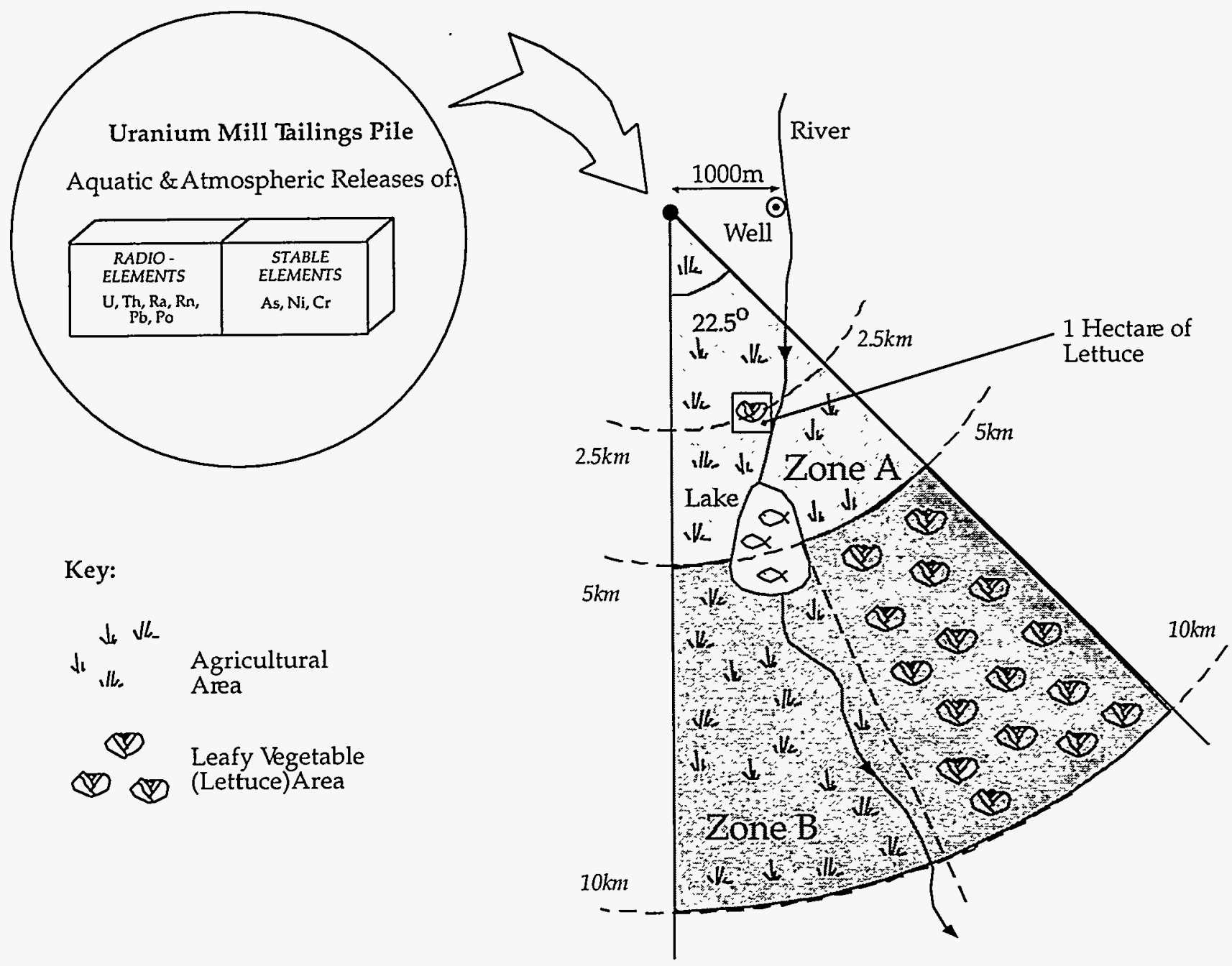

Figure A1: Plan View of Area Surrounding the Uranium Mill Tailings Pile 
APPENDIX B:

OFF-SITE ACCUMULATION MODEL 



\section{APPENDIX B:}

\section{OFF-SITE ACCUMULATION MODEL}

The processes that affect the activity concentration off-site are the following:

- Time-dependent deposition of contaminants,

- Uniform mixing in a mixing zone,

- First-order (radioactive) transformations,

- Surface erosion at a constant rate, and

- Adsorption equilibrium controlled release from the mixing zone.

\section{Dimensions}

Dimensions include length $(L)$, mass $(M)$, time $(T)$, and activity $(A)$.

\section{Terms}
$\mathrm{A}^{\mathrm{o}} \quad$ is the off-site area $L^{2}$;
$\mathrm{D}_{\mathrm{i}}(\mathrm{t}) \quad$ is the deposition rate, $A L^{-2} T^{-1}$, of contaminant $i$;
$\mathrm{d}_{\mathrm{m}}{ }^{\circ} \quad$ is the off-site depth of mixing, $L$;
$\epsilon^{0} \quad$ is the off-site surface erosion rate, $L T^{-1}$;
$\theta^{\circ} \quad$ is the off-site moisture content;
$I^{\circ} \quad$ is the off-site infiltration rate, $L T^{-1}$;
$\mathrm{K}_{\mathrm{d}}^{\mathrm{o}} \quad$ is the off-site distribution coefficient, $L^{-3} M$, of contaminant $i$;
$\mathrm{L}_{\mathrm{i}}^{\circ} \quad$ is the off-site leach rate, $T^{-1}$, of contaminant $i$;
$\lambda_{\mathrm{i}} \quad$ is the transformation coefficient, $T^{-1}$, of contaminant $i$;
$\rho_{b}{ }^{0} \quad$ is the off-site bulk density, $M L^{-3}$;
$\mathrm{R}_{\mathrm{d}}^{0} \mathrm{i} \quad$ is the off-site retardation factor of contaminant $i$; 
$\begin{array}{ll}\mathrm{s}_{\mathrm{i}}^{\mathrm{o}}(\mathrm{t}) \quad \text { is the off-site activity concentration, } A M^{-1} \text {, of contaminant } i \text {; and } \\ \mathrm{t} & \text { is time, } T .\end{array}$

Net Change in Mixing Zone

The net change in the number of atoms of $i$ in the mixing zone between time $t$ and $t+d t$ is

$$
\frac{\rho_{\mathrm{b}}^{\mathrm{o}} \times \mathrm{d}_{\mathrm{m}}^{\mathrm{o}} \times \mathrm{A}^{\mathrm{o}}}{\lambda_{\mathrm{i}}} \mathrm{d}\left[\mathrm{s}_{\mathrm{i}}^{\mathrm{o}}(\mathrm{t})\right]
$$

\section{Deposition}

The number of atoms of $i$ added to the mixing zone because of deposition between time $t$ and $t+d t$ is

$$
\frac{D_{i}(t) \times A^{0} \times d t}{\lambda_{i}}
$$

\section{Radioactive Transformations}

The number of atoms of $i$ added to the mixing zone because of radioactive transformations of $j(=i-1)$, the parent of $i$, between time $t$ and $t+d t$ is

$$
s_{j}^{o}(t) \times \rho_{b}^{o} \times d_{m}^{o} \times A^{o} \times d t
$$

The number of atoms of $i$ removed from the mixing zone because of radioactive transformations of $i$ between time $t$ and $t+d t$ is

$$
s_{i}^{o}(t) \times \rho_{b}^{o} \times d_{m}^{o} \times A^{\circ} \times d t
$$




\section{Surface Erosion}

The number of atoms of $i$ removed from the mixing zone because of surface erosion between time $t$ and $t+d t$ is

$$
\frac{\mathrm{s}_{\mathrm{i}}^{\mathrm{o}}(\mathrm{t}) \times \rho_{\mathrm{b}}^{\mathrm{o}} \times \epsilon^{\mathrm{o}} \times \mathrm{A}^{\mathrm{o}} \times \mathrm{dt}}{\lambda_{\mathrm{i}}}
$$

\section{Adsorption Equilibrium Leaching}

Let $c_{i}(t)$ and $s_{i}{ }^{\prime}(t)$ denote the activity concentrations of nuclide $i$ in the aqueous and solid phases of the mixing zone at time $t$. Then

$$
c_{i}(t) \times \theta^{\circ}+s_{i}{ }^{\prime}(t) \times \rho_{b}^{o}=s_{i}{ }^{o}(t) \times \rho_{b}^{o}
$$

and

$$
s_{i}^{\prime}(t)=c_{i}(t) \times K_{d_{i}}^{o}
$$

Thus,

$$
c_{i}(t)=\frac{s_{i}^{o}(t) \times \rho_{b}^{o}}{\theta^{o}+K_{d_{i}}^{o} \times \rho_{b}^{o}}
$$

The number of atoms of $i$ removed from the mixing zone because of leaching between time $t$ and $t+d t$ is

$$
\begin{aligned}
\frac{c_{i}(t) \times A^{0} \times I^{0} \times d t}{\lambda_{i}} & =\frac{s_{i}{ }^{0}(t) \times \rho_{b}^{0} \times A^{0} \times I^{0} \times d t}{\theta^{0} \times R_{d_{i}}^{0} \times \lambda_{i}} \\
& =\frac{L_{i}^{0} \times s_{i}^{0}(t) \times \rho_{b}^{0} \times d_{m}^{0} \times A^{0} \times d t}{\lambda_{i}},
\end{aligned}
$$


where

$$
\mathrm{L}_{\mathrm{i}}^{0}=\frac{\mathrm{I}^{0}}{\mathrm{~d}_{\mathrm{m}}^{0} \times \theta \times \mathrm{R}_{\mathrm{d}_{\mathrm{i}}}^{0}}
$$

Net Change in Number of Atoms of Nuclide $i$ in Time $d t$

$$
\begin{aligned}
\frac{\rho_{b}^{o} \times d_{m}^{o} \times A^{o}}{\lambda_{i}} d\left[s_{i}^{o}(t)\right]= & \frac{D_{i}(t) \times A^{0} \times d t}{\lambda_{i}}+\left[s_{j}^{o}(t)-s_{i}^{o}(t)\right] \times \rho_{b}^{o} \times d_{m}^{o} \times A^{o} \times d t \\
& -\frac{s_{i}^{o}(t) \times \rho_{b}^{o} \times \epsilon^{o} \times A^{o} \times d t}{\lambda_{i}} \\
& -\frac{s_{i}^{o}(t) \times L_{i}^{o} \times \rho_{b}^{o} \times d_{m}^{o} \times A^{o} \times d t}{\lambda_{i}} .
\end{aligned}
$$

Rearranging and collecting terms,

$$
\mathrm{d}\left[\mathrm{s}_{\mathrm{i}}{ }^{\circ}(\mathrm{t})\right]=\frac{\mathrm{D}_{\mathrm{i}}(\mathrm{t}) \times \mathrm{dt}}{\rho_{\mathrm{b}}^{\mathrm{o}} \times \mathrm{d}_{\mathrm{m}}^{\mathrm{o}}}+\lambda_{\mathrm{i}} \times \mathrm{s}_{\mathrm{j}}{ }^{\mathrm{o}}(\mathrm{t}) \times \mathrm{dt}-\left(\lambda_{\mathrm{i}}+\frac{\epsilon^{\mathrm{o}}}{\mathrm{d}_{\mathrm{m}}^{\mathrm{o}}}+\mathrm{L}_{\mathrm{i}}^{\mathrm{o}}\right) \mathrm{s}_{\mathrm{i}}{ }^{\circ}(\mathrm{t}) \times \mathrm{dt}
$$

Multiplying both sides by

$$
\left.e^{\left(\lambda_{i}+\frac{\epsilon^{0}}{d_{m}^{0}}+L_{i}^{0}\right.}\right) t
$$


and rearranging,

$$
\begin{aligned}
& e^{\left(\lambda_{i}+\frac{\epsilon^{0}}{d_{m}^{0}}+L_{i}^{0}\right)} d\left[s_{i}^{0}(t)\right]+\left(\lambda_{i}+\frac{\epsilon^{0}}{d_{m}^{0}}+L_{i}^{0}\right) e^{\left(\lambda_{i}+\frac{\epsilon^{0}}{d_{m}^{0}+L_{i}^{0}}\right) t} d t \times s_{i}^{0}(t) \\
& =\frac{D_{i}(t) \times d t}{\rho_{b}^{0} \times d_{m}^{0}} \times e^{\left(\lambda_{1}+\frac{\epsilon^{0}}{d_{m}^{0}}+L_{i}^{0}\right) t}+\lambda_{i} \times s_{j}^{0}(t) \times e^{\left(\lambda_{i}+\frac{\epsilon^{0}}{d_{m}^{0}}+L_{i}^{0}\right) t} \times d t \\
& d\left[e^{\left(\lambda_{i}+\frac{\epsilon^{0}}{d_{m}^{0}}+L_{i}^{0}\right) t} \times s_{i}^{0}(t)\right] \\
& =\frac{D_{i}(t) \times d t}{\rho_{b}^{0} \times d_{m}^{0}} \times e^{\left(\lambda_{i}+\frac{\epsilon^{0}}{d_{m}^{0}}+L_{i}^{0}\right) t}+\lambda_{i} \times s_{j}^{0}(t) \times e^{\left(\lambda_{1}+\frac{\epsilon^{0}}{d_{m}^{0}}+L_{i}^{0}\right) t} \times d t
\end{aligned}
$$

Integrating over time from zero to $t$,

$$
\begin{aligned}
& e^{\left(\lambda_{i}+\frac{\epsilon^{0}}{d_{m}^{0}}+L_{i}^{0}\right) t} \times s_{i}^{0}(t)-s_{i}^{0}(0)=\frac{1}{\rho_{b}^{0} \times d_{m}^{0}} \times \int_{0}^{t} D_{i}(t) \times e^{\left(\lambda_{1}+\frac{\epsilon^{0}}{d_{m}^{0}}+L_{i}^{0}\right)} \times d t \\
& +\lambda_{i} \times \int_{0}^{t} s_{j}^{o}(t) \times e^{\left(\lambda_{i}+\frac{\epsilon^{o}}{d_{m}^{o}}+L_{i}^{0}\right) t} \times d t
\end{aligned}
$$


and rearranging,

$$
\begin{aligned}
& s_{i}^{0}(t)=s_{i}^{0}(0) \times e^{-\left(\lambda_{i}+\frac{\epsilon^{0}}{d_{m}^{0}}+L_{i}^{0}\right) t}+\frac{e^{-\left(\lambda_{i}+\frac{\epsilon^{0}}{d_{m}^{0}}+L_{i}^{0}\right) t}}{\rho_{b}^{0} \times d_{m}^{0}} \times \int_{0}^{t} D_{i}(t) \times e^{\left(\lambda_{i}+\frac{\epsilon^{0}}{d_{m}^{0}+L_{i}^{0}}\right) t} \times d t \\
& +\lambda_{i} \times e^{-\left(\lambda_{i}+\frac{\epsilon^{0}}{d_{m}^{0}}+L_{i}^{0}\right) t} \times \int_{0}^{t} s_{j}^{0}(t) \times e^{\left(\lambda_{i}+\frac{\epsilon^{0}}{d_{m}^{0}}+L_{i}^{0}\right) t} \times d t .
\end{aligned}
$$

If $D_{i}(t)$ is independent of time, as in the UMT case, integration yields

$$
\begin{aligned}
& s_{i}^{0}(t)=s_{i}^{0}(0) \times e^{-\left(\lambda_{i}+\frac{\epsilon^{0}}{d_{m}^{0}}+L_{i}^{0}\right) t}+\frac{D_{i} \times\left[1-e^{-\left(\lambda_{1}+\frac{\epsilon^{0}}{d_{m}^{0}}+L_{i}^{0}\right) t}\right]}{\rho_{b}^{0} \times d_{m}^{0} \times\left(\lambda_{i}+\frac{\epsilon^{0}}{d_{m}^{0}}+L_{i}^{0}\right)} \\
& +\lambda_{\mathrm{i}} \times \mathrm{e}^{-\left(\lambda_{\mathrm{i}}+\frac{\epsilon^{0}}{\mathrm{~d}_{\mathrm{m}}^{0}}+L_{\mathrm{i}}^{0}\right)} \times \int_{0}^{\mathrm{t}} \mathrm{s}_{\mathrm{j}}^{0}(\mathrm{t}) \times \mathrm{e}^{\left(\lambda_{\mathrm{i}}+\frac{\epsilon^{0}}{\mathrm{~d}_{\mathrm{m}}^{0}+L_{\mathrm{i}}^{0}}\right) \mathrm{t}} \times d t .
\end{aligned}
$$

\section{First Member of Transformation Chain}

$$
\begin{gathered}
\mathrm{s}_{1}^{0}(\mathrm{t})=\mathrm{s}_{1}^{0}(0) \times \mathrm{e}^{-\left(\lambda_{1}+\frac{\epsilon^{0}}{\mathrm{~d}_{\mathrm{m}}^{0}}+\mathrm{L}_{1}^{0}\right) t}+\frac{\mathrm{D}_{1} \times\left[1-\mathrm{e}^{-\left(\lambda_{1}+\frac{\epsilon^{0}}{\mathrm{~d}_{\mathrm{m}}^{0}}+L_{1}^{0}\right) t}\right]}{\rho_{\mathrm{b}}^{0} \times \mathrm{d}_{\mathrm{m}}^{0} \times\left(\lambda_{1}+\frac{\epsilon^{0}}{\mathrm{~d}_{\mathrm{m}}^{0}}+L_{1}^{0}\right)} \\
\mathrm{s}_{1}^{0}(\mathrm{t})=\mathrm{C}_{1}^{0}+\mathrm{C}_{1}^{1} \mathrm{e}^{-\left(\lambda_{1}+\frac{\epsilon^{0}}{\mathrm{~d}_{\mathrm{m}}^{0}}+L_{1}^{0}\right) t},
\end{gathered}
$$

where

$$
\mathrm{C}_{1}^{0}=\mathrm{s}_{1}^{0}(0)-\mathrm{C}_{1}^{1}=\frac{\mathrm{D}_{1}}{\rho_{\mathrm{b}}^{\mathrm{o}} \times \mathrm{d}_{\mathrm{m}}^{\mathrm{o}} \times\left(\lambda_{1}+\frac{\epsilon^{\mathrm{o}}}{\mathrm{d}_{\mathrm{m}}^{\mathrm{o}}}+\mathrm{L}_{1}^{0}\right)} .
$$


Second Member of Transformation Chain

$$
\begin{aligned}
& \mathrm{s}_{2}{ }^{0}(\mathrm{t})=\mathrm{s}_{2}{ }^{0}(0) \times \mathrm{e}^{-\left(\lambda_{2}+\frac{\epsilon^{0}}{\mathrm{~d}_{\mathrm{m}}^{0}}+L_{2}^{0}\right) t}+\frac{\mathrm{D}_{2} \times\left[1-\mathrm{e}^{-\left(\lambda_{2}+\frac{\epsilon^{0}}{\mathrm{~d}_{\mathrm{m}}^{0}}+L_{2}^{0}\right)} \mathrm{t}\right]}{\rho_{\mathrm{b}}^{0} \times \mathrm{d}_{\mathrm{m}}^{0} \times\left(\lambda_{2}+\frac{\epsilon^{0}}{\mathrm{~d}_{\mathrm{m}}^{0}}+L_{2}^{0}\right)}
\end{aligned}
$$

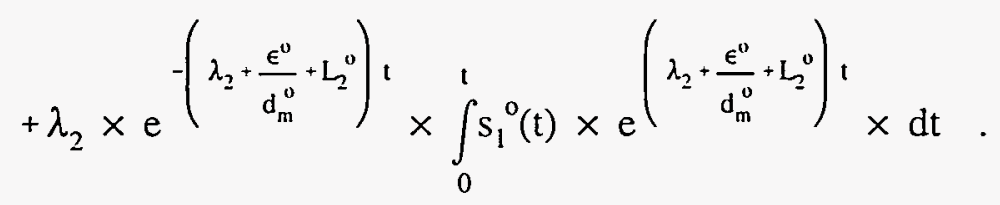

Substituting for $\mathrm{s}_{1}{ }^{\mathrm{o}}(\mathrm{t})$,

$$
\begin{aligned}
& \mathrm{s}_{2}^{0}(\mathrm{t})=\mathrm{s}_{2}^{0}(0) \times \mathrm{e}^{-\left(\lambda_{2}+\frac{\epsilon^{0}}{\mathrm{~d}_{\mathrm{m}}^{\mathrm{o}}}+\mathrm{L}_{2}^{0}\right) \mathrm{t}}+\frac{\mathrm{D}_{2} \times\left[1-\mathrm{e}^{-\left(\lambda_{2} \cdot \frac{\epsilon^{0}}{\mathrm{~d}_{\mathrm{m}}^{\mathrm{o}}}+\mathrm{L}_{2}^{0}\right) \mathrm{t}}\right]}{\rho_{\mathrm{b}}^{\mathrm{o}} \times \mathrm{d}_{\mathrm{m}}^{\mathrm{o}} \times\left(\lambda_{2}+\frac{\epsilon^{0}}{\mathrm{~d}_{\mathrm{m}}^{\mathrm{o}}}+\mathrm{L}_{2}^{\mathrm{o}}\right)}
\end{aligned}
$$

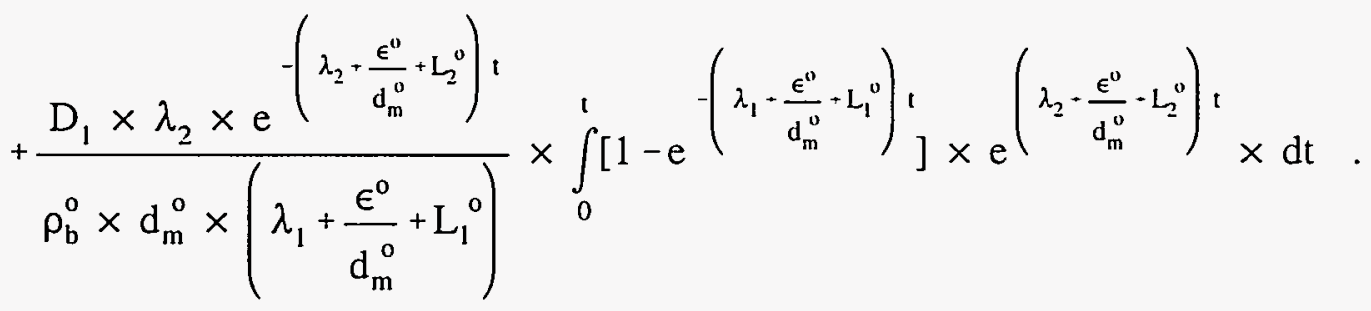


Integrating,

$$
\begin{aligned}
& s_{2}^{0}(t)=s_{2}^{0}(0) \times e^{-\left(\lambda_{2}+\frac{\epsilon^{0}}{d_{m}^{0}}+L_{2}^{0}\right) t}+\frac{D_{2} \times\left[1-e^{-\left(\lambda_{2}+\frac{\epsilon^{0}}{d_{m}^{0}}+L_{2}^{0}\right) t}\right]}{\rho_{b}^{0} \times d_{m}^{0} \times\left(\lambda_{2}+\frac{\epsilon^{0}}{d_{m}^{0}}+L_{2}^{0}\right)}
\end{aligned}
$$

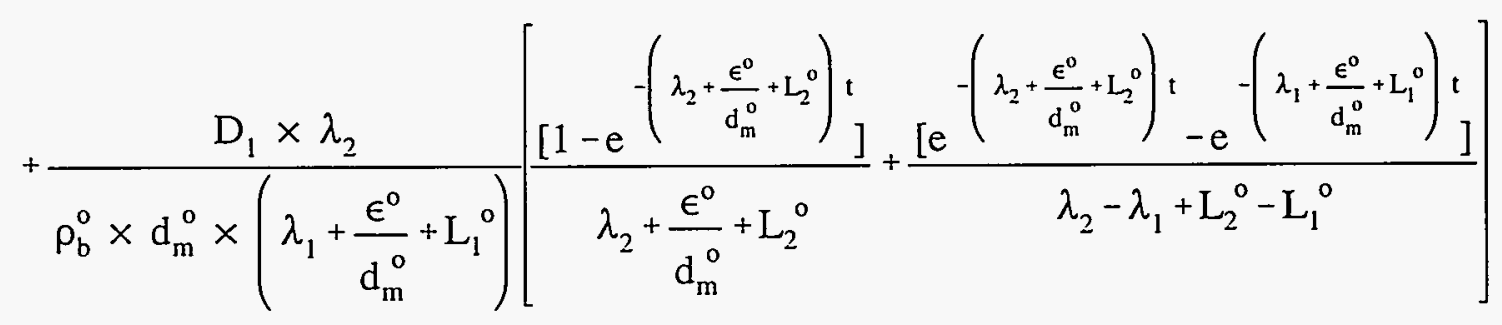

$$
\begin{aligned}
& s_{2}^{0}(t)=C_{2}^{0}+C_{2}^{1} e^{-\left(\lambda_{1}+\frac{\epsilon^{0}}{d_{m}^{0}}+L_{1}^{0}\right) t}+C_{2}^{2} e^{-\left(\lambda_{2}+\frac{\epsilon^{0}}{d_{m}^{0}}+L_{2}^{0}\right) t}
\end{aligned}
$$

where

$$
\begin{aligned}
& \mathrm{C}_{2}^{0}=\frac{\mathrm{C}_{1}^{0} \times \lambda_{2}}{\lambda_{2}+\frac{\epsilon^{\mathrm{o}}}{\mathrm{d}_{\mathrm{m}}^{\mathrm{o}}}+\mathrm{L}_{2}^{\mathrm{o}}}+\frac{\mathrm{D}_{2}}{\rho_{\mathrm{b}}^{\mathrm{o}} \times \mathrm{d}_{\mathrm{m}}^{\mathrm{o}} \times\left(\lambda_{2}+\frac{\epsilon^{\mathrm{o}}}{\mathrm{d}_{\mathrm{m}}^{\mathrm{o}}}+\mathrm{L}_{2}^{\mathrm{o}}\right)} \\
& \mathrm{C}_{2}^{1}=\frac{\mathrm{C}_{1}^{1} \times \lambda_{2}}{\lambda_{2}-\lambda_{1}+\mathrm{L}_{2}^{\circ}-\mathrm{L}_{1}^{\mathrm{o}}} \\
& \mathrm{C}_{2}^{2}=\mathrm{s}_{2}^{\circ}(0) 1-\frac{\mathrm{C}_{1}^{0} \times \lambda_{2}}{\lambda_{2}+\frac{\epsilon^{\mathrm{o}}}{\mathrm{d}_{\mathrm{m}}^{\mathrm{o}}}+\mathrm{L}_{2}^{\circ}}-\frac{\mathrm{C}_{1}^{1} \times \lambda_{2}}{\lambda_{2}-\lambda_{1}+\mathrm{L}_{2}^{\mathrm{o}}-\mathrm{L}_{1}^{\mathrm{o}}}-\frac{\mathrm{D}_{2}}{\rho_{\mathrm{b}}^{\mathrm{o}} \times \mathrm{d}_{\mathrm{m}}^{\mathrm{o}} \times\left(\lambda_{2}+\frac{\epsilon^{\mathrm{o}}}{\mathrm{d}_{\mathrm{m}}^{\mathrm{o}}}+\mathrm{L}_{2}^{\mathrm{o}}\right)} \\
& =s_{2}^{0}(0)-C_{2}^{1}-C_{2}^{0} \text {. }
\end{aligned}
$$


$I^{\text {th }}$ Member of Transformation Chain

$$
\begin{aligned}
& s_{i}^{0}(t)=s_{i}^{0}(0) \times e^{-\left(\lambda_{i}+\frac{\epsilon^{0}}{d_{m}^{0}}+L_{i}^{0}\right) t}+\frac{D_{i} \times\left[1-e^{-\left(\lambda_{i}+\frac{\epsilon^{0}}{d_{m}^{0}+L_{i}^{0}}\right) t}\right]}{\rho_{b}^{0} \times d_{m}^{0} \times\left(\lambda_{i}+\frac{\epsilon^{0}}{d_{m}^{0}}+L_{i}^{0}\right)} \\
& +\lambda_{i} \times e^{-\left(\lambda_{i}+\frac{\epsilon^{0}}{d_{m}^{0}}+L_{i}^{0}\right) t} \times \int_{0}^{t} s_{j}^{0}(t) \times e^{\left(\lambda_{1}+\frac{\epsilon^{0}}{d_{m}^{0}}+L_{i}^{0}\right) t} \times d t
\end{aligned}
$$

If

$$
\mathrm{s}_{\mathrm{j}}{ }^{0}(\mathrm{t})=\mathrm{C}_{\mathrm{j}}^{0}+\mathrm{C}_{\mathrm{j}} \mathrm{e}^{-\left(\lambda_{1}+\frac{\epsilon^{0}}{\mathrm{~d}_{\mathrm{m}}^{0}}+\mathrm{L}_{1}^{0}\right) t}+\mathrm{C}_{\mathrm{j}}^{2} \mathrm{e}^{-\left(\lambda_{2}+\frac{\epsilon^{0}}{d_{m}^{0}+L_{2}^{0}}\right) t}+\ldots+C_{j}^{j} \mathrm{e}^{-\left(\lambda_{1}+\frac{\epsilon^{0}}{d_{m}^{0}}+L_{j}^{0}\right) t},
$$

then

$$
\mathrm{s}_{\mathrm{i}}^{0}(\mathrm{t})=\mathrm{C}_{\mathrm{i}}^{0}+\mathrm{C}_{\mathrm{i}}^{1} \mathrm{e}^{-\left(\lambda_{1}+\frac{\epsilon^{\mathrm{o}}}{\mathrm{d}_{\mathrm{m}}^{0}}+\mathrm{L}_{1}^{0}\right) \mathrm{t}}+\mathrm{C}_{\mathrm{i}}^{2} \mathrm{e}^{-\left(\lambda_{2}+\frac{\epsilon^{0}}{\mathrm{~d}_{\mathrm{m}}^{0}}+\mathrm{L}_{2}^{0}\right) t}+\ldots+\mathrm{C}_{\mathrm{i}}^{\mathrm{i}} \mathrm{e}^{-\left(\lambda_{1}+\frac{\epsilon^{0}}{\mathrm{~d}_{\mathrm{m}}^{0}}+\mathrm{L}_{1}^{0}\right) t},
$$

where

$$
\begin{aligned}
& C_{i}^{0}=\frac{C_{j}^{0} \times \lambda_{i}}{\lambda_{i}+\frac{\epsilon^{0}}{d_{m}^{0}}+L_{i}^{0}}+\frac{D_{i}}{\rho_{b}^{0} \times d_{m}^{0} \times\left(\lambda_{i}+\frac{\epsilon^{o}}{d_{m}^{o}}+L_{i}^{0}\right)}, \\
& C_{i}{ }^{r}=\frac{C_{j}{ }^{r} \times \lambda_{i}}{\lambda_{i}-\lambda_{j}+L_{i}^{0}-L_{j}^{0}} \quad, \quad \text { where } 1 \leq r \leq j \text {, }
\end{aligned}
$$

and

$$
C_{i}^{i}=s_{i}^{0}(0)-\sum_{r=0}^{j} C_{i}^{r}
$$




\section{APPENDIX C:}

\section{TABULAR RESULTS REQUESTED IN THE SCENARIO*}

* The maximum value in each column of each table is shown in bold type. Capital letters at the beginning of each table title are requested format for results files, as specified in Section A.13 of Appendix A of this report (pages 49 and 50). 


\section{APPENDIX C:}

\section{TABULAR RESULTS REQUESTED IN THE SCENARIO*}

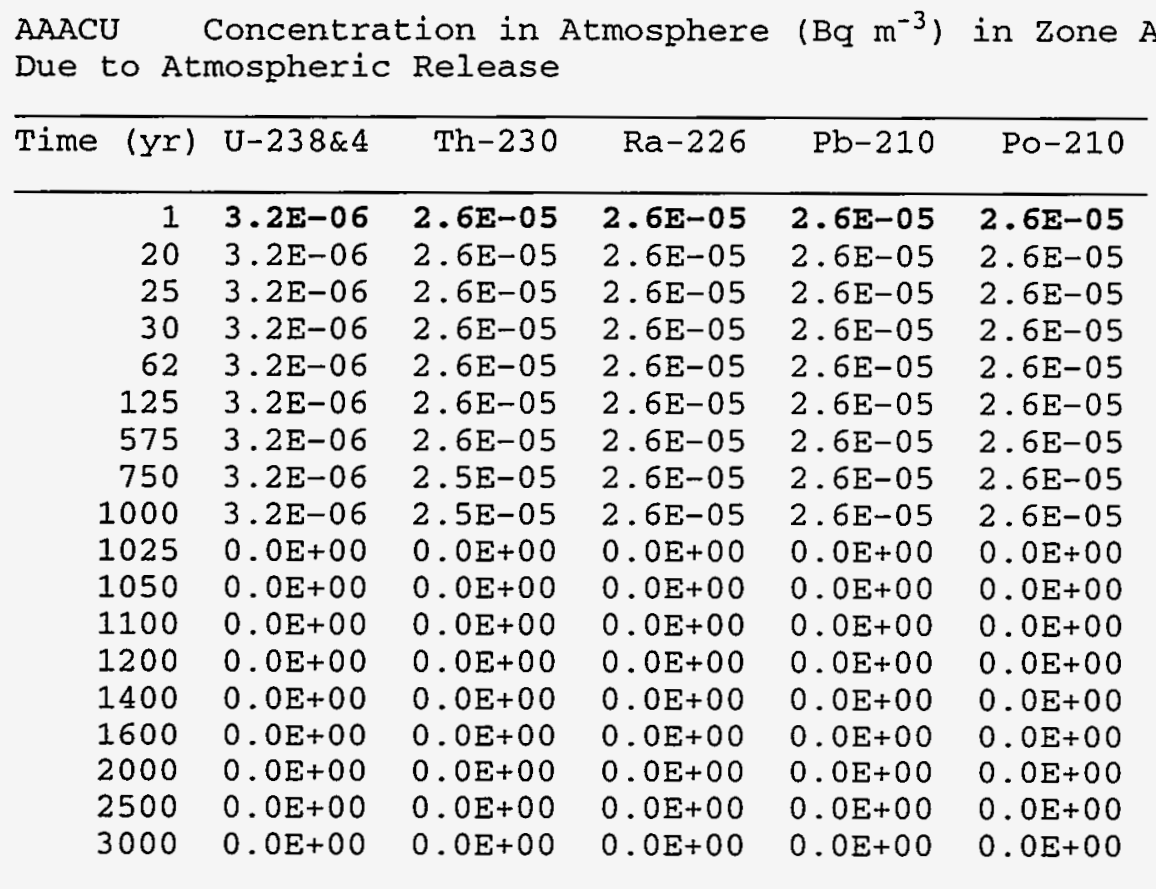

AAADU Inhalation Dose in Zone A Due to Atmospheric Release (SV $\mathrm{Yr}^{-1}$ )

\begin{tabular}{rcccccc}
\hline Time $(y r)$ & $\mathrm{U}-238 \& 4$ & Th-230 & Ra-226 & Pb-210 & Po-210 & Chain \\
\hline 1 & $6.2 \mathrm{E}-07$ & $\mathbf{7 . 7 \mathrm { E } - 0 6}$ & $\mathbf{3 . 2 \mathrm { E } - 0 7}$ & $\mathbf{3 . 3 \mathrm { E } - 0 7}$ & $\mathbf{2 . 9 \mathrm { E } - 0 7}$ & $9.2 \mathrm{E}-06$ \\
20 & $6.2 \mathrm{E}-07$ & $7.7 \mathrm{E}-06$ & $3.2 \mathrm{E}-07$ & $3.3 \mathrm{E}-07$ & $2.9 \mathrm{E}-07$ & $9.2 \mathrm{E}-06$ \\
25 & $6.2 \mathrm{E}-07$ & $7.7 \mathrm{E}-06$ & $3.2 \mathrm{E}-07$ & $3.3 \mathrm{E}-07$ & $2.9 \mathrm{E}-07$ & $9.2 \mathrm{E}-06$ \\
30 & $6.2 \mathrm{E}-07$ & $7.7 \mathrm{E}-06$ & $3.2 \mathrm{E}-07$ & $3.3 \mathrm{E}-07$ & $2.9 \mathrm{E}-07$ & $9.2 \mathrm{E}-06$ \\
62 & $6.2 \mathrm{E}-07$ & $7.7 \mathrm{E}-06$ & $3.2 \mathrm{E}-07$ & $3.3 \mathrm{E}-07$ & $2.9 \mathrm{E}-07$ & $9.2 \mathrm{E}-06$ \\
125 & $6.2 \mathrm{E}-07$ & $7.7 \mathrm{E}-06$ & $3.2 \mathrm{E}-07$ & $3.3 \mathrm{E}-07$ & $2.9 \mathrm{E}-07$ & $9.2 \mathrm{E}-06$ \\
575 & $6.2 \mathrm{E}-07$ & $7.6 \mathrm{E}-06$ & $3.2 \mathrm{E}-07$ & $3.3 \mathrm{E}-07$ & $2.9 \mathrm{E}-07$ & $9.2 \mathrm{E}-06$ \\
750 & $6.2 \mathrm{E}-07$ & $7.6 \mathrm{E}-06$ & $3.2 \mathrm{E}-07$ & $3.3 \mathrm{E}-07$ & $2.9 \mathrm{E}-07$ & $9.2 \mathrm{E}-06$ \\
1000 & $6.2 \mathrm{E}-07$ & $7.6 \mathrm{E}-06$ & $3.2 \mathrm{E}-07$ & $3.3 \mathrm{E}-07$ & $2.9 \mathrm{E}-07$ & $9.2 \mathrm{E}-06$ \\
1025 & $0.0 \mathrm{E}+00$ & $0.0 \mathrm{E}+00$ & $0.0 \mathrm{E}+00$ & $0.0 \mathrm{E}+00$ & $0.0 \mathrm{E}+00$ & $0.0 \mathrm{E}+00$ \\
1050 & $0.0 \mathrm{E}+00$ & $0.0 \mathrm{E}+00$ & $0.0 \mathrm{E}+00$ & $0.0 \mathrm{E}+00$ & $0.0 \mathrm{E}+00$ & $0.0 \mathrm{E}+00$ \\
1100 & $0.0 \mathrm{E}+00$ & $0.0 \mathrm{E}+00$ & $0.0 \mathrm{E}+00$ & $0.0 \mathrm{E}+00$ & $0.0 \mathrm{E}+00$ & $0.0 \mathrm{E}+00$ \\
1200 & $0.0 \mathrm{E}+00$ & $0.0 \mathrm{E}+00$ & $0.0 \mathrm{E}+00$ & $0.0 \mathrm{E}+00$ & $0.0 \mathrm{E}+00$ & $0.0 \mathrm{E}+00$ \\
1400 & $0.0 \mathrm{E}+00$ & $0.0 \mathrm{E}+00$ & $0.0 \mathrm{E}+00$ & $0.0 \mathrm{E}+00$ & $0.0 \mathrm{E}+00$ & $0.0 \mathrm{E}+00$ \\
1600 & $0.0 \mathrm{E}+00$ & $0.0 \mathrm{E}+00$ & $0.0 \mathrm{E}+00$ & $0.0 \mathrm{E}+00$ & $0.0 \mathrm{E}+00$ & $0.0 \mathrm{E}+00$ \\
2000 & $0.0 \mathrm{E}+00$ & $0.0 \mathrm{E}+00$ & $0.0 \mathrm{E}+00$ & $0.0 \mathrm{E}+00$ & $0.0 \mathrm{E}+00$ & $0.0 \mathrm{E}+00$ \\
2500 & $0.0 \mathrm{E}+00$ & $0.0 \mathrm{E}+00$ & $0.0 \mathrm{E}+00$ & $0.0 \mathrm{E}+00$ & $0.0 \mathrm{E}+00$ & $0.0 \mathrm{E}+00$ \\
3000 & $0.0 \mathrm{E}+00$ & $0.0 \mathrm{E}+00$ & $0.0 \mathrm{E}+00$ & $0.0 \mathrm{E}+00$ & $0.0 \mathrm{E}+00$ & $0.0 \mathrm{E}+00$
\end{tabular}

The maximum value in each column of each table is shown in bold type. Capital letters at the beginning of each table title are requested format for results files, as specified in Section A.13 of Appendix A of this report (pages 49 and 50). 
AAARU Inhalation Cancer Risk in Zone A Due to Atmospheric Release

\begin{tabular}{rrrrrrr}
\hline Time (Yr) & $\mathrm{U}-238 \& 4$ & Th-230 & Ra-226 & Pb-210 & Po-210 & Chain \\
\hline 1 & $1.4 \mathrm{E}-06$ & $8.2 \mathrm{E}-06$ & $8.5 \mathrm{E}-07$ & $7.4 \mathrm{E}-07$ & $3.7 \mathrm{E}-07$ & $1.2 \mathrm{E}-05$ \\
25 & $1.4 \mathrm{E}-06$ & $8.2 \mathrm{E}-06$ & $8.5 \mathrm{E}-07$ & $7.4 \mathrm{E}-07$ & $3.7 \mathrm{E}-07$ & $1.2 \mathrm{E}-05$ \\
30 & $1.4 \mathrm{E}-06$ & $8.2 \mathrm{E}-06$ & $8.5 \mathrm{E}-07$ & $7.4 \mathrm{E}-07$ & $3.7 \mathrm{E}-07$ & $1.2 \mathrm{E}-05$ \\
62 & $1.4 \mathrm{E}-06$ & $8.2 \mathrm{E}-06$ & $8.5 \mathrm{E}-07$ & $7.4 \mathrm{E}-07$ & $3.7 \mathrm{E}-07$ & $1.2 \mathrm{E}-05$ \\
125 & $1.4 \mathrm{E}-06$ & $8.2 \mathrm{E}-06$ & $8.5 \mathrm{E}-07$ & $7.4 \mathrm{E}-07$ & $3.7 \mathrm{E}-07$ & $1.2 \mathrm{E}-05$ \\
575 & $1.4 \mathrm{E}-06$ & $8.2 \mathrm{E}-06$ & $8.5 \mathrm{E}-07$ & $7.4 \mathrm{E}-07$ & $3.7 \mathrm{E}-07$ & $1.2 \mathrm{E}-05$ \\
750 & $1.4 \mathrm{E}-06$ & $8.2 \mathrm{E}-06$ & $8.5 \mathrm{E}-07$ & $7.4 \mathrm{E}-07$ & $3.7 \mathrm{E}-07$ & $1.1 \mathrm{E}-05$ \\
1000 & $1.4 \mathrm{E}-06$ & $8.2 \mathrm{E}-06$ & $8.5 \mathrm{E}-07$ & $7.4 \mathrm{E}-07$ & $3.7 \mathrm{E}-07$ & $1.1 \mathrm{E}-05$ \\
1025 & $0.0 \mathrm{E}+00$ & $0.0 \mathrm{E}+00$ & $0.0 \mathrm{E}+00$ & $0.0 \mathrm{E}+00$ & $0.0 \mathrm{E}+00$ & $0.0 \mathrm{E}+00$ \\
1050 & $0.0 \mathrm{E}+00$ & $0.0 \mathrm{E}+00$ & $0.0 \mathrm{E}+00$ & $0.0 \mathrm{E}+00$ & $0.0 \mathrm{E}+00$ & $0.0 \mathrm{E}+00$ \\
1100 & $0.0 \mathrm{E}+00$ & $0.0 \mathrm{E}+00$ & $0.0 \mathrm{E}+00$ & $0.0 \mathrm{E}+00$ & $0.0 \mathrm{E}+00$ & $0.0 \mathrm{E}+00$ \\
1200 & $0.0 \mathrm{E}+00$ & $0.0 \mathrm{E}+00$ & $0.0 \mathrm{E}+00$ & $0.0 \mathrm{E}+00$ & $0.0 \mathrm{E}+00$ & $0.0 \mathrm{E}+00$ \\
1400 & $0.0 \mathrm{E}+00$ & $0.0 \mathrm{E}+00$ & $0.0 \mathrm{E}+00$ & $0.0 \mathrm{E}+00$ & $0.0 \mathrm{E}+00$ & $0.0 \mathrm{E}+00$ \\
1600 & $0.0 \mathrm{E}+00$ & $0.0 \mathrm{E}+00$ & $0.0 \mathrm{E}+00$ & $0.0 \mathrm{E}+00$ & $0.0 \mathrm{E}+00$ & $0.0 \mathrm{E}+00$ \\
2000 & $0.0 \mathrm{E}+00$ & $0.0 \mathrm{E}+00$ & $0.0 \mathrm{E}+00$ & $0.0 \mathrm{E}+00$ & $0.0 \mathrm{E}+00$ & $0.0 \mathrm{E}+00$ \\
2500 & $0.0 \mathrm{E}+00$ & $0.0 \mathrm{E}+00$ & $0.0 \mathrm{E}+00$ & $0.0 \mathrm{E}+00$ & $0.0 \mathrm{E}+00$ & $0.0 \mathrm{E}+00$ \\
3000 & $0.0 \mathrm{E}+00$ & $0.0 \mathrm{E}+00$ & $0.0 \mathrm{E}+00$ & $0.0 \mathrm{E}+00$ & $0.0 \mathrm{E}+00$ & $0.0 \mathrm{E}+00$ \\
\end{tabular}

AAACM Concentration in Atmosphere $\left(\mathrm{mg} \mathrm{m}^{-3}\right.$ ) in Zone $\mathrm{A}$ Due to Atmospheric Release

\begin{tabular}{rrrr}
\hline Time $(y r)$ & As & $\mathrm{Cr}$ & $\mathrm{Ni}$ \\
\hline 1 & $1.8 \mathrm{E}-07$ & $1.8 \mathrm{E}-08$ & $1.8 \mathrm{E}-09$ \\
20 & $1.8 \mathrm{E}-07$ & $1.8 \mathrm{E}-08$ & $1.8 \mathrm{E}-09$ \\
25 & $1.8 \mathrm{E}-07$ & $1.8 \mathrm{E}-08$ & $1.8 \mathrm{E}-09$ \\
30 & $1.8 \mathrm{E}-07$ & $1.8 \mathrm{E}-08$ & $1.8 \mathrm{E}-09$ \\
62 & $1.8 \mathrm{E}-07$ & $1.8 \mathrm{E}-08$ & $1.8 \mathrm{E}-09$ \\
125 & $1.8 \mathrm{E}-07$ & $1.8 \mathrm{E}-08$ & $1.8 \mathrm{E}-09$ \\
575 & $1.8 \mathrm{E}-07$ & $1.8 \mathrm{E}-08$ & $1.8 \mathrm{E}-09$ \\
750 & $1.8 \mathrm{E}-07$ & $1.8 \mathrm{E}-08$ & $1.8 \mathrm{E}-09$ \\
1000 & $1.8 \mathrm{E}-07$ & $1.8 \mathrm{E}-08$ & $1.8 \mathrm{E}-09$ \\
1025 & $0.0 \mathrm{E}+00$ & $0.0 \mathrm{E}+00$ & $0.0 \mathrm{E}+00$ \\
1050 & $0.0 \mathrm{E}+00$ & $0.0 \mathrm{E}+00$ & $0.0 \mathrm{E}+00$ \\
1100 & $0.0 \mathrm{E}+00$ & $0.0 \mathrm{E}+00$ & $0.0 \mathrm{E}+00$ \\
1200 & $0.0 \mathrm{E}+00$ & $0.0 \mathrm{E}+00$ & $0.0 \mathrm{E}+00$ \\
1400 & $0.0 \mathrm{E}+00$ & $0.0 \mathrm{E}+00$ & $0.0 \mathrm{E}+00$ \\
1600 & $0.0 \mathrm{E}+00$ & $0.0 \mathrm{E}+00$ & $0.0 \mathrm{E}+00$ \\
2000 & $0.0 \mathrm{E}+00$ & $0.0 \mathrm{E}+00$ & $0.0 \mathrm{E}+00$ \\
2500 & $0.0 \mathrm{E}+00$ & $0.0 \mathrm{E}+00$ & $0.0 \mathrm{E}+00$ \\
3000 & $0.0 \mathrm{E}+00$ & $0.0 \mathrm{E}+00$ & $0.0 \mathrm{E}+00$ \\
& & & \\
\hline
\end{tabular}

AAAIM Inhalation Intake in Zone A Due to Atmospheric Release ( $\mathrm{mg} \mathrm{Yr}^{-1}$ )

\begin{tabular}{rrrr}
\hline Time $(Y Y)$ & As & $\mathrm{Cr}$ & $\mathrm{Ni}$ \\
\hline 1 & $1.1 \mathrm{E}-03$ & $1.1 \mathrm{E}-04$ & $1.1 \mathrm{E}-05$ \\
20 & $1.1 \mathrm{E}-03$ & $1.1 \mathrm{E}-04$ & $1.1 \mathrm{E}-05$ \\
25 & $1.1 \mathrm{E}-03$ & $1.1 \mathrm{E}-04$ & $1.1 \mathrm{E}-05$ \\
30 & $1.1 \mathrm{E}-03$ & $1.1 \mathrm{E}-04$ & $1.1 \mathrm{E}-05$ \\
62 & $1.1 \mathrm{E}-03$ & $1.1 \mathrm{E}-04$ & $1.1 \mathrm{E}-05$ \\
125 & $1.1 \mathrm{E}-03$ & $1.1 \mathrm{E}-04$ & $1.1 \mathrm{E}-05$ \\
575 & $1.1 \mathrm{E}-03$ & $1.1 \mathrm{E}-04$ & $1.1 \mathrm{E}-05$ \\
750 & $1.1 \mathrm{E}-03$ & $1.1 \mathrm{E}-04$ & $1.1 \mathrm{E}-05$ \\
1000 & $1.1 \mathrm{E}-03$ & $1.1 \mathrm{E}-04$ & $1.1 \mathrm{E}-05$ \\
1025 & $0.0 \mathrm{E}+00$ & $0.0 \mathrm{E}+00$ & $0.0 \mathrm{E}+00$ \\
1050 & $0.0 \mathrm{E}+00$ & $0.0 \mathrm{E}+00$ & $0.0 \mathrm{E}+00$ \\
1100 & $0.0 \mathrm{E}+00$ & $0.0 \mathrm{E}+00$ & $0.0 \mathrm{E}+00$ \\
1200 & $0.0 \mathrm{E}+00$ & $0.0 \mathrm{E}+00$ & $0.0 \mathrm{E}+00$ \\
1400 & $0.0 \mathrm{E}+00$ & $0.0 \mathrm{E}+00$ & $0.0 \mathrm{E}+00$ \\
1600 & $0.0 \mathrm{E}+00$ & $0.0 \mathrm{E}+00$ & $0.0 \mathrm{E}+00$ \\
2000 & $0.0 \mathrm{E}+00$ & $0.0 \mathrm{E}+00$ & $0.0 \mathrm{E}+00$ \\
2500 & $0.0 \mathrm{E}+00$ & $0.0 \mathrm{E}+00$ & $0.0 \mathrm{E}+00$ \\
3000 & $0.0 \mathrm{E}+00$ & $0.0 \mathrm{E}+00$ & $0.0 \mathrm{E}+00$
\end{tabular}


AAARM Inhalation Cancer Risk in Zone A Due to Atmospheric Release

\begin{tabular}{rrrr}
\hline Time $(\mathrm{Yr})$ & As & $\mathrm{Cr}$ & $\mathrm{Ni}$ \\
\hline 1 & $5.1 \mathrm{E}-04$ & $4.2 \mathrm{E}-05$ & $9.0 \mathrm{E}-08$ \\
20 & $5.1 \mathrm{E}-04$ & $4.2 \mathrm{E}-05$ & $9.0 \mathrm{E}-08$ \\
25 & $5.1 \mathrm{E}-04$ & $4.2 \mathrm{E}-05$ & $9.0 \mathrm{E}-08$ \\
30 & $5.1 \mathrm{E}-04$ & $4.2 \mathrm{E}-05$ & $9.0 \mathrm{E}-08$ \\
62 & $5.1 \mathrm{E}-04$ & $4.2 \mathrm{E}-05$ & $9.0 \mathrm{E}-08$ \\
125 & $5.1 \mathrm{E}-04$ & $4.2 \mathrm{E}-05$ & $9.0 \mathrm{E}-08$ \\
575 & $5.1 \mathrm{E}-04$ & $4.2 \mathrm{E}-05$ & $9.0 \mathrm{E}-08$ \\
750 & $5.1 \mathrm{E}-04$ & $4.2 \mathrm{E}-05$ & $9.0 \mathrm{E}-08$ \\
1000 & $5.1 \mathrm{E}-04$ & $4.2 \mathrm{E}-05$ & $9.0 \mathrm{E}-08$ \\
1025 & $0.0 \mathrm{E}+00$ & $0.0 \mathrm{E}+00$ & $0.0 \mathrm{E}+00$ \\
1050 & $0.0 \mathrm{E}+00$ & $0.0 \mathrm{E}+00$ & $0.0 \mathrm{E}+00$ \\
1100 & $0.0 \mathrm{E}+00$ & $0.0 \mathrm{E}+00$ & $0.0 \mathrm{E}+00$ \\
1200 & $0.0 \mathrm{E}+00$ & $0.0 \mathrm{E}+00$ & $0.0 \mathrm{E}+00$ \\
1400 & $0.0 \mathrm{E}+00$ & $0.0 \mathrm{E}+00$ & $0.0 \mathrm{E}+00$ \\
1600 & $0.0 \mathrm{E}+00$ & $0.0 \mathrm{E}+00$ & $0.0 \mathrm{E}+00$ \\
2000 & $0.0 \mathrm{E}+00$ & $0.0 \mathrm{E}+00$ & $0.0 \mathrm{E}+00$ \\
2500 & $0.0 \mathrm{E}+00$ & $0.0 \mathrm{E}+00$ & $0.0 \mathrm{E}+00$ \\
3000 & $0.0 \mathrm{E}+00$ & $0.0 \mathrm{E}+00$ & $0.0 \mathrm{E}+00$
\end{tabular}

ABACU Concentration in Atmosphere $\left(\mathrm{Bq} \mathrm{m} \mathrm{m}^{-3}\right)$ in Zone $B$ Due to Atmospheric Release

\begin{tabular}{rccccc}
\hline Time $(\mathrm{yr})$ & $\mathrm{U}-238 \& 4$ & Th-230 & Ra-226 & $\mathrm{Pb}-210$ & PO-210 \\
\hline 1 & $5.8 \mathrm{E}-07$ & $\mathbf{4 . 6 \mathrm { E } - 0 6}$ & $\mathbf{4 . 6 \mathrm { E } - 0 6}$ & $\mathbf{4 . 6 \mathrm { E } - 0 6}$ & $\mathbf{4 . 6 \mathrm { E } - 0 6}$ \\
20 & $5.8 \mathrm{E}-07$ & $4.6 \mathrm{E}-06$ & $4.6 \mathrm{E}-06$ & $4.6 \mathrm{E}-06$ & $4.6 \mathrm{E}-06$ \\
25 & $5.8 \mathrm{E}-07$ & $4.6 \mathrm{E}-06$ & $4.6 \mathrm{E}-06$ & $4.6 \mathrm{E}-06$ & $4.6 \mathrm{E}-06$ \\
30 & $5.8 \mathrm{E}-07$ & $4.6 \mathrm{E}-06$ & $4.6 \mathrm{E}-06$ & $4.6 \mathrm{E}-06$ & $4.6 \mathrm{E}-06$ \\
62 & $5.8 \mathrm{E}-07$ & $4.6 \mathrm{E}-06$ & $4.6 \mathrm{E}-06$ & $4.6 \mathrm{E}-06$ & $4.6 \mathrm{E}-06$ \\
125 & $5.8 \mathrm{E}-07$ & $4.6 \mathrm{E}-06$ & $4.6 \mathrm{E}-06$ & $4.6 \mathrm{E}-06$ & $4.6 \mathrm{E}-06$ \\
575 & $5.7 \mathrm{E}-07$ & $4.6 \mathrm{E}-06$ & $4.6 \mathrm{E}-06$ & $4.6 \mathrm{E}-06$ & $4.6 \mathrm{E}-06$ \\
750 & $5.7 \mathrm{E}-07$ & $4.6 \mathrm{E}-06$ & $4.6 \mathrm{E}-06$ & $4.6 \mathrm{E}-06$ & $4.6 \mathrm{E}-06$ \\
1000 & $5.7 \mathrm{E}-07$ & $4.6 \mathrm{E}-06$ & $4.6 \mathrm{E}-06$ & $4.6 \mathrm{E}-06$ & $4.6 \mathrm{E}-06$ \\
1025 & $0.0 \mathrm{E}+00$ & $0.0 \mathrm{E}+00$ & $0.0 \mathrm{E}+00$ & $0.0 \mathrm{E}+00$ & $0.0 \mathrm{E}+00$ \\
1050 & $0.0 \mathrm{E}+00$ & $0.0 \mathrm{E}+00$ & $0.0 \mathrm{E}+00$ & $0.0 \mathrm{E}+00$ & $0.0 \mathrm{E}+00$ \\
1100 & $0.0 \mathrm{E}+00$ & $0.0 \mathrm{E}+00$ & $0.0 \mathrm{E}+00$ & $0.0 \mathrm{E}+00$ & $0.0 \mathrm{E}+00$ \\
1200 & $0.0 \mathrm{E}+00$ & $0.0 \mathrm{E}+00$ & $0.0 \mathrm{E}+00$ & $0.0 \mathrm{E}+00$ & $0.0 \mathrm{E}+00$ \\
1400 & $0.0 \mathrm{E}+00$ & $0.0 \mathrm{E}+00$ & $0.0 \mathrm{E}+00$ & $0.0 \mathrm{E}+00$ & $0.0 \mathrm{E}+00$ \\
1600 & $0.0 \mathrm{E}+00$ & $0.0 \mathrm{E}+00$ & $0.0 \mathrm{E}+00$ & $0.0 \mathrm{E}+00$ & $0.0 \mathrm{E}+00$ \\
2000 & $0.0 \mathrm{E}+00$ & $0.0 \mathrm{E}+00$ & $0.0 \mathrm{E}+00$ & $0.0 \mathrm{E}+00$ & $0.0 \mathrm{E}+00$ \\
2500 & $0.0 \mathrm{E}+00$ & $0.0 \mathrm{E}+00$ & $0.0 \mathrm{E}+00$ & $0.0 \mathrm{E}+00$ & $0.0 \mathrm{E}+00$ \\
3000 & $0.0 \mathrm{E}+00$ & $0.0 \mathrm{E}+00$ & $0.0 \mathrm{E}+00$ & $0.0 \mathrm{E}+00$ & $0.0 \mathrm{E}+00$ \\
\hline
\end{tabular}


ABADU Inhalation Dose in Zone B Due to Atmospheric Release
(Sv $\mathrm{Yr}^{-1}$ )

\begin{tabular}{rrrrrrr}
\hline Time (yI) & $\mathrm{U}-238 \& 4$ & $\mathrm{Th}-230$ & $\mathrm{Ra}-226$ & $\mathrm{~Pb}-210$ & Po-210 & Chain \\
\hline 1 & $1.1 \mathrm{E}-07$ & $1.4 \mathrm{E}-06$ & $5.7 \mathrm{E}-08$ & $6.0 \mathrm{E}-08$ & $5.2 \mathrm{E}-08$ & $1.7 \mathrm{E}-06$ \\
20 & $1.1 \mathrm{E}-07$ & $1.4 \mathrm{E}-06$ & $5.7 \mathrm{E}-08$ & $6.0 \mathrm{E}-08$ & $5.2 \mathrm{E}-08$ & $1.7 \mathrm{E}-06$ \\
25 & $1.1 \mathrm{E}-07$ & $1.4 \mathrm{E}-06$ & $5.7 \mathrm{E}-08$ & $6.0 \mathrm{E}-08$ & $5.2 \mathrm{E}-08$ & $1.7 \mathrm{E}-06$ \\
30 & $1.1 \mathrm{E}-07$ & $1.4 \mathrm{E}-06$ & $5.7 \mathrm{E}-08$ & $6.0 \mathrm{E}-08$ & $5.2 \mathrm{E}-08$ & $1.7 \mathrm{E}-06$ \\
62 & $1.1 \mathrm{E}-07$ & $1.4 \mathrm{E}-06$ & $5.7 \mathrm{E}-08$ & $6.0 \mathrm{E}-08$ & $5.2 \mathrm{E}-08$ & $1.7 \mathrm{E}-06$ \\
125 & $1.1 \mathrm{E}-07$ & $1.4 \mathrm{E}-06$ & $5.7 \mathrm{E}-08$ & $6.0 \mathrm{E}-08$ & $5.2 \mathrm{E}-08$ & $1.7 \mathrm{E}-06$ \\
575 & $1.1 \mathrm{E}-07$ & $1.4 \mathrm{E}-06$ & $5.7 \mathrm{E}-08$ & $6.0 \mathrm{E}-08$ & $5.2 \mathrm{E}-08$ & $1.7 \mathrm{E}-06$ \\
750 & $1.1 \mathrm{E}-07$ & $1.4 \mathrm{E}-06$ & $5.7 \mathrm{E}-08$ & $6.0 \mathrm{E}-08$ & $5.2 \mathrm{E}-08$ & $1.7 \mathrm{E}-06$ \\
1000 & $1.1 \mathrm{E}-07$ & $1.4 \mathrm{E}-06$ & $5.7 \mathrm{E}-08$ & $6.0 \mathrm{E}-08$ & $5.2 \mathrm{E}-08$ & $1.7 \mathrm{E}-06$ \\
1025 & $0.0 \mathrm{E}+00$ & $0.0 \mathrm{E}+00$ & $0.0 \mathrm{E}+00$ & $0.0 \mathrm{E}+00$ & $0.0 \mathrm{E}+00$ & $0.0 \mathrm{E}+00$ \\
1050 & $0.0 \mathrm{E}+00$ & $0.0 \mathrm{E}+00$ & $0.0 \mathrm{E}+00$ & $0.0 \mathrm{E}+00$ & $0.0 \mathrm{E}+00$ & $0.0 \mathrm{E}+00$ \\
1100 & $0.0 \mathrm{E}+00$ & $0.0 \mathrm{E}+00$ & $0.0 \mathrm{E}+00$ & $0.0 \mathrm{E}+00$ & $0.0 \mathrm{E}+00$ & $0.0 \mathrm{E}+00$ \\
1200 & $0.0 \mathrm{E}+00$ & $0.0 \mathrm{E}+00$ & $0.0 \mathrm{E}+00$ & $0.0 \mathrm{E}+00$ & $0.0 \mathrm{E}+00$ & $0.0 \mathrm{E}+00$ \\
1400 & $0.0 \mathrm{E}+00$ & $0.0 \mathrm{E}+00$ & $0.0 \mathrm{E}+00$ & $0.0 \mathrm{E}+00$ & $0.0 \mathrm{E}+00$ & $0.0 \mathrm{E}+00$ \\
1600 & $0.0 \mathrm{E}+00$ & $0.0 \mathrm{E}+00$ & $0.0 \mathrm{E}+00$ & $0.0 \mathrm{E}+00$ & $0.0 \mathrm{E}+00$ & $0.0 \mathrm{E}+00$ \\
2000 & $0.0 \mathrm{E}+00$ & $0.0 \mathrm{E}+00$ & $0.0 \mathrm{E}+00$ & $0.0 \mathrm{E}+00$ & $0.0 \mathrm{E}+00$ & $0.0 \mathrm{E}+00$ \\
2500 & $0.0 \mathrm{E}+00$ & $0.0 \mathrm{E}+00$ & $0.0 \mathrm{E}+00$ & $0.0 \mathrm{E}+00$ & $0.0 \mathrm{E}+00$ & $0.0 \mathrm{E}+00$ \\
3000 & $0.0 \mathrm{E}+00$ & $0.0 \mathrm{E}+00$ & $0.0 \mathrm{E}+00$ & $0.0 \mathrm{E}+00$ & $0.0 \mathrm{E}+00$ & $0.0 \mathrm{E}+00$
\end{tabular}

ABARU Inhalation Cancer Risk in Zone B Due to Atmospheric Release

\begin{tabular}{rrrrrrr}
\hline Time $(Y \Upsilon)$ & $U-238 \& 4$ & $T h-230$ & Ra-226 & Pb-210 & Po-210 & Chain \\
\hline 1 & $2.5 \mathrm{E}-07$ & $1.5 \mathrm{E}-06$ & $1.5 \mathrm{E}-07$ & $1.3 \mathrm{E}-07$ & $6.7 \mathrm{E}-08$ & $2.1 \mathrm{E}-06$ \\
20 & $2.5 \mathrm{E}-07$ & $1.5 \mathrm{E}-06$ & $1.5 \mathrm{E}-07$ & $1.3 \mathrm{E}-07$ & $6.7 \mathrm{E}-08$ & $2.1 \mathrm{E}-06$ \\
25 & $2.5 \mathrm{E}-07$ & $1.5 \mathrm{E}-06$ & $1.5 \mathrm{E}-07$ & $1.3 \mathrm{E}-07$ & $6.7 \mathrm{E}-08$ & $2.1 \mathrm{E}-06$ \\
30 & $2.5 \mathrm{E}-07$ & $1.5 \mathrm{E}-06$ & $1.5 \mathrm{E}-07$ & $1.3 \mathrm{E}-07$ & $6.7 \mathrm{E}-08$ & $2.1 \mathrm{E}-06$ \\
62 & $2.5 \mathrm{E}-07$ & $1.5 \mathrm{E}-06$ & $1.5 \mathrm{E}-07$ & $1.3 \mathrm{E}-07$ & $6.7 \mathrm{E}-08$ & $2.1 \mathrm{E}-06$ \\
125 & $2.5 \mathrm{E}-07$ & $1.5 \mathrm{E}-06$ & $1.5 \mathrm{E}-07$ & $1.3 \mathrm{E}-07$ & $6.7 \mathrm{E}-08$ & $2.1 \mathrm{E}-06$ \\
575 & $2.5 \mathrm{E}-07$ & $1.5 \mathrm{E}-06$ & $1.5 \mathrm{E}-07$ & $1.3 \mathrm{E}-07$ & $6.7 \mathrm{E}-08$ & $2.1 \mathrm{E}-06$ \\
750 & $2.5 \mathrm{E}-07$ & $1.5 \mathrm{E}-06$ & $1.5 \mathrm{E}-07$ & $1.3 \mathrm{E}-07$ & $6.7 \mathrm{E}-08$ & $2.1 \mathrm{E}-06$ \\
1000 & $2.5 \mathrm{E}-07$ & $1.5 \mathrm{E}-06$ & $1.5 \mathrm{E}-07$ & $1.3 \mathrm{E}-07$ & $6.7 \mathrm{E}-08$ & $2.1 \mathrm{E}-06$ \\
1025 & $0.0 \mathrm{E}+00$ & $0.0 \mathrm{E}+00$ & $0.0 \mathrm{E}+00$ & $0.0 \mathrm{E}+00$ & $0.0 \mathrm{E}+00$ & $0.0 \mathrm{E}+00$ \\
1050 & $0.0 \mathrm{E}+00$ & $0.0 \mathrm{E}+00$ & $0.0 \mathrm{E}+00$ & $0.0 \mathrm{E}+00$ & $0.0 \mathrm{E}+00$ & $0.0 \mathrm{E}+00$ \\
1100 & $0.0 \mathrm{E}+00$ & $0.0 \mathrm{E}+00$ & $0.0 \mathrm{E}+00$ & $0.0 \mathrm{E}+00$ & $0.0 \mathrm{E}+00$ & $0.0 \mathrm{E}+00$ \\
1200 & $0.0 \mathrm{E}+00$ & $0.0 \mathrm{E}+00$ & $0.0 \mathrm{E}+00$ & $0.0 \mathrm{E}+00$ & $0.0 \mathrm{E}+00$ & $0.0 \mathrm{E}+00$ \\
1400 & $0.0 \mathrm{E}+00$ & $0.0 \mathrm{E}+00$ & $0.0 \mathrm{E}+00$ & $0.0 \mathrm{E}+00$ & $0.0 \mathrm{E}+00$ & $0.0 \mathrm{E}+00$ \\
1600 & $0.0 \mathrm{E}+00$ & $0.0 \mathrm{E}+00$ & $0.0 \mathrm{E}+00$ & $0.0 \mathrm{E}+00$ & $0.0 \mathrm{E}+00$ & $0.0 \mathrm{E}+00$ \\
2000 & $0.0 \mathrm{E}+00$ & $0.0 \mathrm{E}+00$ & $0.0 \mathrm{E}+00$ & $0.0 \mathrm{E}+00$ & $0.0 \mathrm{E}+00$ & $0.0 \mathrm{E}+00$ \\
2500 & $0.0 \mathrm{E}+00$ & $0.0 \mathrm{E}+00$ & $0.0 \mathrm{E}+00$ & $0.0 \mathrm{E}+00$ & $0.0 \mathrm{E}+00$ & $0.0 \mathrm{E}+00$ \\
3000 & $0.0 \mathrm{E}+00$ & $0.0 \mathrm{E}+00$ & $0.0 \mathrm{E}+00$ & $0.0 \mathrm{E}+00$ & $0.0 \mathrm{E}+00$ & $0.0 \mathrm{E}+00$
\end{tabular}


ABACM Concentration in Atmosphere $\left(\mathrm{mg} \mathrm{m}^{-3}\right)$ in Zone $B$ Due to Atmospheric Release

\begin{tabular}{rrrr}
\hline Time $(y r)$ & As & $\mathrm{Cr}$ & $\mathrm{Ni}$ \\
\hline 1 & $3.3 \mathrm{E}-08$ & $3.3 \mathrm{E}-09$ & $3.3 \mathrm{E}-10$ \\
20 & $3.3 \mathrm{E}-08$ & $3.3 \mathrm{E}-09$ & $3.3 \mathrm{E}-10$ \\
25 & $3.3 \mathrm{E}-08$ & $3.3 \mathrm{E}-09$ & $3.3 \mathrm{E}-10$ \\
30 & $3.3 \mathrm{E}-08$ & $3.3 \mathrm{E}-09$ & $3.3 \mathrm{E}-10$ \\
62 & $3.3 \mathrm{E}-08$ & $3.3 \mathrm{E}-09$ & $3.3 \mathrm{E}-10$ \\
125 & $3.3 \mathrm{E}-08$ & $3.3 \mathrm{E}-09$ & $3.3 \mathrm{E}-10$ \\
575 & $3.3 \mathrm{E}-08$ & $3.3 \mathrm{E}-09$ & $3.3 \mathrm{E}-10$ \\
750 & $3.3 \mathrm{E}-08$ & $3.3 \mathrm{E}-09$ & $3.3 \mathrm{E}-10$ \\
1000 & $3.3 \mathrm{E}-08$ & $3.3 \mathrm{E}-09$ & $3.3 \mathrm{E}-10$ \\
1025 & $0.0 \mathrm{E}+00$ & $0.0 \mathrm{E}+00$ & $0.0 \mathrm{E}+00$ \\
1050 & $0.0 \mathrm{E}+00$ & $0.0 \mathrm{E}+00$ & $0.0 \mathrm{E}+00$ \\
1100 & $0.0 \mathrm{E}+00$ & $0.0 \mathrm{E}+00$ & $0.0 \mathrm{E}+00$ \\
1200 & $0.0 \mathrm{E}+00$ & $0.0 \mathrm{E}+00$ & $0.0 \mathrm{E}+00$ \\
1400 & $0.0 \mathrm{E}+00$ & $0.0 \mathrm{E}+00$ & $0.0 \mathrm{E}+00$ \\
1600 & $0.0 \mathrm{E}+00$ & $0.0 \mathrm{E}+00$ & $0.0 \mathrm{E}+00$ \\
2000 & $0.0 \mathrm{E}+00$ & $0.0 \mathrm{E}+00$ & $0.0 \mathrm{E}+00$ \\
2500 & $0.0 \mathrm{E}+00$ & $0.0 \mathrm{E}+00$ & $0.0 \mathrm{E}+00$ \\
3000 & $0.0 \mathrm{E}+00$ & $0.0 \mathrm{E}+00$ & $0.0 \mathrm{E}+00$ \\
\hline
\end{tabular}

ABARM Inhalation Cancer Risk in Zone B Due to Atmospheric Release

\begin{tabular}{rrrr}
\hline Time (yr) & As & $\mathrm{Cr}$ & $\mathrm{Ni}$ \\
\hline 1 & $9.3 \mathrm{E}-05$ & $7.6 \mathrm{E}-06$ & $1.6 \mathrm{E}-08$ \\
20 & $9.3 \mathrm{E}-05$ & $7.6 \mathrm{E}-06$ & $1.6 \mathrm{E}-08$ \\
25 & $9.3 \mathrm{E}-05$ & $7.6 \mathrm{E}-06$ & $1.6 \mathrm{E}-08$ \\
30 & $9.3 \mathrm{E}-05$ & $7.6 \mathrm{E}-06$ & $1.6 \mathrm{E}-08$ \\
62 & $9.3 \mathrm{E}-05$ & $7.6 \mathrm{E}-06$ & $1.6 \mathrm{E}-08$ \\
125 & $9.3 \mathrm{E}-05$ & $7.6 \mathrm{E}-06$ & $1.6 \mathrm{E}-08$ \\
575 & $9.3 \mathrm{E}-05$ & $7.6 \mathrm{E}-06$ & $1.6 \mathrm{E}-08$ \\
750 & $9.3 \mathrm{E}-05$ & $7.6 \mathrm{E}-06$ & $1.6 \mathrm{E}-08$ \\
1000 & $9.3 \mathrm{E}-05$ & $7.6 \mathrm{E}-06$ & $1.6 \mathrm{E}-08$ \\
1025 & $0.0 \mathrm{E}+00$ & $0.0 \mathrm{E}+00$ & $0.0 \mathrm{E}+00$ \\
1050 & $0.0 \mathrm{E}+00$ & $0.0 \mathrm{E}+00$ & $0.0 \mathrm{E}+00$ \\
1100 & $0.0 \mathrm{E}+00$ & $0.0 \mathrm{E}+00$ & $0.0 \mathrm{E}+00$ \\
1200 & $0.0 \mathrm{E}+00$ & $0.0 \mathrm{E}+00$ & $0.0 \mathrm{E}+00$ \\
1400 & $0.0 \mathrm{E}+00$ & $0.0 \mathrm{E}+00$ & $0.0 \mathrm{E}+00$ \\
1600 & $0.0 \mathrm{E}+00$ & $0.0 \mathrm{E}+00$ & $0.0 \mathrm{E}+00$ \\
2000 & $0.0 \mathrm{E}+00$ & $0.0 \mathrm{E}+00$ & $0.0 \mathrm{E}+00$ \\
2500 & $0.0 \mathrm{E}+00$ & $0.0 \mathrm{E}+00$ & $0.0 \mathrm{E}+00$ \\
3000 & $0.0 \mathrm{E}+00$ & $0.0 \mathrm{E}+00$ & $0.0 \mathrm{E}+00$ \\
& & & \\
\hline
\end{tabular}

ABAIM Inhalation Intake in Zone $B$ Due to Atmospheric Release ( $\mathrm{mg} \mathrm{yr}^{-1}$ )

\begin{tabular}{rrrr}
\hline Time $(y r)$ & As & $\mathrm{Cr}$ & $\mathrm{Ni}$ \\
\hline 1 & $1.9 \mathrm{E}-04$ & $1.9 \mathrm{E}-05$ & $1.9 \mathrm{E}-06$ \\
20 & $1.9 \mathrm{E}-04$ & $1.9 \mathrm{E}-05$ & $1.9 \mathrm{E}-06$ \\
25 & $1.9 \mathrm{E}-04$ & $1.9 \mathrm{E}-05$ & $1.9 \mathrm{E}-06$ \\
30 & $1.9 \mathrm{E}-04$ & $1.9 \mathrm{E}-05$ & $1.9 \mathrm{E}-06$ \\
62 & $1.9 \mathrm{E}-04$ & $1.9 \mathrm{E}-05$ & $1.9 \mathrm{E}-06$ \\
125 & $1.9 \mathrm{E}-04$ & $1.9 \mathrm{E}-05$ & $1.9 \mathrm{E}-06$ \\
575 & $1.9 \mathrm{E}-04$ & $1.9 \mathrm{E}-05$ & $1.9 \mathrm{E}-06$ \\
750 & $1.9 \mathrm{E}-04$ & $1.9 \mathrm{E}-05$ & $1.9 \mathrm{E}-06$ \\
1000 & $1.9 \mathrm{E}-04$ & $1.9 \mathrm{E}-05$ & $1.9 \mathrm{E}-06$ \\
1025 & $0.0 \mathrm{E}+00$ & $0.0 \mathrm{E}+00$ & $0.0 \mathrm{E}+00$ \\
1050 & $0.0 \mathrm{E}+00$ & $0.0 \mathrm{E}+00$ & $0.0 \mathrm{E}+00$ \\
1100 & $0.0 \mathrm{E}+00$ & $0.0 \mathrm{E}+00$ & $0.0 \mathrm{E}+00$ \\
1200 & $0.0 \mathrm{E}+00$ & $0.0 \mathrm{E}+00$ & $0.0 \mathrm{E}+00$ \\
1400 & $0.0 \mathrm{E}+00$ & $0.0 \mathrm{E}+00$ & $0.0 \mathrm{E}+00$ \\
1600 & $0.0 \mathrm{E}+00$ & $0.0 \mathrm{E}+00$ & $0.0 \mathrm{E}+00$ \\
2000 & $0.0 \mathrm{E}+00$ & $0.0 \mathrm{E}+00$ & $0.0 \mathrm{E}+00$ \\
2500 & $0.0 \mathrm{E}+00$ & $0.0 \mathrm{E}+00$ & $0.0 \mathrm{E}+00$ \\
3000 & $0.0 \mathrm{E}+00$ & $0.0 \mathrm{E}+00$ & $0.0 \mathrm{E}+00$
\end{tabular}


AABCU Concentration in Beef $\left(\mathrm{Bq} \mathrm{kg}^{-1}\right.$ ) in Zone $\mathrm{A}$ Due to Atmospheric Release

\begin{tabular}{rrrrrr}
\hline Time $(\mathrm{Yr})$ & $\mathrm{U}-238 \& 4$ & Th-230 & Ra-226 & Pb-210 & PO-210 \\
\hline 1 & $2.2 \mathrm{E}-02$ & $2.4 \mathrm{E}-03$ & $3.1 \mathrm{E}-03$ & $1.2 \mathrm{E}-02$ & $3.0 \mathrm{E}+00$ \\
20 & $2.4 \mathrm{E}-02$ & $2.5 \mathrm{E}-03$ & $3.7 \mathrm{E}-03$ & $1.3 \mathrm{E}-02$ & $3.2 \mathrm{E}+00$ \\
25 & $2.4 \mathrm{E}-02$ & $2.5 \mathrm{E}-03$ & $3.8 \mathrm{E}-03$ & $1.3 \mathrm{E}-02$ & $3.2 \mathrm{E}+00$ \\
30 & $2.4 \mathrm{E}-02$ & $2.5 \mathrm{E}-03$ & $4.0 \mathrm{E}-03$ & $1.3 \mathrm{E}-02$ & $3.3 \mathrm{E}+00$ \\
62 & $2.5 \mathrm{E}-02$ & $2.7 \mathrm{E}-03$ & $4.8 \mathrm{E}-03$ & $1.3 \mathrm{E}-02$ & $3.5 \mathrm{E}+00$ \\
125 & $2.7 \mathrm{E}-02$ & $2.8 \mathrm{E}-03$ & $5.9 \mathrm{E}-03$ & $1.4 \mathrm{E}-02$ & $3.7 \mathrm{E}+00$ \\
575 & $3.1 \mathrm{E}-02$ & $3.1 \mathrm{E}-03$ & $7.9 \mathrm{E}-03$ & $1.6 \mathrm{E}-02$ & $4.2 \mathrm{E}+00$ \\
750 & $3.1 \mathrm{E}-02$ & $3.1 \mathrm{E}-03$ & $8.0 \mathrm{E}-03$ & $1.6 \mathrm{E}-02$ & $4.3 \mathrm{E}+00$ \\
1000 & $3.1 \mathrm{E}-02$ & $3.1 \mathrm{E}-03$ & $8.0 \mathrm{E}-03$ & $1.6 \mathrm{E}-02$ & $4.3 \mathrm{E}+00$ \\
1025 & $7.3 \mathrm{E}-03$ & $5.8 \mathrm{E}-04$ & $4.2 \mathrm{E}-03$ & $3.3 \mathrm{E}-03$ & $1.1 \mathrm{E}+00$ \\
1050 & $6.1 \mathrm{E}-03$ & $4.9 \mathrm{E}-04$ & $3.5 \mathrm{E}-03$ & $2.8 \mathrm{E}-03$ & $8.8 \mathrm{E}-01$ \\
1100 & $4.3 \mathrm{E}-03$ & $3.5 \mathrm{E}-04$ & $2.5 \mathrm{E}-03$ & $2.0 \mathrm{E}-03$ & $6.2 \mathrm{E}-01$ \\
1200 & $2.2 \mathrm{E}-03$ & $1.7 \mathrm{E}-04$ & $1.2 \mathrm{E}-03$ & $9.9 \mathrm{E}-04$ & $3.1 \mathrm{E}-01$ \\
1400 & $5.4 \mathrm{E}-04$ & $4.3 \mathrm{E}-05$ & $3.1 \mathrm{E}-04$ & $2.5 \mathrm{E}-04$ & $7.8 \mathrm{E}-02$ \\
1600 & $1.4 \mathrm{E}-04$ & $1.1 \mathrm{E}-05$ & $7.8 \mathrm{E}-05$ & $6.2 \mathrm{E}-05$ & $2.0 \mathrm{E}-02$ \\
2000 & $8.5 \mathrm{E}-06$ & $6.7 \mathrm{E}-07$ & $4.9 \mathrm{E}-06$ & $3.9 \mathrm{E}-06$ & $1.2 \mathrm{E}-03$ \\
2500 & $2.6 \mathrm{E}-07$ & $2.1 \mathrm{E}-08$ & $1.5 \mathrm{E}-07$ & $1.2 \mathrm{E}-07$ & $3.8 \mathrm{E}-05$ \\
3000 & $8.3 \mathrm{E}-09$ & $6.5 \mathrm{E}-10$ & $4.7 \mathrm{E}-09$ & $3.8 \mathrm{E}-09$ & $1.2 \mathrm{E}-06$ \\
& & & & & \\
\hline
\end{tabular}

AABDU Dose from Beef Ingestion in Zone A Due to Atmospheric Release (Sv $\mathrm{yr}^{-1}$ )

\begin{tabular}{rrrrrrr}
\hline Time (Yr) & U-238\&4 & Th-230 & Ra-226 & Pb-210 & Po-210 & Chain \\
\hline 1 & $4.6 \mathrm{E}-08$ & $4.2 \mathrm{E}-08$ & $3.4 \mathrm{E}-08$ & $7.9 \mathrm{E}-07$ & $9.4 \mathrm{E}-05$ & $9.5 \mathrm{E}-05$ \\
20 & $4.8 \mathrm{E}-08$ & $4.4 \mathrm{E}-08$ & $4.0 \mathrm{E}-08$ & $8.2 \mathrm{E}-07$ & $9.9 \mathrm{E}-05$ & $1.0 \mathrm{E}-04$ \\
25 & $4.8 \mathrm{E}-08$ & $4.4 \mathrm{E}-08$ & $4.2 \mathrm{E}-08$ & $8.3 \mathrm{E}-07$ & $1.0 \mathrm{E}-04$ & $1.0 \mathrm{E}-04$ \\
30 & $4.9 \mathrm{E}-08$ & $4.5 \mathrm{E}-08$ & $4.3 \mathrm{E}-08$ & $8.3 \mathrm{E}-07$ & $1.0 \mathrm{E}-04$ & $1.0 \mathrm{E}-04$ \\
62 & $5.2 \mathrm{E}-08$ & $4.7 \mathrm{E}-08$ & $5.2 \mathrm{E}-08$ & $8.8 \mathrm{E}-07$ & $1.1 \mathrm{E}-04$ & $1.1 \mathrm{E}-04$ \\
125 & $5.6 \mathrm{E}-08$ & $4.9 \mathrm{E}-08$ & $6.5 \mathrm{E}-08$ & $9.4 \mathrm{E}-07$ & $1.2 \mathrm{E}-04$ & $1.2 \mathrm{E}-04$ \\
575 & $6.3 \mathrm{E}-08$ & $5.4 \mathrm{E}-08$ & $8.7 \mathrm{E}-08$ & $1.0 \mathrm{E}-06$ & $1.3 \mathrm{E}-04$ & $1.3 \mathrm{E}-04$ \\
750 & $6.3 \mathrm{E}-08$ & $5.4 \mathrm{E}-08$ & $8.8 \mathrm{E}-08$ & $1.0 \mathrm{E}-06$ & $1.3 \mathrm{E}-04$ & $1.3 \mathrm{E}-04$ \\
1000 & $6.3 \mathrm{E}-08$ & $5.4 \mathrm{E}-08$ & $\mathbf{8 . 8 E}-08$ & $1.0 \mathrm{E}-06$ & $1.3 \mathrm{E}-04$ & $1.3 \mathrm{E}-04$ \\
1025 & $1.5 \mathrm{E}-08$ & $1.0 \mathrm{E}-08$ & $4.6 \mathrm{E}-08$ & $2.2 \mathrm{E}-07$ & $3.2 \mathrm{E}-05$ & $3.3 \mathrm{E}-05$ \\
1050 & $1.2 \mathrm{E}-08$ & $8.5 \mathrm{E}-09$ & $3.9 \mathrm{E}-08$ & $1.8 \mathrm{E}-07$ & $2.7 \mathrm{E}-05$ & $2.8 \mathrm{E}-05$ \\
1100 & $8.8 \mathrm{E}-09$ & $6.0 \mathrm{E}-09$ & $2.7 \mathrm{E}-08$ & $1.3 \mathrm{E}-07$ & $1.9 \mathrm{E}-05$ & $1.9 \mathrm{E}-05$ \\
1200 & $4.4 \mathrm{E}-09$ & $3.0 \mathrm{E}-09$ & $1.4 \mathrm{E}-08$ & $6.5 \mathrm{E}-08$ & $9.6 \mathrm{E}-06$ & $9.7 \mathrm{E}-06$ \\
1400 & $1.1 \mathrm{E}-09$ & $7.5 \mathrm{E}-10$ & $3.4 \mathrm{E}-09$ & $1.6 \mathrm{E}-08$ & $2.4 \mathrm{E}-06$ & $2.4 \mathrm{E}-06$ \\
1600 & $2.7 \mathrm{E}-10$ & $1.9 \mathrm{E}-10$ & $8.6 \mathrm{E}-10$ & $4.0 \mathrm{E}-09$ & $6.0 \mathrm{E}-07$ & $6.1 \mathrm{E}-07$ \\
2000 & $1.7 \mathrm{E}-11$ & $1.2 \mathrm{E}-11$ & $5.3 \mathrm{E}-11$ & $2.5 \mathrm{E}-10$ & $3.8 \mathrm{E}-08$ & $3.8 \mathrm{E}-08$ \\
2500 & $5.3 \mathrm{E}-13$ & $3.6 \mathrm{E}-13$ & $1.7 \mathrm{E}-12$ & $7.9 \mathrm{E}-12$ & $1.2 \mathrm{E}-09$ & $1.2 \mathrm{E}-09$ \\
3000 & $1.7 \mathrm{E}-14$ & $1.1 \mathrm{E}-14$ & $5.2 \mathrm{E}-14$ & $2.5 \mathrm{E}-13$ & $3.7 \mathrm{E}-11$ & $3.7 \mathrm{E}-11$ \\
\hline
\end{tabular}


AABRU Cancer Risk from Beef Ingestion in Zone A Due to Atmospheric Release

\begin{tabular}{rllllll}
\hline Time (yr) & U-238\&4 & Th-230 & Ra-226 & Pb-210 & Po-210 & Chain \\
\hline 1 & $4.7 \mathrm{E}-08$ & $3.0 \mathrm{E}-09$ & $3.4 \mathrm{E}-08$ & $4.3 \mathrm{E}-05$ & $7.2 \mathrm{E}-07$ & $4.4 \mathrm{E}-05$ \\
20 & $4.9 \mathrm{E}-08$ & $3.1 \mathrm{E}-09$ & $4.1 \mathrm{E}-08$ & $4.3 \mathrm{E}-05$ & $2.9 \mathrm{E}-06$ & $4.6 \mathrm{E}-05$ \\
25 & $5.0 \mathrm{E}-08$ & $3.1 \mathrm{E}-09$ & $4.3 \mathrm{E}-08$ & $4.3 \mathrm{E}-05$ & $3.4 \mathrm{E}-06$ & $4.7 \mathrm{E}-05$ \\
30 & $5.0 \mathrm{E}-08$ & $3.1 \mathrm{E}-09$ & $4.4 \mathrm{E}-08$ & $4.3 \mathrm{E}-05$ & $4.0 \mathrm{E}-06$ & $4.7 \mathrm{E}-05$ \\
62 & $5.3 \mathrm{E}-08$ & $3.3 \mathrm{E}-09$ & $5.3 \mathrm{E}-08$ & $4.3 \mathrm{E}-05$ & $6.8 \mathrm{E}-06$ & $5.0 \mathrm{E}-05$ \\
125 & $5.7 \mathrm{E}-08$ & $3.4 \mathrm{E}-09$ & $6.6 \mathrm{E}-08$ & $4.3 \mathrm{E}-05$ & $1.1 \mathrm{E}-05$ & $5.5 \mathrm{E}-05$ \\
575 & $6.4 \mathrm{E}-08$ & $3.8 \mathrm{E}-09$ & $8.9 \mathrm{E}-08$ & $4.4 \mathrm{E}-05$ & $1.8 \mathrm{E}-05$ & $6.2 \mathrm{E}-05$ \\
750 & $6.5 \mathrm{E}-08$ & $3.8 \mathrm{E}-09$ & $8.9 \mathrm{E}-08$ & $4.3 \mathrm{E}-05$ & $1.8 \mathrm{E}-05$ & $6.2 \mathrm{E}-05$ \\
1000 & $6.5 \mathrm{E}-08$ & $3.8 \mathrm{E}-09$ & $9.0 \mathrm{E}-08$ & $4.3 \mathrm{E}-05$ & $1.8 \mathrm{E}-05$ & $6.2 \mathrm{E}-05$ \\
1025 & $1.5 \mathrm{E}-08$ & $7.1 \mathrm{E}-10$ & $4.7 \mathrm{E}-08$ & $1.6 \mathrm{E}-07$ & $1.5 \mathrm{E}-05$ & $1.5 \mathrm{E}-05$ \\
1050 & $1.3 \mathrm{E}-08$ & $6.0 \mathrm{E}-10$ & $3.9 \mathrm{E}-08$ & $1.4 \mathrm{E}-07$ & $1.3 \mathrm{E}-05$ & $1.3 \mathrm{E}-05$ \\
1100 & $9.0 \mathrm{E}-09$ & $4.2 \mathrm{E}-10$ & $2.8 \mathrm{E}-08$ & $9.8 \mathrm{E}-08$ & $8.9 \mathrm{E}-06$ & $9.1 \mathrm{E}-06$ \\
1200 & $4.5 \mathrm{E}-09$ & $2.1 \mathrm{E}-10$ & $1.4 \mathrm{E}-08$ & $4.9 \mathrm{E}-08$ & $4.5 \mathrm{E}-06$ & $4.5 \mathrm{E}-06$ \\
1400 & $1.1 \mathrm{E}-09$ & $5.3 \mathrm{E}-11$ & $3.5 \mathrm{E}-09$ & $1.2 \mathrm{E}-08$ & $1.1 \mathrm{E}-06$ & $1.1 \mathrm{E}-06$ \\
1600 & $2.8 \mathrm{E}-10$ & $1.3 \mathrm{E}-11$ & $8.7 \mathrm{E}-10$ & $3.0 \mathrm{E}-09$ & $2.8 \mathrm{E}-07$ & $2.8 \mathrm{E}-07$ \\
2000 & $1.8 \mathrm{E}-11$ & $8.2 \mathrm{E}-13$ & $5.4 \mathrm{E}-11$ & $1.9 \mathrm{E}-10$ & $1.7 \mathrm{E}-08$ & $1.8 \mathrm{E}-08$ \\
2500 & $5.5 \mathrm{E}-13$ & $2.5 \mathrm{E}-14$ & $1.7 \mathrm{E}-12$ & $5.9 \mathrm{E}-12$ & $5.4 \mathrm{E}-10$ & $5.5 \mathrm{E}-10$ \\
3000 & $1.7 \mathrm{E}-14$ & $7.9 \mathrm{E}-16$ & $5.3 \mathrm{E}-14$ & $1.9 \mathrm{E}-13$ & $1.7 \mathrm{E}-11$ & $1.7 \mathrm{E}-11$ \\
\hline
\end{tabular}

AABCM Concentration in Beef

( $\mathrm{mg} \mathrm{kg}^{-1}$ ) in Zone $\mathrm{A}$ Due to Atmospheric Release

\begin{tabular}{rrrr}
\hline Time $(y r)$ & As & Cr & $N i$ \\
\hline 1 & $2.2 E-02$ & $4.3 E-05$ & $2.2 E-06$ \\
20 & $2.3 E-02$ & $4.6 E-05$ & $2.3 E-06$ \\
25 & $2.3 E-02$ & $4.7 E-05$ & $2.3 E-06$ \\
30 & $2.3 E-02$ & $4.8 E-05$ & $2.3 E-06$ \\
62 & $2.5 E-02$ & $5.1 E-05$ & $2.5 E-06$ \\
125 & $2.6 E-02$ & $5.7 E-05$ & $2.7 E-06$ \\
575 & $3.0 E-02$ & $6.6 E-05$ & $3.0 E-06$ \\
750 & $3.0 E-02$ & $6.7 E-05$ & $3.0 E-06$ \\
1000 & $3.0 E-02$ & $6.7 E-05$ & $3.0 E-06$ \\
1025 & $7.0 E-03$ & $2.0 E-05$ & $7.5 E-07$ \\
1050 & $5.9 E-03$ & $1.7 E-05$ & $6.3 E-07$ \\
1100 & $4.2 E-03$ & $1.2 E-05$ & $4.5 E-07$ \\
1200 & $2.1 E-03$ & $6.0 E-06$ & $2.2 E-07$ \\
1400 & $5.2 E-04$ & $1.5 E-06$ & $5.6 E-08$ \\
1600 & $1.3 E-04$ & $3.7 E-07$ & $1.4 E-08$ \\
2000 & $8.1 E-06$ & $2.3 E-08$ & $8.7 E-10$ \\
2500 & $2.5 E-07$ & $7.3 E-10$ & $2.7 E-11$ \\
3000 & $7.9 E-09$ & $2.3 E-11$ & $8.5 E-13$ \\
& & & \\
\hline
\end{tabular}

AABIM Intake from Beef Ingestion in Zone A Due to Atmospheric Release ( $\mathrm{mg} \mathrm{yr}^{-1}$ )

\begin{tabular}{rrrr}
\hline Time $(y x)$ & As & Cr & $N i$ \\
\hline 1 & $1.1 \mathrm{E}+00$ & $2.2 \mathrm{E}-03$ & $1.1 \mathrm{E}-04$ \\
20 & $1.1 \mathrm{E}+00$ & $2.3 \mathrm{E}-03$ & $1.1 \mathrm{E}-04$ \\
25 & $1.1 \mathrm{E}+00$ & $2.3 \mathrm{E}-03$ & $1.1 \mathrm{E}-04$ \\
30 & $1.2 \mathrm{E}+00$ & $2.4 \mathrm{E}-03$ & $1.2 \mathrm{E}-04$ \\
62 & $1.2 \mathrm{E}+00$ & $2.6 \mathrm{E}-03$ & $1.2 \mathrm{E}-04$ \\
125 & $1.3 \mathrm{E}+00$ & $2.8 \mathrm{E}-03$ & $1.3 \mathrm{E}-04$ \\
575 & $1.5 \mathrm{E}+00$ & $3.3 \mathrm{E}-03$ & $1.5 \mathrm{E}-04$ \\
750 & $1.5 \mathrm{E}+00$ & $3.3 \mathrm{E}-03$ & $1.5 \mathrm{E}-04$ \\
1000 & $1.5 \mathrm{E}+00$ & $3.3 \mathrm{E}-03$ & $1.5 \mathrm{E}-04$ \\
1025 & $3.5 \mathrm{E}-01$ & $1.0 \mathrm{E}-03$ & $3.8 \mathrm{E}-05$ \\
1050 & $2.9 \mathrm{E}-01$ & $8.4 \mathrm{E}-04$ & $3.2 \mathrm{E}-05$ \\
1100 & $2.1 \mathrm{E}-01$ & $6.0 \mathrm{E}-04$ & $2.2 \mathrm{E}-05$ \\
1200 & $1.0 \mathrm{E}-01$ & $3.0 \mathrm{E}-04$ & $1.1 \mathrm{E}-05$ \\
1400 & $2.6 \mathrm{E}-02$ & $7.4 \mathrm{E}-05$ & $2.8 \mathrm{E}-06$ \\
1600 & $6.5 \mathrm{E}-03$ & $1.9 \mathrm{E}-05$ & $7.0 \mathrm{E}-07$ \\
2000 & $4.1 \mathrm{E}-04$ & $1.2 \mathrm{E}-06$ & $4.4 \mathrm{E}-08$ \\
2500 & $1.3 \mathrm{E}-05$ & $3.6 \mathrm{E}-08$ & $1.4 \mathrm{E}-09$ \\
3000 & $4.0 \mathrm{E}-07$ & $1.1 \mathrm{E}-09$ & $4.3 \mathrm{E}-11$ \\
& & &
\end{tabular}


AABRM Cancer Risk from Beef

Ingestion in Zone $A$ Due to

Atmospheric Release

\begin{tabular}{rrrr}
\hline Time $(y r)$ & As & $\mathrm{Cr}$ & $\mathrm{Ni}$ \\
\hline 1 & $1.9 \mathrm{E}-02$ & $0.0 \mathrm{E}+00$ & $0.0 \mathrm{E}+00$ \\
20 & $2.0 \mathrm{E}-02$ & $0.0 \mathrm{E}+00$ & $0.0 \mathrm{E}+00$ \\
25 & $2.0 \mathrm{E}-02$ & $0.0 \mathrm{E}+00$ & $0.0 \mathrm{E}+00$ \\
30 & $2.0 \mathrm{E}-02$ & $0.0 \mathrm{E}+00$ & $0.0 \mathrm{E}+00$ \\
62 & $2.1 \mathrm{E}-02$ & $0.0 \mathrm{E}+00$ & $0.0 \mathrm{E}+00$ \\
125 & $2.3 \mathrm{E}-02$ & $0.0 \mathrm{E}+00$ & $0.0 \mathrm{E}+00$ \\
575 & $2.6 \mathrm{E}-02$ & $0.0 \mathrm{E}+00$ & $0.0 \mathrm{E}+00$ \\
750 & $2.6 \mathrm{E}-02$ & $0.0 \mathrm{E}+00$ & $0.0 \mathrm{E}+00$ \\
1000 & $2.6 \mathrm{E}-02$ & $0.0 \mathrm{E}+00$ & $0.0 \mathrm{E}+00$ \\
1025 & $6.1 \mathrm{E}-03$ & $0.0 \mathrm{E}+00$ & $0.0 \mathrm{E}+00$ \\
1050 & $5.2 \mathrm{E}-03$ & $0.0 \mathrm{E}+00$ & $0.0 \mathrm{E}+00$ \\
1100 & $3.6 \mathrm{E}-03$ & $0.0 \mathrm{E}+00$ & $0.0 \mathrm{E}+00$ \\
1200 & $1.8 \mathrm{E}-03$ & $0.0 \mathrm{E}+00$ & $0.0 \mathrm{E}+00$ \\
1400 & $4.6 \mathrm{E}-04$ & $0.0 \mathrm{E}+00$ & $0.0 \mathrm{E}+00$ \\
1600 & $1.1 \mathrm{E}-04$ & $0.0 \mathrm{E}+00$ & $0.0 \mathrm{E}+00$ \\
2000 & $7.1 \mathrm{E}-06$ & $0.0 \mathrm{E}+00$ & $0.0 \mathrm{E}+00$ \\
2500 & $2.2 \mathrm{E}-07$ & $0.0 \mathrm{E}+00$ & $0.0 \mathrm{E}+00$ \\
3000 & $7.0 \mathrm{E}-09$ & $0.0 \mathrm{E}+00$ & $0.0 \mathrm{E}+00$ \\
& & & \\
\hline
\end{tabular}

ABBCU Concentration in Beef ( $\mathrm{Bq} \mathrm{kg}^{-1}$ ) in Zone $B$ Due to Atmospheric Release

\begin{tabular}{rrrrrr}
\hline Time $(y r)$ & $\mathrm{U}-238 \& 4$ & $\mathrm{Th}-230$ & $\mathrm{Ra}-226$ & $\mathrm{~Pb}-210$ & Po-210 \\
\hline 1 & $4.1 \mathrm{E}-03$ & $4.4 \mathrm{E}-04$ & $5.5 \mathrm{E}-04$ & $2.2 \mathrm{E}-03$ & $5.5 \mathrm{E}-01$ \\
20 & $4.3 \mathrm{E}-03$ & $4.5 \mathrm{E}-04$ & $6.6 \mathrm{E}-04$ & $2.3 \mathrm{E}-03$ & $5.8 \mathrm{E}-01$ \\
25 & $4.3 \mathrm{E}-03$ & $4.6 \mathrm{E}-04$ & $6.9 \mathrm{E}-04$ & $2.3 \mathrm{E}-03$ & $5.8 \mathrm{E}-01$ \\
30 & $4.4 \mathrm{E}-03$ & $4.6 \mathrm{E}-04$ & $7.2 \mathrm{E}-04$ & $2.3 \mathrm{E}-03$ & $5.9 \mathrm{E}-01$ \\
62 & $4.6 \mathrm{E}-03$ & $4.8 \mathrm{E}-04$ & $8.6 \mathrm{E}-04$ & $2.4 \mathrm{E}-03$ & $6.3 \mathrm{E}-01$ \\
125 & $5.0 \mathrm{E}-03$ & $5.1 \mathrm{E}-04$ & $1.1 \mathrm{E}-03$ & $2.6 \mathrm{E}-03$ & $6.8 \mathrm{E}-01$ \\
575 & $5.6 \mathrm{E}-03$ & $5.6 \mathrm{E}-04$ & $1.4 \mathrm{E}-03$ & $2.9 \mathrm{E}-03$ & $7.7 \mathrm{E}-01$ \\
750 & $5.6 \mathrm{E}-03$ & $5.6 \mathrm{E}-04$ & $1.4 \mathrm{E}-03$ & $2.9 \mathrm{E}-03$ & $7.7 \mathrm{E}-01$ \\
1000 & $5.6 \mathrm{E}-03$ & $5.6 \mathrm{E}-04$ & $1.5 \mathrm{E}-03$ & $2.9 \mathrm{E}-03$ & $7.7 \mathrm{E}-01$ \\
1025 & $1.3 \mathrm{E}-03$ & $1.1 \mathrm{E}-04$ & $7.6 \mathrm{E}-04$ & $6.1 \mathrm{E}-04$ & $1.9 \mathrm{E}-01$ \\
1050 & $1.1 \mathrm{E}-03$ & $8.8 \mathrm{E}-05$ & $6.4 \mathrm{E}-04$ & $5.1 \mathrm{E}-04$ & $1.6 \mathrm{E}-01$ \\
1100 & $7.8 \mathrm{E}-04$ & $6.2 \mathrm{E}-05$ & $4.5 \mathrm{E}-04$ & $3.6 \mathrm{E}-04$ & $1.1 \mathrm{E}-01$ \\
1200 & $3.9 \mathrm{E}-04$ & $3.1 \mathrm{E}-05$ & $2.3 \mathrm{E}-04$ & $1.8 \mathrm{E}-04$ & $5.6 \mathrm{E}-02$ \\
1400 & $9.8 \mathrm{E}-05$ & $7.8 \mathrm{E}-06$ & $5.7 \mathrm{E}-05$ & $4.5 \mathrm{E}-05$ & $1.4 \mathrm{E}-02$ \\
1600 & $2.5 \mathrm{E}-05$ & $1.9 \mathrm{E}-06$ & $1.4 \mathrm{E}-05$ & $1.1 \mathrm{E}-05$ & $3.5 \mathrm{E}-03$ \\
2000 & $1.5 \mathrm{E}-06$ & $1.2 \mathrm{E}-07$ & $8.8 \mathrm{E}-07$ & $7.1 \mathrm{E}-07$ & $2.2 \mathrm{E}-04$ \\
2500 & $4.8 \mathrm{E}-08$ & $3.8 \mathrm{E}-09$ & $2.8 \mathrm{E}-08$ & $2.2 \mathrm{E}-08$ & $6.9 \mathrm{E}-06$ \\
3000 & $1.5 \mathrm{E}-09$ & $1.2 \mathrm{E}-10$ & $8.6 \mathrm{E}-10$ & $6.9 \mathrm{E}-10$ & $2.1 \mathrm{E}-07$ \\
& & & & & \\
\hline
\end{tabular}


ABBDU Dose from Beef Ingestion in Zone $B$ Due to Atmospheric Release (Sv $\mathrm{Yr}^{-1}$ )

\begin{tabular}{rcccccc}
\hline Time (yr) & $\mathrm{U}-238 \mathrm{E} 4$ & Th-230 & Ra-226 & Pb-210 & Po-210 & Chain \\
\hline 1 & $8.3 \mathrm{E}-09$ & $7.7 \mathrm{E}-09$ & $6.1 \mathrm{E}-09$ & $1.4 \mathrm{E}-07$ & $1.7 \mathrm{E}-05$ & $1.7 \mathrm{E}-05$ \\
20 & $8.7 \mathrm{E}-09$ & $7.9 \mathrm{E}-09$ & $7.3 \mathrm{E}-09$ & $1.5 \mathrm{E}-07$ & $1.8 \mathrm{E}-05$ & $1.8 \mathrm{E}-05$ \\
25 & $8.8 \mathrm{E}-09$ & $8.0 \mathrm{E}-09$ & $7.6 \mathrm{E}-09$ & $1.5 \mathrm{E}-07$ & $1.8 \mathrm{E}-05$ & $1.8 \mathrm{E}-05$ \\
30 & $8.8 \mathrm{E}-09$ & $8.1 \mathrm{E}-09$ & $7.9 \mathrm{E}-09$ & $1.5 \mathrm{E}-07$ & $1.8 \mathrm{E}-05$ & $1.8 \mathrm{E}-05$ \\
62 & $9.4 \mathrm{E}-09$ & $8.4 \mathrm{E}-09$ & $9.5 \mathrm{E}-09$ & $1.6 \mathrm{E}-07$ & $1.9 \mathrm{E}-05$ & $2.0 \mathrm{E}-05$ \\
125 & $1.0 \mathrm{E}-08$ & $8.9 \mathrm{E}-09$ & $1.2 \mathrm{E}-08$ & $1.7 \mathrm{E}-07$ & $2.1 \mathrm{E}-05$ & $2.1 \mathrm{E}-05$ \\
575 & $1.1 \mathrm{E}-08$ & $9.8 \mathrm{E}-09$ & $1.6 \mathrm{E}-08$ & $1.9 \mathrm{E}-07$ & $2.4 \mathrm{E}-05$ & $2.4 \mathrm{E}-05$ \\
750 & $1.1 \mathrm{E}-08$ & $9.8 \mathrm{E}-09$ & $1.6 \mathrm{E}-08$ & $1.9 \mathrm{E}-07$ & $2.4 \mathrm{E}-05$ & $2.4 \mathrm{E}-05$ \\
1000 & $1.1 \mathrm{E}-08$ & $9.8 \mathrm{E}-09$ & $1.6 \mathrm{E}-08$ & $1.9 \mathrm{E}-07$ & $2.4 \mathrm{E}-05$ & $2.4 \mathrm{E}-05$ \\
1025 & $2.7 \mathrm{E}-09$ & $1.8 \mathrm{E}-09$ & $8.4 \mathrm{E}-09$ & $4.0 \mathrm{E}-08$ & $5.9 \mathrm{E}-06$ & $5.9 \mathrm{E}-06$ \\
1050 & $2.2 \mathrm{E}-09$ & $1.5 \mathrm{E}-09$ & $7.0 \mathrm{E}-09$ & $3.3 \mathrm{E}-08$ & $5.0 \mathrm{E}-06$ & $5.0 \mathrm{E}-06$ \\
1100 & $1.6 \mathrm{E}-09$ & $1.1 \mathrm{E}-09$ & $5.0 \mathrm{E}-09$ & $2.4 \mathrm{E}-08$ & $3.5 \mathrm{E}-06$ & $3.5 \mathrm{E}-06$ \\
1200 & $8.0 \mathrm{E}-10$ & $5.5 \mathrm{E}-10$ & $2.5 \mathrm{E}-09$ & $1.2 \mathrm{E}-08$ & $1.8 \mathrm{E}-06$ & $1.8 \mathrm{E}-06$ \\
1400 & $2.0 \mathrm{E}-10$ & $1.4 \mathrm{E}-10$ & $6.2 \mathrm{E}-10$ & $2.9 \mathrm{E}-09$ & $4.4 \mathrm{E}-07$ & $4.4 \mathrm{E}-07$ \\
1600 & $5.0 \mathrm{E}-11$ & $3.4 \mathrm{E}-11$ & $1.6 \mathrm{E}-10$ & $7.3 \mathrm{E}-10$ & $1.1 \mathrm{E}-07$ & $1.1 \mathrm{E}-07$ \\
2000 & $3.1 \mathrm{E}-12$ & $2.1 \mathrm{E}-12$ & $9.7 \mathrm{E}-12$ & $4.6 \mathrm{E}-11$ & $6.8 \mathrm{E}-09$ & $6.9 \mathrm{E}-09$ \\
2500 & $9.7 \mathrm{E}-14$ & $6.6 \mathrm{E}-14$ & $3.0 \mathrm{E}-13$ & $1.4 \mathrm{E}-12$ & $2.1 \mathrm{E}-10$ & $2.2 \mathrm{E}-10$ \\
3000 & $3.0 \mathrm{E}-15$ & $2.1 \mathrm{E}-15$ & $9.4 \mathrm{E}-15$ & $4.5 \mathrm{E}-14$ & $6.6 \mathrm{E}-12$ & $6.7 \mathrm{E}-12$ \\
\hline
\end{tabular}

ABBRU Cancer Risk from Beef Ingestion in zone $B$ Due to Atmospheric Release

\begin{tabular}{rrrrrrr}
\hline Time (Yr) & U-238\&4 & Th-230 & Ra-226 & Pb-210 & Po-210 & Chain \\
\hline 1 & $8.5 \mathrm{E}-09$ & $5.4 \mathrm{E}-10$ & $6.2 \mathrm{E}-09$ & $7.9 \mathrm{E}-06$ & $1.3 \mathrm{E}-07$ & $8.0 \mathrm{E}-06$ \\
20 & $8.9 \mathrm{E}-09$ & $5.6 \mathrm{E}-10$ & $7.4 \mathrm{E}-09$ & $7.9 \mathrm{E}-06$ & $5.3 \mathrm{E}-07$ & $8.4 \mathrm{E}-06$ \\
25 & $9.0 \mathrm{E}-09$ & $5.6 \mathrm{E}-10$ & $7.7 \mathrm{E}-09$ & $7.9 \mathrm{E}-06$ & $6.2 \mathrm{E}-07$ & $8.5 \mathrm{E}-06$ \\
30 & $9.1 \mathrm{E}-09$ & $5.7 \mathrm{E}-10$ & $8.0 \mathrm{E}-09$ & $7.9 \mathrm{E}-06$ & $7.2 \mathrm{E}-07$ & $8.6 \mathrm{E}-06$ \\
62 & $9.6 \mathrm{E}-09$ & $5.9 \mathrm{E}-10$ & $9.7 \mathrm{E}-09$ & $7.9 \mathrm{E}-06$ & $1.2 \mathrm{E}-06$ & $9.1 \mathrm{E}-06$ \\
125 & $1.0 \mathrm{E}-08$ & $6.2 \mathrm{E}-10$ & $1.2 \mathrm{E}-08$ & $7.9 \mathrm{E}-06$ & $2.0 \mathrm{E}-06$ & $9.9 \mathrm{E}-06$ \\
575 & $1.2 \mathrm{E}-08$ & $6.8 \mathrm{E}-10$ & $1.6 \mathrm{E}-08$ & $7.9 \mathrm{E}-06$ & $3.3 \mathrm{E}-06$ & $1.1 \mathrm{E}-05$ \\
750 & $1.2 \mathrm{E}-08$ & $6.9 \mathrm{E}-10$ & $1.6 \mathrm{E}-08$ & $7.9 \mathrm{E}-06$ & $3.3 \mathrm{E}-06$ & $1.1 \mathrm{E}-05$ \\
1000 & $1.2 \mathrm{E}-08$ & $6.9 \mathrm{E}-10$ & $1.6 \mathrm{E}-08$ & $7.9 \mathrm{E}-06$ & $3.4 \mathrm{E}-06$ & $1.1 \mathrm{E}-05$ \\
1025 & $2.8 \mathrm{E}-09$ & $1.3 \mathrm{E}-10$ & $8.5 \mathrm{E}-09$ & $3.0 \mathrm{E}-08$ & $2.7 \mathrm{E}-06$ & $2.8 \mathrm{E}-06$ \\
1050 & $2.3 \mathrm{E}-09$ & $1.1 \mathrm{E}-10$ & $7.2 \mathrm{E}-09$ & $2.5 \mathrm{E}-08$ & $2.3 \mathrm{E}-06$ & $2.3 \mathrm{E}-06$ \\
1100 & $1.6 \mathrm{E}-09$ & $7.7 \mathrm{E}-11$ & $5.1 \mathrm{E}-09$ & $1.8 \mathrm{E}-08$ & $1.6 \mathrm{E}-06$ & $1.6 \mathrm{E}-06$ \\
1200 & $8.2 \mathrm{E}-10$ & $3.8 \mathrm{E}-11$ & $2.5 \mathrm{E}-09$ & $8.9 \mathrm{E}-09$ & $8.1 \mathrm{E}-07$ & $8.2 \mathrm{E}-07$ \\
1400 & $2.0 \mathrm{E}-10$ & $9.6 \mathrm{E}-12$ & $6.3 \mathrm{E}-10$ & $2.2 \mathrm{E}-09$ & $2.0 \mathrm{E}-07$ & $2.1 \mathrm{E}-07$ \\
1600 & $5.1 \mathrm{E}-11$ & $2.4 \mathrm{E}-12$ & $1.6 \mathrm{E}-10$ & $5.5 \mathrm{E}-10$ & $5.1 \mathrm{E}-08$ & $5.1 \mathrm{E}-08$ \\
2000 & $3.2 \mathrm{E}-12$ & $1.5 \mathrm{E}-13$ & $9.9 \mathrm{E}-12$ & $3.5 \mathrm{E}-11$ & $3.2 \mathrm{E}-09$ & $3.2 \mathrm{E}-09$ \\
2500 & $1.0 \mathrm{E}-13$ & $4.6 \mathrm{E}-15$ & $3.1 \mathrm{E}-13$ & $1.1 \mathrm{E}-12$ & $9.9 \mathrm{E}-11$ & $1.0 \mathrm{E}-10$ \\
3000 & $3.1 \mathrm{E}-15$ & $1.4 \mathrm{E}-16$ & $9.6 \mathrm{E}-15$ & $3.4 \mathrm{E}-14$ & $3.1 \mathrm{E}-12$ & $3.1 \mathrm{E}-12$ \\
\hline
\end{tabular}


ABBCM Concentration in Beef ( $\mathrm{mg} \mathrm{kg}^{-1}$ ) in Zone $\mathrm{B}$ Due to Atmospheric Release

\begin{tabular}{rrrr}
\hline Time $(y r)$ & As & Cr & Ni \\
\hline 1 & $3.9 \mathrm{E}-03$ & $7.8 \mathrm{E}-06$ & $3.9 \mathrm{E}-07$ \\
20 & $4.1 \mathrm{E}-03$ & $8.4 \mathrm{E}-06$ & $4.1 \mathrm{E}-07$ \\
25 & $4.1 \mathrm{E}-03$ & $8.5 \mathrm{E}-06$ & $4.2 \mathrm{E}-07$ \\
30 & $4.2 \mathrm{E}-03$ & $8.6 \mathrm{E}-06$ & $4.2 \mathrm{E}-07$ \\
62 & $4.4 \mathrm{E}-03$ & $9.3 \mathrm{E}-06$ & $4.5 \mathrm{E}-07$ \\
125 & $4.8 \mathrm{E}-03$ & $1.0 \mathrm{E}-05$ & $4.8 \mathrm{E}-07$ \\
575 & $5.4 \mathrm{E}-03$ & $1.2 \mathrm{E}-05$ & $5.5 \mathrm{E}-07$ \\
750 & $5.4 \mathrm{E}-03$ & $1.2 \mathrm{E}-05$ & $5.5 \mathrm{E}-07$ \\
1000 & $5.4 \mathrm{E}-03$ & $1.2 \mathrm{E}-05$ & $5.5 \mathrm{E}-07$ \\
1025 & $1.3 \mathrm{E}-03$ & $3.6 \mathrm{E}-06$ & $1.4 \mathrm{E}-07$ \\
1050 & $1.1 \mathrm{E}-03$ & $3.0 \mathrm{E}-06$ & $1.1 \mathrm{E}-07$ \\
1100 & $7.5 \mathrm{E}-04$ & $2.2 \mathrm{E}-06$ & $8.1 \mathrm{E}-08$ \\
1200 & $3.8 \mathrm{E}-04$ & $1.1 \mathrm{E}-06$ & $4.0 \mathrm{E}-08$ \\
1400 & $9.4 \mathrm{E}-05$ & $2.7 \mathrm{E}-07$ & $1.0 \mathrm{E}-08$ \\
1600 & $2.3 \mathrm{E}-05$ & $6.7 \mathrm{E}-08$ & $2.5 \mathrm{E}-09$ \\
2000 & $1.5 \mathrm{E}-06$ & $4.2 \mathrm{E}-09$ & $1.6 \mathrm{E}-10$ \\
2500 & $4.6 \mathrm{E}-08$ & $1.3 \mathrm{E}-10$ & $4.9 \mathrm{E}-12$ \\
3000 & $1.4 \mathrm{E}-09$ & $4.1 \mathrm{E}-12$ & $1.5 \mathrm{E}-13$ \\
& & & \\
\hline
\end{tabular}

ABBIM Intake from Beef Ingestion in Zone $B$ Due to

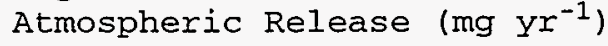

\begin{tabular}{rrrr}
\hline Time $(Y r)$ & As & Cr & Ni \\
\hline 1 & $2.0 \mathrm{E}-01$ & $3.9 \mathrm{E}-04$ & $2.0 \mathrm{E}-05$ \\
20 & $2.1 \mathrm{E}-01$ & $4.2 \mathrm{E}-04$ & $2.1 \mathrm{E}-05$ \\
25 & $2.1 \mathrm{E}-01$ & $4.2 \mathrm{E}-04$ & $2.1 \mathrm{E}-05$ \\
30 & $2.1 \mathrm{E}-01$ & $4.3 \mathrm{E}-04$ & $2.1 \mathrm{E}-05$ \\
62 & $2.2 \mathrm{E}-01$ & $4.7 \mathrm{E}-04$ & $2.2 \mathrm{E}-05$ \\
125 & $2.4 \mathrm{E}-01$ & $5.2 \mathrm{E}-04$ & $2.4 \mathrm{E}-05$ \\
575 & $2.7 \mathrm{E}-01$ & $6.0 \mathrm{E}-04$ & $2.7 \mathrm{E}-05$ \\
750 & $2.7 \mathrm{E}-01$ & $6.0 \mathrm{E}-04$ & $2.7 \mathrm{E}-05$ \\
1000 & $2.7 \mathrm{E}-01$ & $6.0 \mathrm{E}-04$ & $2.7 \mathrm{E}-05$ \\
1025 & $6.3 \mathrm{E}-02$ & $1.8 \mathrm{E}-04$ & $6.8 \mathrm{E}-06$ \\
1050 & $5.3 \mathrm{E}-02$ & $1.5 \mathrm{E}-04$ & $5.7 \mathrm{E}-06$ \\
1100 & $3.8 \mathrm{E}-02$ & $1.1 \mathrm{E}-04$ & $4.0 \mathrm{E}-06$ \\
1200 & $1.9 \mathrm{E}-02$ & $5.4 \mathrm{E}-05$ & $2.0 \mathrm{E}-06$ \\
1400 & $4.7 \mathrm{E}-03$ & $1.3 \mathrm{E}-05$ & $5.0 \mathrm{E}-07$ \\
1600 & $1.2 \mathrm{E}-03$ & $3.4 \mathrm{E}-06$ & $1.3 \mathrm{E}-07$ \\
2000 & $7.3 \mathrm{E}-05$ & $2.1 \mathrm{E}-07$ & $7.9 \mathrm{E}-09$ \\
2500 & $2.3 \mathrm{E}-06$ & $6.6 \mathrm{E}-09$ & $2.5 \mathrm{E}-10$ \\
3000 & $7.2 \mathrm{E}-08$ & $2.1 \mathrm{E}-10$ & $7.7 \mathrm{E}-12$ \\
& & &
\end{tabular}

ABBRM Cancer Risk from Beef Ingestion in Zone $B$ Due to Atmospheric Release

\begin{tabular}{rrrr}
\hline Time $(y r)$ & As & $\mathrm{Cr}$ & $\mathrm{Ni}$ \\
\hline 1 & $3.4 \mathrm{E}-03$ & $0.0 \mathrm{E}+00$ & $0.0 \mathrm{E}+00$ \\
20 & $3.6 \mathrm{E}-03$ & $0.0 \mathrm{E}+00$ & $0.0 \mathrm{E}+00$ \\
25 & $3.6 \mathrm{E}-03$ & $0.0 \mathrm{E}+00$ & $0.0 \mathrm{E}+00$ \\
30 & $3.7 \mathrm{E}-03$ & $0.0 \mathrm{E}+00$ & $0.0 \mathrm{E}+00$ \\
62 & $3.9 \mathrm{E}-03$ & $0.0 \mathrm{E}+00$ & $0.0 \mathrm{E}+00$ \\
125 & $4.2 \mathrm{E}-03$ & $0.0 \mathrm{E}+00$ & $0.0 \mathrm{E}+00$ \\
575 & $4.7 \mathrm{E}-03$ & $0.0 \mathrm{E}+00$ & $0.0 \mathrm{E}+00$ \\
750 & $4.7 \mathrm{E}-03$ & $0.0 \mathrm{E}+00$ & $0.0 \mathrm{E}+00$ \\
1000 & $4.7 \mathrm{E}-03$ & $0.0 \mathrm{E}+00$ & $0.0 \mathrm{E}+00$ \\
1025 & $1.1 \mathrm{E}-03$ & $0.0 \mathrm{E}+00$ & $0.0 \mathrm{E}+00$ \\
1050 & $9.3 \mathrm{E}-04$ & $0.0 \mathrm{E}+00$ & $0.0 \mathrm{E}+00$ \\
1100 & $6.6 \mathrm{E}-04$ & $0.0 \mathrm{E}+00$ & $0.0 \mathrm{E}+00$ \\
1200 & $3.3 \mathrm{E}-04$ & $0.0 \mathrm{E}+00$ & $0.0 \mathrm{E}+00$ \\
1400 & $8.2 \mathrm{E}-05$ & $0.0 \mathrm{E}+00$ & $0.0 \mathrm{E}+00$ \\
1600 & $2.1 \mathrm{E}-05$ & $0.0 \mathrm{E}+00$ & $0.0 \mathrm{E}+00$ \\
2000 & $1.3 \mathrm{E}-06$ & $0.0 \mathrm{E}+00$ & $0.0 \mathrm{E}+00$ \\
2500 & $4.0 \mathrm{E}-08$ & $0.0 \mathrm{E}+00$ & $0.0 \mathrm{E}+00$ \\
3000 & $1.3 \mathrm{E}-09$ & $0.0 \mathrm{E}+00$ & $0.0 \mathrm{E}+00$ \\
& & & \\
\hline
\end{tabular}


GABCU Concentration in Beef $\left(\mathrm{Bq} \mathrm{kg}^{-1}\right)$ in zone $\mathrm{A}$ Due to Groundwater Release

\begin{tabular}{|c|c|c|c|c|c|}
\hline Time (yr) & $U-238 \& 4$ & $\mathrm{Th}-230$ & $\mathrm{Ra}-226$ & $\mathrm{~Pb}-210$ & Po-210 \\
\hline 15 & $0.0 \mathrm{E}+00$ & $0.0 E+00$ & $0.0 E+00$ & $0.0 E+00$ & $0.0 \mathrm{E}+00$ \\
\hline 20 & $0.0 \mathrm{E}+00$ & $0.0 \mathrm{E}+00$ & $0.0 \mathrm{E}+00$ & $0.0 E+00$ & $0.0 \mathrm{E}+00$ \\
\hline 25 & $0.0 \mathrm{E}+00$ & $0.0 E+00$ & $0.0 \mathrm{E}+00$ & $0.0 E+00$ & $0.0 E+00$ \\
\hline 30 & $0.0 \mathrm{E}+00$ & $0.0 \mathrm{E}+00$ & $0.0 \mathrm{E}+00$ & $0.0 E+00$ & $0.0 \mathrm{E}+00$ \\
\hline 60 & $0.0 \mathrm{E}+00$ & $0.0 \mathrm{E}+00$ & $0.0 E+00$ & $0.0 \mathrm{E}+00$ & $0.0 \mathrm{E}+00$ \\
\hline 62 & 1. $4 \mathrm{E}+00$ & $7.2 \mathrm{E}-11$ & $2.1 E-11$ & $7.8 \mathrm{E}-11$ & 2. $0 \mathrm{E}-08$ \\
\hline 80 & $7.5 E+01$ & $2.2 \mathrm{E}-07$ & $1.7 \mathrm{E}-08$ & $6.2 \mathrm{E}-08$ & $1.6 \mathrm{E}-05$ \\
\hline 100 & 1. $6 \mathrm{E}+02$ & $9.6 \mathrm{E}-07$ & 1. $5 E-07$ & $5.5 E-07$ & $1.4 \mathrm{E}-04$ \\
\hline 125 & $2.5 E+02$ & $2.6 \mathrm{E}-06$ & $6.6 \mathrm{E}-07$ & $2.5 \mathrm{E}-06$ & $6.2 E-04$ \\
\hline 575 & $2.5 E+02$ & $3.9 E-05$ & 1. $0 \mathrm{E}-04$ & $3.9 E-04$ & $9.7 E-02$ \\
\hline 590 & $2.5 E+02$ & $4.0 \mathrm{E}-05$ & $1.9 E-02$ & $7.6 \mathrm{E}-02$ & 1. $9 E \div 01$ \\
\hline 620 & $2.5 E+02$ & $4.2 \mathrm{E}-05$ & $1.8 \mathrm{E}-01$ & $7.5 E-01$ & $1.9 \mathrm{E}+02$ \\
\hline 750 & $2.5 E+02$ & $5.3 E-05$ & $8.8 \mathrm{E}-01$ & 3. $6 E+00$ & $8.9 E+02$ \\
\hline 1000 & $2.5 E+02$ & $7.3 E-05$ & $2.1 \mathrm{E}+00$ & $8.6 E+00$ & $2.1 E+03$ \\
\hline 1015 & $2.5 E+02$ & $7.5 E-05$ & $2.2 E+00$ & $8.9 E+00$ & $2.2 \mathrm{E}+03$ \\
\hline 1025 & $2.5 \mathrm{E}+02$ & $7.5 \mathrm{E}-05$ & $2.2 \mathrm{E}+00$ & $9.0 \mathrm{E}+00$ & $2.3 E+03$ \\
\hline 1060 & $2.5 \mathrm{E}+02$ & $7.8 \mathrm{E}-05$ & $2.4 \mathrm{E}+00$ & $9.7 \mathrm{E}+00$ & $2.4 E+03$ \\
\hline 1080 & $2.5 E+02$ & $8.0 E-05$ & $2.5 E+00$ & 1. $0 E+01$ & $2.5 E+03$ \\
\hline 1120 & 1. $4 \mathrm{E}+01$ & $8.1 \mathrm{E}-05$ & $2.7 E+00$ & 1. $1 E+01$ & $2.7 E+03$ \\
\hline 1175 & $0.0 \mathrm{E}+00$ & $8.1 E-05$ & $2.9 E+00$ & 1. $2 \mathrm{E}+01$ & $2.9 \mathrm{E}+03$ \\
\hline 1575 & $0.0 E+00$ & $7.9 \mathrm{E}-05$ & $2 \cdot 9 E+00$ & $1.2 E+01$ & $3.0 E+03$ \\
\hline 1750 & $0.0 E+00$ & 8.0E-05 & $2.1 E+00$ & 8. $4 \mathrm{E}+00$ & $2.1 E+03$ \\
\hline 2175 & $0.0 E+00$ & $7.8 \mathrm{E}-05$ & $7.0 \mathrm{E}-02$ & 3. $0 \mathrm{E}-01$ & $7.0 E+01$ \\
\hline 4000 & $0.0 E+00$ & $7.6 \mathrm{E}-05$ & $7.0 E-02$ & 3. $0 \mathrm{E}-01$ & $7.0 E+01$ \\
\hline 6000 & $0.0 \mathrm{E}+00$ & $7.5 \mathrm{E}-05$ & $7.0 \mathrm{E}-02$ & 3. $0 E-01$ & 8. $0 E+01$ \\
\hline 8000 & $0.0 E+00$ & $7.4 \mathrm{E}-05$ & $7.0 \mathrm{E}-02$ & 3. $0 E-01$ & 7. $0 \mathrm{E}+01$ \\
\hline 10000 & $0.0 \mathrm{E}+00$ & $7.3 \mathrm{E}-05$ & $8.0 \mathrm{E}-02$ & 3. $0 \mathrm{E}-01$ & $7.0 \mathrm{E}+01$ \\
\hline
\end{tabular}


GBBCU Concentration in Beef ( $\mathrm{Bq} \mathrm{kg}^{-1}$ ) in Zone $\mathrm{B}$ Due to Groundwater Release

\begin{tabular}{|c|c|c|c|c|c|}
\hline Time (yr) & $U-238 \& 4$ & Th -230 & $R a-226$ & $\mathrm{~Pb}-210$ & Po- 210 \\
\hline 15 & $0.0 \mathrm{E}+00$ & $0.0 E+00$ & $0.0 E+00$ & $0.0 E+00$ & $0.0 \mathrm{E}+00$ \\
\hline 20 & $0.0 E+00$ & $0.0 \mathrm{E}+00$ & $0.0 E+00$ & $0.0 E+00$ & $0.0 E+00$ \\
\hline 25 & $0.0 E+00$ & $0.0 E+00$ & $0.0 E+00$ & $0.0 \mathrm{E}+00$ & $0.0 \mathrm{E}+00$ \\
\hline 30 & $0.0 E+00$ & $0.0 \mathrm{E}+00$ & $0.0 E+00$ & $0.0 E+00$ & $0.0 E+00$ \\
\hline 60 & $0.0 E+00$ & $0.0 E+00$ & $0.0 \mathrm{E}+00$ & $0.0 E+00$ & $0.0 \mathrm{E}+00$ \\
\hline 62 & $1.6 \mathrm{E}-01$ & $8.6 E-12$ & $2.5 E-12$ & $9.3 E-12$ & $2.3 E-09$ \\
\hline 80 & $8.9 \mathrm{E}+00$ & $2.6 E-08$ & $2.0 E-09$ & $7.4 E-09$ & $1.8 \mathrm{E}-06$ \\
\hline 100 & $1.9 E+01$ & 1.1E-07 & $1.7 \mathrm{E}-08$ & $6.5 E-08$ & 1. $6 \mathrm{E}-05$ \\
\hline 125 & $3.0 \mathrm{E}+01$ & $3.1 E-07$ & $7.9 E-08$ & $3.0 \mathrm{E}-07$ & $7.4 E-05$ \\
\hline 575 & 3. $0 E+01$ & $4.6 E-06$ & 1. $2 E-05$ & $4.7 E-05$ & 1. $2 \mathrm{E}-02$ \\
\hline 590 & $3.0 E+01$ & $4.7 E-06$ & $2.2 \mathrm{E}-03$ & $9.0 E-03$ & $2.3 E+00$ \\
\hline 620 & $3.0 E+01$ & $5.0 \mathrm{E}-06$ & $2.2 \mathrm{E}-02$ & $8.9 E-02$ & $2.2 E+01$ \\
\hline 750 & 3. $0 E+01$ & $6.3 E-06$ & 1. $0 \mathrm{E}-01$ & $4.2 \mathrm{E}-01$ & $1.1 E+02$ \\
\hline 1000 & $3.0 \mathrm{E}+01$ & $8.7 E-06$ & $2.5 \mathrm{E}-01$ & 1. $0 \mathrm{E}+00$ & $2.6 \mathrm{E}+02$ \\
\hline 1015 & $3.0 E+01$ & $8.9 E-06$ & 2. $6 E-01$ & 1. $1 \mathrm{E}+00$ & $2.6 \mathrm{E}+02$ \\
\hline 1025 & $3.0 E+01$ & $9.0 E-06$ & $2.7 \mathrm{E}-01$ & $1.1 E+00$ & $2.7 E+02$ \\
\hline 1060 & $3.0 E+01$ & $9.3 E-06$ & $2.8 \mathrm{E}-01$ & 1. $2 \mathrm{E}+00$ & $2.9 E+02$ \\
\hline 1080 & 3. $0 \mathrm{E}+01$ & $9.5 \mathrm{E}-06$ & 3. $0 \mathrm{E}-01$ & 1. $2 E+00$ & 3. $0 E+02$ \\
\hline 1120 & 1. $6 \mathrm{E}+00$ & $9.6 \mathrm{E}-06$ & $3.2 \mathrm{E}-01$ & 1. $3 E+00$ & 3. $2 E+02$ \\
\hline 1175 & $0.0 \mathrm{E}+00$ & $9.6 \mathrm{E}-06$ & 3. $4 E-01$ & 1. $4 \mathrm{E}+00$ & $3.5 E+02$ \\
\hline 1575 & $0.0 \mathrm{E}+00$ & $9.5 E-06$ & $3.5 E-01$ & 1. $4 E+00$ & $3.5 E+02$ \\
\hline 1750 & $0.0 E+00$ & $9.5 E-06$ & 2. $5 E-01$ & 1. $0 E+00$ & $2.5 E+02$ \\
\hline 2175 & $0.0 E+00$ & $9.2 \mathrm{E}-06$ & $8.0 \mathrm{E}-03$ & 3. $0 E-02$ & $8.0 \mathrm{E}+00$ \\
\hline 4000 & $0.0 E+00$ & $9.1 E-06$ & $8.0 E-03$ & $3.0 \mathrm{E}-02$ & $8.0 E+00$ \\
\hline 6000 & $0.0 \mathrm{E}+00$ & $9.0 \mathrm{E}-06$ & $8.0 E-03$ & $3.0 \mathrm{E}-02$ & $9.0 \mathrm{E}+00$ \\
\hline 8000 & $0.0 \mathrm{E}+00$ & $8.7 E-06$ & $9.0 E-03$ & $4.0 \mathrm{E}-02$ & $9.0 E+00$ \\
\hline 10000 & $0.0 \mathrm{E}+00$ & $8.6 E-06$ & $9.0 \mathrm{E}-03$ & $4.0 \mathrm{E}-02$ & $9.0 E+O O$ \\
\hline
\end{tabular}


GABDU Dose from Beef Ingestion in Zone A Due to Groundwater Release ( $\mathrm{SV} \mathrm{yr}^{-1}$ )

\begin{tabular}{rcccccc}
\hline Time $(\mathrm{Yr})$ & $\mathrm{U}-238 \& 4$ & Th-230 & Ra-226 & Pb-210 & Po-210 & Chain \\
\hline 15 & $0.0 \mathrm{E}+00$ & $0.0 \mathrm{E}+00$ & $0.0 \mathrm{E}+00$ & $0.0 \mathrm{E}+00$ & $0.0 \mathrm{E}+00$ & $0.0 \mathrm{E}+00$ \\
20 & $0.0 \mathrm{E}+00$ & $0.0 \mathrm{E}+00$ & $0.0 \mathrm{E}+00$ & $0.0 \mathrm{E}+00$ & $0.0 \mathrm{E}+00$ & $0.0 \mathrm{E}+00$ \\
25 & $0.0 \mathrm{E}+00$ & $0.0 \mathrm{E}+00$ & $0.0 \mathrm{E}+00$ & $0.0 \mathrm{E}+00$ & $0.0 \mathrm{E}+00$ & $0.0 \mathrm{E}+00$ \\
30 & $0.0 \mathrm{E}+00$ & $0.0 \mathrm{E}+00$ & $0.0 \mathrm{E}+00$ & $0.0 \mathrm{E}+00$ & $0.0 \mathrm{E}+00$ & $0.0 \mathrm{E}+00$ \\
60 & $0.0 \mathrm{E}+00$ & $0.0 \mathrm{E}+00$ & $0.0 \mathrm{E}+00$ & $0.0 \mathrm{E}+00$ & $0.0 \mathrm{E}+00$ & $0.0 \mathrm{E}+00$ \\
62 & $2.8 \mathrm{E}-06$ & $1.3 \mathrm{E}-15$ & $2.3 \mathrm{E}-16$ & $5.1 \mathrm{E}-15$ & $6.0 \mathrm{E}-13$ & $2.8 \mathrm{E}-06$ \\
80 & $1.5 \mathrm{E}-04$ & $3.8 \mathrm{E}-12$ & $1.8 \mathrm{E}-13$ & $4.0 \mathrm{E}-12$ & $4.8 \mathrm{E}-10$ & $1.5 \mathrm{E}-04$ \\
100 & $3.2 \mathrm{E}-04$ & $1.7 \mathrm{E}-11$ & $1.6 \mathrm{E}-12$ & $3.6 \mathrm{E}-11$ & $4.2 \mathrm{E}-09$ & $3.2 \mathrm{E}-04$ \\
125 & $5.1 \mathrm{E}-04$ & $4.6 \mathrm{E}-11$ & $7.3 \mathrm{E}-12$ & $1.6 \mathrm{E}-10$ & $1.9 \mathrm{E}-08$ & $5.1 \mathrm{E}-04$ \\
575 & $5.1 \mathrm{E}-04$ & $6.8 \mathrm{E}-10$ & $1.2 \mathrm{E}-09$ & $2.5 \mathrm{E}-08$ & $3.0 \mathrm{E}-06$ & $5.1 \mathrm{E}-04$ \\
590 & $5.1 \mathrm{E}-04$ & $7.0 \mathrm{E}-10$ & $2.1 \mathrm{E}-07$ & $4.9 \mathrm{E}-06$ & $5.9 \mathrm{E}-04$ & $1.1 \mathrm{E}-03$ \\
620 & $5.1 \mathrm{E}-04$ & $7.4 \mathrm{E}-10$ & $2.0 \mathrm{E}-06$ & $4.9 \mathrm{E}-05$ & $5.8 \mathrm{E}-03$ & $6.3 \mathrm{E}-03$ \\
750 & $5.1 \mathrm{E}-04$ & $9.2 \mathrm{E}-10$ & $9.7 \mathrm{E}-06$ & $2.3 \mathrm{E}-04$ & $2.8 \mathrm{E}-02$ & $2.8 \mathrm{E}-02$ \\
1000 & $5.1 \mathrm{E}-04$ & $1.3 \mathrm{E}-09$ & $2.3 \mathrm{E}-05$ & $5.6 \mathrm{E}-04$ & $6.6 \mathrm{E}-02$ & $6.7 \mathrm{E}-02$ \\
1015 & $5.1 \mathrm{E}-04$ & $1.3 \mathrm{E}-09$ & $2.4 \mathrm{E}-05$ & $5.8 \mathrm{E}-04$ & $6.9 \mathrm{E}-02$ & $7.0 \mathrm{E}-02$ \\
1025 & $5.1 \mathrm{E}-04$ & $1.3 \mathrm{E}-09$ & $2.5 \mathrm{E}-05$ & $5.9 \mathrm{E}-04$ & $7.0 \mathrm{E}-02$ & $7.1 \mathrm{E}-02$ \\
1060 & $5.1 \mathrm{E}-04$ & $1.4 \mathrm{E}-09$ & $2.6 \mathrm{E}-05$ & $6.3 \mathrm{E}-04$ & $7.5 \mathrm{E}-02$ & $7.6 \mathrm{E}-02$ \\
1080 & $5.1 \mathrm{E}-04$ & $1.4 \mathrm{E}-09$ & $2.7 \mathrm{E}-05$ & $6.5 \mathrm{E}-04$ & $7.8 \mathrm{E}-02$ & $7.9 \mathrm{E}-02$ \\
1120 & $2.7 \mathrm{E}-05$ & $1.4 \mathrm{E}-09$ & $2.9 \mathrm{E}-05$ & $7.0 \mathrm{E}-04$ & $8.4 \mathrm{E}-02$ & $8.4 \mathrm{E}-02$ \\
1175 & $0.0 \mathrm{E}+00$ & $1.4 \mathrm{E}-09$ & $3.2 \mathrm{E}-05$ & $7.6 \mathrm{E}-04$ & $9.1 \mathrm{E}-02$ & $9.2 \mathrm{E}-02$ \\
1575 & $0.0 \mathrm{E}+00$ & $1.4 \mathrm{E}-09$ & $3.2 \mathrm{E}-05$ & $7.7 \mathrm{E}-04$ & $9.2 \mathrm{E}-02$ & $9.3 \mathrm{E}-02$ \\
1750 & $0.0 \mathrm{E}+00$ & $1.4 \mathrm{E}-09$ & $2.3 \mathrm{E}-05$ & $5.4 \mathrm{E}-04$ & $6.5 \mathrm{E}-02$ & $6.5 \mathrm{E}-02$ \\
2175 & $0.0 \mathrm{E}+00$ & $1.4 \mathrm{E}-09$ & $7.0 \mathrm{E}-07$ & $1.7 \mathrm{E}-05$ & $2.1 \mathrm{E}-03$ & $2.1 \mathrm{E}-03$ \\
4000 & $0.0 \mathrm{E}+00$ & $1.3 \mathrm{E}-09$ & $8.0 \mathrm{E}-07$ & $1.8 \mathrm{E}-05$ & $2.2 \mathrm{E}-03$ & $2.2 \mathrm{E}-03$ \\
6000 & $0.0 \mathrm{E}+00$ & $1.3 \mathrm{E}-09$ & $8.0 \mathrm{E}-07$ & $2.0 \mathrm{E}-05$ & $2.4 \mathrm{E}-03$ & $2.4 \mathrm{E}-03$ \\
8000 & $0.0 \mathrm{E}+00$ & $1.3 \mathrm{E}-09$ & $8.0 \mathrm{E}-07$ & $1.9 \mathrm{E}-05$ & $2.1 \mathrm{E}-03$ & $2.1 \mathrm{E}-03$ \\
10000 & $0.0 \mathrm{E}+00$ & $1.2 \mathrm{E}-09$ & $8.0 \mathrm{E}-07$ & $2.0 \mathrm{E}-05$ & $2.0 \mathrm{E}-03$ & $2.0 \mathrm{E}-03$ \\
& & & & & & \\
\hline
\end{tabular}


GBBDU Dose from Beef Ingestion in Zone B Due to Groundwater Release (SV $\mathrm{Yr}^{-1}$ )

\begin{tabular}{rcccccc}
\hline Time $(\mathrm{yr})$ & $\mathrm{U}-238 \& 4$ & $\mathrm{Th}-230$ & Ra-226 & Pb-210 & PO-210 & Chain \\
\hline 15 & $0.0 \mathrm{E}+00$ & $0.0 \mathrm{E}+00$ & $0.0 \mathrm{E}+00$ & $0.0 \mathrm{E}+00$ & $0.0 \mathrm{E}+00$ & $0.0 \mathrm{E}+00$ \\
20 & $0.0 \mathrm{E}+00$ & $0.0 \mathrm{E}+00$ & $0.0 \mathrm{E}+00$ & $0.0 \mathrm{E}+00$ & $0.0 \mathrm{E}+00$ & $0.0 \mathrm{E}+00$ \\
25 & $0.0 \mathrm{E}+00$ & $0.0 \mathrm{E}+00$ & $0.0 \mathrm{E}+00$ & $0.0 \mathrm{E}+00$ & $0.0 \mathrm{E}+00$ & $0.0 \mathrm{E}+00$ \\
30 & $0.0 \mathrm{E}+00$ & $0.0 \mathrm{E}+00$ & $0.0 \mathrm{E}+00$ & $0.0 \mathrm{E}+00$ & $0.0 \mathrm{E}+00$ & $0.0 \mathrm{E}+00$ \\
60 & $0.0 \mathrm{E}+00$ & $0.0 \mathrm{E}+00$ & $0.0 \mathrm{E}+00$ & $0.0 \mathrm{E}+00$ & $0.0 \mathrm{E}+00$ & $0.0 \mathrm{E}+00$ \\
62 & $3.3 \mathrm{E}-07$ & $1.5 \mathrm{E}-16$ & $2.7 \mathrm{E}-17$ & $6.0 \mathrm{E}-16$ & $7.2 \mathrm{E}-14$ & $3.3 \mathrm{E}-07$ \\
80 & $1.8 \mathrm{E}-05$ & $4.6 \mathrm{E}-13$ & $2.2 \mathrm{E}-14$ & $4.8 \mathrm{E}-13$ & $5.7 \mathrm{E}-11$ & $1.8 \mathrm{E}-05$ \\
100 & $3.8 \mathrm{E}-05$ & $2.0 \mathrm{E}-12$ & $1.9 \mathrm{E}-13$ & $4.2 \mathrm{E}-12$ & $5.0 \mathrm{E}-10$ & $3.8 \mathrm{E}-05$ \\
125 & $6.1 \mathrm{E}-05$ & $5.4 \mathrm{E}-12$ & $8.7 \mathrm{E}-13$ & $1.9 \mathrm{E}-11$ & $2.3 \mathrm{E}-09$ & $6.1 \mathrm{E}-05$ \\
575 & $6.1 \mathrm{E}-05$ & $8.1 \mathrm{E}-11$ & $1.4 \mathrm{E}-10$ & $3.0 \mathrm{E}-09$ & $3.6 \mathrm{E}-07$ & $6.1 \mathrm{E}-05$ \\
590 & $6.1 \mathrm{E}-05$ & $8.3 \mathrm{E}-11$ & $2.4 \mathrm{E}-08$ & $5.8 \mathrm{E}-07$ & $7.0 \mathrm{E}-05$ & $1.3 \mathrm{E}-04$ \\
620 & $6.1 \mathrm{E}-05$ & $8.8 \mathrm{E}-11$ & $2.4 \mathrm{E}-07$ & $5.8 \mathrm{E}-06$ & $6.9 \mathrm{E}-04$ & $7.6 \mathrm{E}-04$ \\
750 & $6.1 \mathrm{E}-05$ & $1.1 \mathrm{E}-10$ & $1.2 \mathrm{E}-06$ & $2.8 \mathrm{E}-05$ & $3.3 \mathrm{E}-03$ & $3.4 \mathrm{E}-03$ \\
1000 & $6.1 \mathrm{E}-05$ & $1.5 \mathrm{E}-10$ & $2.8 \mathrm{E}-06$ & $6.6 \mathrm{E}-05$ & $7.9 \mathrm{E}-03$ & $8.0 \mathrm{E}-03$ \\
1015 & $6.1 \mathrm{E}-05$ & $1.6 \mathrm{E}-10$ & $2.9 \mathrm{E}-06$ & $6.9 \mathrm{E}-05$ & $8.2 \mathrm{E}-03$ & $8.3 \mathrm{E}-03$ \\
1025 & $6.1 \mathrm{E}-05$ & $1.6 \mathrm{E}-10$ & $2.9 \mathrm{E}-06$ & $7.0 \mathrm{E}-05$ & $8.3 \mathrm{E}-03$ & $8.5 \mathrm{E}-03$ \\
1060 & $6.1 \mathrm{E}-05$ & $1.6 \mathrm{E}-10$ & $3.1 \mathrm{E}-06$ & $7.5 \mathrm{E}-05$ & $8.9 \mathrm{E}-03$ & $9.1 \mathrm{E}-03$ \\
1080 & $6.0 \mathrm{E}-05$ & $1.7 \mathrm{E}-10$ & $3.3 \mathrm{E}-06$ & $7.8 \mathrm{E}-05$ & $9.3 \mathrm{E}-03$ & $9.4 \mathrm{E}-03$ \\
1120 & $3.3 \mathrm{E}-06$ & $1.7 \mathrm{E}-10$ & $3.5 \mathrm{E}-06$ & $8.3 \mathrm{E}-05$ & $1.0 \mathrm{E}-02$ & $1.0 \mathrm{E}-02$ \\
1175 & $0.0 \mathrm{E}+00$ & $1.7 \mathrm{E}-10$ & $3.8 \mathrm{E}-06$ & $9.1 \mathrm{E}-05$ & $1.1 \mathrm{E}-02$ & $1.1 \mathrm{E}-02$ \\
1575 & $0.0 \mathrm{E}+00$ & $1.7 \mathrm{E}-10$ & $3.8 \mathrm{E}-06$ & $9.2 \mathrm{E}-05$ & $1.1 \mathrm{E}-02$ & $1.1 \mathrm{E}-02$ \\
1750 & $0.0 \mathrm{E}+00$ & $1.7 \mathrm{E}-10$ & $2.7 \mathrm{E}-06$ & $6.5 \mathrm{E}-05$ & $7.7 \mathrm{E}-03$ & $7.8 \mathrm{E}-03$ \\
2175 & $0.0 \mathrm{E}+00$ & $1.6 \mathrm{E}-10$ & $9.0 \mathrm{E}-08$ & $2.1 \mathrm{E}-06$ & $3.0 \mathrm{E}-04$ & $3.0 \mathrm{E}-04$ \\
4000 & $0.0 \mathrm{E}+00$ & $1.6 \mathrm{E}-10$ & $8.0 \mathrm{E}-08$ & $2.1 \mathrm{E}-06$ & $3.0 \mathrm{E}-04$ & $3.0 \mathrm{E}-04$ \\
6000 & $0.0 \mathrm{E}+00$ & $1.6 \mathrm{E}-10$ & $9.0 \mathrm{E}-08$ & $2.4 \mathrm{E}-06$ & $3.0 \mathrm{E}-04$ & $3.0 \mathrm{E}-04$ \\
8000 & $0.0 \mathrm{E}+00$ & $1.5 \mathrm{E}-10$ & $9.0 \mathrm{E}-08$ & $2.3 \mathrm{E}-06$ & $2.0 \mathrm{E}-04$ & $2.0 \mathrm{E}-04$ \\
10000 & $0.0 \mathrm{E}+00$ & $1.5 \mathrm{E}-10$ & $1.0 \mathrm{E}-07$ & $2.0 \mathrm{E}-06$ & $3.0 \mathrm{E}-04$ & $3.0 \mathrm{E}-04$ \\
& & & & & & \\
\hline
\end{tabular}


GABRU Cancer Risk from Beef Ingestion in Zone A Due to Groundwater Release

\begin{tabular}{rcccccc}
\hline Time $(Y r)$ & $\mathrm{U}-238 \& 4$ & Th-230 & Ra-226 & Pb-210 & Po-210 & Chain \\
\hline 15 & $0.0 \mathrm{E}+00$ & $0.0 \mathrm{E}+00$ & $0.0 \mathrm{E}+00$ & $0.0 \mathrm{E}+00$ & $0.0 \mathrm{E}+00$ & $0.0 \mathrm{E}+00$ \\
20 & $0.0 \mathrm{E}+00$ & $0.0 \mathrm{E}+00$ & $0.0 \mathrm{E}+00$ & $0.0 \mathrm{E}+00$ & $0.0 \mathrm{E}+00$ & $0.0 \mathrm{E}+00$ \\
25 & $0.0 \mathrm{E}+00$ & $0.0 \mathrm{E}+00$ & $0.0 \mathrm{E}+00$ & $0.0 \mathrm{E}+00$ & $0.0 \mathrm{E}+00$ & $0.0 \mathrm{E}+00$ \\
30 & $0.0 \mathrm{E}+00$ & $0.0 \mathrm{E}+00$ & $0.0 \mathrm{E}+00$ & $0.0 \mathrm{E}+00$ & $0.0 \mathrm{E}+00$ & $0.0 \mathrm{E}+00$ \\
60 & $0.0 \mathrm{E}+00$ & $0.0 \mathrm{E}+00$ & $0.0 \mathrm{E}+00$ & $0.0 \mathrm{E}+00$ & $0.0 \mathrm{E}+00$ & $0.0 \mathrm{E}+00$ \\
62 & $2.8 \mathrm{E}-06$ & $8.8 \mathrm{E}-17$ & $2.3 \mathrm{E}-16$ & $3.8 \mathrm{E}-15$ & $2.8 \mathrm{E}-13$ & $2.8 \mathrm{E}-06$ \\
80 & $1.6 \mathrm{E}-04$ & $2.7 \mathrm{E}-13$ & $1.9 \mathrm{E}-13$ & $3.0 \mathrm{E}-12$ & $2.2 \mathrm{E}-10$ & $1.6 \mathrm{E}-04$ \\
100 & $3.3 \mathrm{E}-04$ & $1.2 \mathrm{E}-12$ & $1.6 \mathrm{E}-12$ & $2.7 \mathrm{E}-11$ & $2.0 \mathrm{E}-09$ & $3.3 \mathrm{E}-04$ \\
125 & $5.2 \mathrm{E}-04$ & $3.2 \mathrm{E}-12$ & $7.4 \mathrm{E}-12$ & $1.2 \mathrm{E}-10$ & $8.9 \mathrm{E}-09$ & $5.2 \mathrm{E}-04$ \\
575 & $5.2 \mathrm{E}-04$ & $4.7 \mathrm{E}-11$ & $1.2 \mathrm{E}-09$ & $1.9 \mathrm{E}-08$ & $1.4 \mathrm{E}-06$ & $5.3 \mathrm{E}-04$ \\
590 & $5.2 \mathrm{E}-04$ & $4.9 \mathrm{E}-11$ & $2.1 \mathrm{E}-07$ & $3.7 \mathrm{E}-06$ & $2.7 \mathrm{E}-04$ & $8.0 \mathrm{E}-04$ \\
620 & $5.2 \mathrm{E}-04$ & $5.2 \mathrm{E}-11$ & $2.1 \mathrm{E}-06$ & $3.7 \mathrm{E}-05$ & $2.7 \mathrm{E}-03$ & $3.2 \mathrm{E}-03$ \\
750 & $5.2 \mathrm{E}-04$ & $6.4 \mathrm{E}-11$ & $9.8 \mathrm{E}-06$ & $1.7 \mathrm{E}-04$ & $1.3 \mathrm{E}-02$ & $1.3 \mathrm{E}-02$ \\
1000 & $5.2 \mathrm{E}-04$ & $9.0 \mathrm{E}-11$ & $2.4 \mathrm{E}-05$ & $4.2 \mathrm{E}-04$ & $3.1 \mathrm{E}-02$ & $3.2 \mathrm{E}-02$ \\
1015 & $5.2 \mathrm{E}-04$ & $9.1 \mathrm{E}-11$ & $2.4 \mathrm{E}-05$ & $4.3 \mathrm{E}-04$ & $3.2 \mathrm{E}-02$ & $3.3 \mathrm{E}-02$ \\
1025 & $5.2 \mathrm{E}-04$ & $9.2 \mathrm{E}-11$ & $2.5 \mathrm{E}-05$ & $4.4 \mathrm{E}-04$ & $3.2 \mathrm{E}-02$ & $3.3 \mathrm{E}-02$ \\
1060 & $5.2 \mathrm{E}-04$ & $9.6 \mathrm{E}-11$ & $2.7 \mathrm{E}-05$ & $4.7 \mathrm{E}-04$ & $3.5 \mathrm{E}-02$ & $3.6 \mathrm{E}-02$ \\
1080 & $5.2 \mathrm{E}-04$ & $9.8 \mathrm{E}-11$ & $2.8 \mathrm{E}-05$ & $4.9 \mathrm{E}-04$ & $3.6 \mathrm{E}-02$ & $3.7 \mathrm{E}-02$ \\
1120 & $2.8 \mathrm{E}-05$ & $9.9 \mathrm{E}-11$ & $3.0 \mathrm{E}-05$ & $5.3 \mathrm{E}-04$ & $3.9 \mathrm{E}-02$ & $3.9 \mathrm{E}-02$ \\
1175 & $1.0 \mathrm{E}-07$ & $9.9 \mathrm{E}-11$ & $3.2 \mathrm{E}-05$ & $5.8 \mathrm{E}-04$ & $4.2 \mathrm{E}-02$ & $4.3 \mathrm{E}-02$ \\
1575 & $0.0 \mathrm{E}+00$ & $9.8 \mathrm{E}-11$ & $3.3 \mathrm{E}-05$ & $5.8 \mathrm{E}-04$ & $4.3 \mathrm{E}-02$ & $4.3 \mathrm{E}-02$ \\
1750 & $0.0 \mathrm{E}+00$ & $9.7 \mathrm{E}-11$ & $2.3 \mathrm{E}-05$ & $4.1 \mathrm{E}-04$ & $3.0 \mathrm{E}-02$ & $3.0 \mathrm{E}-02$ \\
2175 & $1.0 \mathrm{E}-07$ & $9.5 \mathrm{E}-11$ & $7.5 \mathrm{E}-07$ & $1.3 \mathrm{E}-05$ & $9.8 \mathrm{E}-04$ & $9.9 \mathrm{E}-04$ \\
4000 & $1.0 \mathrm{E}-07$ & $9.4 \mathrm{E}-11$ & $7.7 \mathrm{E}-07$ & $1.4 \mathrm{E}-05$ & $1.0 \mathrm{E}-03$ & $1.0 \mathrm{E}-03$ \\
6000 & $1.0 \mathrm{E}-07$ & $9.2 \mathrm{E}-11$ & $7.8 \mathrm{E}-07$ & $1.5 \mathrm{E}-05$ & $1.1 \mathrm{E}-03$ & $1.1 \mathrm{E}-03$ \\
8000 & $0.0 \mathrm{E}+00$ & $9.0 \mathrm{E}-11$ & $8.2 \mathrm{E}-07$ & $1.5 \mathrm{E}-05$ & $1.1 \mathrm{E}-03$ & $1.1 \mathrm{E}-03$ \\
10000 & $1.0 \mathrm{E}-07$ & $8.9 \mathrm{E}-11$ & $8.6 \mathrm{E}-07$ & $1.5 \mathrm{E}-05$ & $1.1 \mathrm{E}-03$ & $1.1 \mathrm{E}-03$ \\
& & & & & & \\
\hline
\end{tabular}


GBBRU Cancer Risk from Beef Ingestion in Zone B Due to Groundwater Release

\begin{tabular}{rllllll}
\hline Time $(y r)$ & $\mathrm{U}-238 \& 4$ & $\mathrm{Th}-230$ & $\mathrm{Ra}-226$ & $\mathrm{~Pb}-210$ & $\mathrm{PO}-210$ & Chain \\
\hline 15 & $0.0 \mathrm{E}+00$ & $0.0 \mathrm{E}+00$ & $0.0 \mathrm{E}+00$ & $0.0 \mathrm{E}+00$ & $0.0 \mathrm{E}+00$ & $0.0 \mathrm{E}+00$ \\
20 & $0.0 \mathrm{E}+00$ & $0.0 \mathrm{E}+00$ & $0.0 \mathrm{E}+00$ & $0.0 \mathrm{E}+00$ & $0.0 \mathrm{E}+00$ & $0.0 \mathrm{E}+00$ \\
25 & $0.0 \mathrm{E}+00$ & $0.0 \mathrm{E}+00$ & $0.0 \mathrm{E}+00$ & $0.0 \mathrm{E}+00$ & $0.0 \mathrm{E}+00$ & $0.0 \mathrm{E}+00$ \\
30 & $0.0 \mathrm{E}+00$ & $0.0 \mathrm{E}+00$ & $0.0 \mathrm{E}+00$ & $0.0 \mathrm{E}+00$ & $0.0 \mathrm{E}+00$ & $0.0 \mathrm{E}+00$ \\
60 & $0.0 \mathrm{E}+00$ & $0.0 \mathrm{E}+00$ & $0.0 \mathrm{E}+00$ & $0.0 \mathrm{E}+00$ & $0.0 \mathrm{E}+00$ & $0.0 \mathrm{E}+00$ \\
62 & $3.4 \mathrm{E}-07$ & $1.1 \mathrm{E}-17$ & $2.8 \mathrm{E}-17$ & $4.5 \mathrm{E}-16$ & $3.3 \mathrm{E}-14$ & $3.4 \mathrm{E}-07$ \\
80 & $1.9 \mathrm{E}-05$ & $3.2 \mathrm{E}-14$ & $2.2 \mathrm{E}-14$ & $3.6 \mathrm{E}-13$ & $2.6 \mathrm{E}-11$ & $1.9 \mathrm{E}-05$ \\
100 & $3.9 \mathrm{E}-05$ & $1.4 \mathrm{E}-13$ & $1.9 \mathrm{E}-13$ & $3.2 \mathrm{E}-12$ & $2.3 \mathrm{E}-10$ & $3.9 \mathrm{E}-05$ \\
125 & $6.2 \mathrm{E}-05$ & $3.8 \mathrm{E}-13$ & $8.8 \mathrm{E}-13$ & $1.4 \mathrm{E}-11$ & $1.1 \mathrm{E}-09$ & $6.2 \mathrm{E}-05$ \\
575 & $6.2 \mathrm{E}-05$ & $5.6 \mathrm{E}-12$ & $1.4 \mathrm{E}-10$ & $2.3 \mathrm{E}-09$ & $1.7 \mathrm{E}-07$ & $6.3 \mathrm{E}-05$ \\
590 & $6.2 \mathrm{E}-05$ & $5.8 \mathrm{E}-12$ & $2.5 \mathrm{E}-08$ & $4.4 \mathrm{E}-07$ & $3.2 \mathrm{E}-05$ & $9.5 \mathrm{E}-05$ \\
620 & $6.2 \mathrm{E}-05$ & $6.1 \mathrm{E}-12$ & $2.5 \mathrm{E}-07$ & $4.4 \mathrm{E}-06$ & $3.2 \mathrm{E}-04$ & $3.9 \mathrm{E}-04$ \\
750 & $6.2 \mathrm{E}-05$ & $7.7 \mathrm{E}-12$ & $1.2 \mathrm{E}-06$ & $2.1 \mathrm{E}-05$ & $1.5 \mathrm{E}-03$ & $1.6 \mathrm{E}-03$ \\
1000 & $6.2 \mathrm{E}-05$ & $1.1 \mathrm{E}-11$ & $2.8 \mathrm{E}-06$ & $5.0 \mathrm{E}-05$ & $3.7 \mathrm{E}-03$ & $3.8 \mathrm{E}-03$ \\
1015 & $6.2 \mathrm{E}-05$ & $1.1 \mathrm{E}-11$ & $2.9 \mathrm{E}-06$ & $5.2 \mathrm{E}-05$ & $3.8 \mathrm{E}-03$ & $3.9 \mathrm{E}-03$ \\
1025 & $6.2 \mathrm{E}-05$ & $1.1 \mathrm{E}-11$ & $3.0 \mathrm{E}-06$ & $5.3 \mathrm{E}-05$ & $3.9 \mathrm{E}-03$ & $4.0 \mathrm{E}-03$ \\
1060 & $6.2 \mathrm{E}-05$ & $1.1 \mathrm{E}-11$ & $3.2 \mathrm{E}-06$ & $5.7 \mathrm{E}-05$ & $4.1 \mathrm{E}-03$ & $4.3 \mathrm{E}-03$ \\
1080 & $6.2 \mathrm{E}-05$ & $1.2 \mathrm{E}-11$ & $3.3 \mathrm{E}-06$ & $5.9 \mathrm{E}-05$ & $4.3 \mathrm{E}-03$ & $4.4 \mathrm{E}-03$ \\
1120 & $3.4 \mathrm{E}-06$ & $1.2 \mathrm{E}-11$ & $3.5 \mathrm{E}-06$ & $6.3 \mathrm{E}-05$ & $4.6 \mathrm{E}-03$ & $4.7 \mathrm{E}-03$ \\
1175 & $0.0 \mathrm{E}+00$ & $1.2 \mathrm{E}-11$ & $3.9 \mathrm{E}-06$ & $6.9 \mathrm{E}-05$ & $5.0 \mathrm{E}-03$ & $5.1 \mathrm{E}-03$ \\
1575 & $0.0 \mathrm{E}+00$ & $1.2 \mathrm{E}-11$ & $3.9 \mathrm{E}-06$ & $6.9 \mathrm{E}-05$ & $5.1 \mathrm{E}-03$ & $5.1 \mathrm{E}-03$ \\
1750 & $0.0 \mathrm{E}+00$ & $1.2 \mathrm{E}-11$ & $2.7 \mathrm{E}-06$ & $4.9 \mathrm{E}-05$ & $3.6 \mathrm{E}-03$ & $3.6 \mathrm{E}-03$ \\
2175 & $0.0 \mathrm{E}+00$ & $1.1 \mathrm{E}-11$ & $8.9 \mathrm{E}-08$ & $1.6 \mathrm{E}-06$ & $1.2 \mathrm{E}-04$ & $1.2 \mathrm{E}-04$ \\
4000 & $0.0 \mathrm{E}+00$ & $1.1 \mathrm{E}-11$ & $9.0 \mathrm{E}-08$ & $1.6 \mathrm{E}-06$ & $1.2 \mathrm{E}-04$ & $1.2 \mathrm{E}-04$ \\
6000 & $0.0 \mathrm{E}+00$ & $1.1 \mathrm{E}-11$ & $9.3 \mathrm{E}-08$ & $1.8 \mathrm{E}-06$ & $1.3 \mathrm{E}-04$ & $1.3 \mathrm{E}-04$ \\
8000 & $0.0 \mathrm{E}+00$ & $1.1 \mathrm{E}-11$ & $9.7 \mathrm{E}-08$ & $1.7 \mathrm{E}-06$ & $1.3 \mathrm{E}-04$ & $1.3 \mathrm{E}-04$ \\
10000 & $0.0 \mathrm{E}+00$ & $1.1 \mathrm{E}-11$ & $1.0 \mathrm{E}-07$ & $1.8 \mathrm{E}-06$ & $1.3 \mathrm{E}-04$ & $1.3 \mathrm{E}-04$ \\
& & & & & & \\
\hline
\end{tabular}




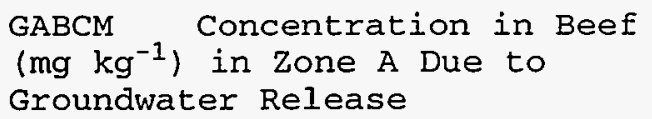

\begin{tabular}{|c|c|c|c|}
\hline Time (yr) & As & $\mathrm{Cr}$ & $\mathrm{Ni}$ \\
\hline 15 & $0.0 \mathrm{E}+00$ & $1.3 E-05$ & $6.4 \mathrm{E}-07$ \\
\hline 20 & $0.0 E+00$ & 1. $0 \mathrm{E}+00$ & 5. $0 \mathrm{E}-02$ \\
\hline 25 & $0.0 \mathrm{E}+00$ & $2.0 E+00$ & 1. $0 \mathrm{E}-01$ \\
\hline 30 & $0.0 \mathrm{E}+00$ & $3.0 E+00$ & $1.5 \mathrm{E}-01$ \\
\hline 60 & $0.0 E+00$ & $3.0 E+00$ & 1. $5 \mathrm{E}-01$ \\
\hline 62 & $0.0 E+00$ & $3.0 E+00$ & 1. $5 \mathrm{E}-01$ \\
\hline 80 & $0.0 \mathrm{E}+00$ & $3.0 E+00$ & 1. $5 \mathrm{E}-01$ \\
\hline 100 & $0.0 \mathrm{E}+00$ & $3.0 E+00$ & 1. $5 \mathrm{E}-01$ \\
\hline 125 & $0.0 E+00$ & $3.0 E+00$ & 1. $5 \mathrm{E}-01$ \\
\hline 575 & $0.0 \mathrm{E}+00$ & $3.0 \mathrm{E}+00$ & $1.5 \mathrm{E}-01$ \\
\hline 590 & $0.0 \mathrm{E}+00$ & $3.0 E+00$ & 1. $5 E-01$ \\
\hline 620 & $0.0 \mathrm{E}+00$ & $3.0 E+00$ & $1.5 E-01$ \\
\hline 750 & $0.0 \mathrm{E}+00$ & $3.0 \mathrm{E}+00$ & 1. $5 \mathrm{E}-01$ \\
\hline 1000 & $0.0 \mathrm{E}+00$ & 3. $0 E+00$ & 1. $5 \mathrm{E}-01$ \\
\hline 1015 & $0.0 \mathrm{E}+00$ & $3.0 E+00$ & $1.5 \mathrm{E}-01$ \\
\hline 1025 & $0.0 \mathrm{E}+00$ & $9.9 E-01$ & $5.0 E-02$ \\
\hline 1060 & $0.0 \mathrm{E}+00$ & $0.0 E+00$ & $0.0 E+00$ \\
\hline 1080 & $0.0 \mathrm{E}+00$ & $0.0 E+00$ & $0.0 E+00$ \\
\hline 1120 & $0.0 \mathrm{E}+00$ & $0.0 \mathrm{E}+00$ & $0.0 E+00$ \\
\hline 1175 & $0.0 \mathrm{E}+00$ & $0.0 E+00$ & $0.0 E+00$ \\
\hline 1575 & $0.0 E+00$ & $0.0 E+00$ & $0.0 E+00$ \\
\hline 1750 & $0.0 \mathrm{E}+00$ & $0.0 \mathrm{E}+00$ & $0.0 E+00$ \\
\hline 2175 & $0.0 \mathrm{E}+00$ & $0.0 E+00$ & $0.0 E+00$ \\
\hline 4000 & $0.0 \mathrm{E}+00$ & $0.0 \mathrm{E}+00$ & $0.0 E+00$ \\
\hline 6000 & $8.4 \mathrm{E}-02$ & $0.0 E+00$ & $0.0 \mathrm{E}+00$ \\
\hline 8000 & $5.1 \mathrm{E}-01$ & $0.0 \mathrm{E}+00$ & $0.0 \mathrm{E}+00$ \\
\hline 10000 & $5.1 E-01$ & $0.0 E+00$ & $0.0 E+00$ \\
\hline
\end{tabular}

GBBCM Concentration in Beef

( $\mathrm{mg} \mathrm{kg}^{-1}$ ) in Zone $\mathrm{B}$ Due to Groundwater Release

\begin{tabular}{rrrr}
\hline Time (yr) & As & $\mathrm{Cr}$ & $\mathrm{Ni}$ \\
\hline 15 & $0.0 \mathrm{E}+00$ & $1.5 \mathrm{E}-06$ & $7.6 \mathrm{E}-08$ \\
20 & $0.0 \mathrm{E}+00$ & $1.2 \mathrm{E}-01$ & $6.0 \mathrm{E}-03$ \\
25 & $0.0 \mathrm{E}+00$ & $2.4 \mathrm{E}-01$ & $1.2 \mathrm{E}-02$ \\
30 & $0.0 \mathrm{E}+00$ & $3.6 \mathrm{E}-01$ & $1.8 \mathrm{E}-02$ \\
60 & $0.0 \mathrm{E}+00$ & $3.6 \mathrm{E}-01$ & $1.8 \mathrm{E}-02$ \\
62 & $0.0 \mathrm{E}+00$ & $3.6 \mathrm{E}-01$ & $1.8 \mathrm{E}-02$ \\
80 & $0.0 \mathrm{E}+00$ & $3.6 \mathrm{E}-01$ & $1.8 \mathrm{E}-02$ \\
100 & $0.0 \mathrm{E}+00$ & $3.6 \mathrm{E}-01$ & $1.8 \mathrm{E}-02$ \\
125 & $0.0 \mathrm{E}+00$ & $3.6 \mathrm{E}-01$ & $1.8 \mathrm{E}-02$ \\
575 & $0.0 \mathrm{E}+00$ & $3.6 \mathrm{E}-01$ & $1.8 \mathrm{E}-02$ \\
590 & $0.0 \mathrm{E}+00$ & $3.6 \mathrm{E}-01$ & $1.8 \mathrm{E}-02$ \\
620 & $0.0 \mathrm{E}+00$ & $3.6 \mathrm{E}-01$ & $1.8 \mathrm{E}-02$ \\
750 & $0.0 \mathrm{E}+00$ & $3.6 \mathrm{E}-01$ & $1.8 \mathrm{E}-02$ \\
1000 & $0.0 \mathrm{E}+00$ & $3.5 \mathrm{E}-01$ & $1.8 \mathrm{E}-02$ \\
1015 & $0.0 \mathrm{E}+00$ & $3.5 \mathrm{E}-01$ & $1.8 \mathrm{E}-02$ \\
1025 & $0.0 \mathrm{E}+00$ & $1.2 \mathrm{E}-01$ & $5.9 \mathrm{E}-03$ \\
1060 & $0.0 \mathrm{E}+00$ & $0.0 \mathrm{E}+00$ & $0.0 \mathrm{E}+00$ \\
1080 & $0.0 \mathrm{E}+00$ & $0.0 \mathrm{E}+00$ & $0.0 \mathrm{E}+00$ \\
1120 & $0.0 \mathrm{E}+00$ & $0.0 \mathrm{E}+00$ & $0.0 \mathrm{E}+00$ \\
1175 & $0.0 \mathrm{E}+00$ & $0.0 \mathrm{E}+00$ & $0.0 \mathrm{E}+00$ \\
1575 & $0.0 \mathrm{E}+00$ & $0.0 \mathrm{E}+00$ & $0.0 \mathrm{E}+00$ \\
1750 & $0.0 \mathrm{E}+00$ & $0.0 \mathrm{E}+00$ & $0.0 \mathrm{E}+00$ \\
2175 & $0.0 \mathrm{E}+00$ & $0.0 \mathrm{E}+00$ & $0.0 \mathrm{E}+00$ \\
4000 & $0.0 \mathrm{E}+00$ & $0.0 \mathrm{E}+00$ & $0.0 \mathrm{E}+00$ \\
6000 & $1.0 \mathrm{E}-02$ & $0.0 \mathrm{E}+00$ & $0.0 \mathrm{E}+00$ \\
8000 & $6.1 \mathrm{E}-02$ & $0.0 \mathrm{E}+00$ & $0.0 \mathrm{E}+00$ \\
10000 & $6.2 \mathrm{E}-02$ & $0.0 \mathrm{E}+00$ & $0.0 \mathrm{E}+00$ \\
& & & \\
\hline & & & \\
\hline
\end{tabular}


GABIM Intake from Beef

Ingestion in Zone A Due to Groundwater Release ( $m g \mathrm{yr}^{-1}$ )

\begin{tabular}{rrrr}
\hline Time $(Y r)$ & As & $\mathrm{Cr}$ & $\mathrm{Ni}$ \\
\hline 15 & $0.0 \mathrm{E}+00$ & $6.4 \mathrm{E}-04$ & $3.2 \mathrm{E}-05$ \\
20 & $0.0 \mathrm{E}+00$ & $5.0 \mathrm{E}+01$ & $2.5 \mathrm{E}+00$ \\
25 & $0.0 \mathrm{E}+00$ & $1.0 \mathrm{E}+02$ & $5.0 \mathrm{E}+00$ \\
30 & $0.0 \mathrm{E}+00$ & $1.5 \mathrm{E}+02$ & $7.5 \mathrm{E}+00$ \\
60 & $0.0 \mathrm{E}+00$ & $1.5 \mathrm{E}+02$ & $7.5 \mathrm{E}+00$ \\
62 & $0.0 \mathrm{E}+00$ & $1.5 \mathrm{E}+02$ & $7.5 \mathrm{E}+00$ \\
80 & $0.0 \mathrm{E}+00$ & $1.5 \mathrm{E}+02$ & $7.5 \mathrm{E}+00$ \\
100 & $0.0 \mathrm{E}+00$ & $1.5 \mathrm{E}+02$ & $7.5 \mathrm{E}+00$ \\
125 & $0.0 \mathrm{E}+00$ & $1.5 \mathrm{E}+02$ & $7.5 \mathrm{E}+00$ \\
575 & $0.0 \mathrm{E}+00$ & $1.5 \mathrm{E}+02$ & $7.5 \mathrm{E}+00$ \\
590 & $0.0 \mathrm{E}+00$ & $1.5 \mathrm{E}+02$ & $7.5 \mathrm{E}+00$ \\
620 & $0.0 \mathrm{E}+00$ & $1.5 \mathrm{E}+02$ & $7.5 \mathrm{E}+00$ \\
750 & $0.0 \mathrm{E}+00$ & $1.5 \mathrm{E}+02$ & $7.5 \mathrm{E}+00$ \\
1000 & $0.0 \mathrm{E}+00$ & $1.5 \mathrm{E}+02$ & $7.4 \mathrm{E}+00$ \\
1015 & $0.0 \mathrm{E}+00$ & $1.5 \mathrm{E}+02$ & $7.4 \mathrm{E}+00$ \\
1025 & $0.0 \mathrm{E}+00$ & $5.0 \mathrm{E}+01$ & $2.5 \mathrm{E}+00$ \\
1060 & $0.0 \mathrm{E}+00$ & $0.0 \mathrm{E}+00$ & $0.0 \mathrm{E}+00$ \\
1080 & $0.0 \mathrm{E}+00$ & $0.0 \mathrm{E}+00$ & $0.0 \mathrm{E}+00$ \\
1120 & $0.0 \mathrm{E}+00$ & $0.0 \mathrm{E}+00$ & $0.0 \mathrm{E}+00$ \\
1175 & $0.0 \mathrm{E}+00$ & $0.0 \mathrm{E}+00$ & $0.0 \mathrm{E}+00$ \\
1575 & $0.0 \mathrm{E}+00$ & $0.0 \mathrm{E}+00$ & $0.0 \mathrm{E}+00$ \\
1750 & $0.0 \mathrm{E}+00$ & $0.0 \mathrm{E}+00$ & $0.0 \mathrm{E}+00$ \\
2175 & $0.0 \mathrm{E}+00$ & $0.0 \mathrm{E}+00$ & $0.0 \mathrm{E}+00$ \\
4000 & $0.0 \mathrm{E}+00$ & $0.0 \mathrm{E}+00$ & $0.0 \mathrm{E}+00$ \\
6000 & $4.2 \mathrm{E}+00$ & $0.0 \mathrm{E}+00$ & $0.0 \mathrm{E}+00$ \\
8000 & $2.6 \mathrm{E}+01$ & $0.0 \mathrm{E}+00$ & $0.0 \mathrm{E}+00$ \\
10000 & $2.6 \mathrm{E}+01$ & $0.0 \mathrm{E}+00$ & $0.0 \mathrm{E}+00$ \\
& & & \\
\hline & & & \\
& & & \\
1000 & &
\end{tabular}

GBBIM Intake from Beef

Ingestion in Zone $B$ Due to Groundwater Release (mg $\mathrm{Yr}^{-1}$ )

\begin{tabular}{rrrr}
\hline Time $(Y r)$ & As & Cr & Ni \\
\hline 15 & $0.0 \mathrm{E}+00$ & $7.6 \mathrm{E}-05$ & $3.8 \mathrm{E}-06$ \\
20 & $0.0 \mathrm{E}+00$ & $5.9 \mathrm{E}+00$ & $3.0 \mathrm{E}-01$ \\
25 & $0.0 \mathrm{E}+00$ & $1.2 \mathrm{E}+01$ & $5.9 \mathrm{E}-01$ \\
30 & $0.0 \mathrm{E}+00$ & $1.8 \mathrm{E}+01$ & $8.9 \mathrm{E}-01$ \\
60 & $0.0 \mathrm{E}+00$ & $1.8 \mathrm{E}+01$ & $8.9 \mathrm{E}-01$ \\
62 & $0.0 \mathrm{E}+00$ & $1.8 \mathrm{E}+01$ & $8.9 \mathrm{E}-01$ \\
80 & $0.0 \mathrm{E}+00$ & $1.8 \mathrm{E}+01$ & $8.9 \mathrm{E}-01$ \\
100 & $0.0 \mathrm{E}+00$ & $1.8 \mathrm{E}+01$ & $8.9 \mathrm{E}-01$ \\
125 & $0.0 \mathrm{E}+00$ & $1.8 \mathrm{E}+01$ & $8.9 \mathrm{E}-01$ \\
575 & $0.0 \mathrm{E}+00$ & $1.8 \mathrm{E}+01$ & $8.9 \mathrm{E}-01$ \\
590 & $0.0 \mathrm{E}+00$ & $1.8 \mathrm{E}+01$ & $8.9 \mathrm{E}-01$ \\
620 & $0.0 \mathrm{E}+00$ & $1.8 \mathrm{E}+01$ & $8.9 \mathrm{E}-01$ \\
750 & $0.0 \mathrm{E}+00$ & $1.8 \mathrm{E}+01$ & $8.9 \mathrm{E}-01$ \\
1000 & $0.0 \mathrm{E}+00$ & $1.8 \mathrm{E}+01$ & $8.9 \mathrm{E}-01$ \\
1015 & $0.0 \mathrm{E}+00$ & $1.8 \mathrm{E}+01$ & $8.9 \mathrm{E}-01$ \\
1025 & $0.0 \mathrm{E}+00$ & $5.9 \mathrm{E}+00$ & $3.0 \mathrm{E}-01$ \\
1060 & $0.0 \mathrm{E}+00$ & $0.0 \mathrm{E}+00$ & $0.0 \mathrm{E}+00$ \\
1080 & $0.0 \mathrm{E}+00$ & $0.0 \mathrm{E}+00$ & $0.0 \mathrm{E}+00$ \\
1120 & $0.0 \mathrm{E}+00$ & $0.0 \mathrm{E}+00$ & $0.0 \mathrm{E}+00$ \\
1175 & $0.0 \mathrm{E}+00$ & $0.0 \mathrm{E}+00$ & $0.0 \mathrm{E}+00$ \\
1575 & $0.0 \mathrm{E}+00$ & $0.0 \mathrm{E}+00$ & $0.0 \mathrm{E}+00$ \\
1750 & $0.0 \mathrm{E}+00$ & $0.0 \mathrm{E}+00$ & $0.0 \mathrm{E}+00$ \\
2175 & $0.0 \mathrm{E}+00$ & $0.0 \mathrm{E}+00$ & $0.0 \mathrm{E}+00$ \\
4000 & $0.0 \mathrm{E}+00$ & $0.0 \mathrm{E}+00$ & $0.0 \mathrm{E}+00$ \\
6000 & $5.0 \mathrm{E}-01$ & $0.0 \mathrm{E}+00$ & $0.0 \mathrm{E}+00$ \\
8000 & $3.1 \mathrm{E}+00$ & $0.0 \mathrm{E}+00$ & $0.0 \mathrm{E}+00$ \\
10000 & $3.1 \mathrm{E}+00$ & $0.0 \mathrm{E}+00$ & $0.0 \mathrm{E}+00$ \\
& & & \\
\hline & & & \\
1000 & &
\end{tabular}


GABRM Cancer Risk from Beef Ingestion in Zone $A$ Due to Groundwater Release

\begin{tabular}{rrrr}
\hline Time $(y r)$ & As & $\mathrm{Cr}$ & $\mathrm{Ni}$ \\
\hline 15 & $0.0 \mathrm{E}+00$ & $0.0 \mathrm{E}+00$ & $0.0 \mathrm{E}+00$ \\
20 & $0.0 \mathrm{E}+00$ & $0.0 \mathrm{E}+00$ & $0.0 \mathrm{E}+00$ \\
25 & $0.0 \mathrm{E}+00$ & $0.0 \mathrm{E}+00$ & $0.0 \mathrm{E}+00$ \\
30 & $0.0 \mathrm{E}+00$ & $0.0 \mathrm{E}+00$ & $0.0 \mathrm{E}+00$ \\
60 & $0.0 \mathrm{E}+00$ & $0.0 \mathrm{E}+00$ & $0.0 \mathrm{E}+00$ \\
62 & $0.0 \mathrm{E}+00$ & $0.0 \mathrm{E}+00$ & $0.0 \mathrm{E}+00$ \\
80 & $0.0 \mathrm{E}+00$ & $0.0 \mathrm{E}+00$ & $0.0 \mathrm{E}+00$ \\
100 & $0.0 \mathrm{E}+00$ & $0.0 \mathrm{E}+00$ & $0.0 \mathrm{E}+00$ \\
125 & $0.0 \mathrm{E}+00$ & $0.0 \mathrm{E}+00$ & $0.0 \mathrm{E}+00$ \\
575 & $0.0 \mathrm{E}+00$ & $0.0 \mathrm{E}+00$ & $0.0 \mathrm{E}+00$ \\
590 & $0.0 \mathrm{E}+00$ & $0.0 \mathrm{E}+00$ & $0.0 \mathrm{E}+00$ \\
620 & $0.0 \mathrm{E}+00$ & $0.0 \mathrm{E}+00$ & $0.0 \mathrm{E}+00$ \\
750 & $0.0 \mathrm{E}+00$ & $0.0 \mathrm{E}+00$ & $0.0 \mathrm{E}+00$ \\
1000 & $0.0 \mathrm{E}+00$ & $0.0 \mathrm{E}+00$ & $0.0 \mathrm{E}+00$ \\
1015 & $0.0 \mathrm{E}+00$ & $0.0 \mathrm{E}+00$ & $0.0 \mathrm{E}+00$ \\
1025 & $0.0 \mathrm{E}+00$ & $0.0 \mathrm{E}+00$ & $0.0 \mathrm{E}+00$ \\
1060 & $0.0 \mathrm{E}+00$ & $0.0 \mathrm{E}+00$ & $0.0 \mathrm{E}+00$ \\
1080 & $0.0 \mathrm{E}+00$ & $0.0 \mathrm{E}+00$ & $0.0 \mathrm{E}+00$ \\
1120 & $0.0 \mathrm{E}+00$ & $0.0 \mathrm{E}+00$ & $0.0 \mathrm{E}+00$ \\
1175 & $0.0 \mathrm{E}+00$ & $0.0 \mathrm{E}+00$ & $0.0 \mathrm{E}+00$ \\
1575 & $0.0 \mathrm{E}+00$ & $0.0 \mathrm{E}+00$ & $0.0 \mathrm{E}+00$ \\
1750 & $0.0 \mathrm{E}+00$ & $0.0 \mathrm{E}+00$ & $0.0 \mathrm{E}+00$ \\
2175 & $0.0 \mathrm{E}+00$ & $0.0 \mathrm{E}+00$ & $0.0 \mathrm{E}+00$ \\
4000 & $0.0 \mathrm{E}+00$ & $0.0 \mathrm{E}+00$ & $0.0 \mathrm{E}+00$ \\
6000 & $7.3 \mathrm{E}-02$ & $0.0 \mathrm{E}+00$ & $0.0 \mathrm{E}+00$ \\
8000 & $4.5 \mathrm{E}-01$ & $0.0 \mathrm{E}+00$ & $0.0 \mathrm{E}+00$ \\
10000 & $4.5 \mathrm{E}-01$ & $0.0 \mathrm{E}+00$ & $0.0 \mathrm{E}+00$ \\
& & & \\
\hline & & &
\end{tabular}

GBBRM Cancer Risk from Beef Ingestion in Zone $B$ Due to Groundwater Release

\begin{tabular}{rrrr}
\hline Time $(y r)$ & As & $C r$ & $N i$ \\
\hline 15 & $0.0 \mathrm{E}+00$ & $0.0 \mathrm{E}+00$ & $0.0 \mathrm{E}+00$ \\
20 & $0.0 \mathrm{E}+00$ & $0.0 \mathrm{E}+00$ & $0.0 \mathrm{E}+00$ \\
25 & $0.0 \mathrm{E}+00$ & $0.0 \mathrm{E}+00$ & $0.0 \mathrm{E}+00$ \\
30 & $0.0 \mathrm{E}+00$ & $0.0 \mathrm{E}+00$ & $0.0 \mathrm{E}+00$ \\
60 & $0.0 \mathrm{E}+00$ & $0.0 \mathrm{E}+00$ & $0.0 \mathrm{E}+00$ \\
62 & $0.0 \mathrm{E}+00$ & $0.0 \mathrm{E}+00$ & $0.0 \mathrm{E}+00$ \\
80 & $0.0 \mathrm{E}+00$ & $0.0 \mathrm{E}+00$ & $0.0 \mathrm{E}+00$ \\
100 & $0.0 \mathrm{E}+00$ & $0.0 \mathrm{E}+00$ & $0.0 \mathrm{E}+00$ \\
125 & $0.0 \mathrm{E}+00$ & $0.0 \mathrm{E}+00$ & $0.0 \mathrm{E}+00$ \\
575 & $0.0 \mathrm{E}+00$ & $0.0 \mathrm{E}+00$ & $0.0 \mathrm{E}+00$ \\
590 & $0.0 \mathrm{E}+00$ & $0.0 \mathrm{E}+00$ & $0.0 \mathrm{E}+00$ \\
620 & $0.0 \mathrm{E}+00$ & $0.0 \mathrm{E}+00$ & $0.0 \mathrm{E}+00$ \\
750 & $0.0 \mathrm{E}+00$ & $0.0 \mathrm{E}+00$ & $0.0 \mathrm{E}+00$ \\
1000 & $0.0 \mathrm{E}+00$ & $0.0 \mathrm{E}+00$ & $0.0 \mathrm{E}+00$ \\
1015 & $0.0 \mathrm{E}+00$ & $0.0 \mathrm{E}+00$ & $0.0 \mathrm{E}+00$ \\
1025 & $0.0 \mathrm{E}+00$ & $0.0 \mathrm{E}+00$ & $0.0 \mathrm{E}+00$ \\
1060 & $0.0 \mathrm{E}+00$ & $0.0 \mathrm{E}+00$ & $0.0 \mathrm{E}+00$ \\
1080 & $0.0 \mathrm{E}+00$ & $0.0 \mathrm{E}+00$ & $0.0 \mathrm{E}+00$ \\
1120 & $0.0 \mathrm{E}+00$ & $0.0 \mathrm{E}+00$ & $0.0 \mathrm{E}+00$ \\
1175 & $0.0 \mathrm{E}+00$ & $0.0 \mathrm{E}+00$ & $0.0 \mathrm{E}+00$ \\
1575 & $0.0 \mathrm{E}+00$ & $0.0 \mathrm{E}+00$ & $0.0 \mathrm{E}+00$ \\
1750 & $0.0 \mathrm{E}+00$ & $0.0 \mathrm{E}+00$ & $0.0 \mathrm{E}+00$ \\
2175 & $0.0 \mathrm{E}+00$ & $0.0 \mathrm{E}+00$ & $0.0 \mathrm{E}+00$ \\
4000 & $0.0 \mathrm{E}+00$ & $0.0 \mathrm{E}+00$ & $0.0 \mathrm{E}+00$ \\
6000 & $8.7 \mathrm{E}-03$ & $0.0 \mathrm{E}+00$ & $0.0 \mathrm{E}+00$ \\
8000 & $5.4 \mathrm{E}-02$ & $0.0 \mathrm{E}+00$ & $0.0 \mathrm{E}+00$ \\
10000 & $5.4 \mathrm{E}-02$ & $0.0 \mathrm{E}+00$ & $0.0 \mathrm{E}+00$ \\
& & & \\
& & & \\
& &
\end{tabular}


GAFCU Concentration in Fish $\left(\mathrm{Bq} \mathrm{kg}^{-1}\right)$ in Zone $\mathrm{A}$ Due to Groundwater Release

\begin{tabular}{rlllll}
\hline Time $(Y r)$ & $\mathrm{U}-238 \& 4$ & $\mathrm{Th}-230$ & $\mathrm{Ra}-226$ & $\mathrm{~Pb}-210$ & $\mathrm{PO}-210$ \\
\hline 15 & $0.0 \mathrm{E}+00$ & $0.0 \mathrm{E}+00$ & $0.0 \mathrm{E}+00$ & $0.0 \mathrm{E}+00$ & $0.0 \mathrm{E}+00$ \\
20 & $0.0 \mathrm{E}+00$ & $0.0 \mathrm{E}+00$ & $0.0 \mathrm{E}+00$ & $0.0 \mathrm{E}+00$ & $0.0 \mathrm{E}+00$ \\
25 & $0.0 \mathrm{E}+00$ & $0.0 \mathrm{E}+00$ & $0.0 \mathrm{E}+00$ & $0.0 \mathrm{E}+00$ & $0.0 \mathrm{E}+00$ \\
30 & $0.0 \mathrm{E}+00$ & $0.0 \mathrm{E}+00$ & $0.0 \mathrm{E}+00$ & $0.0 \mathrm{E}+00$ & $0.0 \mathrm{E}+00$ \\
60 & $0.0 \mathrm{E}+00$ & $0.0 \mathrm{E}+00$ & $0.0 \mathrm{E}+00$ & $0.0 \mathrm{E}+00$ & $0.0 \mathrm{E}+00$ \\
62 & $1.1 \mathrm{E}+00$ & $4.3 \mathrm{E}-08$ & $5.0 \mathrm{E}-09$ & $2.8 \mathrm{E}-08$ & $4.6 \mathrm{E}-09$ \\
80 & $5.9 \mathrm{E}+01$ & $1.3 \mathrm{E}-04$ & $3.9 \mathrm{E}-06$ & $2.2 \mathrm{E}-05$ & $3.7 \mathrm{E}-06$ \\
100 & $1.2 \mathrm{E}+02$ & $5.7 \mathrm{E}-04$ & $3.5 \mathrm{E}-05$ & $2.0 \mathrm{E}-04$ & $3.3 \mathrm{E}-05$ \\
125 & $2.0 \mathrm{E}+02$ & $1.6 \mathrm{E}-03$ & $1.6 \mathrm{E}-04$ & $8.9 \mathrm{E}-04$ & $1.5 \mathrm{E}-04$ \\
575 & $2.0 \mathrm{E}+02$ & $2.3 \mathrm{E}-02$ & $2.5 \mathrm{E}-02$ & $1.4 \mathrm{E}-01$ & $2.3 \mathrm{E}-02$ \\
590 & $2.0 \mathrm{E}+02$ & $2.4 \mathrm{E}-02$ & $4.4 \mathrm{E}+00$ & $2.7 \mathrm{E}+01$ & $4.5 \mathrm{E}+00$ \\
620 & $2.0 \mathrm{E}+02$ & $2.5 \mathrm{E}-02$ & $4.4 \mathrm{E}+01$ & $2.7 \mathrm{E}+02$ & $4.4 \mathrm{E}+01$ \\
750 & $2.0 \mathrm{E}+02$ & $3.1 \mathrm{E}-02$ & $2.1 \mathrm{E}+02$ & $1.3 \mathrm{E}+03$ & $2.1 \mathrm{E}+02$ \\
1000 & $2.0 \mathrm{E}+02$ & $4.4 \mathrm{E}-02$ & $5.0 \mathrm{E}+02$ & $3.1 \mathrm{E}+03$ & $5.1 \mathrm{E}+02$ \\
1015 & $2.0 \mathrm{E}+02$ & $4.4 \mathrm{E}-02$ & $5.2 \mathrm{E}+02$ & $3.2 \mathrm{E}+03$ & $5.3 \mathrm{E}+02$ \\
1025 & $2.0 \mathrm{E}+02$ & $4.5 \mathrm{E}-02$ & $5.3 \mathrm{E}+02$ & $3.2 \mathrm{E}+03$ & $5.4 \mathrm{E}+02$ \\
1060 & $2.0 \mathrm{E}+02$ & $4.7 \mathrm{E}-02$ & $5.7 \mathrm{E}+02$ & $3.5 \mathrm{E}+03$ & $5.8 \mathrm{E}+02$ \\
1080 & $2.0 \mathrm{E}+02$ & $4.7 \mathrm{E}-02$ & $5.9 \mathrm{E}+02$ & $3.6 \mathrm{E}+03$ & $6.0 \mathrm{E}+02$ \\
1120 & $1.1 \mathrm{E}+01$ & $4.8 \mathrm{E}-02$ & $6.3 \mathrm{E}+02$ & $3.8 \mathrm{E}+03$ & $6.4 \mathrm{E}+02$ \\
1175 & $0.0 \mathrm{E}+00$ & $4.8 \mathrm{E}-02$ & $6.9 \mathrm{E}+02$ & $4.2 \mathrm{E}+03$ & $7.0 \mathrm{E}+02$ \\
1575 & $0.0 \mathrm{E}+00$ & $4.8 \mathrm{E}-02$ & $6.9 \mathrm{E}+02$ & $4.2 \mathrm{E}+03$ & $7.0 \mathrm{E}+02$ \\
1750 & $0.0 \mathrm{E}+00$ & $4.7 \mathrm{E}-02$ & $4.9 \mathrm{E}+02$ & $3.0 \mathrm{E}+03$ & $5.0 \mathrm{E}+02$ \\
2175 & $0.0 \mathrm{E}+00$ & $4.6 \mathrm{E}-02$ & $1.6 \mathrm{E}+01$ & $9.0 \mathrm{E}+01$ & $1.6 \mathrm{E}+01$ \\
4000 & $0.0 \mathrm{E}+00$ & $4.5 \mathrm{E}-02$ & $1.6 \mathrm{E}+01$ & $1.0 \mathrm{E}+02$ & $1.7 \mathrm{E}+01$ \\
6000 & $0.0 \mathrm{E}+00$ & $4.4 \mathrm{E}-02$ & $1.7 \mathrm{E}+01$ & $1.1 \mathrm{E}+02$ & $1.9 \mathrm{E}+01$ \\
8000 & $0.0 \mathrm{E}+00$ & $4.4 \mathrm{E}-02$ & $1.7 \mathrm{E}+01$ & $1.1 \mathrm{E}+02$ & $1.8 \mathrm{E}+01$ \\
10000 & $0.0 \mathrm{E}+00$ & $4.3 \mathrm{E}-02$ & $1.8 \mathrm{E}+01$ & $1.1 \mathrm{E}+02$ & $1.9 \mathrm{E}+01$ \\
& & & & & \\
\hline
\end{tabular}




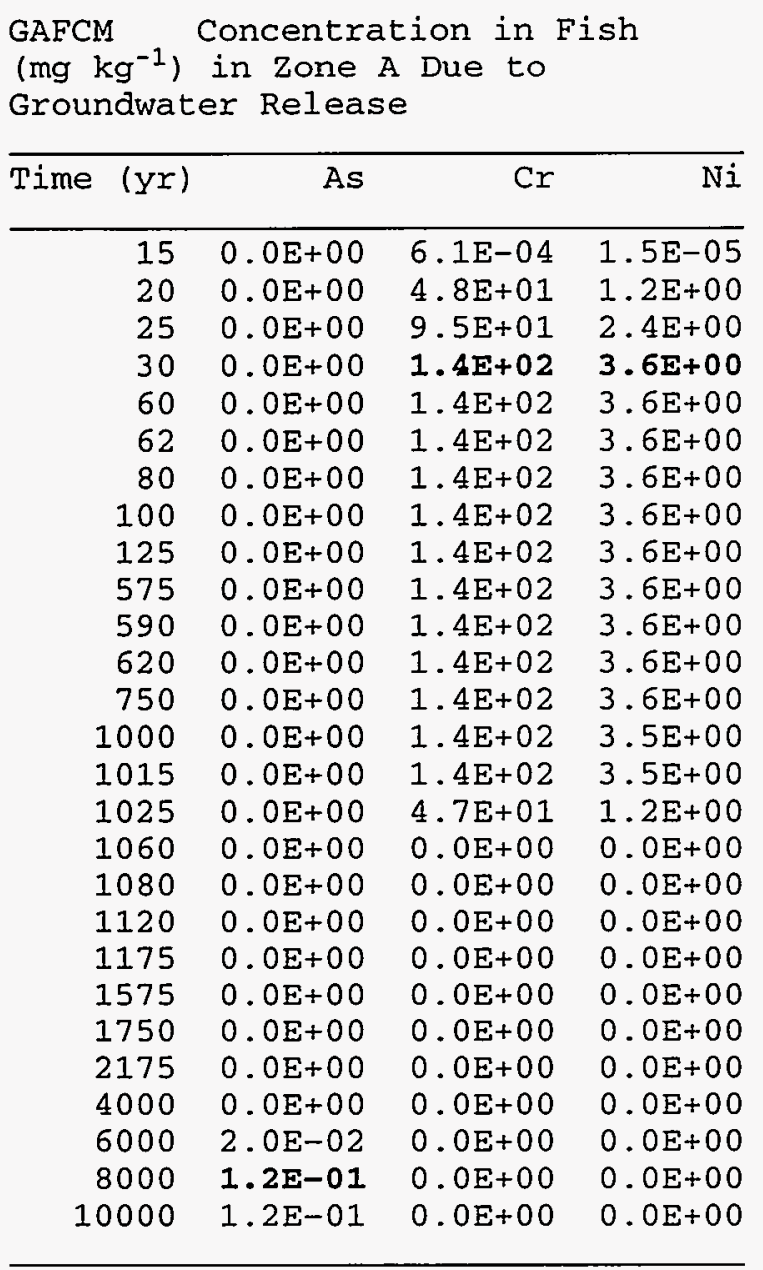


GAFDU Dose from Fish Ingestion in zone A Due to Groundwater Release ( $\mathrm{SV} \mathrm{yr}^{-1}$ )

\begin{tabular}{rcccccc}
\hline Time (Yr) & $\mathrm{U}-238 \& 4$ & Th-230 & Ra-226 & Pb-210 & Po-210 & Chain \\
\hline 15 & $0.0 \mathrm{E}+00$ & $0.0 \mathrm{E}+00$ & $0.0 \mathrm{E}+00$ & $0.0 \mathrm{E}+00$ & $0.0 \mathrm{E}+00$ & $0.0 \mathrm{E}+00$ \\
20 & $0.0 \mathrm{E}+00$ & $0.0 \mathrm{E}+00$ & $0.0 \mathrm{E}+00$ & $0.0 \mathrm{E}+00$ & $0.0 \mathrm{E}+00$ & $0.0 \mathrm{E}+00$ \\
25 & $0.0 \mathrm{E}+00$ & $0.0 \mathrm{E}+00$ & $0.0 \mathrm{E}+00$ & $0.0 \mathrm{E}+00$ & $0.0 \mathrm{E}+00$ & $0.0 \mathrm{E}+00$ \\
30 & $0.0 \mathrm{E}+00$ & $0.0 \mathrm{E}+00$ & $0.0 \mathrm{E}+00$ & $0.0 \mathrm{E}+00$ & $0.0 \mathrm{E}+00$ & $0.0 \mathrm{E}+00$ \\
60 & $0.0 \mathrm{E}+00$ & $0.0 \mathrm{E}+00$ & $0.0 \mathrm{E}+00$ & $0.0 \mathrm{E}+00$ & $0.0 \mathrm{E}+00$ & $0.0 \mathrm{E}+00$ \\
62 & $2.2 \mathrm{E}-07$ & $7.5 \mathrm{E}-14$ & $5.5 \mathrm{E}-15$ & $1.8 \mathrm{E}-13$ & $1.4 \mathrm{E}-14$ & $2.2 \mathrm{E}-07$ \\
80 & $1.2 \mathrm{E}-05$ & $2.3 \mathrm{E}-10$ & $4.3 \mathrm{E}-12$ & $1.4 \mathrm{E}-10$ & $1.1 \mathrm{E}-11$ & $1.2 \mathrm{E}-05$ \\
100 & $2.5 \mathrm{E}-05$ & $1.0 \mathrm{E}-09$ & $3.8 \mathrm{E}-11$ & $1.3 \mathrm{E}-09$ & $1.0 \mathrm{E}-10$ & $2.5 \mathrm{E}-05$ \\
125 & $4.1 \mathrm{E}-05$ & $2.7 \mathrm{E}-09$ & $1.7 \mathrm{E}-10$ & $5.8 \mathrm{E}-09$ & $4.6 \mathrm{E}-10$ & $4.1 \mathrm{E}-05$ \\
575 & $4.1 \mathrm{E}-05$ & $4.0 \mathrm{E}-08$ & $2.7 \mathrm{E}-08$ & $9.1 \mathrm{E}-07$ & $7.2 \mathrm{E}-08$ & $4.2 \mathrm{E}-05$ \\
590 & $4.1 \mathrm{E}-05$ & $4.1 \mathrm{E}-08$ & $4.9 \mathrm{E}-06$ & $1.8 \mathrm{E}-04$ & $1.4 \mathrm{E}-05$ & $2.3 \mathrm{E}-04$ \\
620 & $4.1 \mathrm{E}-05$ & $4.4 \mathrm{E}-08$ & $4.8 \mathrm{E}-05$ & $1.7 \mathrm{E}-03$ & $1.4 \mathrm{E}-04$ & $2.0 \mathrm{E}-03$ \\
750 & $4.1 \mathrm{E}-05$ & $5.5 \mathrm{E}-08$ & $2.3 \mathrm{E}-04$ & $8.3 \mathrm{E}-03$ & $6.6 \mathrm{E}-04$ & $9.2 \mathrm{E}-03$ \\
1000 & $4.1 \mathrm{E}-05$ & $7.6 \mathrm{E}-08$ & $5.5 \mathrm{E}-04$ & $2.0 \mathrm{E}-02$ & $1.6 \mathrm{E}-03$ & $2.2 \mathrm{E}-02$ \\
1015 & $4.1 \mathrm{E}-05$ & $7.8 \mathrm{E}-08$ & $5.7 \mathrm{E}-04$ & $2.1 \mathrm{E}-02$ & $1.6 \mathrm{E}-03$ & $2.3 \mathrm{E}-02$ \\
1025 & $4.1 \mathrm{E}-05$ & $7.9 \mathrm{E}-08$ & $5.8 \mathrm{E}-04$ & $2.1 \mathrm{E}-02$ & $1.7 \mathrm{E}-03$ & $2.3 \mathrm{E}-02$ \\
1060 & $4.1 \mathrm{E}-05$ & $8.2 \mathrm{E}-08$ & $6.3 \mathrm{E}-04$ & $2.3 \mathrm{E}-02$ & $1.8 \mathrm{E}-03$ & $2.5 \mathrm{E}-02$ \\
1080 & $4.0 \mathrm{E}-05$ & $8.3 \mathrm{E}-08$ & $6.5 \mathrm{E}-04$ & $2.3 \mathrm{E}-02$ & $1.9 \mathrm{E}-03$ & $2.6 \mathrm{E}-02$ \\
1120 & $2.3 \mathrm{E}-06$ & $8.4 \mathrm{E}-08$ & $7.0 \mathrm{E}-04$ & $2.5 \mathrm{E}-02$ & $2.0 \mathrm{E}-03$ & $2.8 \mathrm{E}-02$ \\
1175 & $0.0 \mathrm{E}+00$ & $8.4 \mathrm{E}-08$ & $7.6 \mathrm{E}-04$ & $2.7 \mathrm{E}-02$ & $2.2 \mathrm{E}-03$ & $3.0 \mathrm{E}-02$ \\
1575 & $0.0 \mathrm{E}+00$ & $8.3 \mathrm{E}-08$ & $7.6 \mathrm{E}-04$ & $2.7 \mathrm{E}-02$ & $2.2 \mathrm{E}-03$ & $3.0 \mathrm{E}-02$ \\
1750 & $0.0 \mathrm{E}+00$ & $8.2 \mathrm{E}-08$ & $5.4 \mathrm{E}-04$ & $1.9 \mathrm{E}-02$ & $1.5 \mathrm{E}-03$ & $2.1 \mathrm{E}-02$ \\
2175 & $0.0 \mathrm{E}+00$ & $8.1 \mathrm{E}-08$ & $1.7 \mathrm{E}-05$ & $6.0 \mathrm{E}-04$ & $5.0 \mathrm{E}-05$ & $6.7 \mathrm{E}-04$ \\
4000 & $0.0 \mathrm{E}+00$ & $8.0 \mathrm{E}-08$ & $1.8 \mathrm{E}-05$ & $6.0 \mathrm{E}-04$ & $5.0 \mathrm{E}-05$ & $6.7 \mathrm{E}-04$ \\
6000 & $0.0 \mathrm{E}+00$ & $7.8 \mathrm{E}-08$ & $1.8 \mathrm{E}-05$ & $7.0 \mathrm{E}-04$ & $5.0 \mathrm{E}-05$ & $7.7 \mathrm{E}-04$ \\
8000 & $0.0 \mathrm{E}+00$ & $7.7 \mathrm{E}-08$ & $2.0 \mathrm{E}-05$ & $7.0 \mathrm{E}-04$ & $6.0 \mathrm{E}-05$ & $7.8 \mathrm{E}-04$ \\
10000 & $0.0 \mathrm{E}+00$ & $7.6 \mathrm{E}-08$ & $2.0 \mathrm{E}-05$ & $7.0 \mathrm{E}-04$ & $6.0 \mathrm{E}-05$ & $7.8 \mathrm{E}-04$ \\
& & & & & & \\
\hline
\end{tabular}


GAFIM Intake from Fish

Ingestion in Zone A Due to

Groundwater Release ( $\mathrm{mg} Y \mathrm{Y}^{-1}$ )

\begin{tabular}{rccc}
\hline Time $(\mathrm{Yr})$ & As & $\mathrm{Cr}$ & $\mathrm{Ni}$ \\
\hline 15 & $0.0 \mathrm{E}+00$ & $3.0 \mathrm{E}-03$ & $7.6 \mathrm{E}-05$ \\
20 & $0.0 \mathrm{E}+00$ & $2.4 \mathrm{E}+02$ & $5.9 \mathrm{E}+00$ \\
25 & $0.0 \mathrm{E}+00$ & $4.8 \mathrm{E}+02$ & $1.2 \mathrm{E}+01$ \\
30 & $0.0 \mathrm{E}+00$ & $7.1 \mathrm{E}+02$ & $1.8 \mathrm{E}+01$ \\
60 & $0.0 \mathrm{E}+00$ & $7.1 \mathrm{E}+02$ & $1.8 \mathrm{E}+01$ \\
62 & $0.0 \mathrm{E}+00$ & $7.1 \mathrm{E}+02$ & $1.8 \mathrm{E}+01$ \\
80 & $0.0 \mathrm{E}+00$ & $7.1 \mathrm{E}+02$ & $1.8 \mathrm{E}+01$ \\
100 & $0.0 \mathrm{E}+00$ & $7.1 \mathrm{E}+02$ & $1.8 \mathrm{E}+01$ \\
125 & $0.0 \mathrm{E}+00$ & $7.1 \mathrm{E}+02$ & $1.8 \mathrm{E}+01$ \\
575 & $0.0 \mathrm{E}+00$ & $7.1 \mathrm{E}+02$ & $1.8 \mathrm{E}+01$ \\
590 & $0.0 \mathrm{E}+00$ & $7.1 \mathrm{E}+02$ & $1.8 \mathrm{E}+01$ \\
620 & $0.0 \mathrm{E}+00$ & $7.1 \mathrm{E}+02$ & $1.8 \mathrm{E}+01$ \\
750 & $0.0 \mathrm{E}+00$ & $7.1 \mathrm{E}+02$ & $1.8 \mathrm{E}+01$ \\
1000 & $0.0 \mathrm{E}+00$ & $7.1 \mathrm{E}+02$ & $1.8 \mathrm{E}+01$ \\
1015 & $0.0 \mathrm{E}+00$ & $7.1 \mathrm{E}+02$ & $1.8 \mathrm{E}+01$ \\
1025 & $0.0 \mathrm{E}+00$ & $2.4 \mathrm{E}+02$ & $5.9 \mathrm{E}+00$ \\
1060 & $0.0 \mathrm{E}+00$ & $0.0 \mathrm{E}+00$ & $0.0 \mathrm{E}+00$ \\
1080 & $0.0 \mathrm{E}+00$ & $0.0 \mathrm{E}+00$ & $0.0 \mathrm{E}+00$ \\
1120 & $0.0 \mathrm{E}+00$ & $0.0 \mathrm{E}+00$ & $0.0 \mathrm{E}+00$ \\
1175 & $0.0 \mathrm{E}+00$ & $0.0 \mathrm{E}+00$ & $0.0 \mathrm{E}+00$ \\
1575 & $0.0 \mathrm{E}+00$ & $0.0 \mathrm{E}+00$ & $0.0 \mathrm{E}+00$ \\
1750 & $0.0 \mathrm{E}+00$ & $0.0 \mathrm{E}+00$ & $0.0 \mathrm{E}+00$ \\
2175 & $0.0 \mathrm{E}+00$ & $0.0 \mathrm{E}+00$ & $0.0 \mathrm{E}+00$ \\
4000 & $0.0 \mathrm{E}+00$ & $0.0 \mathrm{E}+00$ & $0.0 \mathrm{E}+00$ \\
6000 & $1.0 \mathrm{E}-01$ & $0.0 \mathrm{E}+00$ & $0.0 \mathrm{E}+00$ \\
8000 & $6.1 \mathrm{E}-01$ & $0.0 \mathrm{E}+00$ & $0.0 \mathrm{E}+00$ \\
10000 & $6.1 \mathrm{E}-01$ & $0.0 \mathrm{E}+00$ & $0.0 \mathrm{E}+00$ \\
& & & \\
\hline & & & \\
& & &
\end{tabular}


GAFRU Cancer Risk from Fish Ingestion in zone A Due to Groundwater Release

\begin{tabular}{|c|c|c|c|c|c|c|}
\hline Time & $\mathrm{U}-238 \& 4$ & $\mathrm{Th}-230$ & $\mathrm{Ra}-226$ & $\mathrm{~Pb}-210$ & Po-210 & Chain \\
\hline 15 & $0.0 E+00$ & $0.0 \mathrm{E}+00$ & $0.0 E+00$ & $0.0 \mathrm{E}+00$ & $0.0 \mathrm{E}+00$ & $0.0 \mathrm{E}+00$ \\
\hline 20 & $0.0 \mathrm{E}+00$ & $0.0 E+00$ & $0.0 \mathrm{E}+00$ & $0.0 \mathrm{E}+00$ & $0.0 \mathrm{E}+00$ & $0.0 E+00$ \\
\hline 25 & $0.0 E+00$ & $0.0 E+00$ & $0.0 \mathrm{E}+00$ & $0.0 E+00$ & $0.0 E+00$ & $0.0 \mathrm{E}+00$ \\
\hline 30 & $0.0 E+00$ & $0.0 E+00$ & $0.0 \mathrm{E}+00$ & $0.0 \mathrm{E}+00$ & $0.0 E+00$ & $0.0 E+00$ \\
\hline 60 & $0.0 E+00$ & $0.0 E+00$ & $0.0 E+00$ & $0.0 \mathrm{E}+00$ & $0.0 \mathrm{E}+00$ & $0.0 \mathrm{E}+00$ \\
\hline 62 & $2.3 E-07$ & $5.3 E-15$ & $5.6 \mathrm{E}-15$ & $1.4 \mathrm{E}-13$ & $6.7 E-15$ & $2.3 E-07$ \\
\hline 80 & 1. $2 \mathrm{E}-05$ & 1. $6 E-11$ & 4. $4 \mathrm{E}-12$ & $1.1 \mathrm{E}-10$ & 5. $3 E-12$ & $1.2 \mathrm{E}-05$ \\
\hline 100 & $2.6 E-05$ & $7.0 \mathrm{E}-11$ & $3.9 \Xi-11$ & $9.6 \mathrm{E}-10$ & 4. $7 E-11$ & $2.6 \mathrm{E}-05$ \\
\hline 125 & $4.2 E-05$ & 1. $9 \mathrm{E}-10$ & 1. $8 \mathrm{E}-10$ & $4.3 E-09$ & $2.1 \mathrm{E}-10$ & $4.2 \mathrm{E}-05$ \\
\hline 575 & 4. $2 \mathrm{E}-05$ & $2.8 E-09$ & $2.8 \mathrm{E}-08$ & $6.8 \mathrm{E}-07$ & $3.3 E-08$ & $4.2 \mathrm{E}-05$ \\
\hline 590 & 4. $2 E-05$ & $2.9 E-09$ & $5.0 E-06$ & 1. $3 E-04$ & $6.5 E-06$ & 1. $9 \mathrm{E}-04$ \\
\hline 620 & 4. $2 E-05$ & $3.1 E-09$ & $4.9 E-05$ & 1. $3 E-03$ & $6.4 E-05$ & $1.5 \mathrm{E}-03$ \\
\hline 750 & 4. $2 E-05$ & 3. $8 E-09$ & $2.3 E-04$ & $6.2 \mathrm{E}-03$ & 3. $0 \mathrm{E}-04$ & $6.8 E-03$ \\
\hline 1000 & $4.2 E-05$ & $5.3 E-09$ & $5.6 \mathrm{E}-04$ & 1. $5 \mathrm{E}-02$ & $7.3 E-04$ & 1. $6 \mathrm{E}-02$ \\
\hline 1015 & $4.2 \mathrm{E}-05$ & $5.4 \mathrm{E}-09$ & $5.8 E-04$ & 1. $5 \mathrm{E}-02$ & $7.6 \mathrm{E}-04$ & $1.7 \mathrm{E}-02$ \\
\hline 1025 & 4. $2 \mathrm{E}-05$ & $5.5 E-09$ & $5.9 E-04$ & 1. $6 \mathrm{E}-02$ & $7.7 \mathrm{E}-04$ & $1.7 \mathrm{E}-02$ \\
\hline 1060 & 4. $2 E-05$ & $5.7 \mathrm{E}-09$ & $6.4 \mathrm{E}-04$ & 1. $7 \mathrm{E}-02$ & $8.3 E-04$ & $1.8 E-02$ \\
\hline 1080 & $4.1 E-05$ & $5.8 E-09$ & $6.6 \mathrm{E}-04$ & 1. $8 \mathrm{E}-02$ & $8.6 E-04$ & $1.9 \mathrm{E}-02$ \\
\hline 1120 & $2.3 E-06$ & $5.9 E-09$ & $7.1 \mathrm{E}-04$ & 1. $9 E-02$ & $9.2 \mathrm{E}-04$ & $2.1 E-02$ \\
\hline 1175 & $0.0 \mathrm{E}+00$ & $5.9 E-09$ & $7.7 E-04$ & $2.1 E-02$ & 1. $0 E-03$ & $2.2 \mathrm{E}-02$ \\
\hline 1575 & $0.0 E+00$ & $5.8 \mathrm{E}-09$ & $7.8 \mathrm{E}-04$ & $2.1 \mathrm{E}-02$ & 1. $0 \mathrm{E}-03$ & $2.3 E-02$ \\
\hline 1750 & $0.0 E+00$ & $5.8 E-09$ & $5.5 E-04$ & 1. $5 \mathrm{E}-02$ & $7.1 E-04$ & 1. $6 \mathrm{E}-02$ \\
\hline 2175 & $0.0 E+00$ & $5.7 E-09$ & 1. $8 \mathrm{E}-05$ & $4.7 E-04$ & $2.3 E-05$ & $5.1 E-04$ \\
\hline 4000 & $0.0 E+00$ & $5.6 \mathrm{E}-09$ & 1. $8 E-05$ & $4.8 E-04$ & $2.4 \mathrm{E}-05$ & $5.2 \mathrm{E}-04$ \\
\hline 6000 & $0.0 \mathrm{E}+00$ & $5.5 E-09$ & $1.9 \mathrm{E}-05$ & $5.4 \mathrm{E}-04$ & $2.6 \mathrm{E}-05$ & $5.8 E-04$ \\
\hline 8000 & $0.0 E+00$ & $5.4 \mathrm{E}-09$ & $2.0 \mathrm{E}-05$ & 5. $2 E-04$ & $2.5 E-05$ & $5.6 \mathrm{E}-04$ \\
\hline 10000 & $0.0 E+00$ & $5.3 E-09$ & $2.1 \mathrm{E}-05$ & $5.5 E-04$ & $2.7 E-05$ & $6.0 \mathrm{E}-04$ \\
\hline
\end{tabular}


GAFRM Cancer Risk from Fish

Ingestion in Zone A Due to

Groundwater Release

\begin{tabular}{rccr}
\hline Time $(\mathrm{Yr})$ & As & $\mathrm{Cr}$ & $\mathrm{Ni}$ \\
\hline 15 & $0.0 \mathrm{E}+00$ & $0.0 \mathrm{E}+00$ & $0.0 \mathrm{E}+00$ \\
20 & $0.0 \mathrm{E}+00$ & $0.0 \mathrm{E}+00$ & $0.0 \mathrm{E}+00$ \\
25 & $0.0 \mathrm{E}+00$ & $0.0 \mathrm{E}+00$ & $0.0 \mathrm{E}+00$ \\
30 & $0.0 \mathrm{E}+00$ & $0.0 \mathrm{E}+00$ & $0.0 \mathrm{E}+00$ \\
60 & $0.0 \mathrm{E}+00$ & $0.0 \mathrm{E}+00$ & $0.0 \mathrm{E}+00$ \\
62 & $0.0 \mathrm{E}+00$ & $0.0 \mathrm{E}+00$ & $0.0 \mathrm{E}+00$ \\
80 & $0.0 \mathrm{E}+00$ & $0.0 \mathrm{E}+00$ & $0.0 \mathrm{E}+00$ \\
100 & $0.0 \mathrm{E}+00$ & $0.0 \mathrm{E}+00$ & $0.0 \mathrm{E}+00$ \\
125 & $0.0 \mathrm{E}+00$ & $0.0 \mathrm{E}+00$ & $0.0 \mathrm{E}+00$ \\
575 & $0.0 \mathrm{E}+00$ & $0.0 \mathrm{E}+00$ & $0.0 \mathrm{E}+00$ \\
590 & $0.0 \mathrm{E}+00$ & $0.0 \mathrm{E}+00$ & $0.0 \mathrm{E}+00$ \\
620 & $0.0 \mathrm{E}+00$ & $0.0 \mathrm{E}+00$ & $0.0 \mathrm{E}+00$ \\
750 & $0.0 \mathrm{E}+00$ & $0.0 \mathrm{E}+00$ & $0.0 \mathrm{E}+00$ \\
1000 & $0.0 \mathrm{E}+00$ & $0.0 \mathrm{E}+00$ & $0.0 \mathrm{E}+00$ \\
1015 & $0.0 \mathrm{E}+00$ & $0.0 \mathrm{E}+00$ & $0.0 \mathrm{E}+00$ \\
1025 & $0.0 \mathrm{E}+00$ & $0.0 \mathrm{E}+00$ & $0.0 \mathrm{E}+00$ \\
1060 & $0.0 \mathrm{E}+00$ & $0.0 \mathrm{E}+00$ & $0.0 \mathrm{E}+00$ \\
1080 & $0.0 \mathrm{E}+00$ & $0.0 \mathrm{E}+00$ & $0.0 \mathrm{E}+00$ \\
1120 & $0.0 \mathrm{E}+00$ & $0.0 \mathrm{E}+00$ & $0.0 \mathrm{E}+00$ \\
1175 & $0.0 \mathrm{E}+00$ & $0.0 \mathrm{E}+00$ & $0.0 \mathrm{E}+00$ \\
1575 & $0.0 \mathrm{E}+00$ & $0.0 \mathrm{E}+00$ & $0.0 \mathrm{E}+00$ \\
1750 & $0.0 \mathrm{E}+00$ & $0.0 \mathrm{E}+00$ & $0.0 \mathrm{E}+00$ \\
2175 & $0.0 \mathrm{E}+00$ & $0.0 \mathrm{E}+00$ & $0.0 \mathrm{E}+00$ \\
4000 & $0.0 \mathrm{E}+00$ & $0.0 \mathrm{E}+00$ & $0.0 \mathrm{E}+00$ \\
6000 & $1.7 \mathrm{E}-03$ & $0.0 \mathrm{E}+00$ & $0.0 \mathrm{E}+00$ \\
8000 & $1.1 \mathrm{E}-02$ & $0.0 \mathrm{E}+00$ & $0.0 \mathrm{E}+00$ \\
10000 & $1.1 \mathrm{E}-02$ & $0.0 \mathrm{E}+00$ & $0.0 \mathrm{E}+00$ \\
& & & \\
& & & \\
& & &
\end{tabular}


GBLCU Concentration in Lake water $\left(\mathrm{Bq} \mathrm{L} \mathrm{L}^{-1}\right)$ in zone $\mathrm{B}$ Due to Groundwater Release

\begin{tabular}{rccccc}
\hline Time (yr) & $\mathrm{U}-238 \& 4$ & Th-230 & Ra-226 & Pb-210 & PO-210 \\
\hline 15 & $0.0 \mathrm{E}+00$ & $0.0 \mathrm{E}+00$ & $0.0 \mathrm{E}+00$ & $0.0 \mathrm{E}+00$ & $0.0 \mathrm{E}+00$ \\
20 & $0.0 \mathrm{E}+00$ & $0.0 \mathrm{E}+00$ & $0.0 \mathrm{E}+00$ & $0.0 \mathrm{E}+00$ & $0.0 \mathrm{E}+00$ \\
25 & $0.0 \mathrm{E}+00$ & $0.0 \mathrm{E}+00$ & $0.0 \mathrm{E}+00$ & $0.0 \mathrm{E}+00$ & $0.0 \mathrm{E}+00$ \\
30 & $0.0 \mathrm{E}+00$ & $0.0 \mathrm{E}+00$ & $0.0 \mathrm{E}+00$ & $0.0 \mathrm{E}+00$ & $0.0 \mathrm{E}+00$ \\
60 & $0.0 \mathrm{E}+00$ & $0.0 \mathrm{E}+00$ & $0.0 \mathrm{E}+00$ & $0.0 \mathrm{E}+00$ & $0.0 \mathrm{E}+00$ \\
62 & $1.1 \mathrm{E}-01$ & $4.3 \mathrm{E}-10$ & $9.9 \mathrm{E}-11$ & $9.3 \mathrm{E}-11$ & $9.3 \mathrm{E}-11$ \\
80 & $5.9 \mathrm{E}+00$ & $1.3 \mathrm{E}-06$ & $7.9 \mathrm{E}-08$ & $7.4 \mathrm{E}-08$ & $7.4 \mathrm{E}-08$ \\
100 & $1.2 \mathrm{E}+01$ & $5.7 \mathrm{E}-06$ & $6.9 \mathrm{E}-07$ & $6.5 \mathrm{E}-07$ & $6.5 \mathrm{E}-07$ \\
125 & $2.0 \mathrm{E}+01$ & $1.6 \mathrm{E}-05$ & $3.2 \mathrm{E}-06$ & $3.0 \mathrm{E}-06$ & $3.0 \mathrm{E}-06$ \\
575 & $2.0 \mathrm{E}+01$ & $2.3 \mathrm{E}-04$ & $5.0 \mathrm{E}-04$ & $4.7 \mathrm{E}-04$ & $4.6 \mathrm{E}-04$ \\
590 & $2.0 \mathrm{E}+01$ & $2.4 \mathrm{E}-04$ & $8.9 \mathrm{E}-02$ & $9.0 \mathrm{E}-02$ & $9.0 \mathrm{E}-02$ \\
620 & $2.0 \mathrm{E}+01$ & $2.5 \mathrm{E}-04$ & $8.8 \mathrm{E}-01$ & $8.9 \mathrm{E}-01$ & $8.9 \mathrm{E}-01$ \\
750 & $2.0 \mathrm{E}+01$ & $3.1 \mathrm{E}-04$ & $4.2 \mathrm{E}+00$ & $4.2 \mathrm{E}+00$ & $4.2 \mathrm{E}+00$ \\
1000 & $2.0 \mathrm{E}+01$ & $4.4 \mathrm{E}-04$ & $1.0 \mathrm{E}+01$ & $1.0 \mathrm{E}+01$ & $1.0 \mathrm{E}+01$ \\
1015 & $2.0 \mathrm{E}+01$ & $4.4 \mathrm{E}-04$ & $1.0 \mathrm{E}+01$ & $1.1 \mathrm{E}+01$ & $1.1 \mathrm{E}+01$ \\
1025 & $2.0 \mathrm{E}+01$ & $4.5 \mathrm{E}-04$ & $1.1 \mathrm{E}+01$ & $1.1 \mathrm{E}+01$ & $1.1 \mathrm{E}+01$ \\
1060 & $2.0 \mathrm{E}+01$ & $4.7 \mathrm{E}-04$ & $1.1 \mathrm{E}+01$ & $1.2 \mathrm{E}+01$ & $1.2 \mathrm{E}+01$ \\
1080 & $2.0 \mathrm{E}+01$ & $4.7 \mathrm{E}-04$ & $1.2 \mathrm{E}+01$ & $1.2 \mathrm{E}+01$ & $1.2 \mathrm{E}+01$ \\
1120 & $1.1 \mathrm{E}+00$ & $4.8 \mathrm{E}-04$ & $1.3 \mathrm{E}+01$ & $1.3 \mathrm{E}+01$ & $1.3 \mathrm{E}+01$ \\
1175 & $0.0 \mathrm{E}+00$ & $4.8 \mathrm{E}-04$ & $1.4 \mathrm{E}+01$ & $1.4 \mathrm{E}+01$ & $1.4 \mathrm{E}+01$ \\
1575 & $0.0 \mathrm{E}+00$ & $4.8 \mathrm{E}-04$ & $1.4 \mathrm{E}+01$ & $1.4 \mathrm{E}+01$ & $1.4 \mathrm{E}+01$ \\
1750 & $0.0 \mathrm{E}+00$ & $4.7 \mathrm{E}-04$ & $9.8 \mathrm{E}+00$ & $1.0 \mathrm{E}+01$ & $1.0 \mathrm{E}+01$ \\
2175 & $0.0 \mathrm{E}+00$ & $4.6 \mathrm{E}-04$ & $3.0 \mathrm{E}-01$ & $3.0 \mathrm{E}-01$ & $3.0 \mathrm{E}-01$ \\
4000 & $0.0 \mathrm{E}+00$ & $4.5 \mathrm{E}-04$ & $3.0 \mathrm{E}-01$ & $3.0 \mathrm{E}-01$ & $3.0 \mathrm{E}-01$ \\
6000 & $0.0 \mathrm{E}+00$ & $4.4 \mathrm{E}-04$ & $4.0 \mathrm{E}-01$ & $3.0 \mathrm{E}-01$ & $3.0 \mathrm{E}-01$ \\
8000 & $0.0 \mathrm{E}+00$ & $4.4 \mathrm{E}-04$ & $3.0 \mathrm{E}-01$ & $4.0 \mathrm{E}-01$ & $4.0 \mathrm{E}-01$ \\
10000 & $0.0 \mathrm{E}+00$ & $4.3 \mathrm{E}-04$ & $4.0 \mathrm{E}-01$ & $4.0 \mathrm{E}-01$ & $4.0 \mathrm{E}-01$ \\
& & & & & \\
\hline
\end{tabular}




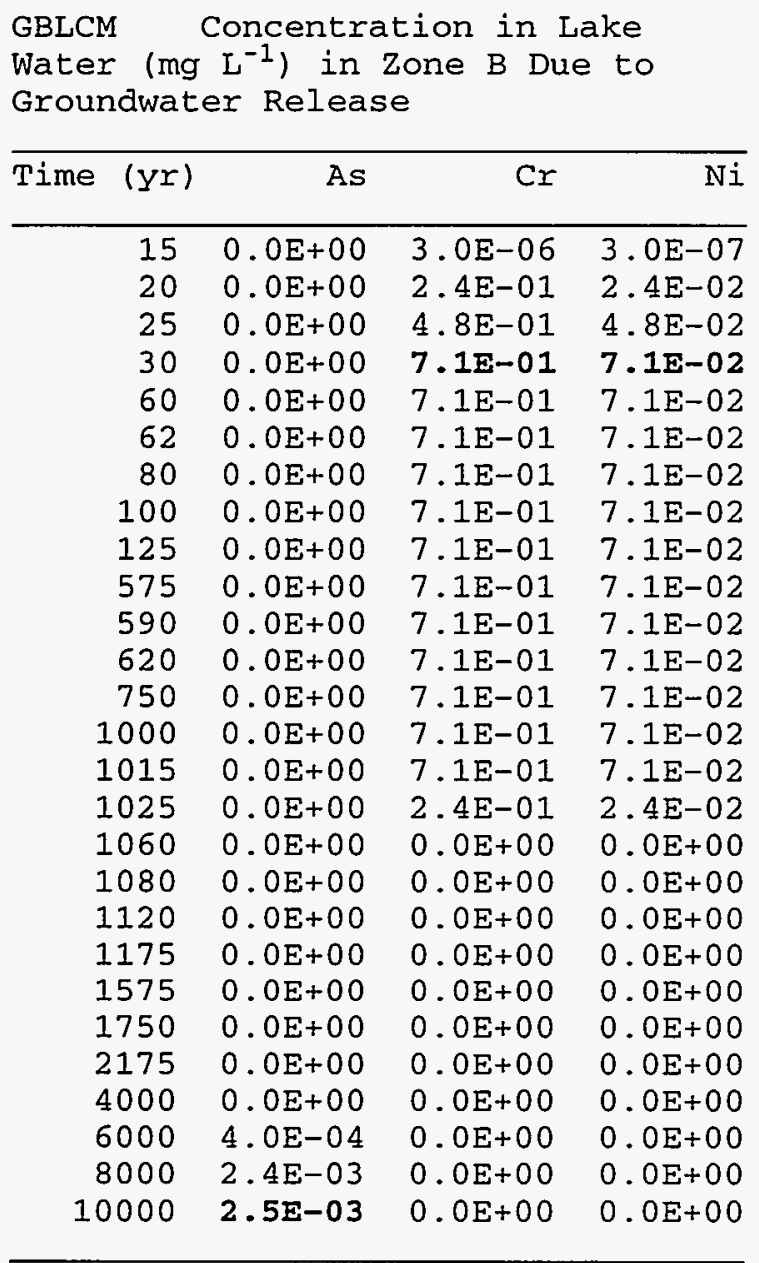


GBLDU Dose from Drinking water in Zone B Due to Groundwater Release (Sv $\mathrm{yr}^{-1}$ )

\begin{tabular}{|c|c|c|c|c|c|c|}
\hline Time (yr) & $\mathrm{U}-238 \& 4$ & Th -230 & $\mathrm{Ra}-226$ & $\mathrm{~Pb}-210$ & Po-210 & Chain \\
\hline 15 & $0.0 \mathrm{E}+00$ & $0.0 E+00$ & $0.0 \mathrm{E}+00$ & $0.0 \mathrm{E}+00$ & $0.0 \mathrm{E}+00$ & $0.0 \mathrm{E}+00$ \\
\hline 20 & $0.0 E+00$ & $0.0 \mathrm{E}+00$ & $0.0 \mathrm{E}+00$ & $0.0 E+00$ & $0.0 E+00$ & $0.0 E+00$ \\
\hline 25 & $0.0 E+00$ & $0.0 E+00$ & $0.0 E+00$ & $0.0 E+00$ & $0.0 E+00$ & $0.0 E+00$ \\
\hline 30 & $0.0 E+00$ & $0.0 E+00$ & $0.0 E+00$ & $0.0 \mathrm{E}+00$ & $0.0 E+00$ & $0.0 \mathrm{E}+00$ \\
\hline 60 & $0.0 E+00$ & $0.0 \mathrm{E}+00$ & $0.0 \mathrm{E}+00$ & $0.0 \mathrm{E}+00$ & $0.0 E+00$ & $0.0 \mathrm{E}+00$ \\
\hline 62 & $3.2 \mathrm{E}-06$ & 1. $1 E-13$ & 1. $6 \mathrm{E}-14$ & $8.8 \mathrm{E}-14$ & $4.2 \mathrm{E}-14$ & $3.2 \mathrm{E}-06$ \\
\hline 80 & $1.8 E-04$ & $3.3 E-10$ & 1. $3 E-11$ & $7.0 \mathrm{E}-11$ & 3. $3 E-11$ & $1.8 \mathrm{E}-04$ \\
\hline 100 & $3.7 E-04$ & 1. $5 E-09$ & 1. $1 \mathrm{E}-10$ & 6. $2 \mathrm{E}-10$ & $2.9 E-10$ & $3.7 E-04$ \\
\hline 125 & $5.9 E-04$ & 4. $0 E-09$ & $5.1 E-10$ & $2.8 E-09$ & $1.3 E-09$ & $5.9 \mathrm{E}-04$ \\
\hline 575 & $5.9 E-04$ & $5.9 E-08$ & $8.0 E-08$ & $4.4 \mathrm{E}-07$ & $2.1 \mathrm{E}-07$ & $5.9 \mathrm{E}-04$ \\
\hline 590 & $5.9 E-04$ & $6.0 E-08$ & 1. $4 \mathrm{E}-05$ & $8.5 E-05$ & $4.1 \mathrm{E}-05$ & $7.3 E-04$ \\
\hline 620 & $5.9 \mathrm{E}-04$ & $6.4 \mathrm{E}-08$ & 1. $4 \mathrm{E}-04$ & $8.4 E-04$ & $4.0 \mathrm{E}-04$ & $2.0 \mathrm{E}-03$ \\
\hline 750 & $5.9 E-04$ & $8.0 E-08$ & $6.7 E-04$ & $4.0 E-03$ & $1.9 E-03$ & $7.2 \mathrm{E}-03$ \\
\hline 1000 & $5.9 E-04$ & 1. $1 E-07$ & $1.6 \mathrm{E}-03$ & $9.7 \mathrm{E}-03$ & $4.6 E-03$ & $1.7 \mathrm{E}-02$ \\
\hline 1015 & $5.9 E-04$ & 1. $1 \mathrm{E}-07$ & 1. $7 E-03$ & 1. $0 \mathrm{E}-02$ & $4.8 E-03$ & $1.7 \mathrm{E}-02$ \\
\hline 1025 & $5.9 \mathrm{E}-04$ & $1.2 \mathrm{E}-07$ & $1.7 E-03$ & 1. $0 E-02$ & $4.9 \mathrm{E}-03$ & 1. $7 \mathrm{E}-02$ \\
\hline 1060 & $5.9 E-04$ & 1. $2 \mathrm{E}-07$ & $1.8 \mathrm{E}-03$ & $1.1 E-02$ & $5.2 E-03$ & 1. $9 E-02$ \\
\hline 1080 & $5.9 E-04$ & 1. $2 \mathrm{E}-07$ & 1. $9 E-03$ & 1. $1 E-02$ & $5.4 E-03$ & $1.9 \mathrm{E}-02$ \\
\hline 1120 & $3.1 E-05$ & $1.2 E-07$ & 2. $.0 E-03$ & 1. $2 \mathrm{E}-02$ & $5.8 \mathrm{E}-03$ & $2.0 \mathrm{E}-02$ \\
\hline 1175 & $0.0 E+00$ & 1. $2 \mathrm{E}-07$ & $2.2 E-03$ & 1. $3 E-02$ & $6.3 E-03$ & $2.2 \mathrm{E}-02$ \\
\hline 1575 & $0.0 E+00$ & $1.2 \mathrm{E}-07$ & $2.2 E-03$ & $1.3 \mathrm{E}-02$ & $6.4 E-03$ & $2.2 \mathrm{E}-02$ \\
\hline 1750 & $0.0 \mathrm{E}+00$ & $1.2 \mathrm{E}-07$ & 1. $6 \mathrm{E}-03$ & $9.4 \mathrm{E}-03$ & $4.5 E-03$ & 1. $5 E-02$ \\
\hline 2175 & $0.0 \mathrm{E}+00$ & 1. $2 \mathrm{E}-07$ & $5.0 E-05$ & $3.0 E-04$ & 1. $4 E-04$ & $4.9 \mathrm{E}-04$ \\
\hline 4000 & $0.0 E+00$ & 1. $2 \mathrm{E}-07$ & $5.0 \mathrm{E}-05$ & $3.0 E-04$ & 1. $4 \mathrm{E}-04$ & $4.9 \mathrm{E}-04$ \\
\hline 6000 & $0.0 E+00$ & 1.1E-07 & $6.0 \mathrm{E}-05$ & $4.0 E-04$ & 1. $7 E-04$ & $6.3 E-04$ \\
\hline 8000 & $0.0 \mathrm{E}+00$ & $1.1 E-07$ & $6.0 \mathrm{E}-05$ & 3. $0 \mathrm{E}-04$ & 1. $6 \mathrm{E}-04$ & $5.2 E-04$ \\
\hline 10000 & $0.0 \mathrm{E}+00$ & $1.1 \mathrm{E}-07$ & $6.0 E-05$ & $4.0 \mathrm{E}-04$ & 1. $6 \mathrm{E}-04$ & $6.2 \mathrm{E}-04$ \\
\hline
\end{tabular}


GBIIM Intake from Drinking

water in Zone B Due to Groundwater Release ( $m g \mathrm{yr}^{-1}$ )

\begin{tabular}{rrrr}
\hline Time (Yr) & As & $\mathrm{Cr}$ & $\mathrm{Ni}$ \\
\hline 15 & $0.0 \mathrm{E}+00$ & $2.2 \mathrm{E}-03$ & $2.2 \mathrm{E}-04$ \\
20 & $0.0 \mathrm{E}+00$ & $1.7 \mathrm{E}+02$ & $1.7 \mathrm{E}+01$ \\
25 & $0.0 \mathrm{E}+00$ & $3.5 \mathrm{E}+02$ & $3.5 \mathrm{E}+01$ \\
30 & $0.0 \mathrm{E}+00$ & $5.2 \mathrm{E}+02$ & $5.2 \mathrm{E}+01$ \\
60 & $0.0 \mathrm{E}+00$ & $5.2 \mathrm{E}+02$ & $5.2 \mathrm{E}+01$ \\
62 & $0.0 \mathrm{E}+00$ & $5.2 \mathrm{E}+02$ & $5.2 \mathrm{E}+01$ \\
80 & $0.0 \mathrm{E}+00$ & $5.2 \mathrm{E}+02$ & $5.2 \mathrm{E}+01$ \\
100 & $0.0 \mathrm{E}+00$ & $5.2 \mathrm{E}+02$ & $5.2 \mathrm{E}+01$ \\
125 & $0.0 \mathrm{E}+00$ & $5.2 \mathrm{E}+02$ & $5.2 \mathrm{E}+01$ \\
575 & $0.0 \mathrm{E}+00$ & $5.2 \mathrm{E}+02$ & $5.2 \mathrm{E}+01$ \\
590 & $0.0 \mathrm{E}+00$ & $5.2 \mathrm{E}+02$ & $5.2 \mathrm{E}+01$ \\
620 & $0.0 \mathrm{E}+00$ & $5.2 \mathrm{E}+02$ & $5.2 \mathrm{E}+01$ \\
750 & $0.0 \mathrm{E}+00$ & $5.2 \mathrm{E}+02$ & $5.2 \mathrm{E}+01$ \\
1000 & $0.0 \mathrm{E}+00$ & $5.2 \mathrm{E}+02$ & $5.2 \mathrm{E}+01$ \\
1015 & $0.0 \mathrm{E}+00$ & $5.2 \mathrm{E}+02$ & $5.2 \mathrm{E}+01$ \\
1025 & $0.0 \mathrm{E}+00$ & $1.7 \mathrm{E}+02$ & $1.7 \mathrm{E}+01$ \\
1060 & $0.0 \mathrm{E}+00$ & $0.0 \mathrm{E}+00$ & $0.0 \mathrm{E}+00$ \\
1080 & $0.0 \mathrm{E}+00$ & $0.0 \mathrm{E}+00$ & $0.0 \mathrm{E}+00$ \\
1120 & $0.0 \mathrm{E}+00$ & $0.0 \mathrm{E}+00$ & $0.0 \mathrm{E}+00$ \\
1175 & $0.0 \mathrm{E}+00$ & $0.0 \mathrm{E}+00$ & $0.0 \mathrm{E}+00$ \\
1575 & $0.0 \mathrm{E}+00$ & $0.0 \mathrm{E}+00$ & $0.0 \mathrm{E}+00$ \\
1750 & $0.0 \mathrm{E}+00$ & $0.0 \mathrm{E}+00$ & $0.0 \mathrm{E}+00$ \\
2175 & $0.0 \mathrm{E}+00$ & $0.0 \mathrm{E}+00$ & $0.0 \mathrm{E}+00$ \\
4000 & $0.0 \mathrm{E}+00$ & $0.0 \mathrm{E}+00$ & $0.0 \mathrm{E}+00$ \\
6000 & $2.9 \mathrm{E}-01$ & $0.0 \mathrm{E}+00$ & $0.0 \mathrm{E}+00$ \\
8000 & $1.8 \mathrm{E}+00$ & $0.0 \mathrm{E}+00$ & $0.0 \mathrm{E}+00$ \\
10000 & $1.8 \mathrm{E}+00$ & $0.0 \mathrm{E}+00$ & $0.0 \mathrm{E}+00$ \\
& & & \\
\hline & & &
\end{tabular}


GBLRU Cancer risk from Drinking water in zone B Due to Groundwater Release

\begin{tabular}{rllllll}
\hline Time (yr) & $\mathrm{U}-238 \& 4$ & $\mathrm{Th}-230$ & $\mathrm{Ra}-226$ & $\mathrm{~Pb}-210$ & PO-210 & Chain \\
\hline 15 & $0.0 \mathrm{E}+00$ & $0.0 \mathrm{E}+00$ & $0.0 \mathrm{E}+00$ & $0.0 \mathrm{E}+00$ & $0.0 \mathrm{E}+00$ & $0.0 \mathrm{E}+00$ \\
20 & $0.0 \mathrm{E}+00$ & $0.0 \mathrm{E}+00$ & $0.0 \mathrm{E}+00$ & $0.0 \mathrm{E}+00$ & $0.0 \mathrm{E}+00$ & $0.0 \mathrm{E}+00$ \\
25 & $0.0 \mathrm{E}+00$ & $0.0 \mathrm{E}+00$ & $0.0 \mathrm{E}+00$ & $0.0 \mathrm{E}+00$ & $0.0 \mathrm{E}+00$ & $0.0 \mathrm{E}+00$ \\
30 & $0.0 \mathrm{E}+00$ & $0.0 \mathrm{E}+00$ & $0.0 \mathrm{E}+00$ & $0.0 \mathrm{E}+00$ & $0.0 \mathrm{E}+00$ & $0.0 \mathrm{E}+00$ \\
60 & $0.0 \mathrm{E}+00$ & $0.0 \mathrm{E}+00$ & $0.0 \mathrm{E}+00$ & $0.0 \mathrm{E}+00$ & $0.0 \mathrm{E}+00$ & $0.0 \mathrm{E}+00$ \\
62 & $3.3 \mathrm{E}-06$ & $7.7 \mathrm{E}-15$ & $1.6 \mathrm{E}-14$ & $6.6 \mathrm{E}-14$ & $1.9 \mathrm{E}-14$ & $3.3 \mathrm{E}-06$ \\
80 & $1.8 \mathrm{E}-04$ & $2.3 \mathrm{E}-11$ & $1.3 \mathrm{E}-11$ & $5.3 \mathrm{E}-11$ & $1.5 \mathrm{E}-11$ & $1.8 \mathrm{E}-04$ \\
100 & $3.8 \mathrm{E}-04$ & $1.0 \mathrm{E}-10$ & $1.1 \mathrm{E}-10$ & $4.7 \mathrm{E}-10$ & $1.4 \mathrm{E}-10$ & $3.8 \mathrm{E}-04$ \\
125 & $6.1 \mathrm{E}-04$ & $2.8 \mathrm{E}-10$ & $5.1 \mathrm{E}-10$ & $2.1 \mathrm{E}-09$ & $6.2 \mathrm{E}-10$ & $6.1 \mathrm{E}-04$ \\
575 & $6.1 \mathrm{E}-04$ & $4.1 \mathrm{E}-09$ & $8.1 \mathrm{E}-08$ & $3.3 \mathrm{E}-07$ & $9.7 \mathrm{E}-08$ & $6.1 \mathrm{E}-04$ \\
590 & $6.1 \mathrm{E}-04$ & $4.2 \mathrm{E}-09$ & $1.5 \mathrm{E}-05$ & $6.4 \mathrm{E}-05$ & $1.9 \mathrm{E}-05$ & $7.1 \mathrm{E}-04$ \\
620 & $6.1 \mathrm{E}-04$ & $4.5 \mathrm{E}-09$ & $1.4 \mathrm{E}-04$ & $6.4 \mathrm{E}-04$ & $1.9 \mathrm{E}-04$ & $1.6 \mathrm{E}-03$ \\
750 & $6.1 \mathrm{E}-04$ & $5.6 \mathrm{E}-09$ & $6.8 \mathrm{E}-04$ & $3.0 \mathrm{E}-03$ & $8.9 \mathrm{E}-04$ & $5.2 \mathrm{E}-03$ \\
1000 & $6.1 \mathrm{E}-04$ & $7.8 \mathrm{E}-09$ & $1.6 \mathrm{E}-03$ & $7.3 \mathrm{E}-03$ & $2.1 \mathrm{E}-03$ & $1.2 \mathrm{E}-02$ \\
1015 & $6.1 \mathrm{E}-04$ & $7.9 \mathrm{E}-09$ & $1.7 \mathrm{E}-03$ & $7.5 \mathrm{E}-03$ & $2.2 \mathrm{E}-03$ & $1.2 \mathrm{E}-02$ \\
1025 & $6.1 \mathrm{E}-04$ & $8.0 \mathrm{E}-09$ & $1.7 \mathrm{E}-03$ & $7.7 \mathrm{E}-03$ & $2.3 \mathrm{E}-03$ & $1.2 \mathrm{E}-02$ \\
1060 & $6.1 \mathrm{E}-04$ & $8.3 \mathrm{E}-09$ & $1.9 \mathrm{E}-03$ & $8.3 \mathrm{E}-03$ & $2.4 \mathrm{E}-03$ & $1.3 \mathrm{E}-02$ \\
1080 & $6.0 \mathrm{E}-04$ & $8.5 \mathrm{E}-09$ & $1.9 \mathrm{E}-03$ & $8.6 \mathrm{E}-03$ & $2.5 \mathrm{E}-03$ & $1.4 \mathrm{E}-02$ \\
1120 & $3.3 \mathrm{E}-05$ & $8.6 \mathrm{E}-09$ & $2.1 \mathrm{E}-03$ & $9.2 \mathrm{E}-03$ & $2.7 \mathrm{E}-03$ & $1.4 \mathrm{E}-02$ \\
1175 & $0.0 \mathrm{E}+00$ & $8.6 \mathrm{E}-09$ & $2.3 \mathrm{E}-03$ & $1.0 \mathrm{E}-02$ & $2.9 \mathrm{E}-03$ & $1.5 \mathrm{E}-02$ \\
1575 & $0.0 \mathrm{E}+00$ & $8.5 \mathrm{E}-09$ & $2.3 \mathrm{E}-03$ & $1.0 \mathrm{E}-02$ & $3.0 \mathrm{E}-03$ & $1.5 \mathrm{E}-02$ \\
1750 & $0.0 \mathrm{E}+00$ & $8.5 \mathrm{E}-09$ & $1.6 \mathrm{E}-03$ & $7.1 \mathrm{E}-03$ & $2.1 \mathrm{E}-03$ & $1.1 \mathrm{E}-02$ \\
2175 & $0.0 \mathrm{E}+00$ & $8.3 \mathrm{E}-09$ & $5.2 \mathrm{E}-05$ & $2.3 \mathrm{E}-04$ & $6.7 \mathrm{E}-05$ & $3.5 \mathrm{E}-04$ \\
4000 & $0.0 \mathrm{E}+00$ & $8.1 \mathrm{E}-09$ & $5.3 \mathrm{E}-05$ & $2.4 \mathrm{E}-04$ & $6.9 \mathrm{E}-05$ & $3.6 \mathrm{E}-04$ \\
6000 & $0.0 \mathrm{E}+00$ & $8.0 \mathrm{E}-09$ & $5.4 \mathrm{E}-05$ & $2.6 \mathrm{E}-04$ & $7.7 \mathrm{E}-05$ & $3.9 \mathrm{E}-04$ \\
8000 & $0.0 \mathrm{E}+00$ & $7.8 \mathrm{E}-09$ & $5.7 \mathrm{E}-05$ & $2.5 \mathrm{E}-04$ & $7.4 \mathrm{E}-05$ & $3.8 \mathrm{E}-04$ \\
10000 & $0.0 \mathrm{E}+00$ & $7.7 \mathrm{E}-09$ & $6.0 \mathrm{E}-05$ & $2.6 \mathrm{E}-04$ & $7.7 \mathrm{E}-05$ & $4.0 \mathrm{E}-04$ \\
& & & & & & \\
\hline
\end{tabular}


GBLRM Cancer Risk from Drinking Water in Zone B Due to Groundwater Release

\begin{tabular}{|c|c|c|c|}
\hline Time (yr) & As & $\mathrm{Cr}$ & $\mathrm{Ni}$ \\
\hline 15 & $0.0 E+00$ & $0.0 \mathrm{E}+00$ & $0.0 \mathrm{E}+00$ \\
\hline 20 & $0.0 \mathrm{E}+00$ & $0.0 E+00$ & $0.0 \mathrm{E}+00$ \\
\hline 25 & $0.0 \mathrm{E}+00$ & $0.0 \mathrm{E}+00$ & $0.0 \mathrm{E}+00$ \\
\hline 30 & $0.0 \mathrm{E}+00$ & $0.0 E+00$ & $0.0 E+00$ \\
\hline 60 & $0.0 \mathrm{E}+00$ & $0.0 \mathrm{E}+00$ & $0.0 \mathrm{E}+00$ \\
\hline 62 & $0.0 E+00$ & $0.0 E+00$ & $0.0 E+00$ \\
\hline 80 & $0.0 E+00$ & $0.0 \mathrm{E}+00$ & $0.0 \mathrm{E}+00$ \\
\hline 100 & $0.0 E+00$ & $0.0 \mathrm{E}+00$ & $0.0 \mathrm{E}+00$ \\
\hline 125 & $0.0 E+00$ & $0.0 E+00$ & $0.0 E+00$ \\
\hline 575 & $0.0 E+00$ & $0.0 \mathrm{E}+00$ & $0.0 \mathrm{E}+00$ \\
\hline 590 & $0.0 E+00$ & $0.0 E+00$ & $0.0 \mathrm{E}+00$ \\
\hline 620 & $0.0 E+00$ & $0.0 E+00$ & $0.0 E+00$ \\
\hline 750 & $0.0 E+00$ & $0.0 \mathrm{E}+00$ & $0.0 \mathrm{E}+00$ \\
\hline 1000 & $0.0 E+00$ & $0.0 E+00$ & $0.0 E+00$ \\
\hline 1015 & $0.0 \mathrm{E}+00$ & $0.0 E+00$ & $0.0 \mathrm{E}+00$ \\
\hline 1025 & $0.0 \mathrm{E}+00$ & $0.0 E+00$ & $0.0 E+00$ \\
\hline 1060 & $0.0 \mathrm{E}+00$ & $0.0 E+00$ & $0.0 E+00$ \\
\hline 1080 & $0.0 \mathrm{E}+00$ & $0.0 E+00$ & $0.0 E+00$ \\
\hline 1120 & $0.0 \mathrm{E}+00$ & $0.0 E+00$ & $0.0 E+00$ \\
\hline 1175 & $0.0 \mathrm{E}+00$ & $0.0 E+00$ & $0.0 E+00$ \\
\hline 1575 & $0.0 E+00$ & $0.0 \mathrm{E}+00$ & $0.0 E+00$ \\
\hline 1750 & $0.0 \mathrm{E}+00$ & $0.0 E+00$ & $0.0 \mathrm{E}+00$ \\
\hline 2175 & $0.0 \mathrm{E}+00$ & $0.0 E+00$ & $0.0 \mathrm{E}+00$ \\
\hline 4000 & $0.0 \mathrm{E}+00$ & $0.0 E+00$ & $0.0 E+00$ \\
\hline 6000 & $5.1 E-03$ & $0.0 \mathrm{E}+00$ & $0.0 \mathrm{E}+00$ \\
\hline 8000 & $3.1 E-02$ & $0.0 \mathrm{E}+00$ & $0.0 \mathrm{E}+00$ \\
\hline 10000 & $3.1 E-02$ & $0.0 \mathrm{E}+00$ & $0.0 \mathrm{E}+00$ \\
\hline
\end{tabular}

AAVCU Concentration in Lettuce (Bq $\mathrm{kg}^{-1}$ ) in Zone $\mathrm{A}$ Due to Atmospheric Release

\begin{tabular}{rrrrrr}
\hline Time (yr) & $\mathrm{U}-238 \& 4$ & $\mathrm{Th}-230$ & $\mathrm{Ra}-226$ & $\mathrm{~Pb}-210$ & PO-210 \\
\hline 1 & $2.7 \mathrm{E}-02$ & $2.2 \mathrm{E}-01$ & $2.2 \mathrm{E}-01$ & $2.2 \mathrm{E}-01$ & $2.2 \mathrm{E}-01$ \\
20 & $2.8 \mathrm{E}-02$ & $2.2 \mathrm{E}-01$ & $2.4 \mathrm{E}-01$ & $2.2 \mathrm{E}-01$ & $2.2 \mathrm{E}-01$ \\
25 & $2.8 \mathrm{E}-02$ & $2.2 \mathrm{E}-01$ & $2.5 \mathrm{E}-01$ & $2.2 \mathrm{E}-01$ & $2.2 \mathrm{E}-01$ \\
30 & $2.8 \mathrm{E}-02$ & $2.2 \mathrm{E}-01$ & $2.5 \mathrm{E}-01$ & $2.2 \mathrm{E}-01$ & $2.2 \mathrm{E}-01$ \\
62 & $2.8 \mathrm{E}-02$ & $2.2 \mathrm{E}-01$ & $2.8 \mathrm{E}-01$ & $2.2 \mathrm{E}-01$ & $2.3 \mathrm{E}-01$ \\
125 & $2.8 \mathrm{E}-02$ & $2.2 \mathrm{E}-01$ & $3.2 \mathrm{E}-01$ & $2.2 \mathrm{E}-01$ & $2.3 \mathrm{E}-01$ \\
575 & $2.9 \mathrm{E}-02$ & $2.2 \mathrm{E}-01$ & $3.8 \mathrm{E}-01$ & $2.3 \mathrm{E}-01$ & $2.4 \mathrm{E}-01$ \\
750 & $2.9 \mathrm{E}-02$ & $2.2 \mathrm{E}-01$ & $3.9 \mathrm{E}-01$ & $2.3 \mathrm{E}-01$ & $2.4 \mathrm{E}-01$ \\
1000 & $2.9 \mathrm{E}-02$ & $2.2 \mathrm{E}-01$ & $3.9 \mathrm{E}-01$ & $2.3 \mathrm{E}-01$ & $2.4 \mathrm{E}-01$ \\
1025 & $1.4 \mathrm{E}-03$ & $1.1 \mathrm{E}-03$ & $1.4 \mathrm{E}-01$ & $5.6 \mathrm{E}-03$ & $1.4 \mathrm{E}-02$ \\
1050 & $1.2 \mathrm{E}-03$ & $9.4 \mathrm{E}-04$ & $1.2 \mathrm{E}-01$ & $4.7 \mathrm{E}-03$ & $1.2 \mathrm{E}-02$ \\
1100 & $8.2 \mathrm{E}-04$ & $6.6 \mathrm{E}-04$ & $8.3 \mathrm{E}-02$ & $3.3 \mathrm{E}-03$ & $8.3 \mathrm{E}-03$ \\
1200 & $4.1 \mathrm{E}-04$ & $3.3 \mathrm{E}-04$ & $4.1 \mathrm{E}-02$ & $1.7 \mathrm{E}-03$ & $4.1 \mathrm{E}-03$ \\
1400 & $1.0 \mathrm{E}-04$ & $8.3 \mathrm{E}-05$ & $1.0 \mathrm{E}-02$ & $4.1 \mathrm{E}-04$ & $1.0 \mathrm{E}-03$ \\
1600 & $2.6 \mathrm{E}-05$ & $2.1 \mathrm{E}-05$ & $2.6 \mathrm{E}-03$ & $1.0 \mathrm{E}-04$ & $2.6 \mathrm{E}-04$ \\
2000 & $1.6 \mathrm{E}-06$ & $1.3 \mathrm{E}-06$ & $1.6 \mathrm{E}-04$ & $6.5 \mathrm{E}-06$ & $1.6 \mathrm{E}-05$ \\
2500 & $5.0 \mathrm{E}-08$ & $4.0 \mathrm{E}-08$ & $5.0 \mathrm{E}-06$ & $2.0 \mathrm{E}-07$ & $5.0 \mathrm{E}-07$ \\
3000 & $1.6 \mathrm{E}-09$ & $1.2 \mathrm{E}-09$ & $1.6 \mathrm{E}-07$ & $6.3 \mathrm{E}-09$ & $1.6 \mathrm{E}-08$ \\
& & & & & \\
\hline
\end{tabular}


AAVDU Dose from Lettuce Ingestion in Zone A Due to Atmospheric Release (Sv $\mathrm{yr}^{-1}$ )

\begin{tabular}{rrrrrrr}
\hline Time (yr) & U-238\&4 & Th-230 & Ra-226 & Pb-210 & Po-210 & Chain \\
\hline 1 & $2.8 \mathrm{E}-08$ & $1.9 \mathrm{E}-06$ & $1.2 \mathrm{E}-06$ & $7.2 \mathrm{E}-06$ & $3.4 \mathrm{E}-06$ & $1.4 \mathrm{E}-05$ \\
20 & $2.8 \mathrm{E}-08$ & $1.9 \mathrm{E}-06$ & $1.3 \mathrm{E}-06$ & $7.2 \mathrm{E}-06$ & $3.5 \mathrm{E}-06$ & $1.4 \mathrm{E}-05$ \\
25 & $2.8 \mathrm{E}-08$ & $1.9 \mathrm{E}-06$ & $1.4 \mathrm{E}-06$ & $7.2 \mathrm{E}-06$ & $3.5 \mathrm{E}-06$ & $1.4 \mathrm{E}-05$ \\
30 & $2.8 \mathrm{E}-08$ & $1.9 \mathrm{E}-06$ & $1.4 \mathrm{E}-06$ & $7.2 \mathrm{E}-06$ & $3.5 \mathrm{E}-06$ & $1.4 \mathrm{E}-05$ \\
62 & $2.8 \mathrm{E}-08$ & $1.9 \mathrm{E}-06$ & $1.5 \mathrm{E}-06$ & $7.3 \mathrm{E}-06$ & $3.5 \mathrm{E}-06$ & $1.4 \mathrm{E}-05$ \\
125 & $2.9 \mathrm{E}-08$ & $1.9 \mathrm{E}-06$ & $1.7 \mathrm{E}-06$ & $7.3 \mathrm{E}-06$ & $3.6 \mathrm{E}-06$ & $1.5 \mathrm{E}-05$ \\
575 & $2.9 \mathrm{E}-08$ & $1.9 \mathrm{E}-06$ & $2.1 \mathrm{E}-06$ & $7.4 \mathrm{E}-06$ & $3.7 \mathrm{E}-06$ & $1.5 \mathrm{E}-05$ \\
750 & $2.9 \mathrm{E}-08$ & $1.9 \mathrm{E}-06$ & $2.1 \mathrm{E}-06$ & $7.4 \mathrm{E}-06$ & $3.7 \mathrm{E}-06$ & $1.5 \mathrm{E}-05$ \\
1000 & $2.9 \mathrm{E}-08$ & $1.9 \mathrm{E}-06$ & $2.1 \mathrm{E}-06$ & $7.4 \mathrm{E}-06$ & $3.7 \mathrm{E}-06$ & $1.5 \mathrm{E}-05$ \\
1025 & $1.4 \mathrm{E}-09$ & $9.8 \mathrm{E}-09$ & $7.7 \mathrm{E}-07$ & $1.8 \mathrm{E}-07$ & $2.2 \mathrm{E}-07$ & $1.2 \mathrm{E}-06$ \\
1050 & $1.2 \mathrm{E}-09$ & $8.2 \mathrm{E}-09$ & $6.5 \mathrm{E}-07$ & $1.5 \mathrm{E}-07$ & $1.8 \mathrm{E}-07$ & $9.9 \mathrm{E}-07$ \\
1100 & $8.3 \mathrm{E}-10$ & $5.8 \mathrm{E}-09$ & $4.6 \mathrm{E}-07$ & $1.1 \mathrm{E}-07$ & $1.3 \mathrm{E}-07$ & $7.0 \mathrm{E}-07$ \\
1200 & $4.2 \mathrm{E}-10$ & $2.9 \mathrm{E}-09$ & $2.3 \mathrm{E}-07$ & $5.4 \mathrm{E}-08$ & $6.4 \mathrm{E}-08$ & $3.5 \mathrm{E}-07$ \\
1400 & $1.0 \mathrm{E}-10$ & $7.2 \mathrm{E}-10$ & $5.7 \mathrm{E}-08$ & $1.3 \mathrm{E}-08$ & $1.6 \mathrm{E}-08$ & $8.7 \mathrm{E}-08$ \\
1600 & $2.6 \mathrm{E}-11$ & $1.8 \mathrm{E}-10$ & $1.4 \mathrm{E}-08$ & $3.4 \mathrm{E}-09$ & $4.0 \mathrm{E}-09$ & $2.2 \mathrm{E}-08$ \\
2000 & $1.6 \mathrm{E}-12$ & $1.1 \mathrm{E}-11$ & $8.9 \mathrm{E}-10$ & $2.1 \mathrm{E}-10$ & $2.5 \mathrm{E}-10$ & $1.4 \mathrm{E}-09$ \\
2500 & $5.1 \mathrm{E}-14$ & $3.5 \mathrm{E}-13$ & $2.8 \mathrm{E}-11$ & $6.6 \mathrm{E}-12$ & $7.8 \mathrm{E}-12$ & $4.3 \mathrm{E}-11$ \\
3000 & $1.6 \mathrm{E}-15$ & $1.1 \mathrm{E}-14$ & $8.7 \mathrm{E}-13$ & $2.0 \mathrm{E}-13$ & $2.4 \mathrm{E}-13$ & $1.3 \mathrm{E}-12$ \\
\hline
\end{tabular}

AAVRU Cancer Risk from Lettuce Ingestion in Zone A Due to Atmospheric Release

\begin{tabular}{rrrrrrr}
\hline Time $(y r)$ & U-238\&4 & Th-230 & Ra-226 & Pb-210 & Po-210 & Chain \\
\hline 1 & $2.9 \mathrm{E}-08$ & $1.4 \mathrm{E}-07$ & $1.2 \mathrm{E}-06$ & $1.6 \mathrm{E}-06$ & $5.4 \mathrm{E}-06$ & $8.4 \mathrm{E}-06$ \\
20 & $2.9 \mathrm{E}-08$ & $1.4 \mathrm{E}-07$ & $1.4 \mathrm{E}-06$ & $1.6 \mathrm{E}-06$ & $5.4 \mathrm{E}-06$ & $8.6 \mathrm{E}-06$ \\
25 & $2.9 \mathrm{E}-08$ & $1.4 \mathrm{E}-07$ & $1.4 \mathrm{E}-06$ & $1.6 \mathrm{E}-06$ & $5.4 \mathrm{E}-06$ & $8.6 \mathrm{E}-06$ \\
30 & $2.9 \mathrm{E}-08$ & $1.4 \mathrm{E}-07$ & $1.4 \mathrm{E}-06$ & $1.6 \mathrm{E}-06$ & $5.4 \mathrm{E}-06$ & $8.6 \mathrm{E}-06$ \\
62 & $2.9 \mathrm{E}-08$ & $1.4 \mathrm{E}-07$ & $1.6 \mathrm{E}-06$ & $1.6 \mathrm{E}-06$ & $5.5 \mathrm{E}-06$ & $8.8 \mathrm{E}-06$ \\
125 & $3.0 \mathrm{E}-08$ & $1.4 \mathrm{E}-07$ & $1.8 \mathrm{E}-06$ & $1.7 \mathrm{E}-06$ & $5.5 \mathrm{E}-06$ & $9.1 \mathrm{E}-06$ \\
575 & $3.0 \mathrm{E}-08$ & $1.4 \mathrm{E}-07$ & $2.2 \mathrm{E}-06$ & $1.7 \mathrm{E}-06$ & $5.5 \mathrm{E}-06$ & $9.6 \mathrm{E}-06$ \\
750 & $3.0 \mathrm{E}-08$ & $1.4 \mathrm{E}-07$ & $2.2 \mathrm{E}-06$ & $1.7 \mathrm{E}-06$ & $5.5 \mathrm{E}-06$ & $9.6 \mathrm{E}-06$ \\
1000 & $3.0 \mathrm{E}-08$ & $1.4 \mathrm{E}-07$ & $2.2 \mathrm{E}-06$ & $1.7 \mathrm{E}-06$ & $5.5 \mathrm{E}-06$ & $9.6 \mathrm{E}-06$ \\
1025 & $1.4 \mathrm{E}-09$ & $6.8 \mathrm{E}-10$ & $7.8 \mathrm{E}-07$ & $1.4 \mathrm{E}-07$ & $1.0 \mathrm{E}-07$ & $1.0 \mathrm{E}-06$ \\
1050 & $1.2 \mathrm{E}-09$ & $5.7 \mathrm{E}-10$ & $6.6 \mathrm{E}-07$ & $1.2 \mathrm{E}-07$ & $8.4 \mathrm{E}-08$ & $8.6 \mathrm{E}-07$ \\
1100 & $8.6 \mathrm{E}-10$ & $4.1 \mathrm{E}-10$ & $4.6 \mathrm{E}-07$ & $8.1 \mathrm{E}-08$ & $6.0 \mathrm{E}-08$ & $6.1 \mathrm{E}-07$ \\
1200 & $4.3 \mathrm{E}-10$ & $2.0 \mathrm{E}-10$ & $2.3 \mathrm{E}-07$ & $4.1 \mathrm{E}-08$ & $3.0 \mathrm{E}-08$ & $3.0 \mathrm{E}-07$ \\
1400 & $1.1 \mathrm{E}-10$ & $5.1 \mathrm{E}-11$ & $5.8 \mathrm{E}-08$ & $1.0 \mathrm{E}-08$ & $7.4 \mathrm{E}-09$ & $7.6 \mathrm{E}-08$ \\
1600 & $2.7 \mathrm{E}-11$ & $1.3 \mathrm{E}-11$ & $1.5 \mathrm{E}-08$ & $2.5 \mathrm{E}-09$ & $1.9 \mathrm{E}-09$ & $1.9 \mathrm{E}-08$ \\
2000 & $1.7 \mathrm{E}-12$ & $7.9 \mathrm{E}-13$ & $9.1 \mathrm{E}-10$ & $1.6 \mathrm{E}-10$ & $1.2 \mathrm{E}-10$ & $1.2 \mathrm{E}-09$ \\
2500 & $5.2 \mathrm{E}-14$ & $2.5 \mathrm{E}-14$ & $2.8 \mathrm{E}-11$ & $4.9 \mathrm{E}-12$ & $3.6 \mathrm{E}-12$ & $3.7 \mathrm{E}-11$ \\
3000 & $1.6 \mathrm{E}-15$ & $7.6 \mathrm{E}-16$ & $8.8 \mathrm{E}-13$ & $1.5 \mathrm{E}-13$ & $1.1 \mathrm{E}-13$ & $1.2 \mathrm{E}-12$ \\
\hline
\end{tabular}


AAVCM Concentration in Lettuce ( $\mathrm{mg} \mathrm{kg}^{-1}$ ) in Zone $A$ Due to Atmospheric Release

\begin{tabular}{rrrr}
\hline Time $(y r)$ & As & $\mathrm{Cr}$ & $\mathrm{Ni}$ \\
\hline 1 & $1.6 \mathrm{E}-03$ & $1.6 \mathrm{E}-04$ & $1.6 \mathrm{E}-05$ \\
20 & $1.6 \mathrm{E}-03$ & $1.6 \mathrm{E}-04$ & $1.6 \mathrm{E}-05$ \\
25 & $1.6 \mathrm{E}-03$ & $1.6 \mathrm{E}-04$ & $1.6 \mathrm{E}-05$ \\
30 & $1.6 \mathrm{E}-03$ & $1.6 \mathrm{E}-04$ & $1.6 \mathrm{E}-05$ \\
62 & $1.6 \mathrm{E}-03$ & $1.7 \mathrm{E}-04$ & $1.6 \mathrm{E}-05$ \\
125 & $1.6 \mathrm{E}-03$ & $1.7 \mathrm{E}-04$ & $1.6 \mathrm{E}-05$ \\
575 & $1.7 \mathrm{E}-03$ & $1.8 \mathrm{E}-04$ & $1.7 \mathrm{E}-05$ \\
750 & $1.7 \mathrm{E}-03$ & $1.8 \mathrm{E}-04$ & $1.7 \mathrm{E}-05$ \\
1000 & $1.7 \mathrm{E}-03$ & $1.8 \mathrm{E}-04$ & $1.7 \mathrm{E}-05$ \\
1025 & $8.0 \mathrm{E}-05$ & $2.0 \mathrm{E}-05$ & $1.0 \mathrm{E}-06$ \\
1050 & $6.7 \mathrm{E}-05$ & $1.7 \mathrm{E}-05$ & $8.4 \mathrm{E}-07$ \\
1100 & $4.8 \mathrm{E}-05$ & $1.2 \mathrm{E}-05$ & $6.0 \mathrm{E}-07$ \\
1200 & $2.4 \mathrm{E}-05$ & $6.0 \mathrm{E}-06$ & $3.0 \mathrm{E}-07$ \\
1400 & $6.0 \mathrm{E}-06$ & $1.5 \mathrm{E}-06$ & $7.4 \mathrm{E}-08$ \\
1600 & $1.5 \mathrm{E}-06$ & $3.7 \mathrm{E}-07$ & $1.9 \mathrm{E}-08$ \\
2000 & $9.3 \mathrm{E}-08$ & $2.3 \mathrm{E}-08$ & $1.2 \mathrm{E}-09$ \\
2500 & $2.9 \mathrm{E}-09$ & $7.3 \mathrm{E}-10$ & $3.6 \mathrm{E}-11$ \\
3000 & $9.1 \mathrm{E}-11$ & $2.3 \mathrm{E}-11$ & $1.1 \mathrm{E}-12$ \\
& & &
\end{tabular}

AAVRM Cancer Risk from Lettuce Ingestion in Zone A Due to Atmospheric Release

\begin{tabular}{rrrr}
\hline Time $(\mathrm{Yr})$ & As & $\mathrm{Cr}$ & $\mathrm{Ni}$ \\
\hline 1 & $6.9 \mathrm{E}-04$ & $0.0 \mathrm{E}+00$ & $0.0 \mathrm{E}+00$ \\
20 & $7.0 \mathrm{E}-04$ & $0.0 \mathrm{E}+00$ & $0.0 \mathrm{E}+00$ \\
25 & $7.0 \mathrm{E}-04$ & $0.0 \mathrm{E}+00$ & $0.0 \mathrm{E}+00$ \\
30 & $7.0 \mathrm{E}-04$ & $0.0 \mathrm{E}+00$ & $0.0 \mathrm{E}+00$ \\
62 & $7.1 \mathrm{E}-04$ & $0.0 \mathrm{E}+00$ & $0.0 \mathrm{E}+00$ \\
125 & $7.1 \mathrm{E}-04$ & $0.0 \mathrm{E}+00$ & $0.0 \mathrm{E}+00$ \\
575 & $7.3 \mathrm{E}-04$ & $0.0 \mathrm{E}+00$ & $0.0 \mathrm{E}+00$ \\
750 & $7.3 \mathrm{E}-04$ & $0.0 \mathrm{E}+00$ & $0.0 \mathrm{E}+00$ \\
1000 & $7.3 \mathrm{E}-04$ & $0.0 \mathrm{E}+00$ & $0.0 \mathrm{E}+00$ \\
1025 & $3.5 \mathrm{E}-05$ & $0.0 \mathrm{E}+00$ & $0.0 \mathrm{E}+00$ \\
1050 & $2.9 \mathrm{E}-05$ & $0.0 \mathrm{E}+00$ & $0.0 \mathrm{E}+00$ \\
1100 & $2.1 \mathrm{E}-05$ & $0.0 \mathrm{E}+00$ & $0.0 \mathrm{E}+00$ \\
1200 & $1.0 \mathrm{E}-05$ & $0.0 \mathrm{E}+00$ & $0.0 \mathrm{E}+00$ \\
1400 & $2.6 \mathrm{E}-06$ & $0.0 \mathrm{E}+00$ & $0.0 \mathrm{E}+00$ \\
1600 & $6.5 \mathrm{E}-07$ & $0.0 \mathrm{E}+00$ & $0.0 \mathrm{E}+00$ \\
2000 & $4.1 \mathrm{E}-08$ & $0.0 \mathrm{E}+00$ & $0.0 \mathrm{E}+00$ \\
2500 & $1.3 \mathrm{E}-09$ & $0.0 \mathrm{E}+00$ & $0.0 \mathrm{E}+00$ \\
3000 & $4.0 \mathrm{E}-11$ & $0.0 \mathrm{E}+00$ & $0.0 \mathrm{E}+00$ \\
& & & \\
\hline
\end{tabular}

AAVIM Intake from Lettuce Ingestion in Zone A Due to Atmospheric Release (mg $\mathrm{yr}^{-1}$ )

\begin{tabular}{rrrr}
\hline Time $(y r)$ & As & Cr & Ni \\
\hline 1 & $3.9 E-02$ & $4.0 E-03$ & $3.9 E-04$ \\
25 & $4.0 E-02$ & $4.0 E-03$ & $4.0 E-04$ \\
30 & $4.0 E-02$ & $4.0 E-03$ & $4.0 E-04$ \\
62 & $4.0 E-02$ & $4.1 E-03$ & $4.0 E-04$ \\
125 & $4.1 E-02$ & $4.2 E-03$ & $4.0 E-04$ \\
575 & $4.2 E-02$ & $4.5 E-03$ & $4.1 E-04$ \\
750 & $4.2 E-02$ & $4.5 E-03$ & $4.2 E-04$ \\
1000 & $4.2 E-02$ & $4.5 E-03$ & $4.2 E-04$ \\
1025 & $2.0 E-03$ & $5.0 E-04$ & $2.5 E-05$ \\
1050 & $1.7 E-03$ & $4.2 E-04$ & $2.1 E-05$ \\
1100 & $1.2 E-03$ & $3.0 E-04$ & $1.5 E-05$ \\
1200 & $6.0 E-04$ & $1.5 E-04$ & $7.4 E-06$ \\
1400 & $1.5 E-04$ & $3.7 E-05$ & $1.9 E-06$ \\
1600 & $3.7 E-05$ & $9.3 E-06$ & $4.6 E-07$ \\
2000 & $2.3 E-06$ & $5.8 E-07$ & $2.9 E-08$ \\
2500 & $7.3 E-08$ & $1.8 E-08$ & $9.1 E-10$ \\
3000 & $2.3 E-09$ & $5.7 E-10$ & $2.8 E-11$
\end{tabular}


ABVCU Concentration in Lettuce ( $\mathrm{Bq} \mathrm{kg}{ }^{-1}$ ) in zone $B$ Due to Atmospheric Release

\begin{tabular}{rccccc}
\hline Time $(\mathrm{Yr})$ & $\mathrm{U}-238 \& 4$ & Th-230 & Ra-226 & Pb-210 & Po-210 \\
\hline 1 & $5.0 \mathrm{E}-03$ & $4.0 \mathrm{E}-02$ & $4.0 \mathrm{E}-02$ & $4.0 \mathrm{E}-02$ & $4.0 \mathrm{E}-02$ \\
20 & $5.0 \mathrm{E}-03$ & $4.0 \mathrm{E}-02$ & $4.4 \mathrm{E}-02$ & $4.0 \mathrm{E}-02$ & $4.0 \mathrm{E}-02$ \\
25 & $5.0 \mathrm{E}-03$ & $4.0 \mathrm{E}-02$ & $4.5 \mathrm{E}-02$ & $4.0 \mathrm{E}-02$ & $4.1 \mathrm{E}-02$ \\
30 & $5.0 \mathrm{E}-03$ & $4.0 \mathrm{E}-02$ & $4.6 \mathrm{E}-02$ & $4.0 \mathrm{E}-02$ & $4.1 \mathrm{E}-02$ \\
62 & $5.1 \mathrm{E}-03$ & $4.0 \mathrm{E}-02$ & $5.1 \mathrm{E}-02$ & $4.1 \mathrm{E}-02$ & $4.1 \mathrm{E}-02$ \\
125 & $5.1 \mathrm{E}-03$ & $4.0 \mathrm{E}-02$ & $5.8 \mathrm{E}-02$ & $4.1 \mathrm{E}-02$ & $4.2 \mathrm{E}-02$ \\
575 & $5.3 \mathrm{E}-03$ & $4.0 \mathrm{E}-02$ & $7.0 \mathrm{E}-02$ & $4.1 \mathrm{E}-02$ & $4.3 \mathrm{E}-02$ \\
750 & $5.3 \mathrm{E}-03$ & $4.0 \mathrm{E}-02$ & $7.0 \mathrm{E}-02$ & $4.1 \mathrm{E}-02$ & $4.3 \mathrm{E}-02$ \\
1000 & $5.3 \mathrm{E}-03$ & $4.0 \mathrm{E}-02$ & $7.0 \mathrm{E}-02$ & $4.1 \mathrm{E}-02$ & $4.3 \mathrm{E}-02$ \\
1025 & $2.5 \mathrm{E}-04$ & $2.0 \mathrm{E}-04$ & $2.5 \mathrm{E}-02$ & $1.0 \mathrm{E}-03$ & $2.5 \mathrm{E}-03$ \\
1050 & $2.1 \mathrm{E}-04$ & $1.7 \mathrm{E}-04$ & $2.1 \mathrm{E}-02$ & $8.6 \mathrm{E}-04$ & $2.1 \mathrm{E}-03$ \\
1100 & $1.5 \mathrm{E}-04$ & $1.2 \mathrm{E}-04$ & $1.5 \mathrm{E}-02$ & $6.0 \mathrm{E}-04$ & $1.5 \mathrm{E}-03$ \\
1200 & $7.5 \mathrm{E}-05$ & $6.0 \mathrm{E}-05$ & $7.5 \mathrm{E}-03$ & $3.0 \mathrm{E}-04$ & $7.5 \mathrm{E}-04$ \\
1400 & $1.9 \mathrm{E}-05$ & $1.5 \mathrm{E}-05$ & $1.9 \mathrm{E}-03$ & $7.6 \mathrm{E}-05$ & $1.9 \mathrm{E}-04$ \\
1600 & $4.7 \mathrm{E}-06$ & $3.7 \mathrm{E}-06$ & $4.7 \mathrm{E}-04$ & $1.9 \mathrm{E}-05$ & $4.7 \mathrm{E}-05$ \\
2000 & $2.9 \mathrm{E}-07$ & $2.3 \mathrm{E}-07$ & $2.9 \mathrm{E}-05$ & $1.2 \mathrm{E}-06$ & $2.9 \mathrm{E}-06$ \\
2500 & $9.2 \mathrm{E}-09$ & $7.3 \mathrm{E}-09$ & $9.2 \mathrm{E}-07$ & $3.7 \mathrm{E}-08$ & $9.2 \mathrm{E}-08$ \\
3000 & $2.9 \mathrm{E}-10$ & $2.3 \mathrm{E}-10$ & $2.9 \mathrm{E}-08$ & $1.1 \mathrm{E}-09$ & $2.9 \mathrm{E}-09$ \\
\hline
\end{tabular}

ABVDU Dose from Lettuce Ingestion in Zone $B$ Due to Atmospheric Release (Sv $\mathrm{yr}^{-1}$ )

\begin{tabular}{rcccccc}
\hline Time (yr) & U-238\&4 & Th-230 & Ra-226 & Pb-210 & Po-210 & Chain \\
\hline 1 & $5.0 \mathrm{E}-09$ & $3.5 \mathrm{E}-07$ & $2.2 \mathrm{E}-07$ & $1.3 \mathrm{E}-06$ & $6.2 \mathrm{E}-07$ & $2.5 \mathrm{E}-06$ \\
20 & $5.1 \mathrm{E}-09$ & $3.5 \mathrm{E}-07$ & $2.4 \mathrm{E}-07$ & $1.3 \mathrm{E}-06$ & $6.3 \mathrm{E}-07$ & $2.5 \mathrm{E}-06$ \\
25 & $5.1 \mathrm{E}-09$ & $3.5 \mathrm{E}-07$ & $2.5 \mathrm{E}-07$ & $1.3 \mathrm{E}-06$ & $6.3 \mathrm{E}-07$ & $2.5 \mathrm{E}-06$ \\
30 & $5.1 \mathrm{E}-09$ & $3.5 \mathrm{E}-07$ & $2.5 \mathrm{E}-07$ & $1.3 \mathrm{E}-06$ & $6.3 \mathrm{E}-07$ & $2.5 \mathrm{E}-06$ \\
62 & $5.1 \mathrm{E}-09$ & $3.5 \mathrm{E}-07$ & $2.8 \mathrm{E}-07$ & $1.3 \mathrm{E}-06$ & $6.4 \mathrm{E}-07$ & $2.6 \mathrm{E}-06$ \\
125 & $5.2 \mathrm{E}-09$ & $3.5 \mathrm{E}-07$ & $3.2 \mathrm{E}-07$ & $1.3 \mathrm{E}-06$ & $6.5 \mathrm{E}-07$ & $2.6 \mathrm{E}-06$ \\
575 & $5.3 \mathrm{E}-09$ & $3.5 \mathrm{E}-07$ & $3.8 \mathrm{E}-07$ & $1.3 \mathrm{E}-06$ & $6.7 \mathrm{E}-07$ & $2.7 \mathrm{E}-06$ \\
750 & $5.3 \mathrm{E}-09$ & $3.5 \mathrm{E}-07$ & $3.9 \mathrm{E}-07$ & $1.3 \mathrm{E}-06$ & $6.7 \mathrm{E}-07$ & $2.7 \mathrm{E}-06$ \\
1000 & $5.3 \mathrm{E}-09$ & $3.5 \mathrm{E}-07$ & $3.9 \mathrm{E}-07$ & $1.3 \mathrm{E}-06$ & $6.7 \mathrm{E}-07$ & $2.7 \mathrm{E}-06$ \\
1025 & $2.5 \mathrm{E}-10$ & $1.8 \mathrm{E}-09$ & $1.4 \mathrm{E}-07$ & $3.3 \mathrm{E}-08$ & $3.9 \mathrm{E}-08$ & $2.1 \mathrm{E}-07$ \\
1050 & $2.1 \mathrm{E}-10$ & $1.5 \mathrm{E}-09$ & $1.2 \mathrm{E}-07$ & $2.8 \mathrm{E}-08$ & $3.3 \mathrm{E}-08$ & $1.8 \mathrm{E}-07$ \\
1100 & $1.5 \mathrm{E}-10$ & $1.1 \mathrm{E}-09$ & $8.3 \mathrm{E}-08$ & $2.0 \mathrm{E}-08$ & $2.3 \mathrm{E}-08$ & $1.3 \mathrm{E}-07$ \\
1200 & $7.6 \mathrm{E}-11$ & $5.3 \mathrm{E}-10$ & $4.1 \mathrm{E}-08$ & $9.8 \mathrm{E}-09$ & $1.2 \mathrm{E}-08$ & $6.4 \mathrm{E}-08$ \\
1400 & $1.9 \mathrm{E}-11$ & $1.3 \mathrm{E}-10$ & $1.0 \mathrm{E}-08$ & $2.4 \mathrm{E}-09$ & $2.9 \mathrm{E}-09$ & $1.6 \mathrm{E}-08$ \\
1600 & $4.7 \mathrm{E}-12$ & $3.3 \mathrm{E}-11$ & $2.6 \mathrm{E}-09$ & $6.1 \mathrm{E}-10$ & $7.3 \mathrm{E}-10$ & $4.0 \mathrm{E}-09$ \\
2000 & $3.0 \mathrm{E}-13$ & $2.0 \mathrm{E}-12$ & $1.6 \mathrm{E}-10$ & $3.8 \mathrm{E}-11$ & $4.6 \mathrm{E}-11$ & $2.5 \mathrm{E}-10$ \\
2500 & $9.2 \mathrm{E}-15$ & $6.3 \mathrm{E}-14$ & $5.0 \mathrm{E}-12$ & $1.2 \mathrm{E}-12$ & $1.4 \mathrm{E}-12$ & $7.7 \mathrm{E}-12$ \\
3000 & $2.9 \mathrm{E}-16$ & $2.0 \mathrm{E}-15$ & $1.6 \mathrm{E}-13$ & $3.7 \mathrm{E}-14$ & $4.4 \mathrm{E}-14$ & $2.4 \mathrm{E}-13$ \\
& & & & & & \\
\hline
\end{tabular}


ABVRU Cancer Risk from Lettuce Ingestion in Zone B Due to Atmospheric Release

\begin{tabular}{rrrrrrr}
\hline Time (yr) & $\mathrm{U}-238 \& 4$ & Th-230 & Ra-226 & Pb-210 & Po-210 & Chain \\
\hline 1 & $5.2 \mathrm{E}-09$ & $2.5 \mathrm{E}-08$ & $2.3 \mathrm{E}-07$ & $2.9 \mathrm{E}-07$ & $9.8 \mathrm{E}-07$ & $1.5 \mathrm{E}-06$ \\
20 & $5.2 \mathrm{E}-09$ & $2.5 \mathrm{E}-08$ & $2.5 \mathrm{E}-07$ & $2.9 \mathrm{E}-07$ & $9.8 \mathrm{E}-07$ & $1.6 \mathrm{E}-06$ \\
25 & $5.2 \mathrm{E}-09$ & $2.5 \mathrm{E}-08$ & $2.5 \mathrm{E}-07$ & $2.9 \mathrm{E}-07$ & $9.9 \mathrm{E}-07$ & $1.6 \mathrm{E}-06$ \\
30 & $5.2 \mathrm{E}-09$ & $2.5 \mathrm{E}-08$ & $2.6 \mathrm{E}-07$ & $2.9 \mathrm{E}-07$ & $9.9 \mathrm{E}-07$ & $1.6 \mathrm{E}-06$ \\
62 & $5.3 \mathrm{E}-09$ & $2.5 \mathrm{E}-08$ & $2.8 \mathrm{E}-07$ & $3.0 \mathrm{E}-07$ & $9.9 \mathrm{E}-07$ & $1.6 \mathrm{E}-06$ \\
125 & $5.4 \mathrm{E}-09$ & $2.5 \mathrm{E}-08$ & $3.2 \mathrm{E}-07$ & $3.0 \mathrm{E}-07$ & $9.9 \mathrm{E}-07$ & $1.7 \mathrm{E}-06$ \\
575 & $5.5 \mathrm{E}-09$ & $2.5 \mathrm{E}-08$ & $3.9 \mathrm{E}-07$ & $3.2 \mathrm{E}-07$ & $1.0 \mathrm{E}-06$ & $1.7 \mathrm{E}-06$ \\
750 & $5.5 \mathrm{E}-09$ & $2.5 \mathrm{E}-08$ & $3.9 \mathrm{E}-07$ & $3.2 \mathrm{E}-07$ & $1.0 \mathrm{E}-06$ & $1.7 \mathrm{E}-06$ \\
1000 & $5.5 \mathrm{E}-09$ & $2.4 \mathrm{E}-08$ & $3.9 \mathrm{E}-07$ & $3.2 \mathrm{E}-07$ & $1.0 \mathrm{E}-06$ & $1.7 \mathrm{E}-06$ \\
1025 & $2.6 \mathrm{E}-10$ & $1.2 \mathrm{E}-10$ & $1.4 \mathrm{E}-07$ & $2.5 \mathrm{E}-08$ & $1.8 \mathrm{E}-08$ & $1.9 \mathrm{E}-07$ \\
1050 & $2.2 \mathrm{E}-10$ & $1.0 \mathrm{E}-10$ & $1.2 \mathrm{E}-07$ & $2.1 \mathrm{E}-08$ & $1.5 \mathrm{E}-08$ & $1.6 \mathrm{E}-07$ \\
1100 & $1.6 \mathrm{E}-10$ & $7.4 \mathrm{E}-11$ & $8.4 \mathrm{E}-08$ & $1.5 \mathrm{E}-08$ & $1.1 \mathrm{E}-08$ & $1.1 \mathrm{E}-07$ \\
1200 & $7.8 \mathrm{E}-11$ & $3.7 \mathrm{E}-11$ & $4.2 \mathrm{E}-08$ & $7.4 \mathrm{E}-09$ & $5.4 \mathrm{E}-09$ & $5.5 \mathrm{E}-08$ \\
1400 & $1.9 \mathrm{E}-11$ & $9.2 \mathrm{E}-12$ & $1.1 \mathrm{E}-08$ & $1.8 \mathrm{E}-09$ & $1.4 \mathrm{E}-09$ & $1.4 \mathrm{E}-08$ \\
1600 & $4.9 \mathrm{E}-12$ & $2.3 \mathrm{E}-12$ & $2.6 \mathrm{E}-09$ & $4.6 \mathrm{E}-10$ & $3.4 \mathrm{E}-10$ & $3.4 \mathrm{E}-09$ \\
2000 & $3.0 \mathrm{E}-13$ & $1.4 \mathrm{E}-13$ & $1.6 \mathrm{E}-10$ & $2.9 \mathrm{E}-11$ & $2.1 \mathrm{E}-11$ & $2.1 \mathrm{E}-10$ \\
2500 & $9.5 \mathrm{E}-15$ & $4.4 \mathrm{E}-15$ & $5.1 \mathrm{E}-12$ & $9.0 \mathrm{E}-13$ & $6.6 \mathrm{E}-13$ & $6.7 \mathrm{E}-12$ \\
3000 & $3.0 \mathrm{E}-16$ & $1.4 \mathrm{E}-16$ & $1.6 \mathrm{E}-13$ & $2.8 \mathrm{E}-14$ & $2.1 \mathrm{E}-14$ & $2.1 \mathrm{E}-13$ \\
\hline
\end{tabular}

ABVCM Concentration in Lettuce ( $\mathrm{mg} \mathrm{kg}^{-1}$ ) in Zone $\mathrm{B}$ Due to Atmospheric Release

\begin{tabular}{rrrr}
\hline Time (yr) & As & Cr & $N i$ \\
\hline 1 & $2.9 \mathrm{E}-04$ & $2.9 \mathrm{E}-05$ & $2.9 \mathrm{E}-06$ \\
20 & $2.9 \mathrm{E}-04$ & $2.9 \mathrm{E}-05$ & $2.9 \mathrm{E}-06$ \\
25 & $2.9 \mathrm{E}-04$ & $2.9 \mathrm{E}-05$ & $2.9 \mathrm{E}-06$ \\
30 & $2.9 \mathrm{E}-04$ & $2.9 \mathrm{E}-05$ & $2.9 \mathrm{E}-06$ \\
62 & $2.9 \mathrm{E}-04$ & $3.0 \mathrm{E}-05$ & $2.9 \mathrm{E}-06$ \\
125 & $3.0 \mathrm{E}-04$ & $3.1 \mathrm{E}-05$ & $3.0 \mathrm{E}-06$ \\
575 & $3.0 \mathrm{E}-04$ & $3.3 \mathrm{E}-05$ & $3.1 \mathrm{E}-06$ \\
750 & $3.0 \mathrm{E}-04$ & $3.3 \mathrm{E}-05$ & $3.1 \mathrm{E}-06$ \\
1000 & $3.0 \mathrm{E}-04$ & $3.3 \mathrm{E}-05$ & $3.1 \mathrm{E}-06$ \\
1025 & $1.4 \mathrm{E}-05$ & $3.6 \mathrm{E}-06$ & $1.8 \mathrm{E}-07$ \\
1050 & $1.2 \mathrm{E}-05$ & $3.0 \mathrm{E}-06$ & $1.5 \mathrm{E}-07$ \\
1100 & $8.6 \mathrm{E}-06$ & $2.2 \mathrm{E}-06$ & $1.1 \mathrm{E}-07$ \\
1200 & $4.3 \mathrm{E}-06$ & $1.1 \mathrm{E}-06$ & $5.4 \mathrm{E}-08$ \\
1400 & $1.1 \mathrm{E}-06$ & $2.7 \mathrm{E}-07$ & $1.3 \mathrm{E}-08$ \\
1600 & $2.7 \mathrm{E}-07$ & $6.7 \mathrm{E}-08$ & $3.4 \mathrm{E}-09$ \\
2000 & $1.7 \mathrm{E}-08$ & $4.2 \mathrm{E}-09$ & $2.1 \mathrm{E}-10$ \\
2500 & $5.2 \mathrm{E}-10$ & $1.3 \mathrm{E}-10$ & $6.6 \mathrm{E}-12$ \\
3000 & $1.6 \mathrm{E}-11$ & $4.1 \mathrm{E}-12$ & $2.1 \mathrm{E}-13$
\end{tabular}

ABVIM Intake from Lettuce Ingestion in Zone $B$ Due to Atmospheric Release (mg $\mathrm{yr}^{-1}$ )

\begin{tabular}{rrrrr}
\hline Time $(Y r)$ & As & $\mathrm{Cr}$ & $\mathrm{Ni}$ \\
\hline 1 & $7.2 \mathrm{E}-03$ & $7.2 \mathrm{E}-04$ & $7.2 \mathrm{E}-05$ \\
20 & $7.2 \mathrm{E}-03$ & $7.3 \mathrm{E}-04$ & $7.2 \mathrm{E}-05$ \\
25 & $7.2 \mathrm{E}-03$ & $7.3 \mathrm{E}-04$ & $7.2 \mathrm{E}-05$ \\
30 & $7.2 \mathrm{E}-03$ & $7.4 \mathrm{E}-04$ & $7.3 \mathrm{E}-05$ \\
62 & $7.3 \mathrm{E}-03$ & $7.5 \mathrm{E}-04$ & $7.3 \mathrm{E}-05$ \\
125 & $7.4 \mathrm{E}-03$ & $7.8 \mathrm{E}-04$ & $7.5 \mathrm{E}-05$ \\
575 & $7.6 \mathrm{E}-03$ & $8.2 \mathrm{E}-04$ & $7.7 \mathrm{E}-05$ \\
750 & $7.6 \mathrm{E}-03$ & $8.2 \mathrm{E}-04$ & $7.6 \mathrm{E}-05$ \\
1000 & $7.6 \mathrm{E}-03$ & $8.2 \mathrm{E}-04$ & $7.6 \mathrm{E}-05$ \\
1025 & $3.6 \mathrm{E}-04$ & $9.0 \mathrm{E}-05$ & $4.5 \mathrm{E}-06$ \\
1050 & $3.0 \mathrm{E}-04$ & $7.6 \mathrm{E}-05$ & $3.8 \mathrm{E}-06$ \\
1100 & $2.2 \mathrm{E}-04$ & $5.4 \mathrm{E}-05$ & $2.7 \mathrm{E}-06$ \\
1200 & $1.1 \mathrm{E}-04$ & $2.7 \mathrm{E}-05$ & $1.3 \mathrm{E}-06$ \\
1400 & $2.7 \mathrm{E}-05$ & $6.7 \mathrm{E}-06$ & $3.4 \mathrm{E}-07$ \\
1600 & $6.7 \mathrm{E}-06$ & $1.7 \mathrm{E}-06$ & $8.4 \mathrm{E}-08$ \\
2000 & $4.2 \mathrm{E}-07$ & $1.0 \mathrm{E}-07$ & $5.2 \mathrm{E}-09$ \\
2500 & $1.3 \mathrm{E}-08$ & $3.3 \mathrm{E}-09$ & $1.6 \mathrm{E}-10$ \\
3000 & $4.1 \mathrm{E}-10$ & $1.0 \mathrm{E}-10$ & $5.1 \mathrm{E}-12$
\end{tabular}


ABVRM Cancer Risk from Lettuce Ingestion in Zone $B$ Due to Atmospheric Release

\begin{tabular}{rrrr}
\hline Time $(y r)$ & As & $\mathrm{Cr}$ & $\mathrm{Ni}$ \\
\hline 1 & $1.3 \mathrm{E}-04$ & $0.0 \mathrm{E}+00$ & $0.0 \mathrm{E}+00$ \\
20 & $1.3 \mathrm{E}-04$ & $0.0 \mathrm{E}+00$ & $0.0 \mathrm{E}+00$ \\
25 & $1.3 \mathrm{E}-04$ & $0.0 \mathrm{E}+00$ & $0.0 \mathrm{E}+00$ \\
30 & $1.3 \mathrm{E}-04$ & $0.0 \mathrm{E}+00$ & $0.0 \mathrm{E}+00$ \\
62 & $1.3 \mathrm{E}-04$ & $0.0 \mathrm{E}+00$ & $0.0 \mathrm{E}+00$ \\
125 & $1.3 \mathrm{E}-04$ & $0.0 \mathrm{E}+00$ & $0.0 \mathrm{E}+00$ \\
575 & $1.3 \mathrm{E}-04$ & $0.0 \mathrm{E}+00$ & $0.0 \mathrm{E}+00$ \\
750 & $1.3 \mathrm{E}-04$ & $0.0 \mathrm{E}+00$ & $0.0 \mathrm{E}+00$ \\
1000 & $1.3 \mathrm{E}-04$ & $0.0 \mathrm{E}+00$ & $0.0 \mathrm{E}+00$ \\
1025 & $6.3 \mathrm{E}-06$ & $0.0 \mathrm{E}+00$ & $0.0 \mathrm{E}+00$ \\
1050 & $5.3 \mathrm{E}-06$ & $0.0 \mathrm{E}+00$ & $0.0 \mathrm{E}+00$ \\
1100 & $3.8 \mathrm{E}-06$ & $0.0 \mathrm{E}+00$ & $0.0 \mathrm{E}+00$ \\
1200 & $1.9 \mathrm{E}-06$ & $0.0 \mathrm{E}+00$ & $0.0 \mathrm{E}+00$ \\
1400 & $4.7 \mathrm{E}-07$ & $0.0 \mathrm{E}+00$ & $0.0 \mathrm{E}+00$ \\
1600 & $1.2 \mathrm{E}-07$ & $0.0 \mathrm{E}+00$ & $0.0 \mathrm{E}+00$ \\
2000 & $7.3 \mathrm{E}-09$ & $0.0 \mathrm{E}+00$ & $0.0 \mathrm{E}+00$ \\
2500 & $2.3 \mathrm{E}-10$ & $0.0 \mathrm{E}+00$ & $0.0 \mathrm{E}+00$ \\
3000 & $7.2 \mathrm{E}-12$ & $0.0 \mathrm{E}+00$ & $0.0 \mathrm{E}+00$ \\
\hline
\end{tabular}

GAVCU Concentration in Lettuce ( $\mathrm{Bq} \mathrm{kg}^{-1}$ ) in Zone $\mathrm{A}$ Due to Groundwater Release

\begin{tabular}{|c|c|c|c|c|c|}
\hline$(y r)$ & $U-238 \& 4$ & $T h-230$ & $R a-226$ & $\mathrm{~Pb}-210$ & Po- 210 \\
\hline 15 & $0.0 E+00$ & $0.0 E+00$ & $0.0 \mathrm{E}+00$ & $0.0 \mathrm{E}+00$ & $0.0 E+00$ \\
\hline 20 & $0.0 E+00$ & $0.0 E+00$ & $0.0 \mathrm{E}+00$ & $0.0 E+00$ & $0.0 \mathrm{E}+00$ \\
\hline 25 & $0.0 \mathrm{E}+00$ & $0.0 E+00$ & $0.0 E+00$ & $0.0 E+00$ & $0.0 E+00$ \\
\hline 30 & $0.0 \mathrm{E}+00$ & $0.0 E+00$ & $0.0 \mathrm{E}+00$ & $0.0 \mathrm{E}+00$ & $0.0 E+00$ \\
\hline 60 & $0.0 E+00$ & $0.0 E+00$ & $0.0 E+00$ & $0.0 \mathrm{E}+00$ & $0.0 \mathrm{E}+00$ \\
\hline 62 & $1.1 E-01$ & $4.2 \mathrm{E}-10$ & $9.9 \mathrm{E}-11$ & $9.1 \mathrm{E}-11$ & $9.1 E-11$ \\
\hline 80 & $5.9 \mathrm{E}+00$ & $1.3 E-06$ & $7.8 E-08$ & $7.3 E-08$ & $7.3 E-08$ \\
\hline 100 & 1. $2 \mathrm{E}+01$ & $5.6 E-06$ & $6.9 \mathrm{E}-07$ & $6.4 \mathrm{E}-07$ & $6.4 \mathrm{E}-07$ \\
\hline 125 & 2. $0 E+01$ & $1.5 E-05$ & $3.1 \mathrm{E}-06$ & $2.9 \mathrm{E}-06$ & $2.9 E-06$ \\
\hline 575 & 2. $0 E+01$ & $2.3 E-04$ & $4.9 E-04$ & $4.6 \mathrm{E}-04$ & $4.6 E-04$ \\
\hline 590 & $2.0 E+01$ & $2.3 E-04$ & $8.8 E-02$ & $8.9 \mathrm{E}-02$ & $8.9 E-02$ \\
\hline 620 & 2. $0 E+01$ & $2.5 E-04$ & $8.7 E-01$ & $8.7 \mathrm{E}-01$ & $8.8 E-01$ \\
\hline 750 & $2.0 E+01$ & 3. $1 \mathrm{E}-04$ & $4.2 E+00$ & $4.2 E+00$ & $4.2 E+00$ \\
\hline 1000 & 2. $0 E+01$ & $4.3 E-04$ & $1.0 E+01$ & 1. $0 \mathrm{E}+01$ & $1.0 E+01$ \\
\hline 1015 & $2.0 E+01$ & $4.4 \mathrm{E}-04$ & 1. $0 \mathrm{E}+01$ & 1. $0 E+01$ & 1. $0 E+01$ \\
\hline 1025 & 2. $0 \mathrm{E}+01$ & $4.4 \mathrm{E}-04$ & $1.1 \mathrm{E}+01$ & $1.1 E+01$ & 1. $1 \mathrm{E}+01$ \\
\hline 1060 & $2.0 E+01$ & $4.6 \mathrm{E}-04$ & 1. $1 \mathrm{E}+01$ & $1.1 E+01$ & 1. $1 E+01$ \\
\hline 1080 & 2. $0 E+01$ & $4.7 \mathrm{E}-04$ & 1. $2 E+01$ & 1. $2 \mathrm{E}+01$ & 1. $2 E+01$ \\
\hline 1120 & 1. $1 E+00$ & $4.7 \mathrm{E}-04$ & 1. $3 E+01$ & 1. $3 E+01$ & 1. $3 E+01$ \\
\hline 1175 & $0.0 \mathrm{E}+00$ & $4.7 \mathrm{E}-04$ & 1. $4 \mathrm{E}+01$ & 1. $4 \mathrm{E}+01$ & 1. $4 E+01$ \\
\hline 1575 & $0.0 E+00$ & $4.7 E-04$ & $1.4 E+01$ & $1.4 \mathrm{E}+01$ & 1. $4 E+01$ \\
\hline 1750 & $0.0 E+00$ & 4. $7 \mathrm{E}-04$ & $9.8 E+00$ & $9.7 E+00$ & $9.7 E+00$ \\
\hline 2175 & $0.0 \mathrm{E}+00$ & $4.6 E-04$ & 3. $0 E-01$ & 3. $0 E-01$ & 4. $0 \mathrm{E}-01$ \\
\hline 4000 & $0.0 \mathrm{E}+00$ & $4.5 \mathrm{E}-04$ & $4.0 \mathrm{E}-01$ & 3. $0 \mathrm{E}-01$ & 3. $0 \mathrm{E}-01$ \\
\hline 6000 & $0.0 E+00$ & $4.4 \mathrm{E}-04$ & $3.0 E-01$ & $4.0 E-01$ & $4.0 E-01$ \\
\hline 8000 & $0.0 E \div 00$ & $4.3 E-04$ & $4.0 \mathrm{E}-01$ & $3.0 \mathrm{E}-01$ & 4. $0 \mathrm{E}-01$ \\
\hline 10000 & $0.0 \mathrm{E}+00$ & 4. $2 E-04$ & $3.0 \mathrm{E}-01$ & $3.0 E-01$ & $3.0 E-01$ \\
\hline
\end{tabular}


GBVCU Concentration in Lettuce (Bq $\mathrm{kg}^{-1}$ ) in Zone B Due to Groundwater Release

\begin{tabular}{|c|c|c|c|c|c|}
\hline Time (yr) & $U-238 \& 4$ & Th-230 & $\mathrm{Ra}-226$ & $\mathrm{~Pb}-210$ & Po- 210 \\
\hline 15 & $0.0 \mathrm{E}+00$ & $0.0 E+00$ & $0.0 \mathrm{E}+00$ & $0.0 E+00$ & $0.0 E+00$ \\
\hline 20 & $0.0 \mathrm{E}+00$ & $0.0 \mathrm{E}+00$ & $0.0 \mathrm{E}+00$ & $0.0 E+00$ & $0.0 \mathrm{E} \div 00$ \\
\hline 25 & $0.0 E+00$ & $0.0 E+00$ & $0.0 \mathrm{E}+00$ & $0.0 E+00$ & $0.0 \mathrm{E}+00$ \\
\hline 30 & $0.0 E+00$ & $0.0 E+00$ & $0.0 \mathrm{E}+00$ & $0.0 E+00$ & $0.0 E \div 00$ \\
\hline 60 & $0.0 E+00$ & $0.0 E+00$ & $0.0 \mathrm{E}+00$ & $0.0 E+00$ & $0.0 E+00$ \\
\hline 62 & 1. $3 E-02$ & $5.0 E-11$ & 1. $2 \mathrm{E}-11$ & $1.1 E-11$ & 1. $1 \mathrm{E}-11$ \\
\hline 80 & $7.0 \mathrm{E}-01$ & $1.5 \mathrm{E}-07$ & $9.3 E-09$ & $8.6 E-09$ & $8.6 \mathrm{E}-09$ \\
\hline 100 & 1. $5 E+00$ & $6.7 E-07$ & $8.2 \mathrm{E}-08$ & $7.6 E-08$ & $7.6 \mathrm{E}-08$ \\
\hline 125 & 2. $3 E+00$ & $1.8 \mathrm{E}-06$ & $3.7 \mathrm{E}-07$ & $3.5 E-07$ & $3.5 E-07$ \\
\hline 575 & $2.3 E+00$ & $2.7 \mathrm{E}-05$ & $5.9 E-05$ & $5.4 E-05$ & $5.4 E-05$ \\
\hline 590 & $2.3 E+00$ & $2.8 \mathrm{E}-05$ & 1. $1 \mathrm{E}-02$ & 1. $1 E-02$ & 1. $1 \mathrm{E}-02$ \\
\hline 620 & 2. $3 E+00$ & $2.9 \mathrm{E}-05$ & 1. $0 \mathrm{E}-01$ & 1. $0 \mathrm{E}-01$ & 1. $0 E-01$ \\
\hline 750 & $2.3 E+00$ & $3.7 \mathrm{E}-05$ & $5.0 \mathrm{E}-01$ & $5.0 E-01$ & $5.0 \mathrm{E}-01$ \\
\hline 1000 & $2.3 E+00$ & $5.1 E-05$ & 1. $2 \mathrm{E}+00$ & 1. $2 E+00$ & 1. $2 \mathrm{E}+00$ \\
\hline 1015 & $2.3 E+00$ & $5.2 \mathrm{E}-05$ & 1. $2 \mathrm{E}+00$ & 1. $2 E+00$ & 1. $2 E+00$ \\
\hline 1025 & $2.3 E+00$ & $5.3 E-05$ & 1. $3 E+00$ & 1. $3 E+00$ & 1. $3 \mathrm{E}+00$ \\
\hline 1060 & $2.3 E+00$ & $5.5 E-05$ & 1. $4 \mathrm{E}+00$ & 1. $4 E+00$ & 1. $4 \mathrm{E} \div 00$ \\
\hline 1080 & $2.3 E+00$ & $5.6 \mathrm{E}-05$ & 1. $4 \mathrm{E}+00$ & 1. $4 E+00$ & $1.4 \mathrm{E}+00$ \\
\hline 1120 & 1. $2 \mathrm{E}-01$ & $5.6 E-05$ & 1. $5 E+00$ & 1. $5 E+00$ & 1. $5 \mathrm{E} \div 00$ \\
\hline 1175 & $0.0 \mathrm{E}+00$ & $5.6 E-05$ & $1.6 \mathrm{E}+00$ & 1. $6 E+00$ & 1. $6 \mathrm{E}+00$ \\
\hline 1575 & $0.0 \mathrm{E}+00$ & $5.6 \mathrm{E}-05$ & 1. $6 \mathrm{E}+00$ & 1. $6 E+00$ & $1.6 \mathrm{E}+00$ \\
\hline 1750 & $0.0 E+00$ & $5.5 E-05$ & 1. $2 E+00$ & 1. $2 E+00$ & 1. $2 E \div 00$ \\
\hline 2175 & $0.0 \mathrm{E}+00$ & $5.4 \mathrm{E}-05$ & $4.0 \mathrm{E}-02$ & $4.0 E-02$ & 3. $0 \mathrm{E}-02$ \\
\hline 4000 & $0.0 E+00$ & $5.3 E-05$ & $4.0 \mathrm{E}-02$ & $4.0 E-02$ & $4.0 \mathrm{E}-02$ \\
\hline 6000 & $0.0 E+00$ & $5.3 E-05$ & $4.0 \mathrm{E}-02$ & $4.0 E-02$ & $4.0 \mathrm{E}-02$ \\
\hline 8000 & $0.0 E+00$ & $5.1 E-05$ & 4. $0 \mathrm{E}-02$ & 4. $0 E-02$ & 4. $0 E-02$ \\
\hline 10000 & $0.0 \mathrm{E}+00$ & $5.0 \mathrm{E}-05$ & $4.0 \mathrm{E}-02$ & $4.0 E-02$ & 4. $0 \mathrm{E}-02$ \\
\hline
\end{tabular}


GAVDU Dose from Lettuce Ingestion in Zone A Due to Groundwater Release (Sv $\mathrm{Yr}^{-1}$ )

\begin{tabular}{rllllll}
\hline Time (Yr) & $\mathrm{U}-238 \& 4$ & $\mathrm{Th}-230$ & $\mathrm{Ra}-226$ & $\mathrm{~Pb}-210$ & Po-210 & Chain \\
\hline 15 & $0.0 \mathrm{E}+00$ & $0.0 \mathrm{E}+00$ & $0.0 \mathrm{E}+00$ & $0.0 \mathrm{E}+00$ & $0.0 \mathrm{E}+00$ & $0.0 \mathrm{E}+00$ \\
20 & $0.0 \mathrm{E}+00$ & $0.0 \mathrm{E}+00$ & $0.0 \mathrm{E}+00$ & $0.0 \mathrm{E}+00$ & $0.0 \mathrm{E}+00$ & $0.0 \mathrm{E}+00$ \\
25 & $0.0 \mathrm{E}+00$ & $0.0 \mathrm{E}+00$ & $0.0 \mathrm{E}+00$ & $0.0 \mathrm{E}+00$ & $0.0 \mathrm{E}+00$ & $0.0 \mathrm{E}+00$ \\
30 & $0.0 \mathrm{E}+00$ & $0.0 \mathrm{E}+00$ & $0.0 \mathrm{E}+00$ & $0.0 \mathrm{E}+00$ & $0.0 \mathrm{E}+00$ & $0.0 \mathrm{E}+00$ \\
60 & $0.0 \mathrm{E}+00$ & $0.0 \mathrm{E}+00$ & $0.0 \mathrm{E}+00$ & $0.0 \mathrm{E}+00$ & $0.0 \mathrm{E}+00$ & $0.0 \mathrm{E}+00$ \\
62 & $1.1 \mathrm{E}-07$ & $3.7 \mathrm{E}-15$ & $5.4 \mathrm{E}-16$ & $3.0 \mathrm{E}-15$ & $1.4 \mathrm{E}-15$ & $1.1 \mathrm{E}-07$ \\
80 & $5.9 \mathrm{E}-06$ & $1.1 \mathrm{E}-11$ & $4.3 \mathrm{E}-13$ & $2.4 \mathrm{E}-12$ & $1.1 \mathrm{E}-12$ & $5.9 \mathrm{E}-06$ \\
100 & $1.2 \mathrm{E}-05$ & $4.9 \mathrm{E}-11$ & $3.8 \mathrm{E}-12$ & $2.1 \mathrm{E}-11$ & $9.9 \mathrm{E}-12$ & $1.2 \mathrm{E}-05$ \\
125 & $2.0 \mathrm{E}-05$ & $1.3 \mathrm{E}-10$ & $1.7 \mathrm{E}-11$ & $9.4 \mathrm{E}-11$ & $4.5 \mathrm{E}-11$ & $2.0 \mathrm{E}-05$ \\
575 & $2.0 \mathrm{E}-05$ & $2.0 \mathrm{E}-09$ & $2.7 \mathrm{E}-09$ & $1.5 \mathrm{E}-08$ & $7.1 \mathrm{E}-09$ & $2.0 \mathrm{E}-05$ \\
590 & $2.0 \mathrm{E}-05$ & $2.0 \mathrm{E}-09$ & $4.9 \mathrm{E}-07$ & $2.9 \mathrm{E}-06$ & $1.4 \mathrm{E}-06$ & $2.5 \mathrm{E}-05$ \\
620 & $2.0 \mathrm{E}-05$ & $2.2 \mathrm{E}-09$ & $4.8 \mathrm{E}-06$ & $2.8 \mathrm{E}-05$ & $1.4 \mathrm{E}-05$ & $6.7 \mathrm{E}-05$ \\
750 & $2.0 \mathrm{E}-05$ & $2.7 \mathrm{E}-09$ & $2.3 \mathrm{E}-05$ & $1.4 \mathrm{E}-04$ & $6.5 \mathrm{E}-05$ & $2.4 \mathrm{E}-04$ \\
1000 & $2.0 \mathrm{E}-05$ & $3.8 \mathrm{E}-09$ & $5.5 \mathrm{E}-05$ & $3.3 \mathrm{E}-04$ & $1.6 \mathrm{E}-04$ & $5.6 \mathrm{E}-04$ \\
1015 & $2.0 \mathrm{E}-05$ & $3.8 \mathrm{E}-09$ & $5.7 \mathrm{E}-05$ & $3.4 \mathrm{E}-04$ & $1.6 \mathrm{E}-04$ & $5.7 \mathrm{E}-04$ \\
1025 & $2.0 \mathrm{E}-05$ & $3.9 \mathrm{E}-09$ & $5.8 \mathrm{E}-05$ & $3.4 \mathrm{E}-04$ & $1.6 \mathrm{E}-04$ & $5.9 \mathrm{E}-04$ \\
1060 & $2.0 \mathrm{E}-05$ & $4.0 \mathrm{E}-09$ & $6.2 \mathrm{E}-05$ & $3.7 \mathrm{E}-04$ & $1.8 \mathrm{E}-04$ & $6.3 \mathrm{E}-04$ \\
1080 & $2.0 \mathrm{E}-05$ & $4.1 \mathrm{E}-09$ & $6.5 \mathrm{E}-05$ & $3.8 \mathrm{E}-04$ & $1.8 \mathrm{E}-04$ & $6.5 \mathrm{E}-04$ \\
1120 & $1.1 \mathrm{E}-06$ & $4.1 \mathrm{E}-09$ & $6.9 \mathrm{E}-05$ & $4.1 \mathrm{E}-04$ & $2.0 \mathrm{E}-04$ & $6.8 \mathrm{E}-04$ \\
1175 & $0.0 \mathrm{E}+00$ & $4.1 \mathrm{E}-09$ & $7.5 \mathrm{E}-05$ & $4.5 \mathrm{E}-04$ & $2.1 \mathrm{E}-04$ & $7.4 \mathrm{E}-04$ \\
1575 & $0.0 \mathrm{E}+00$ & $4.1 \mathrm{E}-09$ & $7.6 \mathrm{E}-05$ & $4.5 \mathrm{E}-04$ & $2.1 \mathrm{E}-04$ & $7.4 \mathrm{E}-04$ \\
1750 & $0.0 \mathrm{E}+00$ & $4.1 \mathrm{E}-09$ & $5.4 \mathrm{E}-05$ & $3.2 \mathrm{E}-04$ & $1.5 \mathrm{E}-04$ & $5.2 \mathrm{E}-04$ \\
2175 & $0.0 \mathrm{E}+00$ & $4.0 \mathrm{E}-09$ & $1.8 \mathrm{E}-06$ & $1.1 \mathrm{E}-05$ & $5.0 \mathrm{E}-06$ & $1.8 \mathrm{E}-05$ \\
4000 & $0.0 \mathrm{E}+00$ & $3.9 \mathrm{E}-09$ & $1.7 \mathrm{E}-06$ & $1.1 \mathrm{E}-05$ & $5.0 \mathrm{E}-06$ & $1.8 \mathrm{E}-05$ \\
6000 & $0.0 \mathrm{E}+00$ & $3.9 \mathrm{E}-09$ & $1.8 \mathrm{E}-06$ & $1.2 \mathrm{E}-05$ & $6.0 \mathrm{E}-06$ & $2.0 \mathrm{E}-05$ \\
8000 & $0.0 \mathrm{E}+00$ & $3.8 \mathrm{E}-09$ & $1.9 \mathrm{E}-06$ & $1.1 \mathrm{E}-05$ & $5.0 \mathrm{E}-06$ & $1.8 \mathrm{E}-05$ \\
10000 & $0.0 \mathrm{E}+00$ & $3.8 \mathrm{E}-09$ & $2.0 \mathrm{E}-06$ & $1.2 \mathrm{E}-05$ & $5.0 \mathrm{E}-06$ & $1.9 \mathrm{E}-05$ \\
& & & & & & \\
\hline
\end{tabular}


GBVDU Dose from Lettuce Ingestion in Zone $B$ Due to Groundwater Release (Sv $\mathrm{Yr}^{-1}$ )

\begin{tabular}{rcccccc}
\hline Time (Yr) & U-238\&4 & Th-230 & Ra-226 & Pb-210 & PO-210 & Chain \\
\hline 15 & $0.0 \mathrm{E}+00$ & $0.0 \mathrm{E}+00$ & $0.0 \mathrm{E}+00$ & $0.0 \mathrm{E}+00$ & $0.0 \mathrm{E}+00$ & $0.0 \mathrm{E}+00$ \\
20 & $0.0 \mathrm{E}+00$ & $0.0 \mathrm{E}+00$ & $0.0 \mathrm{E}+00$ & $0.0 \mathrm{E}+00$ & $0.0 \mathrm{E}+00$ & $0.0 \mathrm{E}+00$ \\
25 & $0.0 \mathrm{E}+00$ & $0.0 \mathrm{E}+00$ & $0.0 \mathrm{E}+00$ & $0.0 \mathrm{E}+00$ & $0.0 \mathrm{E}+00$ & $0.0 \mathrm{E}+00$ \\
30 & $0.0 \mathrm{E}+00$ & $0.0 \mathrm{E}+00$ & $0.0 \mathrm{E}+00$ & $0.0 \mathrm{E}+00$ & $0.0 \mathrm{E}+00$ & $0.0 \mathrm{E}+00$ \\
60 & $0.0 \mathrm{E}+00$ & $0.0 \mathrm{E}+00$ & $0.0 \mathrm{E}+00$ & $0.0 \mathrm{E}+00$ & $0.0 \mathrm{E}+00$ & $0.0 \mathrm{E}+00$ \\
62 & $1.3 \mathrm{E}-08$ & $4.4 \mathrm{E}-16$ & $6.5 \mathrm{E}-17$ & $3.5 \mathrm{E}-16$ & $1.7 \mathrm{E}-16$ & $1.3 \mathrm{E}-08$ \\
80 & $7.1 \mathrm{E}-07$ & $1.3 \mathrm{E}-12$ & $5.1 \mathrm{E}-14$ & $2.8 \mathrm{E}-13$ & $1.3 \mathrm{E}-13$ & $7.1 \mathrm{E}-07$ \\
100 & $1.5 \mathrm{E}-06$ & $5.8 \mathrm{E}-12$ & $4.5 \mathrm{E}-13$ & $2.5 \mathrm{E}-12$ & $1.2 \mathrm{E}-12$ & $1.5 \mathrm{E}-06$ \\
125 & $2.4 \mathrm{E}-06$ & $1.6 \mathrm{E}-11$ & $2.1 \mathrm{E}-12$ & $1.1 \mathrm{E}-11$ & $5.4 \mathrm{E}-12$ & $2.4 \mathrm{E}-06$ \\
575 & $2.4 \mathrm{E}-06$ & $2.4 \mathrm{E}-10$ & $3.2 \mathrm{E}-10$ & $1.8 \mathrm{E}-09$ & $8.4 \mathrm{E}-10$ & $2.4 \mathrm{E}-06$ \\
590 & $2.4 \mathrm{E}-06$ & $2.4 \mathrm{E}-10$ & $5.8 \mathrm{E}-08$ & $3.4 \mathrm{E}-07$ & $1.6 \mathrm{E}-07$ & $2.9 \mathrm{E}-06$ \\
620 & $2.4 \mathrm{E}-06$ & $2.6 \mathrm{E}-10$ & $5.7 \mathrm{E}-07$ & $3.4 \mathrm{E}-06$ & $1.6 \mathrm{E}-06$ & $7.9 \mathrm{E}-06$ \\
750 & $2.4 \mathrm{E}-06$ & $3.2 \mathrm{E}-10$ & $2.7 \mathrm{E}-06$ & $1.6 \mathrm{E}-05$ & $7.7 \mathrm{E}-06$ & $2.9 \mathrm{E}-05$ \\
1000 & $2.4 \mathrm{E}-06$ & $4.5 \mathrm{E}-10$ & $6.6 \mathrm{E}-06$ & $3.9 \mathrm{E}-05$ & $1.9 \mathrm{E}-05$ & $6.6 \mathrm{E}-05$ \\
1015 & $2.4 \mathrm{E}-06$ & $4.6 \mathrm{E}-10$ & $6.8 \mathrm{E}-06$ & $4.0 \mathrm{E}-05$ & $1.9 \mathrm{E}-05$ & $6.8 \mathrm{E}-05$ \\
1025 & $2.4 \mathrm{E}-06$ & $4.6 \mathrm{E}-10$ & $6.9 \mathrm{E}-06$ & $4.1 \mathrm{E}-05$ & $2.0 \mathrm{E}-05$ & $7.0 \mathrm{E}-05$ \\
1060 & $2.4 \mathrm{E}-06$ & $4.8 \mathrm{E}-10$ & $7.4 \mathrm{E}-06$ & $4.4 \mathrm{E}-05$ & $2.1 \mathrm{E}-05$ & $7.5 \mathrm{E}-05$ \\
1080 & $2.4 \mathrm{E}-06$ & $4.9 \mathrm{E}-10$ & $7.7 \mathrm{E}-06$ & $4.6 \mathrm{E}-05$ & $2.2 \mathrm{E}-05$ & $7.7 \mathrm{E}-05$ \\
1120 & $1.3 \mathrm{E}-07$ & $4.9 \mathrm{E}-10$ & $8.3 \mathrm{E}-06$ & $4.9 \mathrm{E}-05$ & $2.3 \mathrm{E}-05$ & $8.1 \mathrm{E}-05$ \\
1175 & $0.0 \mathrm{E}+00$ & $4.9 \mathrm{E}-10$ & $9.0 \mathrm{E}-06$ & $5.3 \mathrm{E}-05$ & $2.5 \mathrm{E}-05$ & $8.8 \mathrm{E}-05$ \\
1575 & $0.0 \mathrm{E}+00$ & $4.9 \mathrm{E}-10$ & $9.1 \mathrm{E}-06$ & $5.4 \mathrm{E}-05$ & $2.6 \mathrm{E}-05$ & $8.8 \mathrm{E}-05$ \\
1750 & $0.0 \mathrm{E}+00$ & $4.9 \mathrm{E}-10$ & $6.4 \mathrm{E}-06$ & $3.8 \mathrm{E}-05$ & $1.8 \mathrm{E}-05$ & $6.2 \mathrm{E}-05$ \\
2175 & $0.0 \mathrm{E}+00$ & $4.8 \mathrm{E}-10$ & $2.0 \mathrm{E}-07$ & $1.2 \mathrm{E}-06$ & $5.0 \mathrm{E}-07$ & $1.9 \mathrm{E}-06$ \\
4000 & $0.0 \mathrm{E}+00$ & $4.7 \mathrm{E}-10$ & $2.1 \mathrm{E}-07$ & $1.3 \mathrm{E}-06$ & $6.0 \mathrm{E}-07$ & $2.1 \mathrm{E}-06$ \\
6000 & $0.0 \mathrm{E}+00$ & $4.6 \mathrm{E}-10$ & $2.1 \mathrm{E}-07$ & $1.4 \mathrm{E}-06$ & $6.0 \mathrm{E}-07$ & $2.2 \mathrm{E}-06$ \\
8000 & $0.0 \mathrm{E}+00$ & $4.4 \mathrm{E}-10$ & $2.7 \mathrm{E}-07$ & $1.3 \mathrm{E}-06$ & $7.0 \mathrm{E}-07$ & $2.3 \mathrm{E}-06$ \\
10000 & $0.0 \mathrm{E}+00$ & $4.4 \mathrm{E}-10$ & $3.0 \mathrm{E}-07$ & $1.4 \mathrm{E}-06$ & $6.0 \mathrm{E}-07$ & $2.3 \mathrm{E}-06$ \\
& & & & & & \\
\hline
\end{tabular}


GAVRU Cancer Risk from Lettuce Ingestion in Zone A Due to Groundwater Release

\begin{tabular}{rllllll}
\hline Time (Yr) & $\mathrm{U}-238 \& 4$ & $\mathrm{Th}-230$ & $\mathrm{Ra}-226$ & $\mathrm{~PB}-210$ & Po-210 & Chain \\
\hline 15 & $0.0 \mathrm{E}+00$ & $0.0 \mathrm{E}+00$ & $0.0 \mathrm{E}+00$ & $0.0 \mathrm{E}+00$ & $0.0 \mathrm{E}+00$ & $0.0 \mathrm{E}+00$ \\
20 & $0.0 \mathrm{E}+00$ & $0.0 \mathrm{E}+00$ & $0.0 \mathrm{E}+00$ & $0.0 \mathrm{E}+00$ & $0.0 \mathrm{E}+00$ & $0.0 \mathrm{E}+00$ \\
25 & $0.0 \mathrm{E}+00$ & $0.0 \mathrm{E}+00$ & $0.0 \mathrm{E}+00$ & $0.0 \mathrm{E}+00$ & $0.0 \mathrm{E}+00$ & $0.0 \mathrm{E}+00$ \\
30 & $0.0 \mathrm{E}+00$ & $0.0 \mathrm{E}+00$ & $0.0 \mathrm{E}+00$ & $0.0 \mathrm{E}+00$ & $0.0 \mathrm{E}+00$ & $0.0 \mathrm{E}+00$ \\
60 & $0.0 \mathrm{E}+00$ & $0.0 \mathrm{E}+00$ & $0.0 \mathrm{E}+00$ & $0.0 \mathrm{E}+00$ & $0.0 \mathrm{E}+00$ & $0.0 \mathrm{E}+00$ \\
62 & $1.1 \mathrm{E}-07$ & $2.6 \mathrm{E}-16$ & $5.5 \mathrm{E}-16$ & $2.2 \mathrm{E}-15$ & $6.6 \mathrm{E}-16$ & $1.1 \mathrm{E}-07$ \\
80 & $6.1 \mathrm{E}-06$ & $7.9 \mathrm{E}-13$ & $4.4 \mathrm{E}-13$ & $1.8 \mathrm{E}-12$ & $5.2 \mathrm{E}-13$ & $6.1 \mathrm{E}-06$ \\
100 & $1.3 \mathrm{E}-05$ & $3.4 \mathrm{E}-12$ & $3.9 \mathrm{E}-12$ & $1.6 \mathrm{E}-11$ & $4.6 \mathrm{E}-12$ & $1.3 \mathrm{E}-05$ \\
125 & $2.1 \mathrm{E}-05$ & $9.4 \mathrm{E}-12$ & $1.8 \mathrm{E}-11$ & $7.1 \mathrm{E}-11$ & $2.1 \mathrm{E}-11$ & $2.1 \mathrm{E}-05$ \\
575 & $2.0 \mathrm{E}-05$ & $1.4 \mathrm{E}-10$ & $2.8 \mathrm{E}-09$ & $1.1 \mathrm{E}-08$ & $3.3 \mathrm{E}-09$ & $2.1 \mathrm{E}-05$ \\
590 & $2.0 \mathrm{E}-05$ & $1.4 \mathrm{E}-10$ & $4.9 \mathrm{E}-07$ & $2.2 \mathrm{E}-06$ & $6.4 \mathrm{E}-07$ & $2.4 \mathrm{E}-05$ \\
620 & $2.0 \mathrm{E}-05$ & $1.5 \mathrm{E}-10$ & $4.9 \mathrm{E}-06$ & $2.1 \mathrm{E}-05$ & $6.3 \mathrm{E}-06$ & $5.3 \mathrm{E}-05$ \\
750 & $2.0 \mathrm{E}-05$ & $1.9 \mathrm{E}-10$ & $2.3 \mathrm{E}-05$ & $1.0 \mathrm{E}-04$ & $3.0 \mathrm{E}-05$ & $1.8 \mathrm{E}-04$ \\
1000 & $2.0 \mathrm{E}-05$ & $2.6 \mathrm{E}-10$ & $5.6 \mathrm{E}-05$ & $2.5 \mathrm{E}-04$ & $7.2 \mathrm{E}-05$ & $3.9 \mathrm{E}-04$ \\
1015 & $2.0 \mathrm{E}-05$ & $2.7 \mathrm{E}-10$ & $5.8 \mathrm{E}-05$ & $2.5 \mathrm{E}-04$ & $7.4 \mathrm{E}-05$ & $4.1 \mathrm{E}-04$ \\
1025 & $2.0 \mathrm{E}-05$ & $2.7 \mathrm{E}-10$ & $5.9 \mathrm{E}-05$ & $2.6 \mathrm{E}-04$ & $7.6 \mathrm{E}-05$ & $4.2 \mathrm{E}-04$ \\
1060 & $2.0 \mathrm{E}-05$ & $2.8 \mathrm{E}-10$ & $5.3 \mathrm{E}-05$ & $2.8 \mathrm{E}-04$ & $8.2 \mathrm{E}-05$ & $4.4 \mathrm{E}-04$ \\
1080 & $2.0 \mathrm{E}-05$ & $2.9 \mathrm{E}-10$ & $6.6 \mathrm{E}-05$ & $2.9 \mathrm{E}-04$ & $8.5 \mathrm{E}-05$ & $4.6 \mathrm{E}-04$ \\
1120 & $1.1 \mathrm{E}-06$ & $2.9 \mathrm{E}-10$ & $7.1 \mathrm{E}-05$ & $3.1 \mathrm{E}-04$ & $9.1 \mathrm{E}-05$ & $4.7 \mathrm{E}-04$ \\
1175 & $1.0 \mathrm{E}-09$ & $2.9 \mathrm{E}-10$ & $7.7 \mathrm{E}-05$ & $3.4 \mathrm{E}-04$ & $9.9 \mathrm{E}-05$ & $5.1 \mathrm{E}-04$ \\
1575 & $1.0 \mathrm{E}-09$ & $2.9 \mathrm{E}-10$ & $7.8 \mathrm{E}-05$ & $3.4 \mathrm{E}-04$ & $1.0 \mathrm{E}-04$ & $5.2 \mathrm{E}-04$ \\
1750 & $1.0 \mathrm{E}-09$ & $2.8 \mathrm{E}-10$ & $5.5 \mathrm{E}-05$ & $2.4 \mathrm{E}-04$ & $7.0 \mathrm{E}-05$ & $3.6 \mathrm{E}-04$ \\
2175 & $1.0 \mathrm{E}-09$ & $2.8 \mathrm{E}-10$ & $1.8 \mathrm{E}-06$ & $7.8 \mathrm{E}-06$ & $2.3 \mathrm{E}-06$ & $1.2 \mathrm{E}-05$ \\
4000 & $0.0 \mathrm{E}+00$ & $2.7 \mathrm{E}-10$ & $1.8 \mathrm{E}-06$ & $8.0 \mathrm{E}-06$ & $2.3 \mathrm{E}-06$ & $1.2 \mathrm{E}-05$ \\
6000 & $1.0 \mathrm{E}-09$ & $2.7 \mathrm{E}-10$ & $1.9 \mathrm{E}-06$ & $8.9 \mathrm{E}-06$ & $2.6 \mathrm{E}-06$ & $1.3 \mathrm{E}-05$ \\
8000 & $1.0 \mathrm{E}-09$ & $2.6 \mathrm{E}-10$ & $1.9 \mathrm{E}-06$ & $8.5 \mathrm{E}-06$ & $2.5 \mathrm{E}-06$ & $1.3 \mathrm{E}-05$ \\
10000 & $1.0 \mathrm{E}-09$ & $2.6 \mathrm{E}-10$ & $2.0 \mathrm{E}-06$ & $8.9 \mathrm{E}-06$ & $2.7 \mathrm{E}-06$ & $1.4 \mathrm{E}-05$ \\
& & & & & & \\
\hline
\end{tabular}


GBVRU Cancer Risk from Lettuce Ingestion in Zone B Due to Groundwater Release

\begin{tabular}{rcccccc}
\hline Time $(y r)$ & $\mathrm{U}-238 \& 4$ & Th-230 & Ra-226 & Pb-210 & Po-210 & Chain \\
\hline 15 & $0.0 \mathrm{E}+00$ & $0.0 \mathrm{E}+00$ & $0.0 \mathrm{E}+00$ & $0.0 \mathrm{E}+00$ & $0.0 \mathrm{E}+00$ & $0.0 \mathrm{E}+00$ \\
20 & $0.0 \mathrm{E}+00$ & $0.0 \mathrm{E}+00$ & $0.0 \mathrm{E}+00$ & $0.0 \mathrm{E}+00$ & $0.0 \mathrm{E}+00$ & $0.0 \mathrm{E}+00$ \\
25 & $0.0 \mathrm{E}+00$ & $0.0 \mathrm{E}+00$ & $0.0 \mathrm{E}+00$ & $0.0 \mathrm{E}+00$ & $0.0 \mathrm{E}+00$ & $0.0 \mathrm{E}+00$ \\
30 & $0.0 \mathrm{E}+00$ & $0.0 \mathrm{E}+00$ & $0.0 \mathrm{E}+00$ & $0.0 \mathrm{E}+00$ & $0.0 \mathrm{E}+00$ & $0.0 \mathrm{E}+00$ \\
60 & $0.0 \mathrm{E}+00$ & $0.0 \mathrm{E}+00$ & $0.0 \mathrm{E}+00$ & $0.0 \mathrm{E}+00$ & $0.0 \mathrm{E}+00$ & $0.0 \mathrm{E}+00$ \\
62 & $1.3 \mathrm{E}-08$ & $3.1 \mathrm{E}-17$ & $6.6 \mathrm{E}-17$ & $2.7 \mathrm{E}-16$ & $7.8 \mathrm{E}-17$ & $1.3 \mathrm{E}-08$ \\
80 & $7.3 \mathrm{E}-07$ & $9.4 \mathrm{E}-14$ & $5.2 \mathrm{E}-14$ & $2.1 \mathrm{E}-13$ & $6.2 \mathrm{E}-14$ & $7.3 \mathrm{E}-07$ \\
100 & $1.5 \mathrm{E}-06$ & $4.1 \mathrm{E}-13$ & $4.6 \mathrm{E}-13$ & $1.9 \mathrm{E}-12$ & $5.5 \mathrm{E}-13$ & $1.5 \mathrm{E}-06$ \\
125 & $2.4 \mathrm{E}-06$ & $1.1 \mathrm{E}-12$ & $2.1 \mathrm{E}-12$ & $8.5 \mathrm{E}-12$ & $2.5 \mathrm{E}-12$ & $2.4 \mathrm{E}-06$ \\
575 & $2.4 \mathrm{E}-06$ & $1.7 \mathrm{E}-11$ & $3.3 \mathrm{E}-10$ & $1.3 \mathrm{E}-09$ & $3.9 \mathrm{E}-10$ & $2.4 \mathrm{E}-06$ \\
590 & $2.4 \mathrm{E}-06$ & $1.7 \mathrm{E}-11$ & $5.9 \mathrm{E}-08$ & $2.6 \mathrm{E}-07$ & $7.6 \mathrm{E}-08$ & $2.8 \mathrm{E}-06$ \\
620 & $2.4 \mathrm{E}-06$ & $1.8 \mathrm{E}-11$ & $5.8 \mathrm{E}-07$ & $2.6 \mathrm{E}-06$ & $7.5 \mathrm{E}-07$ & $6.3 \mathrm{E}-06$ \\
750 & $2.4 \mathrm{E}-06$ & $2.2 \mathrm{E}-11$ & $2.8 \mathrm{E}-06$ & $1.2 \mathrm{E}-05$ & $3.6 \mathrm{E}-06$ & $2.1 \mathrm{E}-05$ \\
1000 & $2.4 \mathrm{E}-06$ & $3.1 \mathrm{E}-11$ & $6.7 \mathrm{E}-06$ & $2.9 \mathrm{E}-05$ & $8.6 \mathrm{E}-06$ & $4.7 \mathrm{E}-05$ \\
1015 & $2.4 \mathrm{E}-06$ & $3.2 \mathrm{E}-11$ & $6.9 \mathrm{E}-06$ & $3.0 \mathrm{E}-05$ & $8.9 \mathrm{E}-06$ & $4.8 \mathrm{E}-05$ \\
1025 & $2.4 \mathrm{E}-06$ & $3.2 \mathrm{E}-11$ & $7.0 \mathrm{E}-06$ & $3.1 \mathrm{E}-05$ & $9.1 \mathrm{E}-06$ & $4.9 \mathrm{E}-05$ \\
1060 & $2.4 \mathrm{E}-06$ & $3.3 \mathrm{E}-11$ & $7.6 \mathrm{E}-06$ & $3.3 \mathrm{E}-05$ & $9.7 \mathrm{E}-06$ & $5.3 \mathrm{E}-05$ \\
1080 & $2.4 \mathrm{E}-06$ & $3.4 \mathrm{E}-11$ & $7.8 \mathrm{E}-06$ & $3.4 \mathrm{E}-05$ & $1.0 \mathrm{E}-05$ & $5.5 \mathrm{E}-05$ \\
1120 & $1.3 \mathrm{E}-07$ & $3.4 \mathrm{E}-11$ & $8.4 \mathrm{E}-06$ & $3.7 \mathrm{E}-05$ & $1.1 \mathrm{E}-05$ & $5.6 \mathrm{E}-05$ \\
1175 & $0.0 \mathrm{E}+00$ & $3.4 \mathrm{E}-11$ & $9.2 \mathrm{E}-06$ & $4.0 \mathrm{E}-05$ & $1.2 \mathrm{E}-05$ & $6.1 \mathrm{E}-05$ \\
1575 & $0.0 \mathrm{E}+00$ & $3.4 \mathrm{E}-11$ & $9.2 \mathrm{E}-06$ & $4.0 \mathrm{E}-05$ & $1.2 \mathrm{E}-05$ & $6.2 \mathrm{E}-05$ \\
1750 & $0.0 \mathrm{E}+00$ & $3.4 \mathrm{E}-11$ & $6.5 \mathrm{E}-06$ & $2.8 \mathrm{E}-05$ & $8.4 \mathrm{E}-06$ & $4.3 \mathrm{E}-05$ \\
2175 & $0.0 \mathrm{E}+00$ & $3.3 \mathrm{E}-11$ & $2.1 \mathrm{E}-07$ & $9.2 \mathrm{E}-07$ & $2.7 \mathrm{E}-07$ & $1.4 \mathrm{E}-06$ \\
4000 & $0.0 \mathrm{E}+00$ & $3.3 \mathrm{E}-11$ & $2.2 \mathrm{E}-07$ & $9.5 \mathrm{E}-07$ & $2.8 \mathrm{E}-07$ & $1.4 \mathrm{E}-06$ \\
6000 & $0.0 \mathrm{E}+00$ & $3.2 \mathrm{E}-11$ & $2.2 \mathrm{E}-07$ & $1.1 \mathrm{E}-06$ & $3.1 \mathrm{E}-07$ & $1.6 \mathrm{E}-06$ \\
8000 & $0.0 \mathrm{E}+00$ & $3.1 \mathrm{E}-11$ & $2.3 \mathrm{E}-07$ & $1.0 \mathrm{E}-06$ & $3.0 \mathrm{E}-07$ & $1.6 \mathrm{E}-06$ \\
10000 & $0.0 \mathrm{E}+00$ & $3.1 \mathrm{E}-11$ & $2.4 \mathrm{E}-07$ & $1.1 \mathrm{E}-06$ & $3.1 \mathrm{E}-07$ & $1.6 \mathrm{E}-06$ \\
& & & & & & \\
\hline
\end{tabular}


GAVCM Concentration in Lettuce (mg $\mathrm{kg}^{-1}$ ) in Zone $\mathrm{A}$ Due to Groundwater Release

\begin{tabular}{rrrr}
\hline Time (Yr) & As & $\mathrm{Cr}$ & $\mathrm{Ni}$ \\
\hline 15 & $0.0 \mathrm{E}+00$ & $3.0 \mathrm{E}-06$ & $3.0 \mathrm{E}-07$ \\
20 & $0.0 \mathrm{E}+00$ & $2.4 \mathrm{E}-01$ & $2.3 \mathrm{E}-02$ \\
25 & $0.0 \mathrm{E}+00$ & $4.7 \mathrm{E}-01$ & $4.7 \mathrm{E}-02$ \\
30 & $0.0 \mathrm{E}+00$ & $7.0 \mathrm{E}-01$ & $7.0 \mathrm{E}-02$ \\
60 & $0.0 \mathrm{E}+00$ & $7.0 \mathrm{E}-01$ & $7.0 \mathrm{E}-02$ \\
62 & $0.0 \mathrm{E}+00$ & $7.0 \mathrm{E}-01$ & $7.0 \mathrm{E}-02$ \\
80 & $0.0 \mathrm{E}+00$ & $7.0 \mathrm{E}-01$ & $7.0 \mathrm{E}-02$ \\
100 & $0.0 \mathrm{E}+00$ & $7.0 \mathrm{E}-01$ & $7.0 \mathrm{E}-02$ \\
125 & $0.0 \mathrm{E}+00$ & $7.0 \mathrm{E}-01$ & $7.0 \mathrm{E}-02$ \\
575 & $0.0 \mathrm{E}+00$ & $7.0 \mathrm{E}-01$ & $7.0 \mathrm{E}-02$ \\
590 & $0.0 \mathrm{E}+00$ & $7.0 \mathrm{E}-01$ & $7.0 \mathrm{E}-02$ \\
620 & $0.0 \mathrm{E}+00$ & $7.0 \mathrm{E}-01$ & $7.0 \mathrm{E}-02$ \\
750 & $0.0 \mathrm{E}+00$ & $7.0 \mathrm{E}-01$ & $7.0 \mathrm{E}-02$ \\
1000 & $0.0 \mathrm{E}+00$ & $7.0 \mathrm{E}-01$ & $7.0 \mathrm{E}-02$ \\
1015 & $0.0 \mathrm{E}+00$ & $7.0 \mathrm{E}-01$ & $7.0 \mathrm{E}-02$ \\
1025 & $0.0 \mathrm{E}+00$ & $2.3 \mathrm{E}-01$ & $2.3 \mathrm{E}-02$ \\
1060 & $0.0 \mathrm{E}+00$ & $0.0 \mathrm{E}+00$ & $0.0 \mathrm{E}+00$ \\
1080 & $0.0 \mathrm{E}+00$ & $0.0 \mathrm{E}+00$ & $0.0 \mathrm{E}+00$ \\
1120 & $0.0 \mathrm{E}+00$ & $0.0 \mathrm{E}+00$ & $0.0 \mathrm{E}+00$ \\
1175 & $0.0 \mathrm{E}+00$ & $0.0 \mathrm{E}+00$ & $0.0 \mathrm{E}+00$ \\
1575 & $0.0 \mathrm{E}+00$ & $0.0 \mathrm{E}+00$ & $0.0 \mathrm{E}+00$ \\
1750 & $0.0 \mathrm{E}+00$ & $0.0 \mathrm{E}+00$ & $0.0 \mathrm{E}+00$ \\
2175 & $0.0 \mathrm{E}+00$ & $0.0 \mathrm{E}+00$ & $0.0 \mathrm{E}+00$ \\
4000 & $0.0 \mathrm{E}+00$ & $0.0 \mathrm{E}+00$ & $0.0 \mathrm{E}+00$ \\
6000 & $3.9 \mathrm{E}-04$ & $0.0 \mathrm{E}+00$ & $0.0 \mathrm{E}+00$ \\
8000 & $2.4 \mathrm{E}-03$ & $0.0 \mathrm{E}+00$ & $0.0 \mathrm{E}+00$ \\
10000 & $2.4 \mathrm{E}-03$ & $0.0 \mathrm{E}+00$ & $0.0 \mathrm{E}+00$ \\
& & & \\
\hline & & &
\end{tabular}

GBVCM Concentration in Lettuce ( $\mathrm{mg} \mathrm{kg}^{-1}$ ) in Zone $\mathrm{B}$ Due to Groundwater Release

\begin{tabular}{rrrr}
\hline Time $(Y r)$ & As & Cr & Ni \\
\hline 15 & $0.0 \mathrm{E}+00$ & $3.6 \mathrm{E}-07$ & $3.6 \mathrm{E}-08$ \\
20 & $0.0 \mathrm{E}+00$ & $2.8 \mathrm{E}-02$ & $2.8 \mathrm{E}-03$ \\
25 & $0.0 \mathrm{E}+00$ & $5.6 \mathrm{E}-02$ & $5.6 \mathrm{E}-03$ \\
30 & $0.0 \mathrm{E}+00$ & $8.4 \mathrm{E}-02$ & $8.4 \mathrm{E}-03$ \\
60 & $0.0 \mathrm{E}+00$ & $8.4 \mathrm{E}-02$ & $8.4 \mathrm{E}-03$ \\
62 & $0.0 \mathrm{E}+00$ & $8.4 \mathrm{E}-02$ & $8.4 \mathrm{E}-03$ \\
80 & $0.0 \mathrm{E}+00$ & $8.4 \mathrm{E}-02$ & $8.4 \mathrm{E}-03$ \\
100 & $0.0 \mathrm{E}+00$ & $8.4 \mathrm{E}-02$ & $8.4 \mathrm{E}-03$ \\
125 & $0.0 \mathrm{E}+00$ & $8.4 \mathrm{E}-02$ & $8.4 \mathrm{E}-03$ \\
575 & $0.0 \mathrm{E}+00$ & $8.4 \mathrm{E}-02$ & $8.3 \mathrm{E}-03$ \\
590 & $0.0 \mathrm{E}+00$ & $8.4 \mathrm{E}-02$ & $8.3 \mathrm{E}-03$ \\
620 & $0.0 \mathrm{E}+00$ & $8.3 \mathrm{E}-02$ & $8.3 \mathrm{E}-03$ \\
750 & $0.0 \mathrm{E}+00$ & $8.3 \mathrm{E}-02$ & $8.3 \mathrm{E}-03$ \\
1000 & $0.0 \mathrm{E}+00$ & $8.3 \mathrm{E}-02$ & $8.3 \mathrm{E}-03$ \\
1015 & $0.0 \mathrm{E}+00$ & $8.3 \mathrm{E}-02$ & $8.3 \mathrm{E}-03$ \\
1025 & $0.0 \mathrm{E}+00$ & $2.8 \mathrm{E}-02$ & $2.8 \mathrm{E}-03$ \\
1060 & $0.0 \mathrm{E}+00$ & $0.0 \mathrm{E}+00$ & $0.0 \mathrm{E}+00$ \\
1080 & $0.0 \mathrm{E}+00$ & $0.0 \mathrm{E}+00$ & $0.0 \mathrm{E}+00$ \\
1120 & $0.0 \mathrm{E}+00$ & $0.0 \mathrm{E}+00$ & $0.0 \mathrm{E}+00$ \\
1175 & $0.0 \mathrm{E}+00$ & $0.0 \mathrm{E}+00$ & $0.0 \mathrm{E}+00$ \\
1575 & $0.0 \mathrm{E}+00$ & $0.0 \mathrm{E}+00$ & $0.0 \mathrm{E}+00$ \\
1750 & $0.0 \mathrm{E}+00$ & $0.0 \mathrm{E}+00$ & $0.0 \mathrm{E}+00$ \\
2175 & $0.0 \mathrm{E}+00$ & $0.0 \mathrm{E}+00$ & $0.0 \mathrm{E}+00$ \\
4000 & $0.0 \mathrm{E}+00$ & $0.0 \mathrm{E}+00$ & $0.0 \mathrm{E}+00$ \\
6000 & $4.7 \mathrm{E}-05$ & $0.0 \mathrm{E}+00$ & $0.0 \mathrm{E}+00$ \\
8000 & $2.9 \mathrm{E}-04$ & $0.0 \mathrm{E}+00$ & $0.0 \mathrm{E}+00$ \\
10000 & $2.8 \mathrm{E}-04$ & $0.0 \mathrm{E}+00$ & $0.0 \mathrm{E}+00$ \\
& & & \\
\hline 100 & &
\end{tabular}


GAVIM Intake from Lettuce Ingestion in Zone A Due to Groundwater Release ( $m g \mathrm{yr}^{-1}$ )

\begin{tabular}{|c|c|c|c|}
\hline Time (yr) & As & $\mathrm{Cr}$ & $\mathrm{Ni}$ \\
\hline 15 & $0.0 E+00$ & $7.5 E-05$ & $7.5 E-06$ \\
\hline 20 & $0.0 E+00$ & $5.9 E+00$ & $5.9 E-01$ \\
\hline 25 & $0.0 \mathrm{E}+00$ & 1. $2 \mathrm{E}+01$ & 1. $2 \mathrm{E}+00$ \\
\hline 30 & $0.0 E+00$ & $1.8 E+01$ & $1.8 \mathrm{E}+00$ \\
\hline 60 & $0.0 \mathrm{E}+00$ & $1.8 \mathrm{E}+01$ & $1.8 E+00$ \\
\hline 62 & $0.0 E+00$ & 1. $8 \mathrm{E}+01$ & 1. $8 \mathrm{E}+00$ \\
\hline 80 & $0.0 \mathrm{E}+00$ & $1.8 E+01$ & 1. $8 \mathrm{E}+00$ \\
\hline 100 & $0.0 E+00$ & $1.8 \mathrm{E}+01$ & $1.8 \mathrm{E}+00$ \\
\hline 125 & $0.0 E+00$ & 1. $8 \mathrm{E}+01$ & $1.8 \mathrm{E}+00$ \\
\hline 575 & $0.0 E+00$ & $1.8 E+01$ & $1.8 \mathrm{E}+00$ \\
\hline 590 & $0.0 E+00$ & $1.8 \mathrm{E}+01$ & $1.8 \mathrm{E}+00$ \\
\hline 620 & $0.0 E+00$ & 1. $8 E+01$ & $1.8 E+00$ \\
\hline 750 & $0.0 \mathrm{E}+00$ & $1.8 E+01$ & $1.7 E+00$ \\
\hline 1000 & $0.0 E+00$ & $1.7 E+01$ & $1.7 E+00$ \\
\hline 1015 & $0.0 E+00$ & 1. $7 \mathrm{E}+01$ & $1.7 \mathrm{E}+00$ \\
\hline 1025 & $0.0 \mathrm{E}+00$ & $5.8 \mathrm{E}+00$ & $5.8 \mathrm{E}-01$ \\
\hline 1060 & $0.0 \mathrm{E}+00$ & $0.0 E+00$ & $0.0 \mathrm{E}+00$ \\
\hline 1080 & $0.0 E+00$ & $0.0 E \div 00$ & $0.0 \mathrm{E}+00$ \\
\hline 1120 & $0.0 \mathrm{E}+00$ & $0.0 \mathrm{E}+00$ & $0.0 \mathrm{E}+00$ \\
\hline 1175 & $0.0 \mathrm{E}+00$ & $0.0 \mathrm{E}+00$ & $0.0 \mathrm{E}+00$ \\
\hline 1575 & $0.0 E+00$ & $0.0 \mathrm{E}+00$ & $0.0 E+00$ \\
\hline 1750 & $0.0 \mathrm{E}+00$ & $0.0 \mathrm{E}+00$ & $0.0 E+00$ \\
\hline 2175 & $0.0 E+00$ & $0.0 \mathrm{E}+00$ & $0.0 E+00$ \\
\hline 4000 & $0.0 \mathrm{E}+00$ & $0.0 \mathrm{E}+00$ & $0.0 E+00$ \\
\hline 6000 & $9.8 E-03$ & $0.0 \mathrm{E}+00$ & $0.0 \mathrm{E}+00$ \\
\hline 8000 & $6.0 \mathrm{E}-02$ & $0.0 E+00$ & $0.0 \mathrm{E}+00$ \\
\hline 10000 & $6.0 \mathrm{E}-02$ & $0.0 E+00$ & $0.0 E+00$ \\
\hline
\end{tabular}

GBVIM Intake from Lettuce Ingestion in Zone $B$ Due to Groundwater Release (mg $\mathrm{yr}^{-1}$ )

\begin{tabular}{rrrr}
\hline Time $(y r)$ & As & Cr & Ni \\
\hline 15 & $0.0 \mathrm{E}+00$ & $8.9 \mathrm{E}-06$ & $8.9 \mathrm{E}-07$ \\
20 & $0.0 \mathrm{E}+00$ & $7.0 \mathrm{E}-01$ & $7.0 \mathrm{E}-02$ \\
25 & $0.0 \mathrm{E}+00$ & $1.4 \mathrm{E}+00$ & $1.4 \mathrm{E}-01$ \\
30 & $0.0 \mathrm{E}+00$ & $2.1 \mathrm{E}+00$ & $2.1 \mathrm{E}-01$ \\
60 & $0.0 \mathrm{E}+00$ & $2.1 \mathrm{E}+00$ & $2.1 \mathrm{E}-01$ \\
62 & $0.0 \mathrm{E}+00$ & $2.1 \mathrm{E}+00$ & $2.1 \mathrm{E}-01$ \\
80 & $0.0 \mathrm{E}+00$ & $2.1 \mathrm{E}+00$ & $2.1 \mathrm{E}-01$ \\
100 & $0.0 \mathrm{E}+00$ & $2.1 \mathrm{E}+00$ & $2.1 \mathrm{E}-01$ \\
125 & $0.0 \mathrm{E}+00$ & $2.1 \mathrm{E}+00$ & $2.1 \mathrm{E}-01$ \\
575 & $0.0 \mathrm{E}+00$ & $2.1 \mathrm{E}+00$ & $2.1 \mathrm{E}-01$ \\
590 & $0.0 \mathrm{E}+00$ & $2.1 \mathrm{E}+00$ & $2.1 \mathrm{E}-01$ \\
620 & $0.0 \mathrm{E}+00$ & $2.1 \mathrm{E}+00$ & $2.1 \mathrm{E}-01$ \\
750 & $0.0 \mathrm{E}+00$ & $2.1 \mathrm{E}+00$ & $2.1 \mathrm{E}-01$ \\
1000 & $0.0 \mathrm{E}+00$ & $2.1 \mathrm{E}+00$ & $2.1 \mathrm{E}-01$ \\
1015 & $0.0 \mathrm{E}+00$ & $2.1 \mathrm{E}+00$ & $2.1 \mathrm{E}-01$ \\
1025 & $0.0 \mathrm{E}+00$ & $6.9 \mathrm{E}-01$ & $6.9 \mathrm{E}-02$ \\
1060 & $0.0 \mathrm{E}+00$ & $0.0 \mathrm{E}+00$ & $0.0 \mathrm{E}+00$ \\
1080 & $0.0 \mathrm{E}+00$ & $0.0 \mathrm{E}+00$ & $0.0 \mathrm{E}+00$ \\
1120 & $0.0 \mathrm{E}+00$ & $0.0 \mathrm{E}+00$ & $0.0 \mathrm{E}+00$ \\
1175 & $0.0 \mathrm{E}+00$ & $0.0 \mathrm{E}+00$ & $0.0 \mathrm{E}+00$ \\
1575 & $0.0 \mathrm{E}+00$ & $0.0 \mathrm{E}+00$ & $0.0 \mathrm{E}+00$ \\
1750 & $0.0 \mathrm{E}+00$ & $0.0 \mathrm{E}+00$ & $0.0 \mathrm{E}+00$ \\
2175 & $0.0 \mathrm{E}+00$ & $0.0 \mathrm{E}+00$ & $0.0 \mathrm{E}+00$ \\
4000 & $0.0 \mathrm{E}+00$ & $0.0 \mathrm{E}+00$ & $0.0 \mathrm{E}+00$ \\
6000 & $1.2 \mathrm{E}-03$ & $0.0 \mathrm{E}+00$ & $0.0 \mathrm{E}+00$ \\
8000 & $7.2 \mathrm{E}-03$ & $0.0 \mathrm{E}+00$ & $0.0 \mathrm{E}+00$ \\
10000 & $7.2 \mathrm{E}-03$ & $0.0 \mathrm{E}+00$ & $0.0 \mathrm{E}+00$ \\
& & & \\
& & &
\end{tabular}


GAVRM Cancer Risk from Lettuce Ingestion in Zone A Due to Groundwater Release

\begin{tabular}{rrrr}
\hline Time (Yr) & As & $\mathrm{Cr}$ & $\mathrm{Ni}$ \\
\hline 15 & $0.0 \mathrm{E}+00$ & $0.0 \mathrm{E}+00$ & $0.0 \mathrm{E}+00$ \\
20 & $0.0 \mathrm{E}+00$ & $0.0 \mathrm{E}+00$ & $0.0 \mathrm{E}+00$ \\
25 & $0.0 \mathrm{E}+00$ & $0.0 \mathrm{E}+00$ & $0.0 \mathrm{E}+00$ \\
30 & $0.0 \mathrm{E}+00$ & $0.0 \mathrm{E}+00$ & $0.0 \mathrm{E}+00$ \\
60 & $0.0 \mathrm{E}+00$ & $0.0 \mathrm{E}+00$ & $0.0 \mathrm{E}+00$ \\
62 & $0.0 \mathrm{E}+00$ & $0.0 \mathrm{E}+00$ & $0.0 \mathrm{E}+00$ \\
80 & $0.0 \mathrm{E}+00$ & $0.0 \mathrm{E}+00$ & $0.0 \mathrm{E}+00$ \\
100 & $0.0 \mathrm{E}+00$ & $0.0 \mathrm{E}+00$ & $0.0 \mathrm{E}+00$ \\
125 & $0.0 \mathrm{E}+00$ & $0.0 \mathrm{E}+00$ & $0.0 \mathrm{E}+00$ \\
575 & $0.0 \mathrm{E}+00$ & $0.0 \mathrm{E}+00$ & $0.0 \mathrm{E}+00$ \\
590 & $0.0 \mathrm{E}+00$ & $0.0 \mathrm{E}+00$ & $0.0 \mathrm{E}+00$ \\
620 & $0.0 \mathrm{E}+00$ & $0.0 \mathrm{E}+00$ & $0.0 \mathrm{E}+00$ \\
750 & $0.0 \mathrm{E}+00$ & $0.0 \mathrm{E}+00$ & $0.0 \mathrm{E}+00$ \\
1000 & $0.0 \mathrm{E}+00$ & $0.0 \mathrm{E}+00$ & $0.0 \mathrm{E}+00$ \\
1015 & $0.0 \mathrm{E}+00$ & $0.0 \mathrm{E}+00$ & $0.0 \mathrm{E}+00$ \\
1025 & $0.0 \mathrm{E}+00$ & $0.0 \mathrm{E}+00$ & $0.0 \mathrm{E}+00$ \\
1060 & $0.0 \mathrm{E}+00$ & $0.0 \mathrm{E}+00$ & $0.0 \mathrm{E}+00$ \\
1080 & $0.0 \mathrm{E}+00$ & $0.0 \mathrm{E}+00$ & $0.0 \mathrm{E}+00$ \\
1120 & $0.0 \mathrm{E}+00$ & $0.0 \mathrm{E}+00$ & $0.0 \mathrm{E}+00$ \\
1175 & $0.0 \mathrm{E}+00$ & $0.0 \mathrm{E}+00$ & $0.0 \mathrm{E}+00$ \\
1575 & $0.0 \mathrm{E}+00$ & $0.0 \mathrm{E}+00$ & $0.0 \mathrm{E}+00$ \\
1750 & $0.0 \mathrm{E}+00$ & $0.0 \mathrm{E}+00$ & $0.0 \mathrm{E}+00$ \\
2175 & $0.0 \mathrm{E}+00$ & $0.0 \mathrm{E}+00$ & $0.0 \mathrm{E}+00$ \\
4000 & $0.0 \mathrm{E}+00$ & $0.0 \mathrm{E}+00$ & $0.0 \mathrm{E}+00$ \\
6000 & $1.7 \mathrm{E}-04$ & $0.0 \mathrm{E}+00$ & $0.0 \mathrm{E}+00$ \\
8000 & $1.1 \mathrm{E}-03$ & $0.0 \mathrm{E}+00$ & $0.0 \mathrm{E}+00$ \\
10000 & $1.1 \mathrm{E}-03$ & $0.0 \mathrm{E}+00$ & $0.0 \mathrm{E}+00$ \\
& & & \\
\hline & & &
\end{tabular}

GBVRM Cancer Risk from Lettuce Ingestion in Zone $B$ Due to Groundwater Release

\begin{tabular}{rrrr}
\hline Time (Yr) & As & $\mathrm{Cr}$ & $\mathrm{Ni}$ \\
\hline 15 & $0.0 \mathrm{E}+00$ & $0.0 \mathrm{E}+00$ & $0.0 \mathrm{E}+00$ \\
20 & $0.0 \mathrm{E}+00$ & $0.0 \mathrm{E}+00$ & $0.0 \mathrm{E}+00$ \\
25 & $0.0 \mathrm{E}+00$ & $0.0 \mathrm{E}+00$ & $0.0 \mathrm{E}+00$ \\
30 & $0.0 \mathrm{E}+00$ & $0.0 \mathrm{E}+00$ & $0.0 \mathrm{E}+00$ \\
60 & $0.0 \mathrm{E}+00$ & $0.0 \mathrm{E}+00$ & $0.0 \mathrm{E}+00$ \\
62 & $0.0 \mathrm{E}+00$ & $0.0 \mathrm{E}+00$ & $0.0 \mathrm{E}+00$ \\
80 & $0.0 \mathrm{E}+00$ & $0.0 \mathrm{E}+00$ & $0.0 \mathrm{E}+00$ \\
100 & $0.0 \mathrm{E}+00$ & $0.0 \mathrm{E}+00$ & $0.0 \mathrm{E}+00$ \\
125 & $0.0 \mathrm{E}+00$ & $0.0 \mathrm{E}+00$ & $0.0 \mathrm{E}+00$ \\
575 & $0.0 \mathrm{E}+00$ & $0.0 \mathrm{E}+00$ & $0.0 \mathrm{E}+00$ \\
590 & $0.0 \mathrm{E}+00$ & $0.0 \mathrm{E}+00$ & $0.0 \mathrm{E}+00$ \\
620 & $0.0 \mathrm{E}+00$ & $0.0 \mathrm{E}+00$ & $0.0 \mathrm{E}+00$ \\
750 & $0.0 \mathrm{E}+00$ & $0.0 \mathrm{E}+00$ & $0.0 \mathrm{E}+00$ \\
1000 & $0.0 \mathrm{E}+00$ & $0.0 \mathrm{E}+00$ & $0.0 \mathrm{E}+00$ \\
1015 & $0.0 \mathrm{E}+00$ & $0.0 \mathrm{E}+00$ & $0.0 \mathrm{E}+00$ \\
1025 & $0.0 \mathrm{E}+00$ & $0.0 \mathrm{E}+00$ & $0.0 \mathrm{E}+00$ \\
1060 & $0.0 \mathrm{E}+00$ & $0.0 \mathrm{E}+00$ & $0.0 \mathrm{E}+00$ \\
1080 & $0.0 \mathrm{E}+00$ & $0.0 \mathrm{E}+00$ & $0.0 \mathrm{E}+00$ \\
1120 & $0.0 \mathrm{E}+00$ & $0.0 \mathrm{E}+00$ & $0.0 \mathrm{E}+00$ \\
1175 & $0.0 \mathrm{E}+00$ & $0.0 \mathrm{E}+00$ & $0.0 \mathrm{E}+00$ \\
1575 & $0.0 \mathrm{E}+00$ & $0.0 \mathrm{E}+00$ & $0.0 \mathrm{E}+00$ \\
1750 & $0.0 \mathrm{E}+00$ & $0.0 \mathrm{E}+00$ & $0.0 \mathrm{E}+00$ \\
2175 & $0.0 \mathrm{E}+00$ & $0.0 \mathrm{E}+00$ & $0.0 \mathrm{E}+00$ \\
4000 & $0.0 \mathrm{E}+00$ & $0.0 \mathrm{E}+00$ & $0.0 \mathrm{E}+00$ \\
6000 & $2.1 \mathrm{E}-05$ & $0.0 \mathrm{E}+00$ & $0.0 \mathrm{E}+00$ \\
8000 & $1.3 \mathrm{E}-04$ & $0.0 \mathrm{E}+00$ & $0.0 \mathrm{E}+00$ \\
10000 & $1.3 \mathrm{E}-04$ & $0.0 \mathrm{E}+00$ & $0.0 \mathrm{E}+00$
\end{tabular}


AAPCU Concentration in Pasture (Bq $\mathrm{kg}^{-1}$ ) in Zone $A$ Due to Atmospheric Release

\begin{tabular}{rrcccc}
\hline Time (yr) & $\mathrm{U}-238 \& 4$ & $\mathrm{Th}-230$ & Ra-226 & Pb-210 & Po-210 \\
\hline 1 & $1.5 \mathrm{E}-02$ & $1.2 \mathrm{E}-01$ & $1.2 \mathrm{E}-01$ & $1.2 \mathrm{E}-01$ & $1.2 \mathrm{E}-01$ \\
20 & $1.5 \mathrm{E}-02$ & $1.2 \mathrm{E}-01$ & $1.4 \mathrm{E}-01$ & $1.2 \mathrm{E}-01$ & $1.2 \mathrm{E}-01$ \\
25 & $1.5 \mathrm{E}-02$ & $1.2 \mathrm{E}-01$ & $1.5 \mathrm{E}-01$ & $1.2 \mathrm{E}-01$ & $1.2 \mathrm{E}-01$ \\
30 & $1.5 \mathrm{E}-02$ & $1.2 \mathrm{E}-01$ & $1.5 \mathrm{E}-01$ & $1.2 \mathrm{E}-01$ & $1.2 \mathrm{E}-01$ \\
62 & $1.6 \mathrm{E}-02$ & $1.2 \mathrm{E}-01$ & $1.8 \mathrm{E}-01$ & $1.2 \mathrm{E}-01$ & $1.3 \mathrm{E}-01$ \\
125 & $1.6 \mathrm{E}-02$ & $1.2 \mathrm{E}-01$ & $2.2 \mathrm{E}-01$ & $1.2 \mathrm{E}-01$ & $1.3 \mathrm{E}-01$ \\
575 & $1.7 \mathrm{E}-02$ & $1.2 \mathrm{E}-01$ & $2.8 \mathrm{E}-01$ & $1.3 \mathrm{E}-01$ & $1.4 \mathrm{E}-01$ \\
750 & $1.7 \mathrm{E}-02$ & $1.2 \mathrm{E}-01$ & $2.9 \mathrm{E}-01$ & $1.3 \mathrm{E}-01$ & $1.4 \mathrm{E}-01$ \\
1000 & $1.7 \mathrm{E}-02$ & $1.2 \mathrm{E}-01$ & $2.9 \mathrm{E}-01$ & $1.3 \mathrm{E}-01$ & $1.4 \mathrm{E}-01$ \\
1025 & $1.4 \mathrm{E}-03$ & $1.1 \mathrm{E}-03$ & $1.4 \mathrm{E}-01$ & $5.6 \mathrm{E}-03$ & $1.4 \mathrm{E}-02$ \\
1050 & $1.2 \mathrm{E}-03$ & $9.4 \mathrm{E}-04$ & $1.2 \mathrm{E}-01$ & $4.7 \mathrm{E}-03$ & $1.2 \mathrm{E}-02$ \\
1100 & $8.2 \mathrm{E}-04$ & $6.6 \mathrm{E}-04$ & $8.3 \mathrm{E}-02$ & $3.3 \mathrm{E}-03$ & $8.3 \mathrm{E}-03$ \\
1200 & $4.1 \mathrm{E}-04$ & $3.3 \mathrm{E}-04$ & $4.1 \mathrm{E}-02$ & $1.7 \mathrm{E}-03$ & $4.1 \mathrm{E}-03$ \\
1400 & $1.0 \mathrm{E}-04$ & $8.3 \mathrm{E}-05$ & $1.0 \mathrm{E}-02$ & $4.1 \mathrm{E}-04$ & $1.0 \mathrm{E}-03$ \\
1600 & $2.6 \mathrm{E}-05$ & $2.1 \mathrm{E}-05$ & $2.6 \mathrm{E}-03$ & $1.0 \mathrm{E}-04$ & $2.6 \mathrm{E}-04$ \\
2000 & $1.6 \mathrm{E}-06$ & $1.3 \mathrm{E}-06$ & $1.6 \mathrm{E}-04$ & $6.5 \mathrm{E}-06$ & $1.6 \mathrm{E}-05$ \\
2500 & $5.0 \mathrm{E}-08$ & $4.0 \mathrm{E}-08$ & $5.0 \mathrm{E}-06$ & $2.0 \mathrm{E}-07$ & $5.0 \mathrm{E}-07$ \\
3000 & $1.6 \mathrm{E}-09$ & $1.2 \mathrm{E}-09$ & $1.6 \mathrm{E}-07$ & $6.3 \mathrm{E}-09$ & $1.6 \mathrm{E}-08$ \\
& & & & & \\
\hline
\end{tabular}

ABPCU Concentration in Pasture (Bq $\mathrm{kg}^{-1}$ ) in Zone $B$ Due to Atmospheric Release

\begin{tabular}{rlllll}
\hline Time $(Y r)$ & $\mathrm{U}-238 \& 4$ & $\mathrm{Th}-230$ & $\mathrm{Ra}-226$ & $\mathrm{~Pb}-210$ & PO-210 \\
\hline 1 & $2.7 \mathrm{E}-03$ & $2.2 \mathrm{E}-02$ & $2.2 \mathrm{E}-02$ & $2.2 \mathrm{E}-02$ & $2.2 \mathrm{E}-02$ \\
20 & $2.8 \mathrm{E}-03$ & $2.2 \mathrm{E}-02$ & $2.6 \mathrm{E}-02$ & $2.2 \mathrm{E}-02$ & $2.2 \mathrm{E}-02$ \\
25 & $2.8 \mathrm{E}-03$ & $2.2 \mathrm{E}-02$ & $2.7 \mathrm{E}-02$ & $2.2 \mathrm{E}-02$ & $2.2 \mathrm{E}-02$ \\
30 & $2.8 \mathrm{E}-03$ & $2.2 \mathrm{E}-02$ & $2.8 \mathrm{E}-02$ & $2.2 \mathrm{E}-02$ & $2.2 \mathrm{E}-02$ \\
62 & $2.8 \mathrm{E}-03$ & $2.2 \mathrm{E}-02$ & $3.2 \mathrm{E}-02$ & $2.2 \mathrm{E}-02$ & $2.3 \mathrm{E}-02$ \\
125 & $2.9 \mathrm{E}-03$ & $2.2 \mathrm{E}-02$ & $3.9 \mathrm{E}-02$ & $2.3 \mathrm{E}-02$ & $2.4 \mathrm{E}-02$ \\
575 & $3.0 \mathrm{E}-03$ & $2.2 \mathrm{E}-02$ & $5.1 \mathrm{E}-02$ & $2.3 \mathrm{E}-02$ & $2.5 \mathrm{E}-02$ \\
750 & $3.0 \mathrm{E}-03$ & $2.2 \mathrm{E}-02$ & $5.2 \mathrm{E}-02$ & $2.3 \mathrm{E}-02$ & $2.5 \mathrm{E}-02$ \\
1000 & $3.0 \mathrm{E}-03$ & $2.2 \mathrm{E}-02$ & $5.2 \mathrm{E}-02$ & $2.3 \mathrm{E}-02$ & $2.5 \mathrm{E}-02$ \\
1025 & $2.5 \mathrm{E}-04$ & $2.0 \mathrm{E}-04$ & $2.5 \mathrm{E}-02$ & $1.0 \mathrm{E}-03$ & $2.5 \mathrm{E}-03$ \\
1050 & $2.1 \mathrm{E}-04$ & $1.7 \mathrm{E}-04$ & $2.1 \mathrm{E}-02$ & $8.6 \mathrm{E}-04$ & $2.1 \mathrm{E}-03$ \\
1100 & $1.5 \mathrm{E}-04$ & $1.2 \mathrm{E}-04$ & $1.5 \mathrm{E}-02$ & $6.0 \mathrm{E}-04$ & $1.5 \mathrm{E}-03$ \\
1200 & $7.5 \mathrm{E}-05$ & $6.0 \mathrm{E}-05$ & $7.5 \mathrm{E}-03$ & $3.0 \mathrm{E}-04$ & $7.5 \mathrm{E}-04$ \\
1400 & $1.9 \mathrm{E}-05$ & $1.5 \mathrm{E}-05$ & $1.9 \mathrm{E}-03$ & $7.6 \mathrm{E}-05$ & $1.9 \mathrm{E}-04$ \\
1600 & $4.7 \mathrm{E}-06$ & $3.7 \mathrm{E}-06$ & $4.7 \mathrm{E}-04$ & $1.9 \mathrm{E}-05$ & $4.7 \mathrm{E}-05$ \\
2000 & $2.9 \mathrm{E}-07$ & $2.3 \mathrm{E}-07$ & $2.9 \mathrm{E}-05$ & $1.2 \mathrm{E}-06$ & $2.9 \mathrm{E}-06$ \\
2500 & $9.2 \mathrm{E}-09$ & $7.3 \mathrm{E}-09$ & $9.2 \mathrm{E}-07$ & $3.7 \mathrm{E}-08$ & $9.2 \mathrm{E}-08$ \\
3000 & $2.9 \mathrm{E}-10$ & $2.3 \mathrm{E}-10$ & $2.9 \mathrm{E}-08$ & $1.1 \mathrm{E}-09$ & $2.9 \mathrm{E}-09$ \\
\end{tabular}


AAPCM Concentration in Pasture (mg $\mathrm{kg}^{-1}$ ) in Zone A Due to Atmospheric Release

\begin{tabular}{rrrr}
\hline Time $(y r)$ & As & Cr & $N i$ \\
\hline 1 & $8.6 \mathrm{E}-04$ & $8.6 \mathrm{E}-05$ & $8.6 \mathrm{E}-06$ \\
20 & $8.7 \mathrm{E}-04$ & $8.9 \mathrm{E}-05$ & $8.8 \mathrm{E}-06$ \\
25 & $8.8 \mathrm{E}-04$ & $9.0 \mathrm{E}-05$ & $8.8 \mathrm{E}-06$ \\
30 & $8.8 \mathrm{E}-04$ & $9.1 \mathrm{E}-05$ & $8.8 \mathrm{E}-06$ \\
62 & $9.0 \mathrm{E}-04$ & $9.5 \mathrm{E}-05$ & $9.0 \mathrm{E}-06$ \\
125 & $9.2 \mathrm{E}-04$ & $1.0 \mathrm{E}-04$ & $9.3 \mathrm{E}-06$ \\
575 & $9.6 \mathrm{E}-04$ & $1.1 \mathrm{E}-04$ & $9.8 \mathrm{E}-06$ \\
750 & $9.6 \mathrm{E}-04$ & $1.1 \mathrm{E}-04$ & $9.8 \mathrm{E}-06$ \\
1000 & $9.6 \mathrm{E}-04$ & $1.1 \mathrm{E}-04$ & $9.8 \mathrm{E}-06$ \\
1025 & $8.0 \mathrm{E}-05$ & $2.0 \mathrm{E}-05$ & $1.0 \mathrm{E}-06$ \\
1050 & $6.7 \mathrm{E}-05$ & $1.7 \mathrm{E}-05$ & $8.4 \mathrm{E}-07$ \\
1100 & $4.8 \mathrm{E}-05$ & $1.2 \mathrm{E}-05$ & $6.0 \mathrm{E}-07$ \\
1200 & $2.4 \mathrm{E}-05$ & $6.0 \mathrm{E}-06$ & $3.0 \mathrm{E}-07$ \\
1400 & $6.0 \mathrm{E}-06$ & $1.5 \mathrm{E}-06$ & $7.4 \mathrm{E}-08$ \\
1600 & $1.5 \mathrm{E}-06$ & $3.7 \mathrm{E}-07$ & $1.9 \mathrm{E}-08$ \\
2000 & $9.3 \mathrm{E}-08$ & $2.3 \mathrm{E}-08$ & $1.2 \mathrm{E}-09$ \\
2500 & $2.9 \mathrm{E}-09$ & $7.3 \mathrm{E}-10$ & $3.6 \mathrm{E}-11$ \\
3000 & $9.1 \mathrm{E}-11$ & $2.3 \mathrm{E}-11$ & $1.1 \mathrm{E}-12$ \\
& & &
\end{tabular}

ABPCM Concentration in Pasture (mg $\mathrm{kg}^{-1}$ ) in Zone $B$ Due to Atmospheric Release

\begin{tabular}{rrrr}
\hline Time $(\mathrm{Y} x)$ & As & $\mathrm{Cr}$ & $\mathrm{Ni}$ \\
\hline 1 & $1.6 \mathrm{E}-04$ & $1.6 \mathrm{E}-05$ & $1.6 \mathrm{E}-06$ \\
20 & $1.6 \mathrm{E}-04$ & $1.6 \mathrm{E}-05$ & $1.6 \mathrm{E}-06$ \\
25 & $1.6 \mathrm{E}-04$ & $1.6 \mathrm{E}-05$ & $1.6 \mathrm{E}-06$ \\
30 & $1.6 \mathrm{E}-04$ & $1.6 \mathrm{E}-05$ & $1.6 \mathrm{E}-06$ \\
62 & $1.6 \mathrm{E}-04$ & $1.7 \mathrm{E}-05$ & $1.6 \mathrm{E}-06$ \\
125 & $1.7 \mathrm{E}-04$ & $1.8 \mathrm{E}-05$ & $1.7 \mathrm{E}-06$ \\
575 & $1.7 \mathrm{E}-04$ & $2.0 \mathrm{E}-05$ & $1.8 \mathrm{E}-06$ \\
750 & $1.7 \mathrm{E}-04$ & $2.0 \mathrm{E}-05$ & $1.8 \mathrm{E}-06$ \\
1000 & $1.7 \mathrm{E}-04$ & $2.0 \mathrm{E}-05$ & $1.8 \mathrm{E}-06$ \\
1025 & $1.4 \mathrm{E}-05$ & $3.6 \mathrm{E}-06$ & $1.8 \mathrm{E}-07$ \\
1050 & $1.2 \mathrm{E}-05$ & $3.0 \mathrm{E}-06$ & $1.5 \mathrm{E}-07$ \\
1100 & $8.6 \mathrm{E}-06$ & $2.2 \mathrm{E}-06$ & $1.1 \mathrm{E}-07$ \\
1200 & $4.3 \mathrm{E}-06$ & $1.1 \mathrm{E}-06$ & $5.4 \mathrm{E}-08$ \\
1400 & $1.1 \mathrm{E}-06$ & $2.7 \mathrm{E}-07$ & $1.3 \mathrm{E}-08$ \\
1600 & $2.7 \mathrm{E}-07$ & $6.7 \mathrm{E}-08$ & $3.4 \mathrm{E}-09$ \\
2000 & $1.7 \mathrm{E}-08$ & $4.2 \mathrm{E}-09$ & $2.1 \mathrm{E}-10$ \\
2500 & $5.2 \mathrm{E}-10$ & $1.3 \mathrm{E}-10$ & $6.6 \mathrm{E}-12$ \\
3000 & $1.6 \mathrm{E}-11$ & $4.1 \mathrm{E}-12$ & $2.1 \mathrm{E}-13$
\end{tabular}

AASCU Concentration in Soil $\left(\mathrm{Bq} \mathrm{m}^{-3}\right)$ in zone $A$ Due to Atmospheric Release

\begin{tabular}{|c|c|c|c|c|c|c|}
\hline Time & (YY) & $U-238 \& 4$ & $\mathrm{Th}-230$ & $\mathrm{Ra}-226$ & $\mathrm{~Pb}-210$ & Po- 210 \\
\hline & 1 & 4. $0 E+00$ & 3. $2 \mathrm{E}+01$ & 3. $2 \mathrm{E}+01$ & $3.2 E+01$ & $3.2 \mathrm{E}+01$ \\
\hline & 20 & $7.5 E+01$ & $6.0 E+02$ & $6.0 E+02$ & $6.0 E+02$ & $6.0 \mathrm{E}+02$ \\
\hline & 25 & $9.2 \mathrm{E}+01$ & $7.4 \mathrm{E}+02$ & $7.4 \mathrm{E}+02$ & $7.4 \mathrm{E}+02$ & $7.4 E+02$ \\
\hline & 30 & 1. $1 E+02$ & $8.7 E+02$ & $8.7 \mathrm{E}+02$ & $8.7 E+02$ & $8.7 E+02$ \\
\hline & 62 & $2.0 E+02$ & 1. $6 E+03$ & 1. $6 E+03$ & 1. $6 \mathrm{E}+03$ & 1. $6 E+03$ \\
\hline & 125 & $3.4 \mathrm{E}+02$ & $2.7 E+03$ & $2.7 \mathrm{E}+03$ & $2.7 \mathrm{E}+03$ & $2.7 E+03$ \\
\hline & 575 & $5.7 E+02$ & 4. $6 E+03$ & $4.6 \mathrm{E}+03$ & $4.6 \mathrm{E}+03$ & $4.6 \mathrm{E}+03$ \\
\hline & 750 & $5.8 E+02$ & $4.6 E+03$ & $4.6 E+03$ & $4.6 \mathrm{E}+03$ & $4.6 \mathrm{E}+03$ \\
\hline & 1000 & $5.8 E+02$ & $4.6 E+03$ & $4.7 E+03$ & $4.7 E+03$ & $4.7 E+03$ \\
\hline & 1025 & $4.9 E+02$ & $3.9 E+03$ & $3.9 E+03$ & $3.9 E+03$ & $3.9 E+03$ \\
\hline & 1050 & $4.1 E+02$ & 3. $3 E+03$ & $3.3 E+03$ & $3.3 E+03$ & 3. $3 E+03$ \\
\hline & 1100 & $2.9 E+02$ & $2.3 E+03$ & $2.3 E+03$ & $2.3 E+03$ & $2.3 E+03$ \\
\hline & 1200 & 1. $4 E+02$ & 1. $2 E+03$ & 1. $2 \mathrm{E}+03$ & 1. $2 \mathrm{E}+03$ & 1. $2 \mathrm{E}+03$ \\
\hline & 1400 & $3.6 \mathrm{E}+01$ & $2.9 E+02$ & 2. $9 E+02$ & $2.9 \mathrm{E}+02$ & $2.9 \mathrm{E}+02$ \\
\hline & 1600 & $9.0 \mathrm{E}+00$ & $7.2 \mathrm{E}+01$ & 7. $3 E+01$ & $7.3 E+01$ & $7.3 E+01$ \\
\hline & 2000 & $5.6 \mathrm{E}-01$ & $4.5 E+00$ & $4.5 E+00$ & $4.5 E+00$ & 4. $5 E+00$ \\
\hline & 2500 & 1. $8 E-02$ & 1. $4 \mathrm{E}-01$ & 1. $4 \mathrm{E}-01$ & 1. $4 \mathrm{E}-01$ & 1. $4 \mathrm{E}-01$ \\
\hline & 3000 & $5.5 \mathrm{E}-04$ & $4.4 \mathrm{E}-03$ & $4.4 \mathrm{E}-03$ & $4.4 \mathrm{E}-03$ & $4.4 \mathrm{E}-03$ \\
\hline
\end{tabular}


AASDU External (Ground) Irradiation in Zone A Due to Atmospheric Release (Sv $\mathrm{yr}^{-1}$ )

\begin{tabular}{rllllll}
\hline Time $(\mathrm{yr})$ & $\mathrm{U}-238 \& 4$ & Th-230 & Ra-226 & Pb-210 & Po-210 & Chain \\
\hline 1 & $3.4 \mathrm{E}-11$ & $8.9 \mathrm{E}-12$ & $6.3 \mathrm{E}-08$ & $2.0 \mathrm{E}-11$ & $2.5 \mathrm{E}-13$ & $6.3 \mathrm{E}-08$ \\
20 & $6.4 \mathrm{E}-10$ & $1.7 \mathrm{E}-10$ & $1.2 \mathrm{E}-06$ & $3.7 \mathrm{E}-10$ & $4.7 \mathrm{E}-12$ & $1.2 \mathrm{E}-06$ \\
35 & $7.8 \mathrm{E}-10$ & $2.1 \mathrm{E}-10$ & $1.4 \mathrm{E}-06$ & $4.6 \mathrm{E}-10$ & $5.8 \mathrm{E}-12$ & $1.4 \mathrm{E}-06$ \\
30 & $9.2 \mathrm{E}-10$ & $2.4 \mathrm{E}-10$ & $1.7 \mathrm{E}-06$ & $5.4 \mathrm{E}-10$ & $6.9 \mathrm{E}-12$ & $1.7 \mathrm{E}-06$ \\
62 & $1.7 \mathrm{E}-09$ & $4.5 \mathrm{E}-10$ & $3.2 \mathrm{E}-06$ & $1.0 \mathrm{E}-09$ & $1.3 \mathrm{E}-11$ & $3.2 \mathrm{E}-06$ \\
125 & $2.9 \mathrm{E}-09$ & $7.5 \mathrm{E}-10$ & $5.3 \mathrm{E}-06$ & $1.7 \mathrm{E}-09$ & $2.1 \mathrm{E}-11$ & $5.3 \mathrm{E}-06$ \\
575 & $4.8 \mathrm{E}-09$ & $1.3 \mathrm{E}-09$ & $8.9 \mathrm{E}-06$ & $2.8 \mathrm{E}-09$ & $3.6 \mathrm{E}-11$ & $8.9 \mathrm{E}-06$ \\
750 & $4.9 \mathrm{E}-09$ & $1.3 \mathrm{E}-09$ & $9.0 \mathrm{E}-06$ & $2.9 \mathrm{E}-09$ & $3.6 \mathrm{E}-11$ & $9.0 \mathrm{E}-06$ \\
1000 & $4.9 \mathrm{E}-09$ & $1.3 \mathrm{E}-09$ & $9.1 \mathrm{E}-06$ & $2.9 \mathrm{E}-09$ & $3.6 \mathrm{E}-11$ & $9.1 \mathrm{E}-06$ \\
1025 & $4.1 \mathrm{E}-09$ & $1.1 \mathrm{E}-09$ & $7.6 \mathrm{E}-06$ & $2.4 \mathrm{E}-09$ & $3.1 \mathrm{E}-11$ & $7.6 \mathrm{E}-06$ \\
1050 & $3.5 \mathrm{E}-09$ & $9.1 \mathrm{E}-10$ & $6.4 \mathrm{E}-06$ & $2.0 \mathrm{E}-09$ & $2.6 \mathrm{E}-11$ & $6.4 \mathrm{E}-06$ \\
1100 & $2.5 \mathrm{E}-09$ & $6.5 \mathrm{E}-10$ & $4.5 \mathrm{E}-06$ & $1.4 \mathrm{E}-09$ & $1.8 \mathrm{E}-11$ & $4.5 \mathrm{E}-06$ \\
1200 & $1.2 \mathrm{E}-09$ & $3.2 \mathrm{E}-10$ & $2.3 \mathrm{E}-06$ & $7.2 \mathrm{E}-10$ & $9.1 \mathrm{E}-12$ & $2.3 \mathrm{E}-06$ \\
1400 & $3.1 \mathrm{E}-10$ & $8.0 \mathrm{E}-11$ & $5.7 \mathrm{E}-07$ & $1.8 \mathrm{E}-10$ & $2.3 \mathrm{E}-12$ & $5.7 \mathrm{E}-07$ \\
1600 & $7.7 \mathrm{E}-11$ & $2.0 \mathrm{E}-11$ & $1.4 \mathrm{E}-07$ & $4.5 \mathrm{E}-11$ & $5.7 \mathrm{E}-13$ & $1.4 \mathrm{E}-07$ \\
2000 & $4.8 \mathrm{E}-12$ & $1.3 \mathrm{E}-12$ & $8.8 \mathrm{E}-09$ & $2.8 \mathrm{E}-12$ & $3.6 \mathrm{E}-14$ & $8.8 \mathrm{E}-09$ \\
2500 & $1.5 \mathrm{E}-13$ & $3.9 \mathrm{E}-14$ & $2.8 \mathrm{E}-10$ & $8.7 \mathrm{E}-14$ & $1.1 \mathrm{E}-15$ & $2.8 \mathrm{E}-10$ \\
3000 & $4.7 \mathrm{E}-15$ & $1.2 \mathrm{E}-15$ & $8.6 \mathrm{E}-12$ & $2.7 \mathrm{E}-15$ & $3.5 \mathrm{E}-17$ & $8.6 \mathrm{E}-12$ \\
\hline
\end{tabular}

AASRU Cancer Risk from External (Ground) Irradiation in Zone A Due to Atmospheric Release

\begin{tabular}{rrrrrr}
\hline Time $(Y r)$ & $U-238 \& 4$ & $T h-230$ & Ra-226 & Pb-210 & Po-210 \\
\hline 1 & $6.4 E-11$ & $1.7 E-12$ & $1.6 E-07$ & $3.9 E-12$ & $8.2 \mathrm{E}-13$ \\
20 & $1.2 \mathrm{E}-09$ & $3.2 \mathrm{E}-11$ & $3.0 \mathrm{E}-06$ & $7.3 \mathrm{E}-11$ & $1.5 \mathrm{E}-11$ \\
30 & $1.5 \mathrm{E}-09$ & $3.9 \mathrm{E}-11$ & $3.7 \mathrm{E}-06$ & $8.9 \mathrm{E}-11$ & $1.9 \mathrm{E}-11$ \\
62 & $3.2 \mathrm{E}-09$ & $4.6 \mathrm{E}-11$ & $4.4 \mathrm{E}-06$ & $1.1 \mathrm{E}-10$ & $2.2 \mathrm{E}-11$ \\
125 & $5.4 \mathrm{E}-09$ & $1.4 \mathrm{E}-11$ & $8.1 \mathrm{E}-06$ & $2.0 \mathrm{E}-10$ & $4.1 \mathrm{E}-11$ \\
575 & $9.1 \mathrm{E}-09$ & $2.4 \mathrm{E}-10$ & $2.3 \mathrm{E}-05$ & $3.3 \mathrm{E}-10$ & $5.9 \mathrm{E}-11$ \\
750 & $9.2 \mathrm{E}-09$ & $2.4 \mathrm{E}-10$ & $2.3 \mathrm{E}-05$ & $5.6 \mathrm{E}-10$ & $1.2 \mathrm{E}-10$ \\
1000 & $9.3 \mathrm{E}-09$ & $2.4 \mathrm{E}-10$ & $2.3 \mathrm{E}-05$ & $5.6 \mathrm{E}-10$ & $1.2 \mathrm{E}-10$ \\
1025 & $7.8 \mathrm{E}-09$ & $2.0 \mathrm{E}-10$ & $2.0 \mathrm{E}-05$ & $4.7 \mathrm{E}-10$ & $9.9 \mathrm{E}-11$ \\
1050 & $6.6 \mathrm{E}-09$ & $1.7 \mathrm{E}-10$ & $1.6 \mathrm{E}-05$ & $4.0 \mathrm{E}-10$ & $8.4 \mathrm{E}-11$ \\
1100 & $4.6 \mathrm{E}-09$ & $1.2 \mathrm{E}-10$ & $1.2 \mathrm{E}-05$ & $2.8 \mathrm{E}-10$ & $5.9 \mathrm{E}-11$ \\
1200 & $2.3 \mathrm{E}-09$ & $6.1 \mathrm{E}-11$ & $5.8 \mathrm{E}-06$ & $1.4 \mathrm{E}-10$ & $3.0 \mathrm{E}-11$ \\
1400 & $5.8 \mathrm{E}-10$ & $1.5 \mathrm{E}-11$ & $1.5 \mathrm{E}-06$ & $3.5 \mathrm{E}-11$ & $7.4 \mathrm{E}-12$ \\
1600 & $1.5 \mathrm{E}-10$ & $3.8 \mathrm{E}-12$ & $3.6 \mathrm{E}-07$ & $8.8 \mathrm{E}-12$ & $1.8 \mathrm{E}-12$ \\
2000 & $9.1 \mathrm{E}-12$ & $2.4 \mathrm{E}-13$ & $2.3 \mathrm{E}-08$ & $5.5 \mathrm{E}-13$ & $1.2 \mathrm{E}-13$ \\
2500 & $2.8 \mathrm{E}-13$ & $7.3 \mathrm{E}-15$ & $7.1 \mathrm{E}-10$ & $1.7 \mathrm{E}-14$ & $3.6 \mathrm{E}-15$ \\
3000 & $8.9 \mathrm{E}-15$ & $2.3 \mathrm{E}-16$ & $2.2 \mathrm{E}-11$ & $5.3 \mathrm{E}-16$ & $1.1 \mathrm{E}-16$
\end{tabular}


ABSCU Concentration in Soil $\left(\mathrm{Bq} \mathrm{m}^{-3}\right)$ in Zone $B$ Due to Atmospheric Release

\begin{tabular}{|c|c|c|c|c|c|}
\hline Time & $U-238 \& 4$ & $\mathrm{Th}-230$ & $R a-226$ & $\mathrm{~Pb}-210$ & Po-210 \\
\hline 1 & $7.2 \mathrm{E}-01$ & $5.8 E+00$ & $5.8 E+00$ & $5.8 E+00$ & $5.8 \mathrm{E}+00$ \\
\hline 20 & 1. $4 \mathrm{E}+01$ & 1. $1 E+02$ & 1.1E+02 & $1.1 E+02$ & $1.1 E+02$ \\
\hline 25 & 1. $7 \mathrm{E}+01$ & 1. $3 E+02$ & 1. $3 E+02$ & 1. $3 E+02$ & 1. $3 E \div 02$ \\
\hline 30 & $2.0 E+01$ & 1. $6 \mathrm{E}+02$ & 1. $6 \mathrm{E}+02$ & 1. $6 \mathrm{E}+02$ & 1. $6 \mathrm{E}+02$ \\
\hline 62 & $3.7 E+01$ & $2.9 E+02$ & $2.9 E+02$ & $2.9 E+02$ & $2.9 E+02$ \\
\hline 125 & $6.1 E+01$ & $4.9 E+02$ & $4.9 \mathrm{E}+02$ & $4.9 \mathrm{E}+02$ & $4.9 E+02$ \\
\hline 575 & 1. $0 E+02$ & $8.3 E+02$ & $8.3 E+02$ & $8.3 E+02$ & $8.3 E+02$ \\
\hline 750 & 1. $0 \mathrm{E}+02$ & $8.4 E+02$ & $8.4 \mathrm{E}+02$ & $8.4 E+02$ & $8.4 E+02$ \\
\hline 1000 & $1.0 E+02$ & $8.4 E+02$ & $8.4 E+02$ & $8.4 E+02$ & $8.4 E+02$ \\
\hline 1025 & $8.8 E+01$ & $7.1 E+02$ & $7.1 E+02$ & $7.1 \mathrm{E}+02$ & $7.1 E+02$ \\
\hline 1050 & $7.4 E+01$ & $6.0 E+02$ & $6.0 E+02$ & $6.0 E+02$ & $6.0 E+02$ \\
\hline 1100 & $5.2 \mathrm{E}+01$ & $4.2 E+02$ & $4.2 E+02$ & $4.2 E+02$ & $4.2 E+02$ \\
\hline 1200 & $2.6 E \div 01$ & $2.1 E+02$ & $2.1 E+02$ & $2.1 E+02$ & $2.1 E+02$ \\
\hline 1400 & $6.5 E+00$ & $5.2 E+01$ & 5. $3 E+01$ & $5.3 E+01$ & $5.3 E+01$ \\
\hline 1600 & 1. $6 E+00$ & 1. $3 E+01$ & 1. $3 E+01$ & 1. $3 E+01$ & 1. $3 \mathrm{E}+01$ \\
\hline 2000 & $1.0 E-01$ & $8.2 \mathrm{E}-01$ & $8.2 \mathrm{E}-01$ & $8.2 \mathrm{E}-01$ & $8.2 \mathrm{E}-01$ \\
\hline 2500 & $3.2 \mathrm{E}-03$ & $2.5 E-02$ & $2.6 \mathrm{E}-02$ & $2.6 \mathrm{E}-02$ & $2.6 E-02$ \\
\hline 3000 & $1.0 \mathrm{E}-04$ & $7.9 \mathrm{E}-04$ & $8.0 \mathrm{E}-04$ & 8. $0 \mathrm{E}-04$ & $8.0 E-04$ \\
\hline
\end{tabular}

ABSDU External (Ground) Irradiation in Zone B Due to Atmospheric Release (Sv $\mathrm{yr}^{-1}$ )

\begin{tabular}{rllllll}
\hline Time $(\mathrm{Yr})$ & $\mathrm{U}-238 \& 4$ & Th-230 & Ra-226 & Pb-210 & Po-210 & Chain \\
\hline 1 & $6.2 \mathrm{E}-12$ & $1.6 \mathrm{E}-12$ & $1.1 \mathrm{E}-08$ & $3.6 \mathrm{E}-12$ & $4.6 \mathrm{E}-14$ & $1.1 \mathrm{E}-08$ \\
20 & $1.2 \mathrm{E}-10$ & $3.0 \mathrm{E}-11$ & $2.1 \mathrm{E}-07$ & $6.8 \mathrm{E}-11$ & $8.6 \mathrm{E}-13$ & $2.1 \mathrm{E}-07$ \\
30 & $1.4 \mathrm{E}-10$ & $3.7 \mathrm{E}-11$ & $2.6 \mathrm{E}-07$ & $8.3 \mathrm{E}-11$ & $1.1 \mathrm{E}-12$ & $2.6 \mathrm{E}-07$ \\
62 & $3.7 \mathrm{E}-10$ & $4.4 \mathrm{E}-11$ & $3.1 \mathrm{E}-07$ & $9.8 \mathrm{E}-11$ & $1.2 \mathrm{E}-12$ & $3.1 \mathrm{E}-07$ \\
125 & $5.2 \mathrm{E}-10$ & $8.2 \mathrm{E}-11$ & $5.8 \mathrm{E}-07$ & $1.8 \mathrm{E}-10$ & $2.3 \mathrm{E}-12$ & $5.8 \mathrm{E}-07$ \\
575 & $8.8 \mathrm{E}-10$ & $2.3 \mathrm{E}-10$ & $9.5 \mathrm{E}-07$ & $3.0 \mathrm{E}-10$ & $3.8 \mathrm{E}-12$ & $9.6 \mathrm{E}-07$ \\
750 & $8.9 \mathrm{E}-10$ & $2.3 \mathrm{E}-10$ & $1.6 \mathrm{E}-06$ & $5.1 \mathrm{E}-10$ & $6.5 \mathrm{E}-12$ & $1.6 \mathrm{E}-06$ \\
1000 & $8.9 \mathrm{E}-10$ & $2.3 \mathrm{E}-10$ & $1.6 \mathrm{E}-06$ & $5.2 \mathrm{E}-10$ & $6.6 \mathrm{E}-12$ & $1.6 \mathrm{E}-06$ \\
1025 & $7.5 \mathrm{E}-10$ & $2.0 \mathrm{E}-10$ & $1.4 \mathrm{E}-06$ & $4.4 \mathrm{E}-10$ & $5.6 \mathrm{E}-12$ & $1.6 \mathrm{E}-06$ \\
1050 & $6.3 \mathrm{E}-10$ & $1.7 \mathrm{E}-10$ & $1.2 \mathrm{E}-06$ & $3.7 \mathrm{E}-10$ & $4.7 \mathrm{E}-12$ & $1.2 \mathrm{E}-06$ \\
1100 & $4.5 \mathrm{E}-10$ & $1.2 \mathrm{E}-10$ & $8.2 \mathrm{E}-07$ & $2.6 \mathrm{E}-10$ & $3.3 \mathrm{E}-12$ & $8.2 \mathrm{E}-07$ \\
1200 & $2.2 \mathrm{E}-10$ & $5.8 \mathrm{E}-11$ & $4.1 \mathrm{E}-07$ & $1.3 \mathrm{E}-10$ & $1.7 \mathrm{E}-12$ & $4.1 \mathrm{E}-07$ \\
1400 & $5.6 \mathrm{E}-11$ & $1.5 \mathrm{E}-11$ & $1.0 \mathrm{E}-07$ & $3.3 \mathrm{E}-11$ & $4.1 \mathrm{E}-13$ & $1.0 \mathrm{E}-07$ \\
1600 & $1.4 \mathrm{E}-11$ & $3.6 \mathrm{E}-12$ & $2.6 \mathrm{E}-08$ & $8.1 \mathrm{E}-12$ & $1.0 \mathrm{E}-13$ & $2.6 \mathrm{E}-08$ \\
2000 & $8.7 \mathrm{E}-13$ & $2.3 \mathrm{E}-13$ & $1.6 \mathrm{E}-09$ & $5.1 \mathrm{E}-13$ & $6.5 \mathrm{E}-15$ & $1.6 \mathrm{E}-09$ \\
2500 & $2.7 \mathrm{E}-14$ & $7.1 \mathrm{E}-15$ & $5.0 \mathrm{E}-11$ & $1.6 \mathrm{E}-14$ & $2.0 \mathrm{E}-16$ & $5.0 \mathrm{E}-11$ \\
3000 & $8.5 \mathrm{E}-16$ & $2.2 \mathrm{E}-16$ & $1.6 \mathrm{E}-12$ & $4.9 \mathrm{E}-16$ & $6.3 \mathrm{E}-18$ & $1.6 \mathrm{E}-12$ \\
\hline
\end{tabular}


ABSRU Cancer Risk from External (Ground) Irradiation in Zone $B$ Due to Atmospheric Release

\begin{tabular}{rccccc}
\hline Time $(\mathrm{Y} Y)$ & $\mathrm{U}-238 \& 4$ & Th-230 & Ra-226 & Pb-210 & Po-210 \\
& & & & & \\
20 & $1.2 \mathrm{E}-11$ & $3.1 \mathrm{E}-13$ & $2.9 \mathrm{E}-08$ & $7.1 \mathrm{E}-13$ & $1.5 \mathrm{E}-13$ \\
25 & $2.7 \mathrm{E}-10$ & $5.7 \mathrm{E}-12$ & $5.5 \mathrm{E}-07$ & $1.3 \mathrm{E}-11$ & $2.8 \mathrm{E}-12$ \\
30 & $3.2 \mathrm{E}-10$ & $8.3 \mathrm{E}-12$ & $6.7 \mathrm{E}-07$ & $1.6 \mathrm{E}-11$ & $3.4 \mathrm{E}-12$ \\
62 & $5.9 \mathrm{E}-10$ & $1.5 \mathrm{E}-11$ & $1.5 \mathrm{E}-07$ & $1.9 \mathrm{E}-11$ & $4.0 \mathrm{E}-12$ \\
125 & $9.8 \mathrm{E}-10$ & $2.6 \mathrm{E}-11$ & $2.5 \mathrm{E}-06$ & $5.9 \mathrm{E}-11$ & $7.5 \mathrm{E}-12$ \\
575 & $1.7 \mathrm{E}-09$ & $4.3 \mathrm{E}-11$ & $4.2 \mathrm{E}-06$ & $1.0 \mathrm{E}-10$ & $2.1 \mathrm{E}-11$ \\
750 & $1.7 \mathrm{E}-09$ & $4.4 \mathrm{E}-11$ & $4.2 \mathrm{E}-06$ & $1.0 \mathrm{E}-10$ & $2.1 \mathrm{E}-11$ \\
1000 & $1.7 \mathrm{E}-09$ & $4.4 \mathrm{E}-11$ & $4.2 \mathrm{E}-06$ & $1.0 \mathrm{E}-10$ & $2.1 \mathrm{E}-11$ \\
1025 & $1.4 \mathrm{E}-09$ & $3.7 \mathrm{E}-11$ & $3.6 \mathrm{E}-06$ & $8.6 \mathrm{E}-11$ & $1.8 \mathrm{E}-11$ \\
1050 & $1.2 \mathrm{E}-09$ & $3.1 \mathrm{E}-11$ & $3.0 \mathrm{E}-06$ & $7.2 \mathrm{E}-11$ & $1.5 \mathrm{E}-11$ \\
1100 & $8.4 \mathrm{E}-10$ & $2.2 \mathrm{E}-11$ & $2.1 \mathrm{E}-06$ & $5.1 \mathrm{E}-11$ & $1.1 \mathrm{E}-11$ \\
1200 & $4.2 \mathrm{E}-10$ & $1.1 \mathrm{E}-11$ & $1.1 \mathrm{E}-06$ & $2.5 \mathrm{E}-11$ & $5.4 \mathrm{E}-12$ \\
1400 & $1.1 \mathrm{E}-10$ & $2.7 \mathrm{E}-12$ & $2.6 \mathrm{E}-07$ & $6.4 \mathrm{E}-12$ & $1.3 \mathrm{E}-12$ \\
1600 & $2.6 \mathrm{E}-11$ & $6.9 \mathrm{E}-13$ & $6.6 \mathrm{E}-08$ & $1.6 \mathrm{E}-12$ & $3.3 \mathrm{E}-13$ \\
2000 & $1.6 \mathrm{E}-12$ & $4.3 \mathrm{E}-14$ & $4.1 \mathrm{E}-09$ & $9.9 \mathrm{E}-14$ & $2.1 \mathrm{E}-14$ \\
2500 & $5.1 \mathrm{E}-14$ & $1.3 \mathrm{E}-15$ & $1.3 \mathrm{E}-10$ & $3.1 \mathrm{E}-15$ & $6.5 \mathrm{E}-16$ \\
3000 & $1.6 \mathrm{E}-15$ & $4.1 \mathrm{E}-17$ & $4.0 \mathrm{E}-12$ & $9.7 \mathrm{E}-17$ & $2.0 \mathrm{E}-17$
\end{tabular}

AASCM Concentration in Soil ( $\mathrm{mg} \mathrm{m}^{-3}$ ) in Zone A Due to Atmospheric Release

\begin{tabular}{rrrr}
\hline Time $(Y r)$ & As & Cr & $N i$ \\
\hline 1 & $2.3 E-01$ & $2.3 E-02$ & $2.3 E-03$ \\
20 & $4.3 E+00$ & $4.3 E-01$ & $4.3 E-02$ \\
25 & $5.3 E+00$ & $5.3 E-01$ & $5.3 E-02$ \\
30 & $6.3 E+00$ & $6.3 E-01$ & $6.3 E-02$ \\
62 & $1.2 E+01$ & $1.2 E+00$ & $1.2 E-01$ \\
125 & $1.9 E+01$ & $1.9 E+00$ & $1.9 E-01$ \\
575 & $3.3 E+01$ & $3.3 E+00$ & $3.3 E-01$ \\
750 & $3.3 E+01$ & $3.3 E+00$ & $3.3 E-01$ \\
1000 & $3.3 E+01$ & $3.3 E+00$ & $3.3 E-01$ \\
1025 & $2.8 E+01$ & $2.8 E+00$ & $2.8 E-01$ \\
1050 & $2.4 E+01$ & $2.4 E+00$ & $2.4 E-01$ \\
1100 & $1.7 E+01$ & $1.7 E+00$ & $1.7 E-01$ \\
1200 & $8.3 E+00$ & $8.3 E-01$ & $8.3 E-02$ \\
1400 & $2.1 E+00$ & $2.1 E-01$ & $2.1 E-02$ \\
1600 & $5.2 E-01$ & $5.2 E-02$ & $5.2 E-03$ \\
2000 & $3.2 E-02$ & $3.2 E-03$ & $3.2 E-04$ \\
2500 & $1.0 E-03$ & $1.0 E-04$ & $1.0 E-05$ \\
3000 & $3.2 E-05$ & $3.2 E-06$ & $3.2 E-07$ \\
\hline
\end{tabular}

ABSCM Concentration in Soil ( $\mathrm{mg} \mathrm{\textrm {m } ^ { - 3 }}$ ) in Zone $\mathrm{B}$ Due to Atmospheric Release

\begin{tabular}{rrrr}
\hline Time $(y r)$ & As & $\mathrm{Cr}$ & $\mathrm{Ni}$ \\
\hline 1 & $4.2 \mathrm{E}-02$ & $4.2 \mathrm{E}-03$ & $4.2 \mathrm{E}-04$ \\
20 & $7.8 \mathrm{E}-01$ & $7.8 \mathrm{E}-02$ & $7.8 \mathrm{E}-03$ \\
25 & $9.6 \mathrm{E}-01$ & $9.6 \mathrm{E}-02$ & $9.6 \mathrm{E}-03$ \\
30 & $1.1 \mathrm{E}+00$ & $1.1 \mathrm{E}-01$ & $1.1 \mathrm{E}-02$ \\
62 & $2.1 \mathrm{E}+00$ & $2.1 \mathrm{E}-01$ & $2.1 \mathrm{E}-02$ \\
125 & $3.5 \mathrm{E}+00$ & $3.5 \mathrm{E}-01$ & $3.5 \mathrm{E}-02$ \\
575 & $5.9 \mathrm{E}+00$ & $5.9 \mathrm{E}-01$ & $5.9 \mathrm{E}-02$ \\
750 & $6.0 \mathrm{E}+00$ & $6.0 \mathrm{E}-01$ & $6.0 \mathrm{E}-02$ \\
1000 & $6.0 \mathrm{E}+00$ & $6.0 \mathrm{E}-01$ & $6.0 \mathrm{E}-02$ \\
1025 & $5.1 \mathrm{E}+00$ & $5.1 \mathrm{E}-01$ & $5.1 \mathrm{E}-02$ \\
1050 & $4.3 \mathrm{E}+00$ & $4.3 \mathrm{E}-01$ & $4.3 \mathrm{E}-02$ \\
1100 & $3.0 \mathrm{E}+00$ & $3.0 \mathrm{E}-01$ & $3.0 \mathrm{E}-02$ \\
1200 & $1.5 \mathrm{E}+00$ & $1.5 \mathrm{E}-01$ & $1.5 \mathrm{E}-02$ \\
1400 & $3.8 \mathrm{E}-01$ & $3.8 \mathrm{E}-02$ & $3.8 \mathrm{E}-03$ \\
1600 & $9.4 \mathrm{E}-02$ & $9.4 \mathrm{E}-03$ & $9.4 \mathrm{E}-04$ \\
2000 & $5.9 \mathrm{E}-03$ & $5.9 \mathrm{E}-04$ & $5.9 \mathrm{E}-05$ \\
2500 & $1.8 \mathrm{E}-04$ & $1.8 \mathrm{E}-05$ & $1.8 \mathrm{E}-06$ \\
3000 & $5.7 \mathrm{E}-06$ & $5.7 \mathrm{E}-07$ & $5.7 \mathrm{E}-08$
\end{tabular}


GAWCU Concentration in well water $\left(\mathrm{Bq} \mathrm{L} \mathrm{L}^{-1}\right)$ in zone $\mathrm{A}$ Due to Groundwater Release

\begin{tabular}{rccccc}
\hline Time (yr) & $\mathrm{U}-238 \& 4$ & Th-230 & $\mathrm{Ra}-226$ & $\mathrm{~Pb}-210$ & $\mathrm{PO}-210$ \\
\hline 15 & $0.0 \mathrm{E}+00$ & $0.0 \mathrm{E}+00$ & $0.0 \mathrm{E}+00$ & $0.0 \mathrm{E}+00$ & $0.0 \mathrm{E}+00$ \\
20 & $0.0 \mathrm{E}+00$ & $0.0 \mathrm{E}+00$ & $0.0 \mathrm{E}+00$ & $0.0 \mathrm{E}+00$ & $0.0 \mathrm{E}+00$ \\
25 & $0.0 \mathrm{E}+00$ & $0.0 \mathrm{E}+00$ & $0.0 \mathrm{E}+00$ & $0.0 \mathrm{E}+00$ & $0.0 \mathrm{E}+00$ \\
30 & $0.0 \mathrm{E}+00$ & $0.0 \mathrm{E}+00$ & $0.0 \mathrm{E}+00$ & $0.0 \mathrm{E}+00$ & $0.0 \mathrm{E}+00$ \\
60 & $0.0 \mathrm{E}+00$ & $0.0 \mathrm{E}+00$ & $0.0 \mathrm{E}+00$ & $0.0 \mathrm{E}+00$ & $0.0 \mathrm{E}+00$ \\
62 & $9.1 \mathrm{E}-01$ & $3.6 \mathrm{E}-09$ & $8.3 \mathrm{E}-10$ & $7.8 \mathrm{E}-10$ & $7.8 \mathrm{E}-10$ \\
80 & $5.0 \mathrm{E}+01$ & $1.1 \mathrm{E}-05$ & $6.6 \mathrm{E}-07$ & $6.2 \mathrm{E}-07$ & $6.2 \mathrm{E}-07$ \\
100 & $1.0 \mathrm{E}+02$ & $4.8 \mathrm{E}-05$ & $5.8 \mathrm{E}-06$ & $5.5 \mathrm{E}-06$ & $5.5 \mathrm{E}-06$ \\
125 & $1.7 \mathrm{E}+02$ & $1.3 \mathrm{E}-04$ & $2.6 \mathrm{E}-05$ & $2.5 \mathrm{E}-05$ & $2.5 \mathrm{E}-05$ \\
575 & $1.7 \mathrm{E}+02$ & $1.9 \mathrm{E}-03$ & $4.2 \mathrm{E}-03$ & $3.9 \mathrm{E}-03$ & $3.9 \mathrm{E}-03$ \\
590 & $1.7 \mathrm{E}+02$ & $2.0 \mathrm{E}-03$ & $7.5 \mathrm{E}-01$ & $7.6 \mathrm{E}-01$ & $7.6 \mathrm{E}-01$ \\
620 & $1.7 \mathrm{E}+02$ & $2.1 \mathrm{E}-03$ & $7.4 \mathrm{E}+00$ & $7.5 \mathrm{E}+00$ & $7.5 \mathrm{E}+00$ \\
750 & $1.7 \mathrm{E}+02$ & $2.6 \mathrm{E}-03$ & $3.5 \mathrm{E}+01$ & $3.6 \mathrm{E}+01$ & $3.6 \mathrm{E}+01$ \\
1000 & $1.7 \mathrm{E}+02$ & $3.7 \mathrm{E}-03$ & $8.5 \mathrm{E}+01$ & $8.6 \mathrm{E}+01$ & $8.6 \mathrm{E}+01$ \\
1015 & $1.7 \mathrm{E}+02$ & $3.7 \mathrm{E}-03$ & $8.7 \mathrm{E}+01$ & $8.9 \mathrm{E}+01$ & $8.9 \mathrm{E}+01$ \\
1025 & $1.7 \mathrm{E}+02$ & $3.8 \mathrm{E}-03$ & $8.9 \mathrm{E}+01$ & $9.0 \mathrm{E}+01$ & $9.0 \mathrm{E}+01$ \\
1060 & $1.7 \mathrm{E}+02$ & $3.9 \mathrm{E}-03$ & $9.6 \mathrm{E}+01$ & $9.7 \mathrm{E}+01$ & $9.7 \mathrm{E}+01$ \\
1080 & $1.7 \mathrm{E}+02$ & $4.0 \mathrm{E}-03$ & $9.9 \mathrm{E}+01$ & $1.0 \mathrm{E}+02$ & $1.0 \mathrm{E}+02$ \\
1120 & $9.0 \mathrm{E}+00$ & $4.0 \mathrm{E}-03$ & $1.1 \mathrm{E}+02$ & $1.1 \mathrm{E}+02$ & $1.1 \mathrm{E}+02$ \\
1175 & $0.0 \mathrm{E}+00$ & $4.0 \mathrm{E}-03$ & $1.2 \mathrm{E}+02$ & $1.2 \mathrm{E}+02$ & $1.2 \mathrm{E}+02$ \\
1575 & $0.0 \mathrm{E}+00$ & $4.0 \mathrm{E}-03$ & $1.2 \mathrm{E}+02$ & $1.2 \mathrm{E}+02$ & $1.2 \mathrm{E}+02$ \\
1750 & $0.0 \mathrm{E}+00$ & $4.0 \mathrm{E}-03$ & $8.2 \mathrm{E}+01$ & $8.4 \mathrm{E}+01$ & $8.4 \mathrm{E}+01$ \\
2175 & $0.0 \mathrm{E}+00$ & $3.9 \mathrm{E}-03$ & $2.0 \mathrm{E}+00$ & $3.0 \mathrm{E}+00$ & $3.0 \mathrm{E}+00$ \\
4000 & $0.0 \mathrm{E}+00$ & $3.8 \mathrm{E}-03$ & $3.0 \mathrm{E}+00$ & $3.0 \mathrm{E}+00$ & $2.0 \mathrm{E}+00$ \\
6000 & $0.0 \mathrm{E}+00$ & $3.7 \mathrm{E}-03$ & $3.0 \mathrm{E}+00$ & $3.0 \mathrm{E}+00$ & $3.0 \mathrm{E}+00$ \\
8000 & $0.0 \mathrm{E}+00$ & $3.6 \mathrm{E}-03$ & $3.0 \mathrm{E}+00$ & $3.0 \mathrm{E}+00$ & $3.0 \mathrm{E}+00$ \\
10000 & $0.0 \mathrm{E}+00$ & $3.6 \mathrm{E}-03$ & $4.0 \mathrm{E}+00$ & $3.0 \mathrm{E}+00$ & $3.0 \mathrm{E}+00$ \\
& & & & & \\
\hline
\end{tabular}


GAWCM Concentration in Well

Water ( $\mathrm{mg} \mathrm{L} \mathrm{L}^{-1}$ ) in Zone $\mathrm{A}$ Due to

Groundwater Release

\begin{tabular}{rrrr}
\hline Time $(\mathrm{Yr})$ & As & $\mathrm{Cr}$ & $\mathrm{Ni}$ \\
\hline 15 & $0.0 \mathrm{E}+00$ & $2.6 \mathrm{E}-05$ & $2.6 \mathrm{E}-06$ \\
20 & $0.0 \mathrm{E}+00$ & $2.0 \mathrm{E}+00$ & $2.0 \mathrm{E}-01$ \\
25 & $0.0 \mathrm{E}+00$ & $4.0 \mathrm{E}+00$ & $4.0 \mathrm{E}-01$ \\
30 & $0.0 \mathrm{E}+00$ & $6.0 \mathrm{E}+00$ & $6.0 \mathrm{E}-01$ \\
60 & $0.0 \mathrm{E}+00$ & $6.0 \mathrm{E}+00$ & $6.0 \mathrm{E}-01$ \\
62 & $0.0 \mathrm{E}+00$ & $6.0 \mathrm{E}+00$ & $6.0 \mathrm{E}-01$ \\
80 & $0.0 \mathrm{E}+00$ & $6.0 \mathrm{E}+00$ & $6.0 \mathrm{E}-01$ \\
100 & $0.0 \mathrm{E}+00$ & $6.0 \mathrm{E}+00$ & $6.0 \mathrm{E}-01$ \\
125 & $0.0 \mathrm{E}+00$ & $6.0 \mathrm{E}+00$ & $6.0 \mathrm{E}-01$ \\
575 & $0.0 \mathrm{E}+00$ & $6.0 \mathrm{E}+00$ & $6.0 \mathrm{E}-01$ \\
590 & $0.0 \mathrm{E}+00$ & $6.0 \mathrm{E}+00$ & $6.0 \mathrm{E}-01$ \\
620 & $0.0 \mathrm{E}+00$ & $6.0 \mathrm{E}+00$ & $6.0 \mathrm{E}-01$ \\
750 & $0.0 \mathrm{E}+00$ & $6.0 \mathrm{E}+00$ & $6.0 \mathrm{E}-01$ \\
1000 & $0.0 \mathrm{E}+00$ & $6.0 \mathrm{E}+00$ & $6.0 \mathrm{E}-01$ \\
1015 & $0.0 \mathrm{E}+00$ & $5.9 \mathrm{E}+00$ & $5.9 \mathrm{E}-01$ \\
1025 & $0.0 \mathrm{E}+00$ & $2.0 \mathrm{E}+00$ & $2.0 \mathrm{E}-01$ \\
1060 & $0.0 \mathrm{E}+00$ & $0.0 \mathrm{E}+00$ & $0.0 \mathrm{E}+00$ \\
1080 & $0.0 \mathrm{E}+00$ & $0.0 \mathrm{E}+00$ & $0.0 \mathrm{E}+00$ \\
1120 & $0.0 \mathrm{E}+00$ & $0.0 \mathrm{E}+00$ & $0.0 \mathrm{E}+00$ \\
1175 & $0.0 \mathrm{E}+00$ & $0.0 \mathrm{E}+00$ & $0.0 \mathrm{E}+00$ \\
1575 & $0.0 \mathrm{E}+00$ & $0.0 \mathrm{E}+00$ & $0.0 \mathrm{E}+00$ \\
1750 & $0.0 \mathrm{E}+00$ & $0.0 \mathrm{E}+00$ & $0.0 \mathrm{E}+00$ \\
2175 & $0.0 \mathrm{E}+00$ & $0.0 \mathrm{E}+00$ & $0.0 \mathrm{E}+00$ \\
4000 & $0.0 \mathrm{E}+00$ & $0.0 \mathrm{E}+00$ & $0.0 \mathrm{E}+00$ \\
6000 & $3.4 \mathrm{E}-03$ & $0.0 \mathrm{E}+00$ & $0.0 \mathrm{E}+00$ \\
8000 & $2.1 \mathrm{E}-02$ & $0.0 \mathrm{E}+00$ & $0.0 \mathrm{E}+00$ \\
10000 & $2.1 \mathrm{E}-02$ & $0.0 \mathrm{E}+00$ & $0.0 \mathrm{E}+00$ \\
& & & \\
\hline & & &
\end{tabular}


GAWDU Dose from Drinking water in Zone A Due to Groundwater Release (Sv $\mathrm{yr}^{-1}$ )

\begin{tabular}{rllllll}
\hline Time (yr) & $\mathrm{U}-238 \& 4$ & Th-230 & Ra-226 & Pb-210 & Po-210 & Chain \\
\hline 15 & $0.0 \mathrm{E}+00$ & $0.0 \mathrm{E}+00$ & $0.0 \mathrm{E}+00$ & $0.0 \mathrm{E}+00$ & $0.0 \mathrm{E}+00$ & $0.0 \mathrm{E}+00$ \\
20 & $0.0 \mathrm{E}+00$ & $0.0 \mathrm{E}+00$ & $0.0 \mathrm{E}+00$ & $0.0 \mathrm{E}+00$ & $0.0 \mathrm{E}+00$ & $0.0 \mathrm{E}+00$ \\
25 & $0.0 \mathrm{E}+00$ & $0.0 \mathrm{E}+00$ & $0.0 \mathrm{E}+00$ & $0.0 \mathrm{E}+00$ & $0.0 \mathrm{E}+00$ & $0.0 \mathrm{E}+00$ \\
30 & $0.0 \mathrm{E}+00$ & $0.0 \mathrm{E}+00$ & $0.0 \mathrm{E}+00$ & $0.0 \mathrm{E}+00$ & $0.0 \mathrm{E}+00$ & $0.0 \mathrm{E}+00$ \\
60 & $0.0 \mathrm{E}+00$ & $0.0 \mathrm{E}+00$ & $0.0 \mathrm{E}+00$ & $0.0 \mathrm{E}+00$ & $0.0 \mathrm{E}+00$ & $0.0 \mathrm{E}+00$ \\
62 & $2.7 \mathrm{E}-05$ & $9.2 \mathrm{E}-13$ & $1.3 \mathrm{E}-13$ & $7.4 \mathrm{E}-13$ & $3.5 \mathrm{E}-13$ & $2.7 \mathrm{E}-05$ \\
80 & $1.5 \mathrm{E}-03$ & $2.8 \mathrm{E}-09$ & $1.1 \mathrm{E}-10$ & $5.9 \mathrm{E}-10$ & $2.8 \mathrm{E}-10$ & $1.5 \mathrm{E}-03$ \\
100 & $3.1 \mathrm{E}-03$ & $1.2 \mathrm{E}-08$ & $9.4 \mathrm{E}-10$ & $5.2 \mathrm{E}-09$ & $2.5 \mathrm{E}-09$ & $3.1 \mathrm{E}-03$ \\
125 & $5.0 \mathrm{E}-03$ & $3.3 \mathrm{E}-08$ & $4.3 \mathrm{E}-09$ & $2.4 \mathrm{E}-08$ & $1.1 \mathrm{E}-08$ & $5.0 \mathrm{E}-03$ \\
575 & $5.0 \mathrm{E}-03$ & $4.9 \mathrm{E}-07$ & $6.7 \mathrm{E}-07$ & $3.7 \mathrm{E}-06$ & $1.8 \mathrm{E}-06$ & $5.0 \mathrm{E}-03$ \\
590 & $5.0 \mathrm{E}-03$ & $5.1 \mathrm{E}-07$ & $1.2 \mathrm{E}-04$ & $7.2 \mathrm{E}-04$ & $3.4 \mathrm{E}-04$ & $6.1 \mathrm{E}-03$ \\
620 & $5.0 \mathrm{E}-03$ & $5.4 \mathrm{E}-07$ & $1.2 \mathrm{E}-03$ & $7.1 \mathrm{E}-03$ & $3.4 \mathrm{E}-03$ & $1.7 \mathrm{E}-02$ \\
750 & $5.0 \mathrm{E}-03$ & $6.7 \mathrm{E}-07$ & $5.6 \mathrm{E}-03$ & $3.4 \mathrm{E}-02$ & $1.6 \mathrm{E}-02$ & $6.1 \mathrm{E}-02$ \\
1000 & $5.0 \mathrm{E}-03$ & $9.4 \mathrm{E}-07$ & $1.4 \mathrm{E}-02$ & $8.1 \mathrm{E}-02$ & $3.9 \mathrm{E}-02$ & $1.4 \mathrm{E}-01$ \\
1015 & $5.0 \mathrm{E}-03$ & $9.5 \mathrm{E}-07$ & $1.4 \mathrm{E}-02$ & $8.4 \mathrm{E}-02$ & $4.0 \mathrm{E}-02$ & $1.4 \mathrm{E}-01$ \\
1025 & $5.0 \mathrm{E}-03$ & $9.6 \mathrm{E}-07$ & $1.4 \mathrm{E}-02$ & $8.6 \mathrm{E}-02$ & $4.1 \mathrm{E}-02$ & $1.5 \mathrm{E}-01$ \\
1060 & $5.0 \mathrm{E}-03$ & $1.0 \mathrm{E}-06$ & $1.5 \mathrm{E}-02$ & $9.2 \mathrm{E}-02$ & $4.4 \mathrm{E}-02$ & $1.6 \mathrm{E}-01$ \\
1080 & $4.9 \mathrm{E}-03$ & $1.0 \mathrm{E}-06$ & $1.6 \mathrm{E}-02$ & $9.6 \mathrm{E}-02$ & $4.6 \mathrm{E}-02$ & $1.6 \mathrm{E}-01$ \\
1120 & $2.7 \mathrm{E}-04$ & $1.0 \mathrm{E}-06$ & $1.7 \mathrm{E}-02$ & $1.0 \mathrm{E}-01$ & $4.9 \mathrm{E}-02$ & $1.7 \mathrm{E}-01$ \\
1175 & $0.0 \mathrm{E}+00$ & $1.0 \mathrm{E}-06$ & $1.9 \mathrm{E}-02$ & $1.1 \mathrm{E}-01$ & $5.3 \mathrm{E}-02$ & $1.8 \mathrm{E}-01$ \\
1575 & $0.0 \mathrm{E}+00$ & $1.0 \mathrm{E}-06$ & $1.9 \mathrm{E}-02$ & $1.1 \mathrm{E}-01$ & $5.4 \mathrm{E}-02$ & $1.8 \mathrm{E}-01$ \\
1750 & $0.0 \mathrm{E}+00$ & $1.0 \mathrm{E}-06$ & $1.3 \mathrm{E}-02$ & $7.9 \mathrm{E}-02$ & $3.8 \mathrm{E}-02$ & $1.3 \mathrm{E}-01$ \\
2175 & $0.0 \mathrm{E}+00$ & $1.0 \mathrm{E}-06$ & $4.0 \mathrm{E}-04$ & $3.0 \mathrm{E}-03$ & $1.3 \mathrm{E}-03$ & $4.7 \mathrm{E}-03$ \\
4000 & $0.0 \mathrm{E}+00$ & $9.8 \mathrm{E}-07$ & $4.0 \mathrm{E}-04$ & $3.0 \mathrm{E}-03$ & $1.3 \mathrm{E}-03$ & $4.7 \mathrm{E}-03$ \\
6000 & $0.0 \mathrm{E}+00$ & $9.6 \mathrm{E}-07$ & $4.0 \mathrm{E}-04$ & $3.0 \mathrm{E}-03$ & $1.4 \mathrm{E}-03$ & $4.8 \mathrm{E}-03$ \\
8000 & $0.0 \mathrm{E}+00$ & $9.5 \mathrm{E}-07$ & $5.0 \mathrm{E}-04$ & $3.0 \mathrm{E}-03$ & $1.3 \mathrm{E}-03$ & $4.8 \mathrm{E}-03$ \\
10000 & $0.0 \mathrm{E}+00$ & $9.2 \mathrm{E}-07$ & $4.0 \mathrm{E}-04$ & $3.0 \mathrm{E}-03$ & $1.4 \mathrm{E}-03$ & $4.8 \mathrm{E}-03$ \\
& & & & & & \\
\hline & & & & & & \\
\hline
\end{tabular}


GAWIM Intake from Drinking water in Zone A Due to Groundwater Release ( $m g \mathrm{yr}^{-1}$ )

\begin{tabular}{rrrr}
\hline Time (Yr) & As & $\mathrm{Cr}$ & $\mathrm{Ni}$ \\
\hline 15 & $0.0 \mathrm{E}+00$ & $1.9 \mathrm{E}-02$ & $1.9 \mathrm{E}-03$ \\
20 & $0.0 \mathrm{E}+00$ & $1.5 \mathrm{E}+03$ & $1.5 \mathrm{E}+02$ \\
25 & $0.0 \mathrm{E}+00$ & $2.9 \mathrm{E}+03$ & $2.9 \mathrm{E}+02$ \\
30 & $0.0 \mathrm{E}+00$ & $4.4 \mathrm{E}+03$ & $4.4 \mathrm{E}+02$ \\
60 & $0.0 \mathrm{E}+00$ & $4.4 \mathrm{E}+03$ & $4.4 \mathrm{E}+02$ \\
62 & $0.0 \mathrm{E}+00$ & $4.4 \mathrm{E}+03$ & $4.4 \mathrm{E}+02$ \\
80 & $0.0 \mathrm{E}+00$ & $4.4 \mathrm{E}+03$ & $4.4 \mathrm{E}+02$ \\
100 & $0.0 \mathrm{E}+00$ & $4.4 \mathrm{E}+03$ & $4.4 \mathrm{E}+02$ \\
125 & $0.0 \mathrm{E}+00$ & $4.4 \mathrm{E}+03$ & $4.4 \mathrm{E}+02$ \\
575 & $0.0 \mathrm{E}+00$ & $4.4 \mathrm{E}+03$ & $4.4 \mathrm{E}+02$ \\
590 & $0.0 \mathrm{E}+00$ & $4.4 \mathrm{E}+03$ & $4.4 \mathrm{E}+02$ \\
620 & $0.0 \mathrm{E}+00$ & $4.4 \mathrm{E}+03$ & $4.4 \mathrm{E}+02$ \\
750 & $0.0 \mathrm{E}+00$ & $4.4 \mathrm{E}+03$ & $4.4 \mathrm{E}+02$ \\
1000 & $0.0 \mathrm{E}+00$ & $4.3 \mathrm{E}+03$ & $4.3 \mathrm{E}+02$ \\
1015 & $0.0 \mathrm{E}+00$ & $4.3 \mathrm{E}+03$ & $4.3 \mathrm{E}+02$ \\
1025 & $0.0 \mathrm{E}+00$ & $1.4 \mathrm{E}+03$ & $1.4 \mathrm{E}+02$ \\
1060 & $0.0 \mathrm{E}+00$ & $0.0 \mathrm{E}+00$ & $0.0 \mathrm{E}+00$ \\
1080 & $0.0 \mathrm{E}+00$ & $0.0 \mathrm{E}+00$ & $0.0 \mathrm{E}+00$ \\
1120 & $0.0 \mathrm{E}+00$ & $0.0 \mathrm{E}+00$ & $0.0 \mathrm{E}+00$ \\
1175 & $0.0 \mathrm{E}+00$ & $0.0 \mathrm{E}+00$ & $0.0 \mathrm{E}+00$ \\
1575 & $0.0 \mathrm{E}+00$ & $0.0 \mathrm{E}+00$ & $0.0 \mathrm{E}+00$ \\
1750 & $0.0 \mathrm{E}+00$ & $0.0 \mathrm{E}+00$ & $0.0 \mathrm{E}+00$ \\
2175 & $0.0 \mathrm{E}+00$ & $0.0 \mathrm{E}+00$ & $0.0 \mathrm{E}+00$ \\
4000 & $0.0 \mathrm{E}+00$ & $0.0 \mathrm{E}+00$ & $0.0 \mathrm{E}+00$ \\
6000 & $2.5 \mathrm{E}+00$ & $0.0 \mathrm{E}+00$ & $0.0 \mathrm{E}+00$ \\
8000 & $1.5 \mathrm{E}+01$ & $0.0 \mathrm{E}+00$ & $0.0 \mathrm{E}+00$ \\
10000 & $1.5 \mathrm{E}+01$ & $0.0 \mathrm{E}+00$ & $0.0 \mathrm{E}+00$ \\
& & & \\
\hline & & &
\end{tabular}


GAWRU Cancer Risk from Drinking Water in Zone A

Due to Groundwater Release

\begin{tabular}{rlllllll}
\hline Time (Yr) & $\mathrm{U}-238 \& 4$ & $\mathrm{Th}-230$ & $\mathrm{Ra}-226$ & $\mathrm{~Pb}-210$ & Po-210 & Chain \\
\hline 15 & $0.0 \mathrm{E}+00$ & $0.0 \mathrm{E}+00$ & $0.0 \mathrm{E}+00$ & $0.0 \mathrm{E}+00$ & $0.0 \mathrm{E}+00$ & $0.0 \mathrm{E}+00$ \\
20 & $0.0 \mathrm{E}+00$ & $0.0 \mathrm{E}+00$ & $0.0 \mathrm{E}+00$ & $0.0 \mathrm{E}+00$ & $0.0 \mathrm{E}+00$ & $0.0 \mathrm{E}+00$ \\
25 & $0.0 \mathrm{E}+00$ & $0.0 \mathrm{E}+00$ & $0.0 \mathrm{E}+00$ & $0.0 \mathrm{E}+00$ & $0.0 \mathrm{E}+00$ & $0.0 \mathrm{E}+00$ \\
30 & $0.0 \mathrm{E}+00$ & $0.0 \mathrm{E}+00$ & $0.0 \mathrm{E}+00$ & $0.0 \mathrm{E}+00$ & $0.0 \mathrm{E}+00$ & $0.0 \mathrm{E}+00$ \\
60 & $0.0 \mathrm{E}+00$ & $0.0 \mathrm{E}+00$ & $0.0 \mathrm{E}+00$ & $0.0 \mathrm{E}+00$ & $0.0 \mathrm{E}+00$ & $0.0 \mathrm{E}+00$ \\
62 & $2.8 \mathrm{E}-05$ & $6.4 \mathrm{E}-14$ & $1.4 \mathrm{E}-13$ & $5.6 \mathrm{E}-13$ & $1.6 \mathrm{E}-13$ & $2.8 \mathrm{E}-05$ \\
80 & $1.5 \mathrm{E}-03$ & $2.0 \mathrm{E}-10$ & $1.1 \mathrm{E}-10$ & $4.4 \mathrm{E}-10$ & $1.3 \mathrm{E}-10$ & $1.5 \mathrm{E}-03$ \\
100 & $3.2 \mathrm{E}-03$ & $8.6 \mathrm{E}-10$ & $9.5 \mathrm{E}-10$ & $3.9 \mathrm{E}-09$ & $1.1 \mathrm{E}-09$ & $3.2 \mathrm{E}-03$ \\
125 & $5.1 \mathrm{E}-03$ & $2.3 \mathrm{E}-09$ & $4.3 \mathrm{E}-09$ & $1.8 \mathrm{E}-08$ & $5.2 \mathrm{E}-09$ & $5.1 \mathrm{E}-03$ \\
575 & $5.1 \mathrm{E}-03$ & $3.5 \mathrm{E}-08$ & $6.8 \mathrm{E}-07$ & $2.8 \mathrm{E}-06$ & $8.2 \mathrm{E}-07$ & $5.1 \mathrm{E}-03$ \\
590 & $5.1 \mathrm{E}-03$ & $3.6 \mathrm{E}-08$ & $1.2 \mathrm{E}-04$ & $5.4 \mathrm{E}-04$ & $1.6 \mathrm{E}-04$ & $5.9 \mathrm{E}-03$ \\
620 & $5.1 \mathrm{E}-03$ & $3.8 \mathrm{E}-08$ & $1.2 \mathrm{E}-03$ & $5.3 \mathrm{E}-03$ & $1.6 \mathrm{E}-03$ & $1.3 \mathrm{E}-02$ \\
750 & $5.1 \mathrm{E}-03$ & $4.7 \mathrm{E}-08$ & $5.7 \mathrm{E}-03$ & $2.5 \mathrm{E}-02$ & $7.5 \mathrm{E}-03$ & $4.4 \mathrm{E}-02$ \\
1000 & $5.1 \mathrm{E}-03$ & $6.6 \mathrm{E}-08$ & $1.4 \mathrm{E}-02$ & $6.1 \mathrm{E}-02$ & $1.8 \mathrm{E}-02$ & $9.8 \mathrm{E}-02$ \\
1015 & $5.1 \mathrm{E}-03$ & $6.7 \mathrm{E}-08$ & $1.4 \mathrm{E}-02$ & $6.3 \mathrm{E}-02$ & $1.9 \mathrm{E}-02$ & $1.0 \mathrm{E}-01$ \\
1025 & $5.1 \mathrm{E}-03$ & $6.7 \mathrm{E}-08$ & $1.5 \mathrm{E}-02$ & $6.5 \mathrm{E}-02$ & $1.9 \mathrm{E}-02$ & $1.0 \mathrm{E}-01$ \\
1060 & $5.1 \mathrm{E}-03$ & $7.0 \mathrm{E}-08$ & $1.6 \mathrm{E}-02$ & $6.9 \mathrm{E}-02$ & $2.0 \mathrm{E}-02$ & $1.1 \mathrm{E}-01$ \\
1080 & $5.1 \mathrm{E}-03$ & $7.1 \mathrm{E}-08$ & $1.6 \mathrm{E}-02$ & $7.2 \mathrm{E}-02$ & $2.1 \mathrm{E}-02$ & $1.1 \mathrm{E}-01$ \\
1120 & $2.8 \mathrm{E}-04$ & $7.2 \mathrm{E}-08$ & $1.7 \mathrm{E}-02$ & $7.7 \mathrm{E}-02$ & $2.3 \mathrm{E}-02$ & $1.2 \mathrm{E}-01$ \\
1175 & $1.0 \mathrm{E}-06$ & $7.2 \mathrm{E}-08$ & $1.9 \mathrm{E}-02$ & $8.4 \mathrm{E}-02$ & $2.5 \mathrm{E}-02$ & $1.3 \mathrm{E}-01$ \\
1575 & $2.0 \mathrm{E}-06$ & $7.1 \mathrm{E}-08$ & $1.9 \mathrm{E}-02$ & $8.5 \mathrm{E}-02$ & $2.5 \mathrm{E}-02$ & $1.3 \mathrm{E}-01$ \\
1750 & $0.0 \mathrm{E}+00$ & $7.1 \mathrm{E}-08$ & $1.3 \mathrm{E}-02$ & $6.0 \mathrm{E}-02$ & $1.7 \mathrm{E}-02$ & $9.1 \mathrm{E}-02$ \\
2175 & $1.0 \mathrm{E}-06$ & $6.9 \mathrm{E}-08$ & $4.4 \mathrm{E}-04$ & $2.0 \mathrm{E}-03$ & $5.7 \mathrm{E}-04$ & $3.0 \mathrm{E}-03$ \\
4000 & $2.0 \mathrm{E}-06$ & $6.8 \mathrm{E}-08$ & $4.4 \mathrm{E}-04$ & $2.0 \mathrm{E}-03$ & $5.8 \mathrm{E}-04$ & $3.0 \mathrm{E}-03$ \\
6000 & $0.0 \mathrm{E}+00$ & $6.7 \mathrm{E}-08$ & $4.5 \mathrm{E}-04$ & $2.2 \mathrm{E}-03$ & $6.5 \mathrm{E}-04$ & $3.3 \mathrm{E}-03$ \\
8000 & $1.0 \mathrm{E}-06$ & $6.6 \mathrm{E}-08$ & $4.8 \mathrm{E}-04$ & $2.1 \mathrm{E}-03$ & $6.2 \mathrm{E}-04$ & $3.2 \mathrm{E}-03$ \\
10000 & $1.0 \mathrm{E}-06$ & $6.5 \mathrm{E}-08$ & $5.1 \mathrm{E}-04$ & $2.2 \mathrm{E}-03$ & $6.5 \mathrm{E}-04$ & $3.4 \mathrm{E}-03$ \\
& & & & & & & \\
\hline & & & & & & \\
\hline
\end{tabular}


GAWRM Cancer Risk from Drinking Water in Zone A Due to Groundwater Release

\begin{tabular}{|c|c|c|c|}
\hline Time (yr) & As & $\mathrm{Cr}$ & $\mathrm{Ni}$ \\
\hline 15 & $0.0 E+00$ & $0.0 E+00$ & $0.0 E+00$ \\
\hline 20 & $0.0 E+00$ & $0.0 E+00$ & $0.0 E+00$ \\
\hline 25 & $0.0 E+00$ & $0.0 E+00$ & $0.0 E+00$ \\
\hline 30 & $0.0 E+00$ & $0.0 \mathrm{E}+00$ & $0.0 E+00$ \\
\hline 60 & $0.0 E+00$ & $0.0 E+00$ & $0.0 \mathrm{E}+00$ \\
\hline 62 & $0.0 E+00$ & $0.0 E+00$ & $0.0 \mathrm{E}+00$ \\
\hline 80 & $0.0 E+00$ & $0.0 E+00$ & $0.0 \mathrm{E}+00$ \\
\hline 100 & $0.0 E+00$ & $0.0 E+00$ & $0.0 \mathrm{E}+00$ \\
\hline 125 & $0.0 E+00$ & $0.0 E+00$ & $0.0 \mathrm{E}+00$ \\
\hline 575 & $0.0 E+00$ & $0.0 E+00$ & $0.0 \mathrm{E}+00$ \\
\hline 590 & $0.0 E+00$ & $0.0 E+00$ & $0.0 \mathrm{E}+00$ \\
\hline 620 & $0.0 E+00$ & $0.0 E+00$ & $0.0 \mathrm{E}+00$ \\
\hline 750 & $0.0 E+00$ & $0.0 E+00$ & $0.0 E+00$ \\
\hline 1000 & $0.0 E+00$ & $0.0 E+00$ & $0.0 \mathrm{E}+00$ \\
\hline 1015 & $0.0 E+00$ & $0.0 E+00$ & $0.0 E+00$ \\
\hline 1025 & $0.0 E+00$ & $0.0 E+00$ & $0.0 E+00$ \\
\hline 1060 & $0.0 \mathrm{E}+00$ & $0.0 E+00$ & $0.0 \mathrm{E}+00$ \\
\hline 1080 & $0.0 E+00$ & $0.0 E+00$ & $0.0 E+00$ \\
\hline 1120 & $0.0 E+00$ & $0.0 E+00$ & $0.0 \mathrm{E}+00$ \\
\hline 1175 & $0.0 E+00$ & $0.0 E+00$ & $0.0 \mathrm{E}+00$ \\
\hline 1575 & $0.0 E+00$ & $0.0 E+00$ & $0.0 \mathrm{E}+00$ \\
\hline 1750 & $0.0 E+00$ & $0.0 E+00$ & $0.0 E+00$ \\
\hline 2175 & $0.0 E+00$ & $0.0 E+00$ & $0.0 \mathrm{E}+00$ \\
\hline 4000 & $0.0 E+00$ & $0.0 E+00$ & $0.0 \mathrm{E}+00$ \\
\hline 6000 & $4.3 E-02$ & $0.0 E+00$ & $0.0 \mathrm{E}+00$ \\
\hline 8000 & 2. $6 \mathrm{E}-01$ & $0.0 \mathrm{E}+00$ & $0.0 \mathrm{E}+00$ \\
\hline 10000 & $2.6 E-01$ & $0.0 \mathrm{E}+00$ & $0.0 \mathrm{E}+00$ \\
\hline
\end{tabular}

\begin{tabular}{rrrrrrrr}
$\begin{array}{l}\text { AATDU } \\
\text { (Sv } \mathrm{Yr}^{-1} \text { ) }\end{array}$ & Total Dose in Zone A Due to Atmospheric Release \\
\hline Time $(\mathrm{Yr})$ & $\mathrm{U}-238 \& 4$ & $\mathrm{Th}-230$ & $\mathrm{Ra}-226$ & $\mathrm{~Pb}-210$ & Po-210 & Chain \\
\hline 1 & $6.9 \mathrm{E}-07$ & $9.7 \mathrm{E}-06$ & $1.6 \mathrm{E}-06$ & $8.3 \mathrm{E}-06$ & $9.8 \mathrm{E}-05$ & $1.2 \mathrm{E}-04$ \\
20 & $6.9 \mathrm{E}-07$ & $9.7 \mathrm{E}-06$ & $2.9 \mathrm{E}-06$ & $8.4 \mathrm{E}-06$ & $1.0 \mathrm{E}-04$ & $1.2 \mathrm{E}-04$ \\
25 & $6.9 \mathrm{E}-07$ & $9.7 \mathrm{E}-06$ & $3.2 \mathrm{E}-06$ & $8.4 \mathrm{E}-06$ & $1.0 \mathrm{E}-04$ & $1.3 \mathrm{E}-04$ \\
30 & $6.9 \mathrm{E}-07$ & $9.6 \mathrm{E}-06$ & $3.5 \mathrm{E}-06$ & $8.4 \mathrm{E}-06$ & $1.0 \mathrm{E}-04$ & $1.3 \mathrm{E}-04$ \\
62 & $7.0 \mathrm{E}-07$ & $9.7 \mathrm{E}-06$ & $5.1 \mathrm{E}-06$ & $8.5 \mathrm{E}-06$ & $1.1 \mathrm{E}-04$ & $1.3 \mathrm{E}-04$ \\
125 & $7.0 \mathrm{E}-07$ & $9.7 \mathrm{E}-06$ & $7.4 \mathrm{E}-06$ & $8.6 \mathrm{E}-06$ & $1.2 \mathrm{E}-04$ & $1.5 \mathrm{E}-04$ \\
575 & $7.1 \mathrm{E}-07$ & $9.6 \mathrm{E}-06$ & $1.1 \mathrm{E}-05$ & $8.8 \mathrm{E}-06$ & $1.4 \mathrm{E}-04$ & $1.7 \mathrm{E}-04$ \\
750 & $7.1 \mathrm{E}-07$ & $9.6 \mathrm{E}-06$ & $1.2 \mathrm{E}-05$ & $8.8 \mathrm{E}-06$ & $1.4 \mathrm{E}-04$ & $1.7 \mathrm{E}-04$ \\
1000 & $7.1 \mathrm{E}-07$ & $9.6 \mathrm{E}-06$ & $1.2 \mathrm{E}-05$ & $8.8 \mathrm{E}-06$ & $1.4 \mathrm{E}-04$ & $1.7 \mathrm{E}-04$ \\
1025 & $2.0 \mathrm{E}-08$ & $2.1 \mathrm{E}-08$ & $8.4 \mathrm{E}-06$ & $4.0 \mathrm{E}-07$ & $3.3 \mathrm{E}-05$ & $4.2 \mathrm{E}-05$ \\
1050 & $1.7 \mathrm{E}-08$ & $1.8 \mathrm{E}-08$ & $7.1 \mathrm{E}-06$ & $3.4 \mathrm{E}-07$ & $2.7 \mathrm{E}-05$ & $3.5 \mathrm{E}-05$ \\
1100 & $1.2 \mathrm{E}-08$ & $1.2 \mathrm{E}-08$ & $5.0 \mathrm{E}-06$ & $2.4 \mathrm{E}-07$ & $1.9 \mathrm{E}-05$ & $2.5 \mathrm{E}-05$ \\
1200 & $6.0 \mathrm{E}-09$ & $6.2 \mathrm{E}-09$ & $2.5 \mathrm{E}-06$ & $1.2 \mathrm{E}-07$ & $9.7 \mathrm{E}-06$ & $1.2 \mathrm{E}-05$ \\
1400 & $1.5 \mathrm{E}-09$ & $1.6 \mathrm{E}-09$ & $6.3 \mathrm{E}-07$ & $3.0 \mathrm{E}-08$ & $2.4 \mathrm{E}-06$ & $3.1 \mathrm{E}-06$ \\
1600 & $3.8 \mathrm{E}-10$ & $3.9 \mathrm{E}-10$ & $1.6 \mathrm{E}-07$ & $7.5 \mathrm{E}-09$ & $6.1 \mathrm{E}-07$ & $7.7 \mathrm{E}-07$ \\
2000 & $2.4 \mathrm{E}-11$ & $2.4 \mathrm{E}-11$ & $9.8 \mathrm{E}-09$ & $4.7 \mathrm{E}-10$ & $3.8 \mathrm{E}-08$ & $4.8 \mathrm{E}-08$ \\
2500 & $7.4 \mathrm{E}-13$ & $7.5 \mathrm{E}-13$ & $3.0 \mathrm{E}-10$ & $1.5 \mathrm{E}-11$ & $1.2 \mathrm{E}-09$ & $1.5 \mathrm{E}-09$ \\
3000 & $2.3 \mathrm{E}-14$ & $2.3 \mathrm{E}-14$ & $9.5 \mathrm{E}-12$ & $4.5 \mathrm{E}-13$ & $3.7 \mathrm{E}-11$ & $4.7 \mathrm{E}-11$ \\
& & & & & & & \\
\hline
\end{tabular}


GATDU Total Dose in Zone A Due to Groundwater Release
$\left(\mathrm{SV} \mathrm{Yr}^{-1}\right)$

\begin{tabular}{rcccccc}
\hline Time (yr) & $\mathrm{U}-238 \& 4$ & Th-230 & Ra-226 & Pb-210 & PO-210 & Chain \\
\hline 15 & $0.0 \mathrm{E}+00$ & $0.0 \mathrm{E}+00$ & $0.0 \mathrm{E}+00$ & $0.0 \mathrm{E}+00$ & $0.0 \mathrm{E}+00$ & $0.0 \mathrm{E}+00$ \\
20 & $0.0 \mathrm{E}+00$ & $0.0 \mathrm{E}+00$ & $0.0 \mathrm{E}+00$ & $0.0 \mathrm{E}+00$ & $0.0 \mathrm{E}+00$ & $0.0 \mathrm{E}+00$ \\
25 & $0.0 \mathrm{E}+00$ & $0.0 \mathrm{E}+00$ & $0.0 \mathrm{E}+00$ & $0.0 \mathrm{E}+00$ & $0.0 \mathrm{E}+00$ & $0.0 \mathrm{E}+00$ \\
30 & $0.0 \mathrm{E}+00$ & $0.0 \mathrm{E}+00$ & $0.0 \mathrm{E}+00$ & $0.0 \mathrm{E}+00$ & $0.0 \mathrm{E}+00$ & $0.0 \mathrm{E}+00$ \\
60 & $0.0 \mathrm{E}+00$ & $0.0 \mathrm{E}+00$ & $0.0 \mathrm{E}+00$ & $0.0 \mathrm{E}+00$ & $0.0 \mathrm{E}+00$ & $0.0 \mathrm{E}+00$ \\
62 & $3.0 \mathrm{E}-05$ & $1.0 \mathrm{E}-12$ & $1.4 \mathrm{E}-13$ & $9.3 \mathrm{E}-13$ & $9.7 \mathrm{E}-13$ & $3.0 \mathrm{E}-05$ \\
80 & $1.6 \mathrm{E}-03$ & $3.0 \mathrm{E}-09$ & $1.1 \mathrm{E}-10$ & $7.4 \mathrm{E}-10$ & $7.7 \mathrm{E}-10$ & $1.6 \mathrm{E}-03$ \\
100 & $3.4 \mathrm{E}-03$ & $1.3 \mathrm{E}-08$ & $9.8 \mathrm{E}-10$ & $6.5 \mathrm{E}-09$ & $6.8 \mathrm{E}-09$ & $3.4 \mathrm{E}-03$ \\
125 & $5.5 \mathrm{E}-03$ & $3.6 \mathrm{E}-08$ & $4.4 \mathrm{E}-09$ & $3.0 \mathrm{E}-08$ & $3.1 \mathrm{E}-08$ & $5.5 \mathrm{E}-03$ \\
575 & $5.5 \mathrm{E}-03$ & $5.4 \mathrm{E}-07$ & $7.0 \mathrm{E}-07$ & $4.6 \mathrm{E}-06$ & $4.9 \mathrm{E}-06$ & $5.5 \mathrm{E}-03$ \\
590 & $5.5 \mathrm{E}-03$ & $5.5 \mathrm{E}-07$ & $1.3 \mathrm{E}-04$ & $9.0 \mathrm{E}-04$ & $9.4 \mathrm{E}-04$ & $7.5 \mathrm{E}-03$ \\
620 & $5.5 \mathrm{E}-03$ & $5.8 \mathrm{E}-07$ & $1.2 \mathrm{E}-03$ & $8.9 \mathrm{E}-03$ & $9.3 \mathrm{E}-03$ & $2.5 \mathrm{E}-02$ \\
750 & $5.5 \mathrm{E}-03$ & $7.3 \mathrm{E}-07$ & $5.9 \mathrm{E}-03$ & $4.2 \mathrm{E}-02$ & $4.4 \mathrm{E}-02$ & $9.8 \mathrm{E}-02$ \\
1000 & $5.5 \mathrm{E}-03$ & $1.0 \mathrm{E}-06$ & $1.4 \mathrm{E}-02$ & $1.0 \mathrm{E}-01$ & $1.1 \mathrm{E}-01$ & $2.3 \mathrm{E}-01$ \\
1015 & $5.5 \mathrm{E}-03$ & $1.0 \mathrm{E}-06$ & $1.5 \mathrm{E}-02$ & $1.1 \mathrm{E}-01$ & $1.1 \mathrm{E}-01$ & $2.4 \mathrm{E}-01$ \\
1025 & $5.5 \mathrm{E}-03$ & $1.0 \mathrm{E}-06$ & $1.5 \mathrm{E}-02$ & $1.1 \mathrm{E}-01$ & $1.1 \mathrm{E}-01$ & $2.4 \mathrm{E}-01$ \\
1060 & $5.5 \mathrm{E}-03$ & $1.1 \mathrm{E}-06$ & $1.6 \mathrm{E}-02$ & $1.2 \mathrm{E}-01$ & $1.2 \mathrm{E}-01$ & $2.6 \mathrm{E}-01$ \\
1080 & $5.5 \mathrm{E}-03$ & $1.1 \mathrm{E}-06$ & $1.7 \mathrm{E}-02$ & $1.2 \mathrm{E}-01$ & $1.3 \mathrm{E}-01$ & $2.7 \mathrm{E}-01$ \\
1120 & $3.0 \mathrm{E}-04$ & $1.1 \mathrm{E}-06$ & $1.8 \mathrm{E}-02$ & $1.3 \mathrm{E}-01$ & $1.3 \mathrm{E}-01$ & $2.8 \mathrm{E}-01$ \\
1175 & $0.0 \mathrm{E}+00$ & $1.1 \mathrm{E}-06$ & $1.9 \mathrm{E}-02$ & $1.4 \mathrm{E}-01$ & $1.5 \mathrm{E}-01$ & $3.1 \mathrm{E}-01$ \\
1575 & $0.0 \mathrm{E}+00$ & $1.1 \mathrm{E}-06$ & $2.0 \mathrm{E}-02$ & $1.4 \mathrm{E}-01$ & $1.5 \mathrm{E}-01$ & $3.1 \mathrm{E}-01$ \\
1750 & $0.0 \mathrm{E}+00$ & $1.1 \mathrm{E}-06$ & $1.4 \mathrm{E}-02$ & $1.0 \mathrm{E}-01$ & $1.0 \mathrm{E}-01$ & $2.2 \mathrm{E}-01$ \\
2175 & $0.0 \mathrm{E}+00$ & $1.1 \mathrm{E}-06$ & $4.2 \mathrm{E}-04$ & $3.6 \mathrm{E}-03$ & $3.5 \mathrm{E}-03$ & $7.5 \mathrm{E}-03$ \\
4000 & $0.0 \mathrm{E}+00$ & $1.1 \mathrm{E}-06$ & $4.2 \mathrm{E}-04$ & $3.6 \mathrm{E}-03$ & $3.6 \mathrm{E}-03$ & $7.6 \mathrm{E}-03$ \\
6000 & $0.0 \mathrm{E}+00$ & $1.0 \mathrm{E}-06$ & $4.2 \mathrm{E}-04$ & $3.7 \mathrm{E}-03$ & $3.9 \mathrm{E}-03$ & $8.0 \mathrm{E}-03$ \\
8000 & $0.0 \mathrm{E}+00$ & $1.0 \mathrm{E}-06$ & $5.2 \mathrm{E}-04$ & $3.7 \mathrm{E}-03$ & $3.5 \mathrm{E}-03$ & $7.7 \mathrm{E}-03$ \\
10000 & $0.0 \mathrm{E}+00$ & $1.0 \mathrm{E}-06$ & $4.2 \mathrm{E}-04$ & $3.7 \mathrm{E}-03$ & $3.5 \mathrm{E}-03$ & $7.6 \mathrm{E}-03$ \\
& & & & & & \\
\hline
\end{tabular}

AATIM Total Intake in Zone A

Due to Atmospheric Release ( $m g \mathrm{yr}^{-1}$ )

\begin{tabular}{rrrr}
\hline Time $(\mathrm{yr})$ & As & $\mathrm{Cr}$ & $\mathrm{Ni}$ \\
& & & \\
20 & $1.1 \mathrm{E}+00$ & $6.2 \mathrm{E}-03$ & $5.1 \mathrm{E}-04$ \\
25 & $1.2 \mathrm{E}+00$ & $6.4 \mathrm{E}-03$ & $5.2 \mathrm{E}-04$ \\
30 & $1.2 \mathrm{E}+00$ & $6.5 \mathrm{E}-03$ & $5.2 \mathrm{E}-04$ \\
62 & $1.3 \mathrm{E}+00$ & $6.8 \mathrm{E}-03$ & $5.3 \mathrm{E}-04$ \\
125 & $1.4 \mathrm{E}+00$ & $7.2 \mathrm{E}-03$ & $5.6 \mathrm{E}-04$ \\
575 & $1.5 \mathrm{E}+00$ & $7.9 \mathrm{E}-03$ & $5.8 \mathrm{E}-04$ \\
750 & $1.5 \mathrm{E}+00$ & $8.0 \mathrm{E}-03$ & $5.8 \mathrm{E}-04$ \\
1000 & $1.5 \mathrm{E}+00$ & $\mathbf{8 . 0 \mathrm { E } - 0 3}$ & $5.8 \mathrm{E}-04$ \\
1025 & $3.5 \mathrm{E}-01$ & $1.5 \mathrm{E}-03$ & $6.3 \mathrm{E}-05$ \\
1050 & $3.0 \mathrm{E}-01$ & $1.3 \mathrm{E}-03$ & $5.3 \mathrm{E}-05$ \\
1100 & $2.1 \mathrm{E}-01$ & $8.9 \mathrm{E}-04$ & $3.7 \mathrm{E}-05$ \\
1200 & $1.0 \mathrm{E}-01$ & $4.5 \mathrm{E}-04$ & $1.9 \mathrm{E}-05$ \\
1400 & $2.6 \mathrm{E}-02$ & $1.1 \mathrm{E}-04$ & $4.6 \mathrm{E}-06$ \\
1600 & $6.5 \mathrm{E}-03$ & $2.8 \mathrm{E}-05$ & $1.2 \mathrm{E}-06$ \\
2000 & $4.1 \mathrm{E}-04$ & $1.7 \mathrm{E}-06$ & $7.3 \mathrm{E}-08$ \\
2500 & $1.3 \mathrm{E}-05$ & $5.4 \mathrm{E}-08$ & $2.3 \mathrm{E}-09$ \\
3000 & $4.0 \mathrm{E}-07$ & $1.7 \mathrm{E}-09$ & $7.1 \mathrm{E}-11$ \\
& & & \\
\hline
\end{tabular}


GATIM Total Intake in zone A Due to Groundwater Release ( $\mathrm{mg} \mathrm{yr}^{-1}$ )

\begin{tabular}{rrrr}
\hline Time (yr) & As & $\mathrm{Cr}$ & $\mathrm{Ni}$ \\
\hline 15 & $0.0 \mathrm{E}+00$ & $2.2 \mathrm{E}-02$ & $2.0 \mathrm{E}-03$ \\
20 & $0.0 \mathrm{E}+00$ & $1.8 \mathrm{E}+03$ & $1.5 \mathrm{E}+02$ \\
25 & $0.0 \mathrm{E}+00$ & $3.5 \mathrm{E}+03$ & $3.1 \mathrm{E}+02$ \\
30 & $0.0 \mathrm{E}+00$ & $5.3 \mathrm{E}+03$ & $4.6 \mathrm{E}+02$ \\
60 & $0.0 \mathrm{E}+00$ & $5.3 \mathrm{E}+03$ & $4.6 \mathrm{E}+02$ \\
62 & $0.0 \mathrm{E}+00$ & $5.3 \mathrm{E}+03$ & $4.6 \mathrm{E}+02$ \\
80 & $0.0 \mathrm{E}+00$ & $5.3 \mathrm{E}+03$ & $4.6 \mathrm{E}+02$ \\
100 & $0.0 \mathrm{E}+00$ & $5.3 \mathrm{E}+03$ & $4.6 \mathrm{E}+02$ \\
125 & $0.0 \mathrm{E}+00$ & $5.3 \mathrm{E}+03$ & $4.6 \mathrm{E}+02$ \\
575 & $0.0 \mathrm{E}+00$ & $5.2 \mathrm{E}+03$ & $4.6 \mathrm{E}+02$ \\
590 & $0.0 \mathrm{E}+00$ & $5.2 \mathrm{E}+03$ & $4.6 \mathrm{E}+02$ \\
620 & $0.0 \mathrm{E}+00$ & $5.2 \mathrm{E}+03$ & $4.6 \mathrm{E}+02$ \\
750 & $0.0 \mathrm{E}+00$ & $5.2 \mathrm{E}+03$ & $4.6 \mathrm{E}+02$ \\
1000 & $0.0 \mathrm{E}+00$ & $5.2 \mathrm{E}+03$ & $4.6 \mathrm{E}+02$ \\
1015 & $0.0 \mathrm{E}+00$ & $5.2 \mathrm{E}+03$ & $4.6 \mathrm{E}+02$ \\
1025 & $0.0 \mathrm{E}+00$ & $1.7 \mathrm{E}+03$ & $1.5 \mathrm{E}+02$ \\
1060 & $0.0 \mathrm{E}+00$ & $0.0 \mathrm{E}+00$ & $0.0 \mathrm{E}+00$ \\
1080 & $0.0 \mathrm{E}+00$ & $0.0 \mathrm{E}+00$ & $0.0 \mathrm{E}+00$ \\
1120 & $0.0 \mathrm{E}+00$ & $0.0 \mathrm{E}+00$ & $0.0 \mathrm{E}+00$ \\
1175 & $0.0 \mathrm{E}+00$ & $0.0 \mathrm{E}+00$ & $0.0 \mathrm{E}+00$ \\
1575 & $0.0 \mathrm{E}+00$ & $0.0 \mathrm{E}+00$ & $0.0 \mathrm{E}+00$ \\
1750 & $0.0 \mathrm{E}+00$ & $0.0 \mathrm{E}+00$ & $0.0 \mathrm{E}+00$ \\
2175 & $0.0 \mathrm{E}+00$ & $0.0 \mathrm{E}+00$ & $0.0 \mathrm{E}+00$ \\
4000 & $0.0 \mathrm{E}+00$ & $0.0 \mathrm{E}+00$ & $0.0 \mathrm{E}+00$ \\
6000 & $6.8 \mathrm{E}+00$ & $0.0 \mathrm{E}+00$ & $0.0 \mathrm{E}+00$ \\
8000 & $4.1 \mathrm{E}+01$ & $0.0 \mathrm{E}+00$ & $0.0 \mathrm{E}+00$ \\
10000 & $4.1 \mathrm{E}+01$ & $0.0 \mathrm{E}+00$ & $0.0 \mathrm{E}+00$ \\
& & & \\
\hline & & &
\end{tabular}

\begin{abstract}
ABTDU Total Dose in Zone B Due to Atmospheric Release (SV $\left.\mathrm{Yr}^{-1}\right)$
\end{abstract}

\begin{tabular}{rcccccc}
\hline Time (yr) & U-238\&4 & Th-230 & Ra-226 & Pb-210 & Po-210 & Chain \\
\hline 1 & $1.2 \mathrm{E}-07$ & $1.7 \mathrm{E}-06$ & $3.0 \mathrm{E}-07$ & $1.5 \mathrm{E}-06$ & $1.8 \mathrm{E}-05$ & $2.1 \mathrm{E}-05$ \\
20 & $1.3 \mathrm{E}-07$ & $1.7 \mathrm{E}-06$ & $5.2 \mathrm{E}-07$ & $1.5 \mathrm{E}-06$ & $1.9 \mathrm{E}-05$ & $2.2 \mathrm{E}-05$ \\
25 & $1.3 \mathrm{E}-07$ & $1.7 \mathrm{E}-06$ & $5.7 \mathrm{E}-07$ & $1.5 \mathrm{E}-06$ & $1.9 \mathrm{E}-05$ & $2.3 \mathrm{E}-05$ \\
30 & $1.3 \mathrm{E}-07$ & $1.7 \mathrm{E}-06$ & $6.3 \mathrm{E}-07$ & $1.5 \mathrm{E}-06$ & $1.9 \mathrm{E}-05$ & $2.3 \mathrm{E}-05$ \\
62 & $1.3 \mathrm{E}-07$ & $1.7 \mathrm{E}-06$ & $9.2 \mathrm{E}-07$ & $1.5 \mathrm{E}-06$ & $2.0 \mathrm{E}-05$ & $2.4 \mathrm{E}-05$ \\
125 & $1.3 \mathrm{E}-07$ & $1.8 \mathrm{E}-06$ & $1.3 \mathrm{E}-06$ & $1.6 \mathrm{E}-06$ & $2.2 \mathrm{E}-05$ & $2.7 \mathrm{E}-05$ \\
575 & $1.3 \mathrm{E}-07$ & $1.7 \mathrm{E}-06$ & $2.1 \mathrm{E}-06$ & $1.6 \mathrm{E}-06$ & $2.5 \mathrm{E}-05$ & $3.0 \mathrm{E}-05$ \\
750 & $1.3 \mathrm{E}-07$ & $1.7 \mathrm{E}-06$ & $2.1 \mathrm{E}-06$ & $1.6 \mathrm{E}-06$ & $2.5 \mathrm{E}-05$ & $3.0 \mathrm{E}-05$ \\
1000 & $1.3 \mathrm{E}-07$ & $1.7 \mathrm{E}-06$ & $2.1 \mathrm{E}-06$ & $1.6 \mathrm{E}-06$ & $2.5 \mathrm{E}-05$ & $3.0 \mathrm{E}-05$ \\
1025 & $3.7 \mathrm{E}-09$ & $3.8 \mathrm{E}-09$ & $1.5 \mathrm{E}-06$ & $7.3 \mathrm{E}-08$ & $5.9 \mathrm{E}-06$ & $7.5 \mathrm{E}-06$ \\
1050 & $3.1 \mathrm{E}-09$ & $3.2 \mathrm{E}-09$ & $1.3 \mathrm{E}-06$ & $6.1 \mathrm{E}-08$ & $5.0 \mathrm{E}-06$ & $6.3 \mathrm{E}-06$ \\
1100 & $2.2 \mathrm{E}-09$ & $2.3 \mathrm{E}-09$ & $9.1 \mathrm{E}-07$ & $4.3 \mathrm{E}-08$ & $3.5 \mathrm{E}-06$ & $4.5 \mathrm{E}-06$ \\
1200 & $1.1 \mathrm{E}-09$ & $1.1 \mathrm{E}-09$ & $4.6 \mathrm{E}-07$ & $2.2 \mathrm{E}-08$ & $1.8 \mathrm{E}-06$ & $2.2 \mathrm{E}-06$ \\
1400 & $2.7 \mathrm{E}-10$ & $2.8 \mathrm{E}-10$ & $1.1 \mathrm{E}-07$ & $5.4 \mathrm{E}-09$ & $4.4 \mathrm{E}-07$ & $5.6 \mathrm{E}-07$ \\
1600 & $6.8 \mathrm{E}-11$ & $7.0 \mathrm{E}-11$ & $2.8 \mathrm{E}-08$ & $1.4 \mathrm{E}-09$ & $1.1 \mathrm{E}-07$ & $1.4 \mathrm{E}-07$ \\
2000 & $4.3 \mathrm{E}-12$ & $4.4 \mathrm{E}-12$ & $1.8 \mathrm{E}-09$ & $8.5 \mathrm{E}-11$ & $6.9 \mathrm{E}-09$ & $8.7 \mathrm{E}-09$ \\
2500 & $1.3 \mathrm{E}-13$ & $1.4 \mathrm{E}-13$ & $5.5 \mathrm{E}-11$ & $2.6 \mathrm{E}-12$ & $2.1 \mathrm{E}-10$ & $2.7 \mathrm{E}-10$ \\
3000 & $4.2 \mathrm{E}-15$ & $4.2 \mathrm{E}-15$ & $1.7 \mathrm{E}-12$ & $8.2 \mathrm{E}-14$ & $6.7 \mathrm{E}-12$ & $8.5 \mathrm{E}-12$ \\
\hline
\end{tabular}




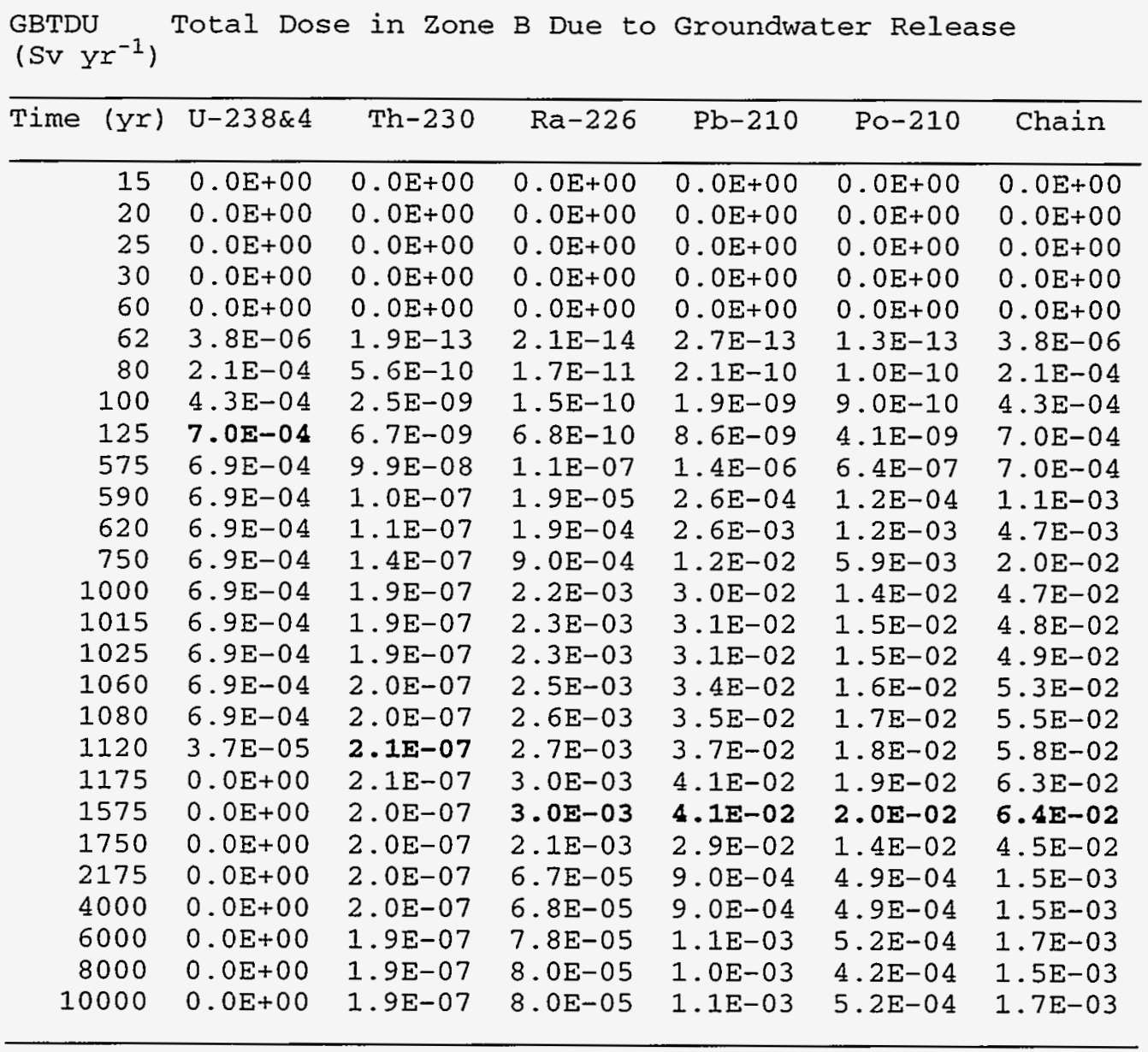

ABTIM Total Intake in Zone $B$ Due to Atmospheric Release (mg $\mathrm{yr}^{-1}$ )

\begin{tabular}{rrrr}
\hline Time $(y r)$ & As & Cr & Ni \\
\hline 1 & $2.0 E-01$ & $1.1 \mathrm{E}-03$ & $9.3 \mathrm{E}-05$ \\
20 & $2.1 \mathrm{E}-01$ & $1.2 \mathrm{E}-03$ & $9.5 \mathrm{E}-05$ \\
25 & $2.1 \mathrm{E}-01$ & $1.2 \mathrm{E}-03$ & $9.5 \mathrm{E}-05$ \\
30 & $2.2 \mathrm{E}-01$ & $1.2 \mathrm{E}-03$ & $9.6 \mathrm{E}-05$ \\
62 & $2.3 \mathrm{E}-01$ & $1.2 \mathrm{E}-03$ & $9.8 \mathrm{E}-05$ \\
125 & $2.5 \mathrm{E}-01$ & $1.3 \mathrm{E}-03$ & $1.0 \mathrm{E}-04$ \\
575 & $2.8 \mathrm{E}-01$ & $1.4 \mathrm{E}-03$ & $1.1 \mathrm{E}-04$ \\
750 & $2.8 \mathrm{E}-01$ & $1.4 \mathrm{E}-03$ & $1.1 \mathrm{E}-04$ \\
1000 & $2.8 \mathrm{E}-01$ & $1.4 \mathrm{E}-03$ & $1.1 \mathrm{E}-04$ \\
1025 & $6.4 \mathrm{E}-02$ & $2.7 \mathrm{E}-04$ & $1.1 \mathrm{E}-05$ \\
1050 & $5.4 \mathrm{E}-02$ & $2.3 \mathrm{E}-04$ & $9.5 \mathrm{E}-06$ \\
1100 & $3.8 \mathrm{E}-02$ & $1.6 \mathrm{E}-04$ & $6.7 \mathrm{E}-06$ \\
1200 & $1.9 \mathrm{E}-02$ & $8.1 \mathrm{E}-05$ & $3.4 \mathrm{E}-06$ \\
1400 & $4.7 \mathrm{E}-03$ & $2.0 \mathrm{E}-05$ & $8.4 \mathrm{E}-07$ \\
1600 & $1.2 \mathrm{E}-03$ & $5.0 \mathrm{E}-06$ & $2.1 \mathrm{E}-07$ \\
2000 & $7.4 \mathrm{E}-05$ & $3.1 \mathrm{E}-07$ & $1.3 \mathrm{E}-08$ \\
2500 & $2.3 \mathrm{E}-06$ & $9.8 \mathrm{E}-09$ & $4.1 \mathrm{E}-10$ \\
3000 & $7.2 \mathrm{E}-08$ & $3.1 \mathrm{E}-10$ & $1.3 \mathrm{E}-11$
\end{tabular}


GBTIM Total Intake in Zone B Due to Groundwater Release ( $\mathrm{mg} \mathrm{yr}^{-1}$ )

\begin{tabular}{rrrr}
\hline Time $(Y r)$ & As & $\mathrm{Cr}$ & $\mathrm{Ni}$ \\
\hline 15 & $0.0 \mathrm{E}+00$ & $5.3 \mathrm{E}-03$ & $3.0 \mathrm{E}-04$ \\
20 & $0.0 \mathrm{E}+00$ & $4.2 \mathrm{E}+02$ & $2.4 \mathrm{E}+01$ \\
25 & $0.0 \mathrm{E}+00$ & $8.4 \mathrm{E}+02$ & $4.7 \mathrm{E}+01$ \\
30 & $0.0 \mathrm{E}+00$ & $1.3 \mathrm{E}+03$ & $7.1 \mathrm{E}+01$ \\
60 & $0.0 \mathrm{E}+00$ & $1.3 \mathrm{E}+03$ & $7.1 \mathrm{E}+01$ \\
62 & $0.0 \mathrm{E}+00$ & $1.3 \mathrm{E}+03$ & $7.1 \mathrm{E}+01$ \\
80 & $0.0 \mathrm{E}+00$ & $1.3 \mathrm{E}+03$ & $7.1 \mathrm{E}+01$ \\
100 & $0.0 \mathrm{E}+00$ & $1.3 \mathrm{E}+03$ & $7.1 \mathrm{E}+01$ \\
125 & $0.0 \mathrm{E}+00$ & $1.3 \mathrm{E}+03$ & $7.1 \mathrm{E}+01$ \\
575 & $0.0 \mathrm{E}+00$ & $1.3 \mathrm{E}+03$ & $7.1 \mathrm{E}+01$ \\
590 & $0.0 \mathrm{E}+00$ & $1.2 \mathrm{E}+03$ & $7.1 \mathrm{E}+01$ \\
620 & $0.0 \mathrm{E}+00$ & $1.2 \mathrm{E}+03$ & $7.1 \mathrm{E}+01$ \\
750 & $0.0 \mathrm{E}+00$ & $1.2 \mathrm{E}+03$ & $7.1 \mathrm{E}+01$ \\
1000 & $0.0 \mathrm{E}+00$ & $1.2 \mathrm{E}+03$ & $7.1 \mathrm{E}+01$ \\
1015 & $0.0 \mathrm{E}+00$ & $1.2 \mathrm{E}+03$ & $7.1 \mathrm{E}+01$ \\
1025 & $0.0 \mathrm{E}+00$ & $4.2 \mathrm{E}+02$ & $2.4 \mathrm{E}+01$ \\
1060 & $0.0 \mathrm{E}+00$ & $0.0 \mathrm{E}+00$ & $0.0 \mathrm{E}+00$ \\
1080 & $0.0 \mathrm{E}+00$ & $0.0 \mathrm{E}+00$ & $0.0 \mathrm{E}+00$ \\
1120 & $0.0 \mathrm{E}+00$ & $0.0 \mathrm{E}+00$ & $0.0 \mathrm{E}+00$ \\
1175 & $0.0 \mathrm{E}+00$ & $0.0 \mathrm{E}+00$ & $0.0 \mathrm{E}+00$ \\
1575 & $0.0 \mathrm{E}+00$ & $0.0 \mathrm{E}+00$ & $0.0 \mathrm{E}+00$ \\
1750 & $0.0 \mathrm{E}+00$ & $0.0 \mathrm{E}+00$ & $0.0 \mathrm{E}+00$ \\
2175 & $0.0 \mathrm{E}+00$ & $0.0 \mathrm{E}+00$ & $0.0 \mathrm{E}+00$ \\
4000 & $0.0 \mathrm{E}+00$ & $0.0 \mathrm{E}+00$ & $0.0 \mathrm{E}+00$ \\
6000 & $8.9 \mathrm{E}-01$ & $0.0 \mathrm{E}+00$ & $0.0 \mathrm{E}+00$ \\
8000 & $5.5 \mathrm{E}+00$ & $0.0 \mathrm{E}+00$ & $0.0 \mathrm{E}+00$ \\
10000 & $5.5 \mathrm{E}+00$ & $0.0 \mathrm{E}+00$ & $0.0 \mathrm{E}+00$
\end{tabular}

AATRU Total Cancer Risk in Zone A Due to Atmospheric Release

\begin{tabular}{rcccccc}
\hline Time (yr) & $\mathrm{U}-238 \& 4$ & Th-230 & Ra-226 & Pb-210 & Po-210 & Chain \\
\hline 1 & $1.4 \mathrm{E}-06$ & $8.4 \mathrm{E}-06$ & $2.3 \mathrm{E}-06$ & $4.6 \mathrm{E}-05$ & $6.5 \mathrm{E}-06$ & $6.4 \mathrm{E}-05$ \\
20 & $1.5 \mathrm{E}-06$ & $8.4 \mathrm{E}-06$ & $5.3 \mathrm{E}-06$ & $4.6 \mathrm{E}-05$ & $8.7 \mathrm{E}-06$ & $6.9 \mathrm{E}-05$ \\
25 & $1.5 \mathrm{E}-06$ & $8.4 \mathrm{E}-06$ & $6.0 \mathrm{E}-06$ & $4.6 \mathrm{E}-05$ & $9.2 \mathrm{E}-06$ & $7.1 \mathrm{E}-05$ \\
30 & $1.5 \mathrm{E}-06$ & $8.4 \mathrm{E}-06$ & $6.7 \mathrm{E}-06$ & $4.6 \mathrm{E}-05$ & $9.8 \mathrm{E}-06$ & $7.2 \mathrm{E}-05$ \\
62 & $1.5 \mathrm{E}-06$ & $8.4 \mathrm{E}-06$ & $1.1 \mathrm{E}-05$ & $4.6 \mathrm{E}-05$ & $1.3 \mathrm{E}-05$ & $7.9 \mathrm{E}-05$ \\
125 & $1.5 \mathrm{E}-06$ & $8.3 \mathrm{E}-06$ & $1.6 \mathrm{E}-05$ & $4.6 \mathrm{E}-05$ & $1.7 \mathrm{E}-05$ & $8.9 \mathrm{E}-05$ \\
575 & $1.5 \mathrm{E}-06$ & $8.3 \mathrm{E}-06$ & $2.6 \mathrm{E}-05$ & $4.6 \mathrm{E}-05$ & $2.4 \mathrm{E}-05$ & $1.1 \mathrm{E}-04$ \\
750 & $1.5 \mathrm{E}-06$ & $8.3 \mathrm{E}-06$ & $2.6 \mathrm{E}-05$ & $4.6 \mathrm{E}-05$ & $2.4 \mathrm{E}-05$ & $1.1 \mathrm{E}-04$ \\
1000 & $1.5 \mathrm{E}-06$ & $8.3 \mathrm{E}-06$ & $2.6 \mathrm{E}-05$ & $4.6 \mathrm{E}-05$ & $2.4 \mathrm{E}-05$ & $1.1 \mathrm{E}-04$ \\
1025 & $2.4 \mathrm{E}-08$ & $1.6 \mathrm{E}-09$ & $2.0 \mathrm{E}-05$ & $3.0 \mathrm{E}-07$ & $1.5 \mathrm{E}-05$ & $3.6 \mathrm{E}-05$ \\
1050 & $2.1 \mathrm{E}-08$ & $1.3 \mathrm{E}-09$ & $1.7 \mathrm{E}-05$ & $2.5 \mathrm{E}-07$ & $1.3 \mathrm{E}-05$ & $3.0 \mathrm{E}-05$ \\
1100 & $1.5 \mathrm{E}-08$ & $9.5 \mathrm{E}-10$ & $1.2 \mathrm{E}-05$ & $1.8 \mathrm{E}-07$ & $9.0 \mathrm{E}-06$ & $2.1 \mathrm{E}-05$ \\
1200 & $7.3 \mathrm{E}-09$ & $4.7 \mathrm{E}-10$ & $6.1 \mathrm{E}-06$ & $9.0 \mathrm{E}-08$ & $4.5 \mathrm{E}-06$ & $1.1 \mathrm{E}-05$ \\
1400 & $1.8 \mathrm{E}-09$ & $1.2 \mathrm{E}-10$ & $1.5 \mathrm{E}-06$ & $2.2 \mathrm{E}-08$ & $1.1 \mathrm{E}-06$ & $2.7 \mathrm{E}-06$ \\
1600 & $4.5 \mathrm{E}-10$ & $3.0 \mathrm{E}-11$ & $3.8 \mathrm{E}-07$ & $5.6 \mathrm{E}-09$ & $2.8 \mathrm{E}-07$ & $6.7 \mathrm{E}-07$ \\
2000 & $2.8 \mathrm{E}-11$ & $1.8 \mathrm{E}-12$ & $2.4 \mathrm{E}-08$ & $3.5 \mathrm{E}-10$ & $1.8 \mathrm{E}-08$ & $4.2 \mathrm{E}-08$ \\
2500 & $8.9 \mathrm{E}-13$ & $5.7 \mathrm{E}-14$ & $7.4 \mathrm{E}-10$ & $1.1 \mathrm{E}-11$ & $5.5 \mathrm{E}-10$ & $1.3 \mathrm{E}-09$ \\
3000 & $2.8 \mathrm{E}-14$ & $1.8 \mathrm{E}-15$ & $2.3 \mathrm{E}-11$ & $3.4 \mathrm{E}-13$ & $1.7 \mathrm{E}-11$ & $4.0 \mathrm{E}-11$ \\
\hline
\end{tabular}


GATRU Total Cancer Risk in zone A Due to Groundwater Release

\begin{tabular}{rllllll}
\hline Time $(Y r)$ & $\mathrm{U}-238 \& 4$ & $\mathrm{Th}-230$ & $\mathrm{Ra}-226$ & $\mathrm{~Pb}-210$ & Po-210 & Chain \\
\hline 15 & $0.0 \mathrm{E}+00$ & $0.0 \mathrm{E}+00$ & $0.0 \mathrm{E}+00$ & $0.0 \mathrm{E}+00$ & $0.0 \mathrm{E}+00$ & $0.0 \mathrm{E}+00$ \\
20 & $0.0 \mathrm{E}+00$ & $0.0 \mathrm{E}+00$ & $0.0 \mathrm{E}+00$ & $0.0 \mathrm{E}+00$ & $0.0 \mathrm{E}+00$ & $0.0 \mathrm{E}+00$ \\
25 & $0.0 \mathrm{E}+00$ & $0.0 \mathrm{E}+00$ & $0.0 \mathrm{E}+00$ & $0.0 \mathrm{E}+00$ & $0.0 \mathrm{E}+00$ & $0.0 \mathrm{E}+00$ \\
30 & $0.0 \mathrm{E}+00$ & $0.0 \mathrm{E}+00$ & $0.0 \mathrm{E}+00$ & $0.0 \mathrm{E}+00$ & $0.0 \mathrm{E}+00$ & $0.0 \mathrm{E}+00$ \\
60 & $0.0 \mathrm{E}+00$ & $0.0 \mathrm{E}+00$ & $0.0 \mathrm{E}+00$ & $0.0 \mathrm{E}+00$ & $0.0 \mathrm{E}+00$ & $0.0 \mathrm{E}+00$ \\
62 & $3.1 \mathrm{E}-05$ & $7.0 \mathrm{E}-14$ & $1.4 \mathrm{E}-13$ & $7.0 \mathrm{E}-13$ & $4.5 \mathrm{E}-13$ & $3.1 \mathrm{E}-05$ \\
80 & $1.7 \mathrm{E}-03$ & $2.1 \mathrm{E}-10$ & $1.1 \mathrm{E}-10$ & $5.6 \mathrm{E}-10$ & $3.6 \mathrm{E}-10$ & $1.7 \mathrm{E}-03$ \\
100 & $3.5 \mathrm{E}-03$ & $9.3 \mathrm{E}-10$ & $1.0 \mathrm{E}-09$ & $4.9 \mathrm{E}-09$ & $3.2 \mathrm{E}-09$ & $3.5 \mathrm{E}-03$ \\
125 & $5.7 \mathrm{E}-03$ & $2.5 \mathrm{E}-09$ & $4.5 \mathrm{E}-09$ & $2.2 \mathrm{E}-08$ & $1.4 \mathrm{E}-08$ & $5.7 \mathrm{E}-03$ \\
575 & $5.7 \mathrm{E}-03$ & $3.8 \mathrm{E}-08$ & $7.1 \mathrm{E}-07$ & $3.5 \mathrm{E}-06$ & $2.3 \mathrm{E}-06$ & $5.7 \mathrm{E}-03$ \\
590 & $5.7 \mathrm{E}-03$ & $3.9 \mathrm{E}-08$ & $1.3 \mathrm{E}-04$ & $6.8 \mathrm{E}-04$ & $4.4 \mathrm{E}-04$ & $6.9 \mathrm{E}-03$ \\
620 & $5.7 \mathrm{E}-03$ & $4.1 \mathrm{E}-08$ & $1.3 \mathrm{E}-03$ & $6.7 \mathrm{E}-03$ & $4.3 \mathrm{E}-03$ & $1.8 \mathrm{E}-02$ \\
750 & $5.7 \mathrm{E}-03$ & $5.1 \mathrm{E}-08$ & $6.0 \mathrm{E}-03$ & $3.2 \mathrm{E}-02$ & $2.1 \mathrm{E}-02$ & $6.4 \mathrm{E}-02$ \\
1000 & $5.7 \mathrm{E}-03$ & $7.1 \mathrm{E}-08$ & $1.4 \mathrm{E}-02$ & $7.7 \mathrm{E}-02$ & $4.9 \mathrm{E}-02$ & $1.5 \mathrm{E}-01$ \\
1015 & $5.7 \mathrm{E}-03$ & $7.2 \mathrm{E}-08$ & $1.5 \mathrm{E}-02$ & $7.9 \mathrm{E}-02$ & $5.1 \mathrm{E}-02$ & $1.5 \mathrm{E}-01$ \\
1025 & $5.7 \mathrm{E}-03$ & $7.3 \mathrm{E}-08$ & $1.5 \mathrm{E}-02$ & $8.1 \mathrm{E}-02$ & $5.2 \mathrm{E}-02$ & $1.5 \mathrm{E}-01$ \\
1060 & $5.7 \mathrm{E}-03$ & $7.6 \mathrm{E}-08$ & $1.6 \mathrm{E}-02$ & $8.7 \mathrm{E}-02$ & $5.6 \mathrm{E}-02$ & $1.7 \mathrm{E}-01$ \\
1080 & $5.7 \mathrm{E}-03$ & $7.8 \mathrm{E}-08$ & $1.7 \mathrm{E}-02$ & $9.0 \mathrm{E}-02$ & $5.8 \mathrm{E}-02$ & $1.7 \mathrm{E}-01$ \\
1120 & $3.1 \mathrm{E}-04$ & $7.8 \mathrm{E}-08$ & $1.8 \mathrm{E}-02$ & $9.7 \mathrm{E}-02$ & $6.2 \mathrm{E}-02$ & $1.8 \mathrm{E}-01$ \\
1175 & $1.1 \mathrm{E}-06$ & $7.8 \mathrm{E}-08$ & $2.0 \mathrm{E}-02$ & $1.1 \mathrm{E}-01$ & $6.8 \mathrm{E}-02$ & $1.9 \mathrm{E}-01$ \\
1575 & $2.0 \mathrm{E}-06$ & $7.8 \mathrm{E}-08$ & $2.0 \mathrm{E}-02$ & $1.1 \mathrm{E}-01$ & $6.8 \mathrm{E}-02$ & $1.9 \mathrm{E}-01$ \\
1750 & $1.0 \mathrm{E}-09$ & $7.7 \mathrm{E}-08$ & $1.4 \mathrm{E}-02$ & $7.5 \mathrm{E}-02$ & $4.8 \mathrm{E}-02$ & $1.4 \mathrm{E}-01$ \\
2175 & $1.1 \mathrm{E}-06$ & $7.6 \mathrm{E}-08$ & $4.6 \mathrm{E}-04$ & $2.5 \mathrm{E}-03$ & $1.6 \mathrm{E}-03$ & $4.5 \mathrm{E}-03$ \\
4000 & $2.1 \mathrm{E}-06$ & $7.4 \mathrm{E}-08$ & $4.6 \mathrm{E}-04$ & $2.5 \mathrm{E}-03$ & $1.6 \mathrm{E}-03$ & $4.6 \mathrm{E}-03$ \\
6000 & $1.0 \mathrm{E}-07$ & $7.3 \mathrm{E}-08$ & $4.7 \mathrm{E}-04$ & $2.8 \mathrm{E}-03$ & $1.8 \mathrm{E}-03$ & $5.1 \mathrm{E}-03$ \\
8000 & $1.0 \mathrm{E}-06$ & $7.2 \mathrm{E}-08$ & $5.0 \mathrm{E}-04$ & $2.7 \mathrm{E}-03$ & $1.7 \mathrm{E}-03$ & $4.9 \mathrm{E}-03$ \\
10000 & $1.1 \mathrm{E}-06$ & $7.0 \mathrm{E}-08$ & $5.3 \mathrm{E}-04$ & $2.8 \mathrm{E}-03$ & $1.8 \mathrm{E}-03$ & $5.1 \mathrm{E}-03$ \\
& & & & & & \\
\hline
\end{tabular}

AATRM Total Cancer Risk in

Zone A Due to Atmospheric Release

\begin{tabular}{rrrr}
\hline Time (yr) & As & Cr & Ni \\
\hline 1 & $2.0 \mathrm{E}-02$ & $4.2 \mathrm{E}-05$ & $9.0 \mathrm{E}-08$ \\
20 & $2.1 \mathrm{E}-02$ & $4.2 \mathrm{E}-05$ & $9.0 \mathrm{E}-08$ \\
25 & $2.1 \mathrm{E}-02$ & $4.2 \mathrm{E}-05$ & $9.0 \mathrm{E}-08$ \\
30 & $2.1 \mathrm{E}-02$ & $4.2 \mathrm{E}-05$ & $9.0 \mathrm{E}-08$ \\
62 & $2.3 \mathrm{E}-02$ & $4.2 \mathrm{E}-05$ & $9.0 \mathrm{E}-08$ \\
125 & $2.4 \mathrm{E}-02$ & $4.2 \mathrm{E}-05$ & $9.0 \mathrm{E}-08$ \\
575 & $2.7 \mathrm{E}-02$ & $4.2 \mathrm{E}-05$ & $9.0 \mathrm{E}-08$ \\
750 & $2.7 \mathrm{E}-02$ & $4.2 \mathrm{E}-05$ & $9.0 \mathrm{E}-08$ \\
1000 & $2.7 \mathrm{E}-02$ & $4.2 \mathrm{E}-05$ & $9.0 \mathrm{E}-08$ \\
1025 & $6.2 \mathrm{E}-03$ & $0.0 \mathrm{E}+00$ & $0.0 \mathrm{E}+00$ \\
1050 & $5.2 \mathrm{E}-03$ & $0.0 \mathrm{E}+00$ & $0.0 \mathrm{E}+00$ \\
1100 & $3.7 \mathrm{E}-03$ & $0.0 \mathrm{E}+00$ & $0.0 \mathrm{E}+00$ \\
1200 & $1.8 \mathrm{E}-03$ & $0.0 \mathrm{E}+00$ & $0.0 \mathrm{E}+00$ \\
1400 & $4.6 \mathrm{E}-04$ & $0.0 \mathrm{E}+00$ & $0.0 \mathrm{E}+00$ \\
1600 & $1.1 \mathrm{E}-04$ & $0.0 \mathrm{E}+00$ & $0.0 \mathrm{E}+00$ \\
2000 & $7.2 \mathrm{E}-06$ & $0.0 \mathrm{E}+00$ & $0.0 \mathrm{E}+00$ \\
2500 & $2.2 \mathrm{E}-07$ & $0.0 \mathrm{E}+00$ & $0.0 \mathrm{E}+00$ \\
3000 & $7.0 \mathrm{E}-09$ & $0.0 \mathrm{E}+00$ & $0.0 \mathrm{E}+00$
\end{tabular}


GATRM Total Cancer in Zone $A$ Due to Groundwater Release

\begin{tabular}{|c|c|c|c|}
\hline Time (yr) & As & $\mathrm{Cr}$ & $\mathrm{Ni}$ \\
\hline 15 & $0.0 E+00$ & $0.0 E+00$ & $0.0 \mathrm{E}+00$ \\
\hline 20 & $0.0 \mathrm{E}+00$ & $0.0 E+00$ & $0.0 \mathrm{E}+00$ \\
\hline 25 & $0.0 \mathrm{E}+00$ & $0.0 E+00$ & $0.0 E+00$ \\
\hline 30 & $0.0 \mathrm{E}+00$ & $0.0 E+00$ & $0.0 \mathrm{E}+00$ \\
\hline 60 & $0.0 \mathrm{E}+00$ & $0.0 \mathrm{E}+00$ & $0.0 \mathrm{E}+00$ \\
\hline 62 & $0.0 E+00$ & $0.0 E+00$ & $0.0 \mathrm{E}+00$ \\
\hline 80 & $0.0 \mathrm{E}+00$ & $0.0 \mathrm{E}+00$ & $0.0 \mathrm{E}+00$ \\
\hline 100 & $0.0 E+00$ & $0.0 E+00$ & $0.0 \mathrm{E}+00$ \\
\hline 125 & $0.0 E+00$ & $0.0 E+00$ & $0.0 \mathrm{E}+00$ \\
\hline 575 & $0.0 E+00$ & $0.0 E+00$ & $0.0 \mathrm{E}+00$ \\
\hline 590 & $0.0 E+00$ & $0.0 E+00$ & $0.0 \mathrm{E} \div 00$ \\
\hline 620 & $0.0 E+00$ & $0.0 \mathrm{E}+00$ & $0.0 \mathrm{E} \div 00$ \\
\hline 750 & $0.0 E+00$ & $0.0 E+00$ & $0.0 \mathrm{E}+00$ \\
\hline 1000 & $0.0 \mathrm{E}+00$ & $0.0 \mathrm{E}+00$ & $0.0 \mathrm{E}+00$ \\
\hline 1015 & $0.0 \mathrm{E}+00$ & $0.0 \mathrm{E}+00$ & $0.0 \mathrm{E}+00$ \\
\hline 1025 & $0.0 \mathrm{E}+00$ & $0.0 E+00$ & $0.0 \mathrm{E}+00$ \\
\hline 1060 & $0.0 E+00$ & $0.0 E+00$ & $0.0 \mathrm{E}+00$ \\
\hline 1080 & $0.0 \mathrm{E}+00$ & $0.0 E+00$ & $0.0 \mathrm{E}+00$ \\
\hline 1120 & $0.0 \mathrm{E}+00$ & $0.0 E+00$ & $0.0 E+00$ \\
\hline 1175 & $0.0 E+00$ & $0.0 E \div 00$ & $0.0 E+00$ \\
\hline 1575 & $0.0 E+00$ & $0.0 E+00$ & $0.0 \mathrm{E}+00$ \\
\hline 1750 & $0.0 \mathrm{E}+00$ & $0.0 E+00$ & $0.0 \mathrm{E}+00$ \\
\hline 2175 & $0.0 \mathrm{E}+00$ & $0.0 \mathrm{E}+00$ & $0.0 \mathrm{E}+00$ \\
\hline 4000 & $0.0 E+00$ & $0.0 \mathrm{E}+00$ & $0.0 \mathrm{E}+00$ \\
\hline 6000 & 1. $2 \mathrm{E}-01$ & $0.0 E+00$ & $0.0 \mathrm{E}+00$ \\
\hline 8000 & $7.2 \mathrm{E}-01$ & $0.0 \mathrm{E}+00$ & $0.0 \mathrm{E}+00$ \\
\hline 10000 & $7.2 E-01$ & $0.0 E+00$ & $0.0 E+00$ \\
\hline
\end{tabular}

ABTRU Total Cancer Risk in Zone B Due to Atmospheric Release

\begin{tabular}{rrrrrrr}
\hline Time (yr) & $\mathrm{U}-238 \& 4$ & Th-230 & Ra-226 & Pb-210 & Po-210 & Chain \\
\hline 1 & $2.6 \mathrm{E}-07$ & $1.5 \mathrm{E}-06$ & $4.2 \mathrm{E}-07$ & $8.3 \mathrm{E}-06$ & $1.2 \mathrm{E}-06$ & $1.2 \mathrm{E}-05$ \\
25 & $2.6 \mathrm{E}-07$ & $1.5 \mathrm{E}-06$ & $9.6 \mathrm{E}-07$ & $8.3 \mathrm{E}-06$ & $1.6 \mathrm{E}-06$ & $1.3 \mathrm{E}-05$ \\
30 & $2.6 \mathrm{E}-07$ & $1.5 \mathrm{E}-06$ & $1.1 \mathrm{E}-06$ & $8.3 \mathrm{E}-06$ & $1.7 \mathrm{E}-06$ & $1.3 \mathrm{E}-05$ \\
62 & $2.6 \mathrm{E}-07$ & $1.5 \mathrm{E}-06$ & $1.2 \mathrm{E}-06$ & $8.3 \mathrm{E}-06$ & $1.8 \mathrm{E}-06$ & $1.3 \mathrm{E}-05$ \\
125 & $2.7 \mathrm{E}-07$ & $1.5 \mathrm{E}-06$ & $2.9 \mathrm{E}-06$ & $8.3 \mathrm{E}-06$ & $3.1 \mathrm{E}-06$ & $1.6 \mathrm{E}-05$ \\
575 & $2.7 \mathrm{E}-07$ & $1.5 \mathrm{E}-06$ & $4.7 \mathrm{E}-06$ & $8.3 \mathrm{E}-06$ & $4.4 \mathrm{E}-06$ & $1.9 \mathrm{E}-05$ \\
750 & $2.7 \mathrm{E}-07$ & $1.5 \mathrm{E}-06$ & $4.8 \mathrm{E}-06$ & $8.3 \mathrm{E}-06$ & $4.4 \mathrm{E}-06$ & $1.9 \mathrm{E}-05$ \\
1000 & $2.7 \mathrm{E}-07$ & $1.5 \mathrm{E}-06$ & $4.8 \mathrm{E}-06$ & $8.3 \mathrm{E}-06$ & $4.4 \mathrm{E}-06$ & $1.9 \mathrm{E}-05$ \\
1025 & $4.4 \mathrm{E}-09$ & $2.9 \mathrm{E}-10$ & $3.7 \mathrm{E}-06$ & $5.5 \mathrm{E}-08$ & $2.7 \mathrm{E}-06$ & $6.5 \mathrm{E}-06$ \\
1050 & $3.7 \mathrm{E}-09$ & $2.4 \mathrm{E}-10$ & $3.1 \mathrm{E}-06$ & $4.6 \mathrm{E}-08$ & $2.3 \mathrm{E}-06$ & $5.5 \mathrm{E}-06$ \\
1100 & $2.6 \mathrm{E}-09$ & $1.7 \mathrm{E}-10$ & $2.2 \mathrm{E}-06$ & $3.3 \mathrm{E}-08$ & $1.6 \mathrm{E}-06$ & $3.9 \mathrm{E}-06$ \\
1200 & $1.3 \mathrm{E}-09$ & $8.6 \mathrm{E}-11$ & $1.1 \mathrm{E}-06$ & $1.6 \mathrm{E}-08$ & $8.2 \mathrm{E}-07$ & $1.9 \mathrm{E}-06$ \\
1400 & $3.3 \mathrm{E}-10$ & $2.1 \mathrm{E}-11$ & $2.8 \mathrm{E}-07$ & $4.1 \mathrm{E}-09$ & $2.0 \mathrm{E}-07$ & $4.8 \mathrm{E}-07$ \\
1600 & $8.2 \mathrm{E}-11$ & $5.4 \mathrm{E}-12$ & $6.9 \mathrm{E}-08$ & $1.0 \mathrm{E}-09$ & $5.1 \mathrm{E}-08$ & $1.2 \mathrm{E}-07$ \\
2000 & $5.1 \mathrm{E}-12$ & $3.3 \mathrm{E}-13$ & $4.3 \mathrm{E}-09$ & $6.3 \mathrm{E}-11$ & $3.2 \mathrm{E}-09$ & $7.5 \mathrm{E}-09$ \\
2500 & $1.6 \mathrm{E}-13$ & $1.0 \mathrm{E}-14$ & $1.3 \mathrm{E}-10$ & $2.0 \mathrm{E}-12$ & $9.9 \mathrm{E}-11$ & $2.4 \mathrm{E}-10$ \\
3000 & $5.0 \mathrm{E}-15$ & $3.2 \mathrm{E}-16$ & $4.2 \mathrm{E}-12$ & $6.2 \mathrm{E}-14$ & $3.1 \mathrm{E}-12$ & $7.3 \mathrm{E}-12$ \\
& & & & & &
\end{tabular}


GBTRU Total Cancer Risk in Zone B Due to Groundwater Release

\begin{tabular}{|c|c|c|c|c|c|c|}
\hline Time (yr) & $U-238 \& 4$ & Th-230 & $\mathrm{Ra}-226$ & $\mathrm{~Pb}-210$ & Po-210 & Chain \\
\hline 15 & $0.0 \mathrm{E}+00$ & $0.0 \mathrm{E}+00$ & $0.0 \mathrm{E}+00$ & $0.0 E+00$ & $0.0 \mathrm{E}+00$ & $0.0 E+00$ \\
\hline 20 & $0.0 \mathrm{E}+00$ & $0.0 \mathrm{E}+00$ & $0.0 \mathrm{E}+00$ & $0.0 E+00$ & $0.0 \mathrm{E}+00$ & $0.0 \mathrm{E}+00$ \\
\hline 25 & $0.0 \mathrm{E}+00$ & $0.0 \mathrm{E}+00$ & $0.0 \mathrm{E}+00$ & $0.0 E+00$ & $0.0 \mathrm{E}+00$ & $0.0 \mathrm{E}+00$ \\
\hline 30 & $0.0 \mathrm{E}+00$ & $0.0 \mathrm{E}+00$ & $0.0 E+00$ & $0.0 E+00$ & $0.0 E+00$ & $0.0 \mathrm{E}+00$ \\
\hline 60 & $0.0 \mathrm{E}+00$ & $0.0 E+00$ & $0.0 E+00$ & $0.0 \mathrm{E}+00$ & $0.0 E+00$ & $0.0 \mathrm{E}+00$ \\
\hline 62 & $3.9 \mathrm{E}-06$ & 1. $3 E-14$ & 2. $2 E-14$ & 2. $0 \mathrm{E}-13$ & $5.9 \mathrm{E}-14$ & $3.9 E-06$ \\
\hline 80 & $2.1 \mathrm{E}-04$ & $3.9 E-11$ & $1.7 \mathrm{E}-11$ & 1. $6 \mathrm{E}-10$ & $4.7 \mathrm{E}-11$ & $2.1 E-04$ \\
\hline 100 & $4.4 \mathrm{E}-04$ & $1.7 \mathrm{E}-10$ & 1. $5 \mathrm{E}-10$ & $1.4 E-09$ & $4.2 \mathrm{E}-10$ & $4.4 E-04$ \\
\hline 125 & $7.1 E-04$ & $4.7 \mathrm{E}-10$ & $6.9 \mathrm{E}-10$ & $6.5 E-09$ & $1.9 \mathrm{E}-09$ & $7.1 \mathrm{E}-04$ \\
\hline 575 & 7.1E-04 & $7.0 \mathrm{E}-09$ & $1.1 \mathrm{E}-07$ & $1.0 \mathrm{E}-06$ & $3.0 E-07$ & $7.2 \mathrm{E}-04$ \\
\hline 590 & $7.1 \mathrm{E}-04$ & $7.2 \mathrm{E}-09$ & $2.0 \mathrm{E}-05$ & $2.0 \mathrm{E}-04$ & $5.8 E-05$ & $9.9 \mathrm{E}-04$ \\
\hline 620 & $7.1 \mathrm{E}-04$ & $7.6 \mathrm{E}-09$ & $1.9 E-04$ & $1.9 \mathrm{E}-03$ & $5.7 E-04$ & $3.4 \mathrm{E}-03$ \\
\hline 750 & $7.1 \mathrm{E}-04$ & $9.5 E-09$ & $9.2 E-04$ & $9.3 E-03$ & $2.7 E-03$ & $1.4 \mathrm{E}-02$ \\
\hline 1000 & $7.1 \mathrm{E}-04$ & $1.3 E-08$ & $2.2 \mathrm{E}-03$ & $2.2 \mathrm{E}-02$ & $6.5 E-03$ & $3.2 \mathrm{E}-02$ \\
\hline 1015 & $7.1 \mathrm{E}-04$ & $1.3 E-08$ & $2.3 E-03$ & $2.3 E-02$ & $6.8 E-03$ & $3.3 E-02$ \\
\hline 1025 & $7.1 E-04$ & $1.4 \mathrm{E}-08$ & $2.3 \mathrm{E}-03$ & $2.4 E-02$ & $6.9 \mathrm{E}-03$ & $3.4 \mathrm{E}-02$ \\
\hline 1060 & $7.1 E-04$ & $1.4 \mathrm{E}-08$ & $2.5 \mathrm{E}-03$ & $2.5 E-02$ & $7.4 \mathrm{E}-03$ & $3.6 E-02$ \\
\hline 1080 & $7.1 \mathrm{E}-04$ & $1.4 \mathrm{E}-08$ & $2.6 \mathrm{E}-03$ & $2.6 \mathrm{E}-02$ & $7.7 \mathrm{E}-03$ & $3.7 \mathrm{E}-02$ \\
\hline 1120 & $3.9 E-05$ & $1.5 \mathrm{E}-08$ & $2.8 E-03$ & $2.8 \mathrm{E}-02$ & $8.2 E-03$ & $3.9 \mathrm{E}-02$ \\
\hline 1175 & $0.0 \mathrm{E}+00$ & $1.5 E-08$ & $3.0 \mathrm{E}-03$ & $3.1 \mathrm{E}-02$ & $9.0 \mathrm{E}-03$ & $4.3 E-02$ \\
\hline 1575 & $0.0 \mathrm{E}+00$ & $1.4 \mathrm{E}-08$ & $3.1 \mathrm{E}-03$ & $3.1 \mathrm{E}-02$ & $9.0 \mathrm{E}-03$ & $4.3 E-12$ \\
\hline 1750 & $0.0 E+00$ & 1. $4 \mathrm{E}-08$ & $2.2 \mathrm{E}-03$ & $2.2 \mathrm{E}-02$ & $6.4 \mathrm{E}-03$ & $3.0 E-02$ \\
\hline 2175 & $0.0 E+00$ & $1.4 \mathrm{E}-08$ & $7.0 \mathrm{E}-05$ & $7.0 \mathrm{E}-04$ & $2.1 E-04$ & $9.8 E-04$ \\
\hline 4000 & $0.0 E+00$ & $1.4 \mathrm{E}-08$ & $7.2 \mathrm{E}-05$ & $7.2 \mathrm{E}-04$ & $2.1 E-04$ & $1.0 \mathrm{E}-03$ \\
\hline 6000 & $0.0 E+00$ & 1. $3 E-08$ & $7.3 \mathrm{E}-05$ & $8.0 \mathrm{E}-04$ & $2.4 \mathrm{E}-04$ & $1.1 \mathrm{E}-03$ \\
\hline 8000 & $0.0 E+00$ & 1. $3 \mathrm{E}-08$ & $7.7 E-05$ & $7.7 \mathrm{E}-04$ & $2.3 E-04$ & $1.1 \mathrm{E}-03$ \\
\hline 10000 & $0.0 \mathrm{E}+00$ & 1. $3 E-08$ & $8.1 E-05$ & $8.1 \mathrm{E}-04$ & $2.4 E-04$ & $1.1 \mathrm{E}-03$ \\
\hline
\end{tabular}

ABTRM Total cancer Risk in Zone B Due to Atmospheric Release

\begin{tabular}{rrrr}
\hline Time $(y r)$ & As & Cr & $N i$ \\
\hline 1 & $3.6 \mathrm{E}-03$ & $7.6 \mathrm{E}-06$ & $1.6 \mathrm{E}-08$ \\
20 & $3.8 \mathrm{E}-03$ & $7.6 \mathrm{E}-06$ & $1.6 \mathrm{E}-08$ \\
25 & $3.8 \mathrm{E}-03$ & $7.6 \mathrm{E}-06$ & $1.6 \mathrm{E}-08$ \\
30 & $3.9 \mathrm{E}-03$ & $7.6 \mathrm{E}-06$ & $1.6 \mathrm{E}-08$ \\
62 & $4.1 \mathrm{E}-03$ & $7.6 \mathrm{E}-06$ & $1.6 \mathrm{E}-08$ \\
125 & $4.4 \mathrm{E}-03$ & $7.6 \mathrm{E}-06$ & $1.6 \mathrm{E}-08$ \\
575 & $4.9 \mathrm{E}-03$ & $7.6 \mathrm{E}-06$ & $1.6 \mathrm{E}-08$ \\
750 & $5.0 \mathrm{E}-03$ & $7.6 \mathrm{E}-06$ & $1.6 \mathrm{E}-08$ \\
1000 & $5.0 \mathrm{E}-03$ & $7.6 \mathrm{E}-06$ & $1.6 \mathrm{E}-08$ \\
1025 & $1.1 \mathrm{E}-03$ & $0.0 \mathrm{E}+00$ & $0.0 \mathrm{E}+00$ \\
1050 & $9.4 \mathrm{E}-04$ & $0.0 \mathrm{E}+00$ & $0.0 \mathrm{E}+00$ \\
1100 & $6.6 \mathrm{E}-04$ & $0.0 \mathrm{E}+00$ & $0.0 \mathrm{E}+00$ \\
1200 & $3.3 \mathrm{E}-04$ & $0.0 \mathrm{E}+00$ & $0.0 \mathrm{E}+00$ \\
1400 & $8.3 \mathrm{E}-05$ & $0.0 \mathrm{E}+00$ & $0.0 \mathrm{E}+00$ \\
1600 & $2.1 \mathrm{E}-05$ & $0.0 \mathrm{E}+00$ & $0.0 \mathrm{E}+00$ \\
2000 & $1.3 \mathrm{E}-06$ & $0.0 \mathrm{E}+00$ & $0.0 \mathrm{E}+00$ \\
2500 & $4.0 \mathrm{E}-08$ & $0.0 \mathrm{E}+00$ & $0.0 \mathrm{E}+00$ \\
3000 & $1.3 \mathrm{E}-09$ & $0.0 \mathrm{E}+00$ & $0.0 \mathrm{E}+00$ \\
& & & \\
\hline
\end{tabular}


GBTRM Total Cancer in Zone B

Due to Groundwater Release

\begin{tabular}{rrrr}
\hline Time $(y r)$ & As & $\mathrm{Cr}$ & $\mathrm{Ni}$ \\
\hline 15 & $0.0 \mathrm{E}+00$ & $0.0 \mathrm{E}+00$ & $0.0 \mathrm{E}+00$ \\
20 & $0.0 \mathrm{E}+00$ & $0.0 \mathrm{E}+00$ & $0.0 \mathrm{E}+00$ \\
25 & $0.0 \mathrm{E}+00$ & $0.0 \mathrm{E}+00$ & $0.0 \mathrm{E}+00$ \\
30 & $0.0 \mathrm{E}+00$ & $0.0 \mathrm{E}+00$ & $0.0 \mathrm{E}+00$ \\
60 & $0.0 \mathrm{E}+00$ & $0.0 \mathrm{E}+00$ & $0.0 \mathrm{E}+00$ \\
62 & $0.0 \mathrm{E}+00$ & $0.0 \mathrm{E}+00$ & $0.0 \mathrm{E}+00$ \\
80 & $0.0 \mathrm{E}+00$ & $0.0 \mathrm{E}+00$ & $0.0 \mathrm{E}+00$ \\
100 & $0.0 \mathrm{E}+00$ & $0.0 \mathrm{E}+00$ & $0.0 \mathrm{E}+00$ \\
125 & $0.0 \mathrm{E}+00$ & $0.0 \mathrm{E}+00$ & $0.0 \mathrm{E}+00$ \\
575 & $0.0 \mathrm{E}+00$ & $0.0 \mathrm{E}+00$ & $0.0 \mathrm{E}+00$ \\
590 & $0.0 \mathrm{E}+00$ & $0.0 \mathrm{E}+00$ & $0.0 \mathrm{E}+00$ \\
620 & $0.0 \mathrm{E}+00$ & $0.0 \mathrm{E}+00$ & $0.0 \mathrm{E}+00$ \\
750 & $0.0 \mathrm{E}+00$ & $0.0 \mathrm{E}+00$ & $0.0 \mathrm{E}+00$ \\
1000 & $0.0 \mathrm{E}+00$ & $0.0 \mathrm{E}+00$ & $0.0 \mathrm{E}+00$ \\
1015 & $0.0 \mathrm{E}+00$ & $0.0 \mathrm{E}+00$ & $0.0 \mathrm{E}+00$ \\
1025 & $0.0 \mathrm{E}+00$ & $0.0 \mathrm{E}+00$ & $0.0 \mathrm{E}+00$ \\
1060 & $0.0 \mathrm{E}+00$ & $0.0 \mathrm{E}+00$ & $0.0 \mathrm{E}+00$ \\
1080 & $0.0 \mathrm{E}+00$ & $0.0 \mathrm{E}+00$ & $0.0 \mathrm{E}+00$ \\
1120 & $0.0 \mathrm{E}+00$ & $0.0 \mathrm{E}+00$ & $0.0 \mathrm{E}+00$ \\
1175 & $0.0 \mathrm{E}+00$ & $0.0 \mathrm{E}+00$ & $0.0 \mathrm{E}+00$ \\
1575 & $0.0 \mathrm{E}+00$ & $0.0 \mathrm{E}+00$ & $0.0 \mathrm{E}+00$ \\
1750 & $0.0 \mathrm{E}+00$ & $0.0 \mathrm{E}+00$ & $0.0 \mathrm{E}+00$ \\
2175 & $0.0 \mathrm{E}+00$ & $0.0 \mathrm{E}+00$ & $0.0 \mathrm{E}+00$ \\
4000 & $0.0 \mathrm{E}+00$ & $0.0 \mathrm{E}+00$ & $0.0 \mathrm{E}+00$ \\
6000 & $1.6 \mathrm{E}-02$ & $0.0 \mathrm{E}+00$ & $0.0 \mathrm{E}+00$ \\
8000 & $9.6 \mathrm{E}-02$ & $0.0 \mathrm{E}+00$ & $0.0 \mathrm{E}+00$ \\
10000 & $9.6 \mathrm{E}-02$ & $0.0 \mathrm{E}+00$ & $0.0 \mathrm{E}+00$ \\
& & & \\
\hline & & &
\end{tabular}

